\title{
Formation of dense partonic matter in relativistic nucleus-nucleus collisions at RHIC: Experimental evaluation by the PHENIX collaboration
}

\author{
K. Adcox ${ }^{\text {be S.S. Adler }}{ }^{\mathrm{e}}$ S. Afanasiev ${ }^{\mathrm{t}}$ C. Aidala ${ }^{\mathrm{e}, \mathrm{j}}$ \\ N.N. Ajitanand ${ }^{\text {aw }}$ Y. Akiba ${ }^{\text {w,aq,ar }}$ A. Al-Jamel ${ }^{\text {al }}$ J. Alexander aw \\ R. Amirikas ${ }^{n}$ K. Aoki ${ }^{\text {aa,aq }}$ L. Aphecetche ${ }^{\text {az }}$ Y. Arai ${ }^{w}$ \\ R. Armendariz ${ }^{a \ell}$ S.H. Aronson ${ }^{\mathrm{e}}$ R. Averbeck ${ }^{\text {ay }}$ T.C. Awes ${ }^{\mathrm{am}}$ \\ R. Azmoun ${ }^{e, a y}$ V. Babintsev ${ }^{q}$ A. Baldisseri ${ }^{k}$ K.N. Barish ${ }^{f}$ \\ P.D. Barnes ${ }^{\text {ad }}$ J. Barrette ${ }^{\text {ag }}$ B. Bassalleck ${ }^{\text {ak }}$ S. Bathe ${ }^{\text {f,ah }}$ \\ S. Batsouli ${ }^{j}$ V. Baublis ${ }^{\text {ap }}$ F. Bauer ${ }^{f}$ A. Bazilevsky ${ }^{e, q, a r}$ \\ S. Belikov ${ }^{\text {e,q,s }}$ F.G. Bellaiche ${ }^{\text {am }}$ S.T. Belyaev ${ }^{z}$ M.J. Bennett ${ }^{\text {ad }}$ \\ Y. Berdnikov at S. Bhagavatula ${ }^{\mathrm{s}}$ M.T. Bjorndal ${ }^{\mathrm{j}}$ \\ J.G. Boissevain ${ }^{\text {ad }}$ H. Borel ${ }^{k}$ S. Borenstein ${ }^{\text {ab }}$ S. Botelho ${ }^{\text {au }}$ \\ M.L. Brooks ${ }^{\text {ad }}$ D.S. Brown ${ }^{\text {al }}$ N. Bruner ${ }^{\text {ak }}$ D. Bucher ${ }^{\text {ah }}$ \\ H. Buesching e,ah V. Bumazhnov ${ }^{q}$ G. Bunce ${ }^{\mathrm{e}, \text { ar }}$ \\ J.M. Burward-Hoy ac,ad,ay S. Butsyk ${ }^{\text {ap,ay }}$ X. Camard ${ }^{\text {az }}$ \\ T.A. Carey ${ }^{\text {ad }}$ J.-S. Chai ${ }^{u}$ P. Chand ${ }^{\text {d }}$ J. Chang ${ }^{\text {f }}$ W.C. Chang ${ }^{b}$ \\ L.L. Chavez ${ }^{\text {ak }}$ S. Chernichenko ${ }^{q}$ C.Y. Chi ${ }^{j}$ J. Chiba $^{w}$ M. Chiu ${ }^{j}$ \\ I.J. Choi ${ }^{\text {bh }}$ J. Choi ${ }^{v}$ R.K. Choudhury ${ }^{d}$ T. Christ ${ }^{\text {ay }}$ \\ T. Chujo ${ }^{\text {e,bd,be M.S. Chung }}{ }^{\mathrm{y}, \text { ad }}$ P. Chung ${ }^{\text {aw }}$ V. Cianciolo ${ }^{\text {am }}$ \\ C.R. Cleven ${ }^{\circ}$ Y. Cobigo ${ }^{k}$ B.A. Cole ${ }^{j}$ M.P. Comets ${ }^{\text {an }}$ \\ P. Constantin ${ }^{\mathrm{s}}$ M. Csanád ${ }^{\mathrm{m}}$ T. Csörgö ${ }^{\mathrm{x}}$ J.P. Cussonneau ${ }^{\mathrm{az}}$ \\ D. d'Enterria ${ }^{j}$ T. Dahms ${ }^{\text {ay }}$ K. Das ${ }^{n}$ G. David ${ }^{\text {e F. Deák }}{ }^{\mathrm{m}}$ \\ H. Delagrange ${ }^{\text {az }}$ A. Denisov ${ }^{\mathrm{q}}$ A. Deshpande ${ }^{\text {ar,ay }}$ E.J. Desmond ${ }^{\mathrm{e}}$
} A. Devismes ${ }^{\text {ay }}$ O. Dietzsch ${ }^{\text {au }}$ B.V. Dinesh ${ }^{d}$ J.L. Drachenberg ${ }^{a}$ O. Drapier ${ }^{a b}$ A. Drees ${ }^{\text {ay }}$ A.K. Dubey ${ }^{\text {bg }}$ R. du Rietz ${ }^{\text {af }}$ A. Durum ${ }^{\text {q }}$ D. Dutta ${ }^{\text {d V. Dzhordzhadze }}{ }^{\text {ba }}$ K. Ebisu ${ }^{\text {aj }}$ Y.V. Efremenko am J. Egdemir ay K. El Chenawi be A. Enokizono ${ }^{\text {p H. En'yo }}{ }^{\text {aa,aq,ar }}$ B. Espagnon ${ }^{\text {an }}$ S. Esumi bd L. Ewell ${ }^{\text {e }}$ T. Ferdousi ${ }^{f}$ D.E. Fields ${ }^{\text {ak,ar }}$ C. Finck ${ }^{\text {az }}$ F. Fleuret ${ }^{\text {ab }}$ S.L. Fokin ${ }^{z}$ B. Forestier ${ }^{\text {ae }}$ B.D. Fox ${ }^{\text {ar }}$ Z. Fraenkel ${ }^{\text {bg }}$ 
J.E. Frantz ${ }^{\mathrm{j}}$ A. Franz ${ }^{\mathrm{e}}$ A.D. Frawley ${ }^{\mathrm{n}}$ Y. Fukao ${ }^{\text {aa,aq,ar }}$ S.-Y. Fung ${ }^{\mathrm{f}}$ S. Gadrat ${ }^{\text {ae }}$ S. Garpman ${ }^{\text {af }, 1}$ F. Gastineau ${ }^{\text {az }}$ M. Germain ${ }^{\text {az }}$ T.K. Ghosh ${ }^{\text {be }}$ A. Glenn ${ }^{\text {ba }}$ A.L. Godoi au G. Gogiberidze ${ }^{\text {ba }}$ M. Gonin ab J. Gosset ${ }^{\text {k }}$ Y. Goto aq,ar R. Granier de Cassagnac ${ }^{\text {ab }}$ N. Grau ${ }^{\mathrm{s}}$ S.V. Greene ${ }^{\text {be }}$ M. Grosse Perdekamp ${ }^{\text {r,ar }}$ T. Gunji ${ }^{\text {h }}$ S.K. Gupta ${ }^{d}$ W. Guryn ${ }^{\mathrm{e}}$ H.-A. Gustafsson ${ }^{\text {af }}$ T. Hachiya ${ }^{\text {paq }}$ A. Hadjhenni ${ }^{\text {az }}$ J.S. Haggerty ${ }^{\text {e M.N. Hagiwara }}{ }^{a}$ H. Hamagaki ${ }^{\text {h }}$ A.G. Hansen ${ }^{\text {ad }}$ H. Hara ${ }^{\text {aj }}$ H. Harada ${ }^{p}$ E.P. Hartouni ${ }^{\text {ac }}$ K. Haruna ${ }^{p}$ M. Harvey ${ }^{\mathrm{e}}$ E. Haslum ${ }^{\text {af }}$ K. Hasuko aq R. Hayano ${ }^{\text {h,bc }}$ N. Hayashi ${ }^{\text {aq }}$ X. He ${ }^{o}$ M. Heffner ${ }^{\text {ac }}$ T.K. Hemmick ${ }^{\text {ay }}$ J.M. Heuser ${ }^{\text {aq,ay }}$ M. Hibino ${ }^{\text {bf }}$ P. Hidas ${ }^{\mathrm{x}}$ H. Hiejima ${ }^{\mathrm{r}}$ J.C. Hills ${ }^{\mathrm{s}}$ D.S. Ho ${ }^{\text {bh }}$ R. Hobbs ${ }^{\text {ak }}$ M. Holmes be W. Holzmann ${ }^{\text {aw }}$ K. Homma ${ }^{\mathrm{p}}$ B. Hong ${ }^{\mathrm{y}}$ A. Hoover ${ }^{a \ell}$ T. Horaguchi aq,ar,bb H.M. Hur ${ }^{\text {u }}$ T. Ichihara ${ }^{\text {aq,ar }}$ V.V. Ikonnikov ${ }^{z}$ K. Imai aa,aq M. Inaba bd M. Inuzuka ${ }^{\text {h }}$ M.S. Ippolitov ${ }^{z} D$. Isenhower ${ }^{a} L$. Isenhower ${ }^{a} M$ M. Ishihara aq,ar T. Isobe ${ }^{\text {h }}$ M. Issah ${ }^{\text {aw }}$ A. Isupov ${ }^{t}$ B.V. Jacak ${ }^{\text {ar,ay }}$ W.Y. Jang ${ }^{y}$ Y. Jeong ${ }^{\mathrm{v}}$ J. Jia $^{\mathrm{j}, \mathrm{ay}}$ J. Jin $^{\mathrm{j}}$ O. Jinnouchi ${ }^{\mathrm{aq}, \mathrm{ar}}$ B.M. Johnson ${ }^{\mathrm{e}}$ S.C. Johnson ac,ay K.S. Joo ai D. Jouan ${ }^{\text {an }}$ F. Kajihara ${ }^{\text {h,aq }}$ S. Kametani ${ }^{\text {h,bf }}$ N. Kamihara ${ }^{\text {aq,bb }}$ M. Kaneta ${ }^{\text {ar }}$ J.H. Kang ${ }^{\text {bh }}$ M. Kann ${ }^{\text {ap }}$ S.S. Kapoor ${ }^{d}$ K. Katou ${ }^{\text {bf }}$ T. Kawabata ${ }^{\text {h }}$

T. Kawagishi ${ }^{\text {bd }}$ A.V. Kazantsev ${ }^{z}$ S. Kelly ${ }^{i, j}$ B. Khachaturov ${ }^{\text {bg }}$ A. Khanzadeev ap J. Kikuchi ${ }^{\text {bf }}$ D.H. Kim ${ }^{\text {ai }}$ D.J. Kim ${ }^{\text {bh }}$ D.W. Kim ${ }^{v}$ E. Kim ${ }^{\text {av }}$ G.-B. Kim ${ }^{\text {ab }}$ H.J. Kim ${ }^{\text {bh }}$ S.Y. Kim ${ }^{\text {bh }}$ Y.-S. Kim ${ }^{\text {u }}$ Y.G. Kim ${ }^{\text {bh }}$ E. Kinney ${ }^{\mathrm{i}}$ W.W. Kinnison ${ }^{\text {ad }}$ A. Kiss ${ }^{\mathrm{m}}$ E. Kistenev ${ }^{\mathrm{e}}$ A. Kiyomichi ${ }^{\text {aq,bd }}$ K. Kiyoyama ${ }^{\text {aj }}$ C. Klein-Boesing ah S. Klinksiek ${ }^{\text {ak }}$ H. Kobayashi ${ }^{\text {aq,ar }}$ L. Kochenda ${ }^{\text {ap }}$ V. Kochetkov ${ }^{q}$ D. Koehler ${ }^{\text {ak }}$ T. Kohama ${ }^{p}$ R. Kohara ${ }^{\mathrm{p}}$ B. Komkov ap M. Konno ${ }^{\text {bd }}$ M. Kopytine ${ }^{\text {ay }}$

D. Kotchetkov ${ }^{f}$ A. Kozlov ${ }^{\text {bg }}$ P.J. Kroon ${ }^{e}$ C.H. Kuberg ${ }^{\text {a,ad }}$ G.J. Kunde ${ }^{\text {ad }}$ N. Kurihara ${ }^{h}$ K. Kurita aq,ar,as $Y$. Kuroki bd M.J. Kweon ${ }^{y}$ Y. Kwon ${ }^{\text {bh }}$ G.S. Kyle ${ }^{\text {a }}$ R. Lacey ${ }^{\text {aw }}$ V. Ladygin ${ }^{\mathrm{t}}$ J.G. Lajoie ${ }^{\mathrm{s}}$ J. Lauret ${ }^{\mathrm{aw}}$ Y. Le Bornec ${ }^{\text {an }}$ A. Lebedev ${ }^{\mathrm{s}, \mathrm{z}}$ S. Leckey ${ }^{\text {ay }}$ D.M. Lee ${ }^{\text {ad }}$ M.K. Lee ${ }^{\text {bh }}$ S. Lee ${ }^{\text {v }}$ M.J. Leitch ${ }^{\text {ad }}$ M.A.L. Leite ${ }^{\text {au }}$ X.H. $\operatorname{Li}^{f}$ Z. Li ${ }^{\text {gaq }}$ D.J. Lim ${ }^{\text {bh }}$ H. Lim ${ }^{\text {av }}$ 
A. Litvinenko ${ }^{t}$ M.X. Liu ${ }^{\text {ad }}$ X. Liu $^{g}{ }^{g}$ Y. Liu ${ }^{\text {an }}$ Z. Liu ${ }^{g}$ C.F. Maguire ${ }^{\text {be }}$ J. Mahon ${ }^{\text {e }}$ Y.I. Makdisi ${ }^{\mathrm{e}}$ A. Malakhov ${ }^{\mathrm{t}}$ M.D. Malik ${ }^{\text {ak }}$ V.I. Manko ${ }^{\text {Z }}$ Y. Mao ${ }^{\text {g,ao,aq }}$ S.K. Mark ${ }^{\text {ag }}$ S. Markacs ${ }^{j}$ G. Martinez ${ }^{\text {az }}$ M.D. Marx ${ }^{\text {ay }}$ A. Masaike ${ }^{\text {aa }}$ H. Masui ${ }^{\text {bd }}$ F. Matathias ay T. Matsumoto ${ }^{\text {h,bf }}$ M.C. McCain ${ }^{\text {a,r }}$

P.L. McGaughey ${ }^{\text {ad }}$ E. Melnikov ${ }^{\mathrm{q}}$ M. Merschmeyer ${ }^{\text {ah }}$ F. Messer ${ }^{\text {ay }}$ M. Messer ${ }^{\text {e }}$ Y. Miake ${ }^{\text {bd }}$ J. Milan ${ }^{\text {aw }}$ T.E. Miller ${ }^{\text {be }}$ A. Milov ay,bg S. Mioduszewski ${ }^{\text {eba }}{ }^{\text {R. E. Mischke }}{ }^{\text {ad }}$ G.C. Mishra ${ }^{\circ}$ J.T. Mitchell ${ }^{\text {e A.K. Mohanty }}{ }^{\text {d D.P. Morrison }}{ }^{\text {e J.M. Moss }}{ }^{\text {ad }}$ T.V. Moukhanova ${ }^{z}$ F. Mühlbacher ay D. Mukhopadhyay be,bg M. Muniruzzaman ${ }^{\text {f }}$ J. Murata ${ }^{\text {aq,ar,as }}$ S. Nagamiya ${ }^{\text {w }}$ Y. Nagasaka ${ }^{\text {aj }}$ Y. Nagata ${ }^{\text {bd }}$ J.L. Nagle ${ }^{\mathrm{i}, \mathrm{j}}$ M. Naglis ${ }^{\text {bg }}$ Y. Nakada ${ }^{\text {aa }}$ T. Nakamura ${ }^{p}$ B.K. Nandi ${ }^{f}$ M. Nara ${ }^{\text {bd }}$ J. Newby ${ }^{\text {ac,ba }}$ M. Nguyen ${ }^{\text {ay }}$ L. Nikkinen ${ }^{\text {ag }}$ P. Nilsson ${ }^{\text {af }}$ S. Nishimura ${ }^{\text {h }}$ B. Norman ${ }^{\text {ad }}$ A.S. Nyanin ${ }^{z}$ J. Nystrand ${ }^{\text {af }}$ E. O'Brien ${ }^{\text {e C.A. Ogilvie }}{ }^{\mathrm{s}}$ H. Ohnishi ${ }^{\mathrm{e}, \mathrm{p}, \mathrm{aq}}$ I.D. Ojha ${ }^{\text {c,be }}$ H. Okada ${ }^{\text {aa,aq }}$ K. Okada ${ }^{\text {aq,ar }}$ O.O. Omiwade ${ }^{\text {a }}$ M. Ono ${ }^{\text {bd }}$ V. Onuchin ${ }^{\text {q }}$ A. Oskarsson ${ }^{\text {af }}$ L. Österman ${ }^{\text {af }}$ I. Otterlund ${ }^{\text {af }}$ K. Oyama ${ }^{\text {h,bc }}$ K. Ozawa ${ }^{h}$ L. Paffrath ${ }^{e, 1}$ D. Pal be,bg A.P.T. Palounek ${ }^{\text {ad }}$ V. Pantuev ay V. Papavassiliou ${ }^{\text {al }}$ J. Park $^{\text {av }}$ W.J. Park ${ }^{\mathrm{y}}$ A. Parmar ${ }^{\text {ak }}$ S.F. Pate ${ }^{\text {a }}$ H. Pei ${ }^{\mathrm{s}}$ T. Peitzmann ${ }^{\text {ah }}$ V. Penev ${ }^{t}$ J.-C. Peng ${ }^{\text {r,ad }}$ H. Pereira ${ }^{k}$ V. Peresedov ${ }^{t}$ D.Yu. Peressounko ${ }^{z}$ A.N. Petridis ${ }^{\mathrm{s}}$ A. Pierson ${ }^{\text {ak }}$ C. Pinkenburg e,aw R.P. Pisani ${ }^{\text {e }}$ P. Pitukhin ${ }^{\mathrm{q}}$ F. Plasil ${ }^{\text {am }}$ M. Pollack ${ }^{\text {ay,ba }}$ K. Pope ${ }^{\text {ba }}$ M.L. Purschke ${ }^{\text {e }}$ A.K. Purwar ay H. $\mathrm{Qu}^{\mathrm{O}}$ J.M. Qualls ${ }^{\mathrm{a}}{ }^{\text {J. Rak }}{ }^{\mathrm{s}}$ I. Ravinovich ${ }^{\text {bg }}$ K.F. Read ${ }^{\mathrm{am}, \mathrm{ba}}$ M. Reuter ${ }^{\text {ay }}$ K. Reygers ${ }^{\text {ah }}$ V. Riabov ${ }^{\text {ap,at }}$ Y. Riabov ${ }^{\text {ap }}$

G. Roche ${ }^{\text {ae }}$ A. Romana ${ }^{\text {ab }}$ M. Rosati ${ }^{\text {s }}$ A.A. Rose ${ }^{\text {be }}$ S.S.E. Rosendahl ${ }^{\text {af }}$ P. Rosnet ${ }^{\text {ae }}$ P. Rukoyatkin ${ }^{\text {t }}$ V.L. Rykov ${ }^{\text {aq }}$ S.S. Ryu ${ }^{\text {bh }}$ M.E. Sadler ${ }^{\text {a }}$ B. Sahlmueller ah N. Saito aa,aq,ar A. Sakaguchi ${ }^{\text {p T. Sakaguchi }}{ }^{\text {h,bf }}$ M. Sakai ${ }^{\text {aj }}$ S. Sakai ${ }^{\text {bd }}$ H. Sako bd T. Sakuma aq,bb V. Samsonov ap L. Sanfratello ak T.C. Sangster ${ }^{\text {ac }}$ R. Santo ${ }^{\text {ah }}$ H.D. Sato ${ }^{\text {aa,aq }}$ S. Sato ${ }^{\text {e,w,bd }}$ S. Sawada ${ }^{\text {w }}$ B.R. Schlei ${ }^{\text {ad }}$ Y. Schutz ${ }^{\text {az }}$ V. Semenov ${ }^{\text {q }}$ R. Seto ${ }^{f}$ D. Sharma ${ }^{\text {bg }}$ M.R. Shaw ${ }^{\text {a,ad }}$ T.K. Shea ${ }^{\text {e I. Shein }}{ }^{q}$ 
T.-A. Shibata ${ }^{\text {aq,bb }}$ K. Shigaki ${ }^{\text {p,w }}$ T. Shiina ${ }^{\text {ad }}$ M. Shimomura ${ }^{\text {bd }}$ Y.H. Shin ${ }^{\text {bh }}$ T. Shohjoh ${ }^{\text {bd }}$ K. Shoji ${ }^{\text {aa,aq }}$ I.G. Sibiriak ${ }^{z}$ A. Sickles ${ }^{\text {ay }}$ C.L. Silva ${ }^{\text {au }}$ D. Silvermyr ${ }^{\text {ad,af,am K.S. Sim }}{ }^{\mathrm{y}}$ J. Simon-Gillo ad C.P. Singh ${ }^{\text {c }}$ V. Singh ${ }^{c}$ M. Sivertz ${ }^{\text {e }}$ S. Skutnik ${ }^{\mathrm{s}}$ W.C. Smith ${ }^{\text {a }}$ A. Soldatov ${ }^{\text {q R.A. Soltz }}{ }^{\text {ac }}$ W.E. Sondheim ${ }^{\text {ad }}$ S.P. Sorensen ${ }^{\text {am,ba }}$ I.V. Sourikova ${ }^{e}$ F. Staley ${ }^{k}$ P.W. Stankus am N. Starinsky ${ }^{\text {ag }}$ P. Steinberg ${ }^{j}$ E. Stenlund ${ }^{\text {af }}$ M. Stepanov ${ }^{\text {a }}$ A. Ster ${ }^{x}$ S.P. Stoll ${ }^{\text {e M. Sugioka }}{ }^{\text {aq,bb }}$ T. Sugitate ${ }^{p}$ C. Suire ${ }^{\text {an }}$ J.P. Sullivan ${ }^{\text {ad }}$ Y. Sumi ${ }^{\text {P Z. Sun }}{ }^{\mathrm{g}}$ M. Suzuki ${ }^{\text {bd }}$ J. Sziklai ${ }^{\mathrm{x}}$ T. Tabaru ${ }^{\text {ar }}$ S. Takagi bd E.M. Takagui au A. Taketani aq,ar M. Tamai ${ }^{\text {bf }}$ K.H. Tanaka ${ }^{w}$ Y. Tanaka ${ }^{\text {aj }}$ K. Tanida ${ }^{\text {aq,ar }}$ E. Taniguchi ${ }^{\text {aq,bb }}$ M.J. Tannenbaum ${ }^{\mathrm{e}}$ A. Taranenko ${ }^{\text {aw }}$ P. Tarján ${ }^{\ell}$ J.D. Tepe ${ }^{\text {a,ad }}$ J. Thomas ${ }^{\text {ay }}$ J.H. Thomas ${ }^{\text {ac }}$ T.L. Thomas ${ }^{\text {ak }}$ W. Tian ${ }^{\text {g,ba }}$ M. Togawa ${ }^{\text {aa,aq }}$ J. Tojo aa,aq H. Torii ${ }^{\text {aa,aq,ar }}$ R.S. Towell ${ }^{\text {a,ad }}$ V-N. Tram ${ }^{\text {ab }}$ I. Tserruya ${ }^{b g}$ Y. Tsuchimoto ${ }^{\text {p,aq }}$ H. Tsuruoka ${ }^{\text {bd }}$ A.A. Tsvetkov ${ }^{\text {z }}$ S.K. Tuli ${ }^{\mathrm{c}}$ H. Tydesjo ${ }^{\text {af }}$ N. Tyurin ${ }^{\text {q T.J. Uam }}{ }^{\text {ai }}$ T. Ushiroda ${ }^{\text {aj }}$ H. Valle be H.W. van Hecke ${ }^{\text {ad }}$ C. Velissaris ${ }^{\text {al }}$ J. Velkovska ${ }^{\text {e,ay, be }}$ M. Velkovsky ay R. Vertesi ${ }^{\ell}$ V. Veszprémi ${ }^{\ell}$ L. Villatte ${ }^{\text {ba }}$ A.A. Vinogradov ${ }^{z}$ M.A. Volkov ${ }^{z}$ A. Vorobyov ${ }^{\text {ap }}$ E. Vznuzdaev ap M. Wagner ${ }^{\text {aa }}$ H. Wang ${ }^{f}$ X.R. Wang ${ }^{\text {o,al }}$ Y. Watanabe ${ }^{\text {aq,ar }}$ J. Wessels ${ }^{\text {ah }}$ S.N. White ${ }^{\mathrm{e}} \mathrm{N}$. Willis ${ }^{\text {an }}$ D. Winter ${ }^{\mathrm{j}}$ C. Witzig ${ }^{\mathrm{e}}$ F.K. Wohn ${ }^{\mathrm{s}}$ C.L. Woody ${ }^{\mathrm{e}}$ M. Wysocki ${ }^{\mathrm{i}}$ W. Xie ${ }^{\text {f,ar,bo }}$ K. Yagi bd Y. Yang ${ }^{\mathrm{g}}$ A. Yanovich ${ }^{\mathrm{q}}$ S. Yokkaichi aq,ar G.R. Young am I. Younus ${ }^{\text {ak }}$ I.E. Yushmanov ${ }^{z}$ W.A. Zajc ${ }^{j}$ O. Zaudkte ${ }^{\text {ah }}$ C. Zhang ${ }^{j}$ Z. Zhang ${ }^{\text {ay }}$ S. Zhou ${ }^{\text {g }}$ S.J. Zhou ${ }^{\text {bg }}$ J. Zimányi ${ }^{\mathrm{x}}$ L. Zolin ${ }^{\mathrm{t}} \mathrm{X}$. Zong ${ }^{\mathrm{s}}$ (PHENIX Collaboration)

a Abilene Christian University, Abilene, TX 79699, USA

b Institute of Physics, Academia Sinica, Taipei 11529, Taiwan

${ }^{\mathrm{c}}$ Department of Physics, Banaras Hindu University, Varanasi 221005, India ${ }^{\mathrm{d}}$ Bhabha Atomic Research Centre, Bombay 400 085, India

e Brookhaven National Laboratory, Upton, NY 11973-5000, USA

${ }^{\mathrm{f}}$ University of California - Riverside, Riverside, CA 92521, USA

${ }^{g}$ China Institute of Atomic Energy (CIAE), Beijing, People's Republic of China

${ }^{\mathrm{h}}$ Center for Nuclear Study, Graduate School of Science, University of Tokyo, 7-3-1 
Hongo, Bunkyo, Tokyo 113-0033, Japan

${ }^{\mathrm{i}}$ University of Colorado, Boulder, CO 80309

${ }^{\mathrm{j} C o l u m b i a}$ University, New York, NY 10027 and Nevis Laboratories, Irvington, NY 10533, USA

k Dapnia, CEA Saclay, F-91191, Gif-sur-Yvette, France

${ }^{\ell}$ Debrecen University, H-4010 Debrecen, Egyetem tér 1, Hungary

${ }^{\mathrm{m}}$ ELTE, Eötvös Loránd University, H - 1117 Budapest, Pázmány P. s. 1/A,

Hungary

${ }^{\mathrm{n}}$ Florida State University, Tallahassee, FL 32306, USA

${ }^{\circ}$ Georgia State University, Atlanta, GA 30303, USA

p Hiroshima University, Kagamiyama, Higashi-Hiroshima 739-8526, Japan

${ }^{\mathrm{q}}$ Institute for High Energy Physics (IHEP), Protvino, Russia

${ }^{\mathrm{r}}$ University of Illinois at Urbana-Champaign, Urbana, IL 61801

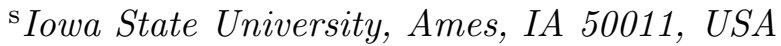

${ }^{\mathrm{t}}$ Joint Institute for Nuclear Research, 141980 Dubna, Moscow Region, Russia

" KAERI, Cyclotron Application Laboratory, Seoul, South Korea

${ }^{\vee}$ Kangnung National University, Kangnung 210-702, South Korea

${ }^{\mathrm{w}}$ KEK, High Energy Accelerator Research Organization, Tsukuba-shi, Ibaraki-ken 305-0801, Japan

${ }^{\mathrm{x}}$ KFKI Research Institute for Particle and Nuclear Physics (RMKI), H-1525 Budapest 114, POBox 49, Hungary

${ }^{\text {y } K o r e a ~ U n i v e r s i t y, ~ S e o u l, ~ 136-701, ~ K o r e a ~}$

${ }^{\mathrm{z}}$ Russian Research Center "Kurchatov Institute", Moscow, Russia

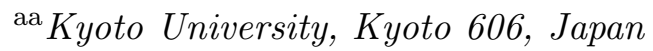

${ }^{\mathrm{ab}}$ Laboratoire Leprince-Ringuet, Ecole Polytechnique, CNRS-IN2P3, Route de Saclay, F-91128, Palaiseau, France

${ }^{\mathrm{ac}}$ Lawrence Livermore National Laboratory, Livermore, CA 94550, USA

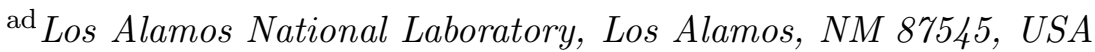

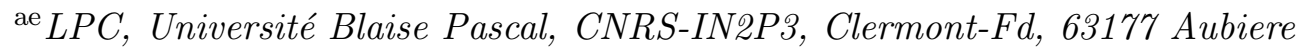
Cedex, France

af Department of Physics, Lund University, Box 118, SE-221 00 Lund, Sweden ag McGill University, Montreal, Quebec H3A 2T8, Canada

${ }^{\text {ah }}$ Institut für Kernphysik, University of Muenster, D-48149 Muenster, Germany

ai Myongji University, Yongin, Kyonggido 449-728, Korea

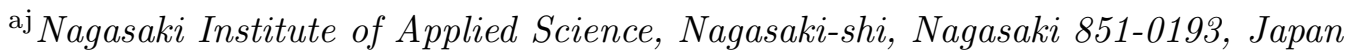

${ }^{\text {ak }}$ University of New Mexico, Albuquerque, NM 87131, USA

${ }^{\mathrm{a} \ell}$ New Mexico State University, Las Cruces, NM 88003, USA

am Oak Ridge National Laboratory, Oak Ridge, TN 37831, USA 
an IPN-Orsay, Universite Paris Sud, CNRS-IN2P3, BP1, F-91406, Orsay, France

ao Peking University, Beijing, People's Republic of China

ap PNPI, Petersburg Nuclear Physics Institute, Gatchina, Russia

${ }^{\text {aq }}$ RIKEN (The Institute of Physical and Chemical Research), Wako, Saitama 351-0198, JAPAN

${ }^{\text {ar } R I K E N ~ B N L ~ R e s e a r c h ~ C e n t e r, ~ B r o o k h a v e n ~ N a t i o n a l ~ L a b o r a t o r y, ~ U p t o n, ~ N Y ~}$ 11973-5000, USA

as Physics Department, Rikkyo University, 3-34-1 Nishi-Ikebukuro, Toshima, Tokyo 171-8501, Japan

${ }^{a t}$ St. Petersburg State Technical University, St. Petersburg, Russia

${ }^{a u}$ Universidade de São Paulo, Instituto de Física, Caixa Postal 66318, São Paulo CEP05315-970, Brazil

${ }^{a v}$ System Electronics Laboratory, Seoul National University, Seoul, South Korea

${ }^{\text {aw }}$ Chemistry Department, Stony Brook University, Stony Brook, SUNY, NY 11794-3400, USA

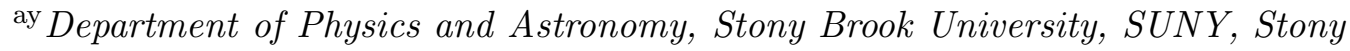
Brook, NY 11794, USA

${ }^{\mathrm{az}}$ SUBATECH (Ecole des Mines de Nantes, CNRS-IN2P3, Université de Nantes) BP 20722 - 44307, Nantes, France

ba University of Tennessee, Knoxville, TN 37996, USA

${ }^{\mathrm{bb}}$ Department of Physics, Tokyo Institute of Technology, Tokyo, 152-8551, Japan

bc University of Tokyo, Tokyo, Japan

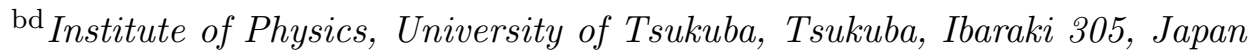

be Vanderbilt University, Nashville, TN 37235, USA

${ }^{\text {bf } W a s e d a}$ University, Advanced Research Institute for Science and Engineering, 17 Kikui-cho, Shinjuku-ku, Tokyo 162-0044, Japan

bg Weizmann Institute, Rehovot 76100, Israel

${ }^{\text {bh }}$ Yonsei University, IPAP, Seoul 120-749, Korea

\begin{abstract}
Extensive experimental data from high-energy nucleus-nucleus collisions were recorded using the PHENIX detector at the Relativistic Heavy Ion Collider (RHIC). The comprehensive set of measurements from the first three years of RHIC operation includes charged particle multiplicities, transverse energy, yield ratios and spectra of identified hadrons in a wide range of transverse momenta $\left(p_{T}\right)$, elliptic flow, two-particle correlations, non-statistical fluctuations, and suppression of particle production at high $p_{T}$. The results are examined with an emphasis on implications for the formation of a new state of dense matter. We find that the state of matter created at RHIC cannot be described in terms of ordinary color neutral hadrons.
\end{abstract}




\section{INTRODUCTION}

\subsection{Historical Introduction}

A recurring theme in the history of physics is the desire to study matter under extreme conditions. The latter half of the twentieth century saw this quest extended from 'ordinary' atomic systems to those composed of nuclear matter. Even prior to the identification of Quantum Chromodynamics (QCD) as the underlying theory of the strong interaction, there was considerable interest in the fate of nuclear matter when subjected to density and temperature extremes $[1,2,3]$. Particularly intriguing was the suggestion that new phases of nuclear matter could be associated with a corresponding change in the structure of the vacuum [4]. These considerations gained additional impetus with the realizations that a) QCD was the correct theory of the strong interaction, b) the phenomena of quark confinement was a consequence of the nonperturbative structure of the vacuum and c) this vacuum structure is modified at high temperatures and/or densities, suggesting that quarks and gluons under such conditions would be deconfined. Taken together, these facts suggest that QCD is a fundamental theory of nature containing a phase transition that is accessible to experimental investigation.

It is quite remarkable that this understanding was achieved very early in the development of QCD. Collins and Perry noted in 1975 [5] that the reduction of the coupling constant at small distances indicated that the dense nuclear matter at the center of neutron stars would consist of deconfined quarks and gluons ${ }^{2}$. Their treatment focused on the high-density, low-temperature regime of QCD, but they did note that similar arguments might apply to the high temperatures present in the early universe. An extensive review by Shuryak in 1980 [7] is the first to have examined the high-temperature phase in detail, and is also notable for proposing the phrase "quark-gluon plasma" (QGP) to describe the deconfined state:

When the energy density $\varepsilon$ exceeds some typical hadronic value ( $\sim$ GeV/fm $\left.{ }^{3}\right)$, matter no longer consists of separate hadrons (protons, neutrons, etc.), but

\footnotetext{
Email address: PHENIXSpokesperson: zajc@nevis.columbia.edu (W.A. Zajc).

1 Deceased

2 In fact, prior to the development of QCD the quark hypothesis raised serious issues concerning the stability of neutron stars [6].
} 
as their fundamental constituents, quarks and gluons. Because of the apparent analogy with similar phenomena in atomic physics we may call this phase of matter the QCD (or quark-gluon) plasma.

Developing a quantitative understanding of the deconfining phase transition in hadronic matter and of QGP properties has proven to be a challenging task. While simple dimensional arguments suffice to identify both the critical energy density $\varepsilon_{C} \sim 1 \mathrm{GeV} / \mathrm{fm}^{3}$ and the associated critical temperature $T_{C} \sim 170$ $\mathrm{MeV}$, these values also imply that the transition occurs in a regime where the coupling constant is of order unity, thereby making perturbative descriptions highly suspect.

Progress in understanding QCD in the extremely non-perturbative domain near the critical temperature has relied on an essential contribution by Creutz [8], who showed that numerical implementations of Wilson's lattice formulation [9] could be used to study phase transition phenomena. This work, together with the continued exponential increases in computing power, stimulated the development of lattice QCD, which in turn has led to detailed investigations of the thermodynamic properties of quarks and gluons [10].

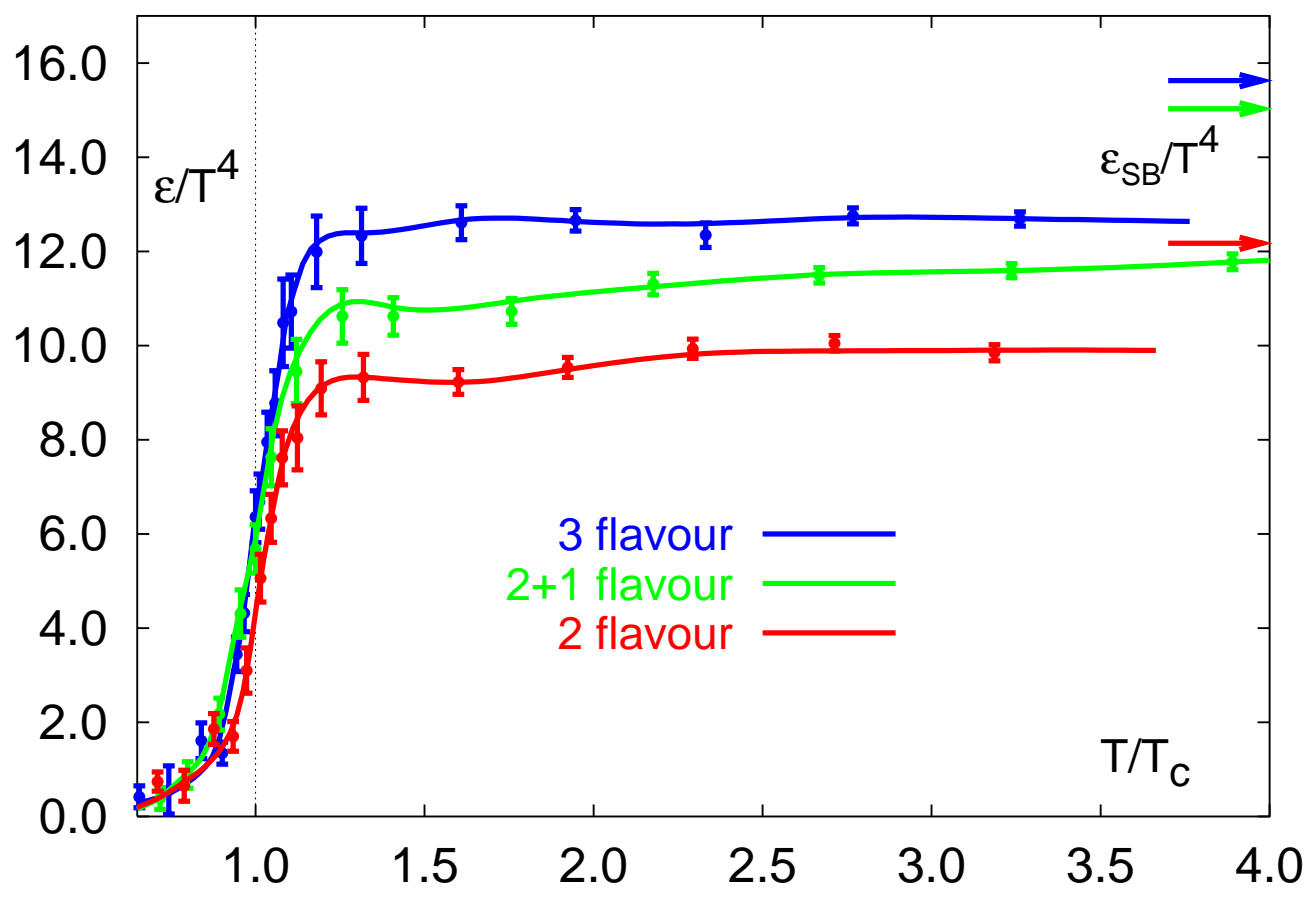

Fig. 1. Lattice QCD results [11] for the energy density / $T^{4}$ as a function of the temperature scaled by the critical temperature $T_{C}$. Note the arrows on the right side indicating the values for the Stefan-Boltzmann limit.

Lattice QCD predicts a phase transformation to a quark-gluon plasma at a temperature of approximately $T \approx 170 \mathrm{MeV} \approx 10^{12} \mathrm{~K}$, as shown in Fig. 1 [11]. 
This transition temperature corresponds to an energy density $\varepsilon \approx 1 \mathrm{GeV} / \mathrm{fm}^{3}$, nearly an order of magnitude larger than that of normal nuclear matter. As noted above, this value is plausible based on dimensional grounds, since such densities correspond to the total overlap of several (light) hadrons within a typical hadron volume of $1-3 \mathrm{fm}^{3}$. No plausible mechanism exists under which hadrons could retain their in vacuo properties under these conditions. Lattice calculations also indicate that this significant change in the behavior of the system occurs over a small range in temperature $(\sim 20 \mathrm{MeV})$, and suggest that the change of phase includes the restoration of approximate chiral symmetry resulting from greatly reduced or vanishing quark constituent masses.

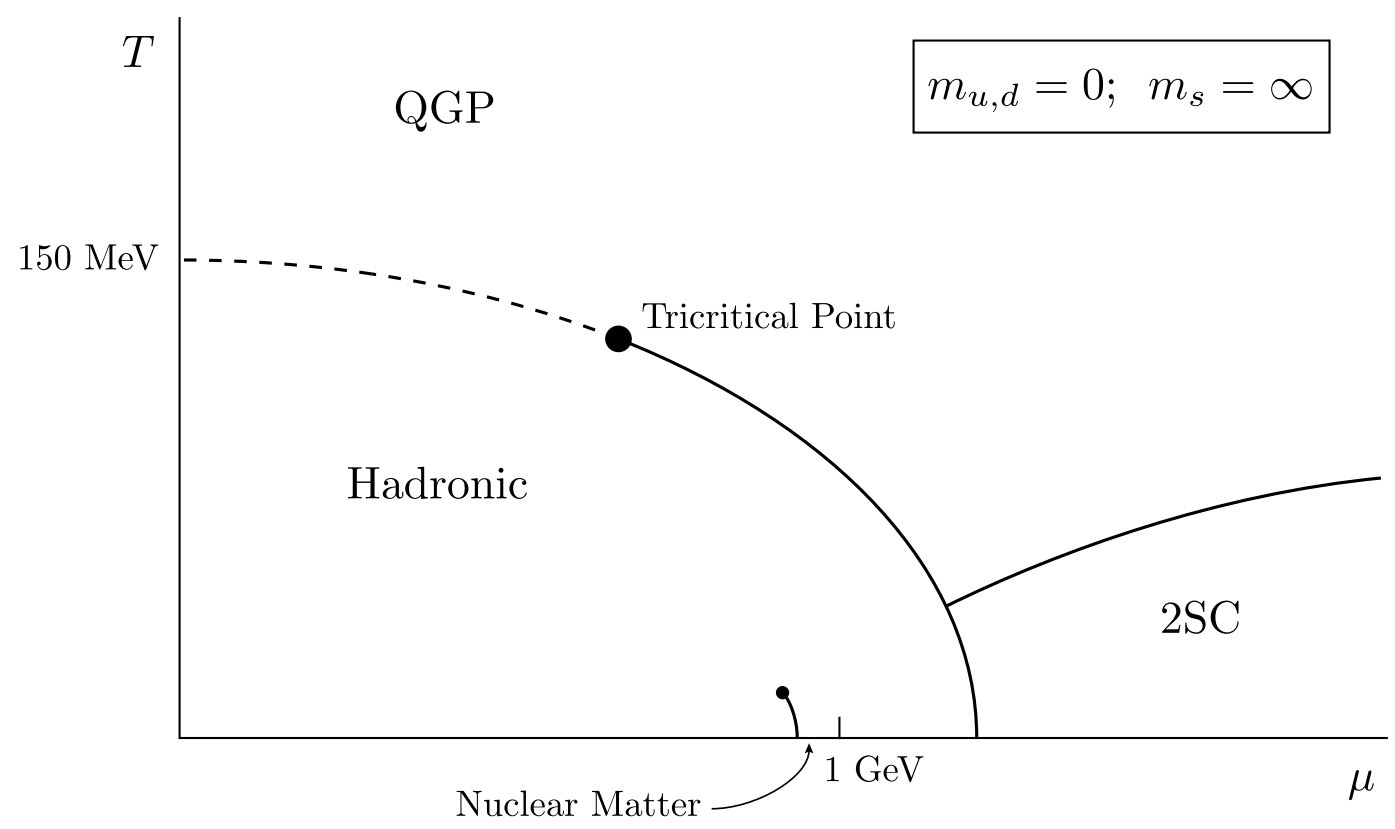

Fig. 2. Theoretical phase diagram of nuclear matter for two massless quarks as a function of temperature $T$ and baryon chemical potential $\mu$ [12].

In the limit of massless noninteracting particles, each bosonic degree of freedom contributes $\frac{\pi^{2}}{30} T^{4}$ to the energy density; each fermionic degree of freedom contributes $\frac{7}{8}$ this value. The corresponding "Stefan-Boltzmann" limits of the energy density $\varepsilon_{S B}$ for the case of 2(3) active flavor quark-gluon plasma is then

$$
\varepsilon_{S B}=\begin{aligned}
& \left\{2_{f} \cdot 2_{s} \cdot 2_{q} \cdot 3 \frac{7}{8}+2_{s} \cdot 8_{c}\right\} \frac{\pi^{2}}{30} T^{4}=37 \frac{\pi^{2}}{30} T^{4} \\
& \left\{3_{f} \cdot 2_{s} \cdot 2_{q} \cdot 3_{c} \frac{7}{8}+2_{s} \cdot 8_{c}\right\} \frac{\pi^{2}}{30} T^{4}=47.5 \frac{\pi^{2}}{30} T^{4}
\end{aligned}
$$

after summing over the appropriate flavor, spin, quark/antiquark and color factors for quarks and spin times color factors for gluons. The large numerical 
coefficients (37 and 47.5) stand in stark contrast to the value of $\sim 3$ expected for a hadron gas with temperature $T<T_{C}$, in which case the degrees of freedom are dominated by the three pion species $\pi^{-}, \pi^{0}, \pi^{+}$.

The exact order of this phase transition is not known. In a pure gauge theory containing only gluons the transition appears to be first order. However, inclusion of two light quarks (up and down) or three light quarks (adding the strange quark) can change the transition from first order to second order to a smooth crossover. These results are obtained at zero net baryon density; dramatic changes in the nature of the transition and in the medium itself are expected when the net baryon density becomes significant. A schematic version of the phase diagram for an idealized form of nuclear matter with vanishing light quark (up and down) masses and infinite strange quark mass is presented in Fig. 2 [12]. For sufficiently large values of the baryon chemical potential $\mu$ this system exhibits a first order phase transition between hadronic matter and QGP, along with a tricritical point below which the transition becomes second order. However, non-zero values of the light quark masses dramatically alter this simple picture: The second order phase transition denoted by the dashed line in Fig. 2 becomes a smooth crossover, and the tricritical point correspondingly becomes a critical point designating the end of the first order transition found at higher values of $\mu$. For example, recent calculations $[13,14]$ indicate that the transition is a crossover for values of $\mu<\sim 400 \mathrm{MeV}$. Given that both theoretical arguments and experimental data suggest that nucleusnucleus collisions at RHIC (at least near mid-rapidity) are characterized by low net baryon density, we will restrict our attention to this regime, while noting that the predicted smooth nature of the transition in this region increases the experimental challenges of unambiguously establishing that such a transition has occurred. We also note that while Fig. 2 shows that the region of low temperature and high baryon density is expected to show a transition to a color superconducting phase of matter, this regime is not accessible to RHIC collisions and will not be discussed further.

While the lattice results plotted in Fig. 1 show that the energy density reaches a significant fraction $(\sim 0.8)$ of the Stefan-Boltzmann values in the deconfined phase, the deviation from $\varepsilon_{S B}$, and the reason for the persistence of that deviation to the highest studied values of $T / T_{C}$, are of great interest. For instance, Greiner has noted [15] that "in order to allow for simple calculations the QGP is usually described as a free gas consisting of quarks and gluons. This is theoretically not well founded at $T \approx T_{c}$ ". In fact, analysis of the gluon propagator in a thermal system [16,17] has demonstrated that effective masses of order $g(T) T$ are generated, suggesting that the relevant degrees of freedom are in fact massive near $T_{C} \cdot m_{g} \approx T_{c}$ could be generated by gluons. Especially interesting is recent work which indicates that both heavy $[18,19,20]$ and light [21] flavor states may remain bound above $T_{C}$, calling into question the naive interpretation of $\varepsilon(T)$ as an indicator of the explicit appearance of 
quark and gluon degrees of freedom. This is supported by explicit calculations of the spectrum of bound states above $T_{C}$ [22] which predict a rich structure of states that belies a description as a weakly interacting parton gas.

To emphasize this point, consider the standard measure of the degree of coupling in a classical plasma, obtained by comparing the relative magnitudes of the average kinetic and potential energies:

$$
\Gamma \equiv \frac{\langle V(r)\rangle}{\left\langle E_{k i n}\right\rangle} .
$$

In the case of the QCD plasma the mean inter-particle spacing should scale as some numerical coefficient times $1 / T$. Naively, this gives a mean potential energy $\langle V(r)\rangle \sim \alpha_{s}(T)\langle 1 / r\rangle \sim \alpha_{s}(T) T$, leading to

$$
\Gamma \sim \frac{\alpha_{s}(T) T}{3 T} \sim \alpha_{s}(T) .
$$

Any reasonable estimate for the numerical coefficients leads to $\Gamma>1$, which is the condition for a "strongly-coupled" plasma. In reality, the screening present at such densities (or equivalently, the generation of effective gluon masses) modifies the mean potential energy to $\langle V(r)\rangle \sim g(T) T$, which only increases the estimated value of $\Gamma$ [23]. Considerations such as these have led some authors $[24,25]$ to denote quark-gluon plasma in this regime as "sQGP" for "strongly interacting QGP".

It is worth noting that this state of affairs has been anticipated by many authors. Whether the argument was based on the divergence of perturbative expansions [26], on phenomenological descriptions of confinement [27], on the development of effective gluon masses from plasmon modes [28] or on general principles [15], it is clear that the QGP near $T_{C}$ should not be regarded as an ideal gas of quarks and gluons.

How high a temperature is needed not just to form a quark-gluon plasma, but to approach this "weakly" interacting plasma? A calculation of the pressure of hot matter within perturbative QCD [29] is shown in Fig. 3. The pressure result oscillates significantly as one considers contributions of different orders. These oscillations are an indication that the expansion is not yielding reliable results. However at temperatures approaching 1000 times of $T_{C}\left(\approx \Lambda_{\bar{M} S}\right)$, they appear to be converging toward the Stefan-Boltzmann limit (asymptotically free partons). It is interesting that in considering the highest-order term, the results are still nonconvergent though one seems to approach the lattice calculated pressure. Unlike the case of single parton-parton scattering at zero temperature, the infrared problems of finite-temperature field theory prevent further analytic progress even for very small values of the coupling constant 


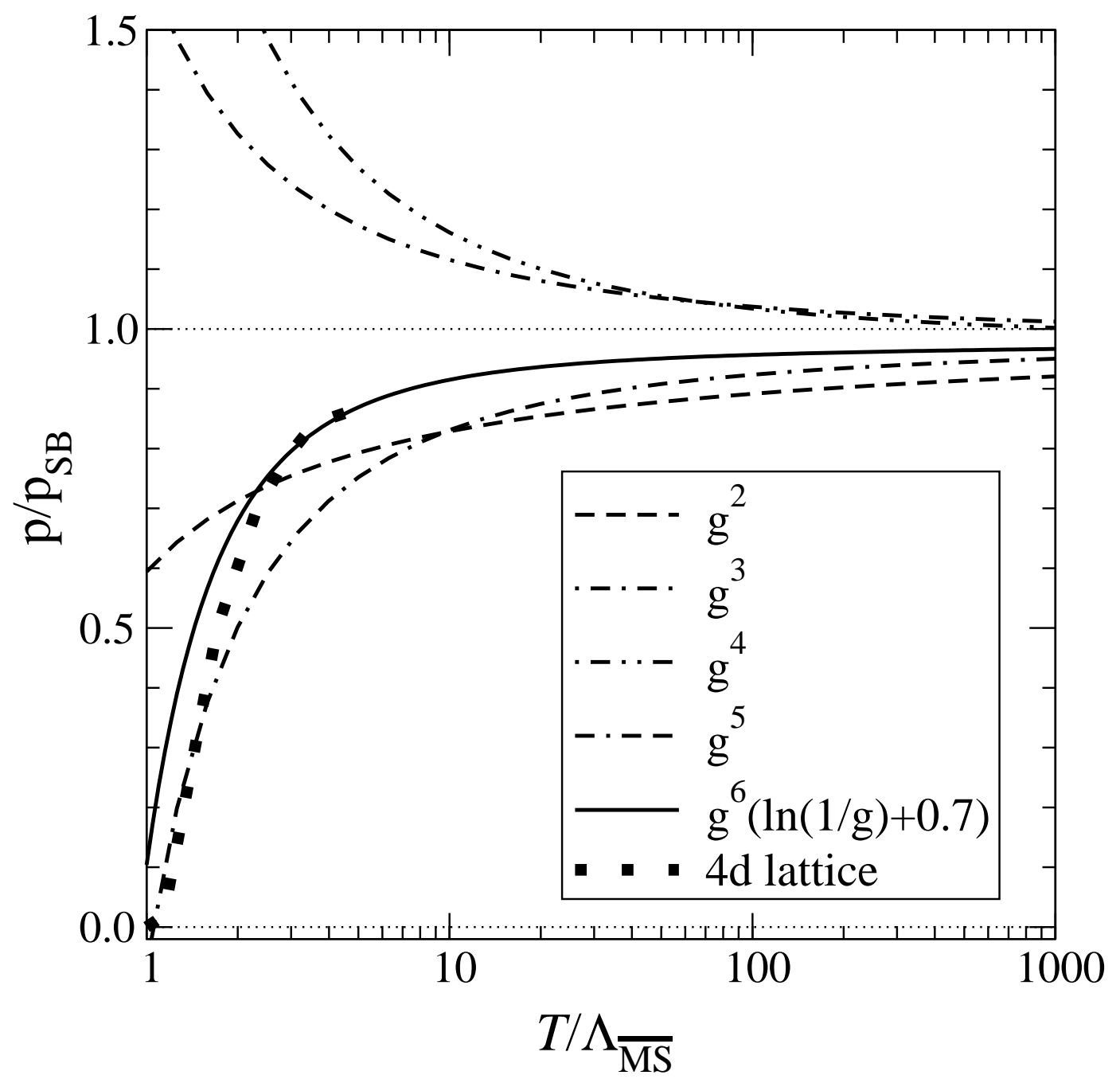

Fig. 3. Perturbative QCD results for the pressure as a function of temperature at various orders normalized to the Stefan-Boltzmann value $p_{S B}[29]$.

$[29,30,31]$.

The goal of relativistic heavy ion physics is the experimental study of the nature of QCD matter under conditions of extreme temperature. A great emphasis has been placed on "the discovery of the quark-gluon plasma", where the terminology "quark-gluon plasma" is used as a generic descriptor for a system in which the degrees of freedom are no longer the color neutral hadron states observed as isolated particles and resonances. This definition is limited since high-energy proton-proton reactions cannot be described purely in terms of color-neutral hadrons, but rather require analysis of the underlying partonic interactions. The hoped-for essential difference in heavy ion collisions is the dominance of the partonic-level description for essentially all momentum scales and over nuclear size distances. Beyond this simple criterion, in order to characterize the produced system as a state of matter it is necessary to establish that these non-hadronic degrees of freedom form a statistical ensemble, 
so that concepts such as temperature, chemical potential and flow velocity apply and the system can be characterized by an experimentally determined equation of state. Additionally, experiments eventually should be able to determine the physical characteristics of the transition, for example the critical temperature, the order of the phase transition, and the speed of sound along with the nature of the underlying quasi-particles. While at (currently unobtainable) very high temperatures $T \gg T_{c}$ the quark-gluon plasma may act as a weakly interacting gas of quarks and gluons, in the transition region near $T_{c}$ the fundamental degrees of freedom may be considerably more complex. It is therefore appropriate to argue that the quark-gluon plasma must be defined in terms of its unique properties at a given temperature. To date the definition is provided by lattice QCD calculations. Ultimately we would expect to validate this by characterizing the quark-gluon plasma in terms of its experimentally observed properties. However, the real discoveries will be of the fascinating properties of high temperature nuclear matter, and not the naming of that matter.

\subsection{Experimental Program}

The theoretical discussion of the nature of hadronic matter at extreme densities has been greatly stimulated by the realization that such conditions could be studied via relativistic heavy ion collisions [32]. Early investigations at the Berkeley Bevalac (c. 1975-1985), the BNL AGS (c. 1987-1995) and the CERN SPS (c. 1987-present) have reached their culmination with the commissioning of BNL's Relativistic Heavy Ion Collider (RHIC), a dedicated facility for the study of nuclear collisions at ultra-relativistic energies [33].

The primary goal of RHIC is the experimental study of the QCD phase transition. The 2002 Long-Range Plan for Nuclear Science [34] clearly enunciates this objective:

...the completion of RHIC at Brookhaven has ushered in a new era. Studies are now possible of the most basic interactions predicted by QCD in bulk nuclear matter at temperatures and densities great enough to excite the expected phase transition to a quark-gluon plasma. As the RHIC program matures, experiments will provide a unique window into the hot $Q C D$ vacuum, with opportunities for fundamental advances in the understanding of quark confinement, chiral symmetry breaking, and, very possibly, new and unexpected phenomena in the realm of nuclear matter at the highest densities.

The RHIC accelerator and its four experiments were commissioned and brought online in the summer of 2000. The initial operation of both RHIC and the experiments has been remarkably successful. In these first three years the ac- 
celerator has collided, and the experiments have acquired data on, $\mathrm{Au}+\mathrm{Au}$ collisions at five energies, an essential $p+p$ baseline data set, and a critical $d+\mathrm{Au}$ comparison. The analyses of these various systems have resulted in a correspondingly rich abundance of results, with over 90 publications in the refereed literature.

It is therefore appropriate to reflect on the physics accomplishments to date, with a particular emphasis on their implications for the discovery of a new state of matter. At the same time, it is essential to identify those features of the data (if any) that are at odds with canonical descriptions of the produced matter, to specify those crucial measurements which remain to be made, and to outline a program for continued exploration and characterization of strongly interacting matter at RHIC. The PHENIX collaboration [35] has performed such an assessment; this document represents a summary of its findings.

The PHENIX Conceptual Design Report [36], submitted to BNL/RHIC management on January 29th, 1993, outlined a comprehensive physics program focused on the search for and characterization of new states of nuclear matter. The measurement of electromagnetic probes and high-transverse-momentum phenomena formed a major thrust of the proposed program. It was also realized that the measurement of global variables and soft identified hadron spectra in the same apparatus was essential to the goal of understanding the evolution of the produced matter over all relevant timescales. These diverse criteria required combining an unprecedented number of subsystems together with a high-bandwidth trigger and data-acquisition system into an integrated detector design. Particular attention was given to minimizing the conflicting design criteria of the central arm spectrometers, with their requirement for minimal mass in the aperture, and those of the muon spectrometers which require maximal absorption of the incident hadron flux. The data acquisition and trigger system was designed to accommodate the great variety of interaction rates and event sizes provided by RHIC. Every effort was made to provide for future upgrades, both in the geometry of the experiment and in the architecture and design parameters of the read-out system.

The published PHENIX results of $\mathrm{Au}+\mathrm{Au}$ collision at a center-of-mass energy per nucleon pair, $\sqrt{s_{N N}}$, of $130 \mathrm{GeV}[37,38,39,40,41,42,43,44,45,46,47,48]$ and at $\sqrt{s_{N N}}=200 \mathrm{GeV}[49,50,51,52,53,54,55,56,57,58,59], p+p$ collisions at $\sqrt{s}=200 \mathrm{GeV}[60,61,62,63]$, and $d+\mathrm{Au}$ at $\sqrt{s_{N N}}=200 \mathrm{GeV}[64,65]$ clearly demonstrate that PHENIX's goal to make high-quality measurements in both hadronic and leptonic channels for collisions ranging from $p+p$ to $\mathrm{Au}+\mathrm{Au}$ has been realized. A summary of these results illustrates this point:

- First measurement of the dependence of the charged particle pseudo-rapidity density [37] and the transverse energy [38] on the number of participants in $\mathrm{Au}+\mathrm{Au}$ collisions at $\sqrt{s_{N N}}=130 \mathrm{GeV}$; systematic study of the centrality 
and $\sqrt{s_{N N}}$ dependence of $d E_{T} / d \eta$ and $d N_{c h} / d \eta[59]$.

- Discovery of suppressed production for $\pi^{0}$ 's and charged particles at high $p_{T}$ in $\mathrm{Au}+\mathrm{Au}$ collisions at $\sqrt{s_{N N}}=130 \mathrm{GeV}[39]$ and a systematic study of the scaling properties of the suppression [47]; extension of these results to much higher transverse momenta in $\mathrm{Au}+\mathrm{Au}$ collisions at $\sqrt{s_{N N}}=200 \mathrm{GeV}$ $[49,53]$.

- Co-discovery (together with BRAHMS [66], PHOBOS [67] and STAR [68]) of absence of high- $p_{T}$ suppression in $d+\mathrm{Au}$ collisions at $\sqrt{s_{N N}}=200 \mathrm{GeV}$ [64].

- Discovery of the anomalously large proton and anti-proton yields at intermediate transverse momentum in $\mathrm{Au}+\mathrm{Au}$ collisions at $\sqrt{s_{N N}}=130 \mathrm{GeV}$ through the systematic study of $\pi^{ \pm}, K^{ \pm}, p$ and $\bar{p}$ spectra [40]; study of the scaling properties of the proton and anti-proton yields in $\mathrm{Au}+\mathrm{Au}$ collisions at $\sqrt{s_{N N}}=200 \mathrm{GeV}[52]$;

- Measurement of $\Lambda$ 's and $\bar{\Lambda}$ 's in $\mathrm{Au}+\mathrm{Au}$ collisions at $\sqrt{s_{N N}}=130 \mathrm{GeV}$ [43]; measurement of $\phi$ 's at $\sqrt{s_{N N}}=200 \mathrm{GeV}$ [69]; measurement of deuteron and anti-deuteron spectra at $\sqrt{s_{N N}}=200 \mathrm{GeV}[58]$.

- Measurement of Hanbury-Brown-Twiss (HBT) correlations in $\pi^{+} \pi^{+}$and $\pi^{-} \pi^{-}$pairs in $\mathrm{Au}+\mathrm{Au}$ collisions at $\sqrt{s_{N N}}=130 \mathrm{GeV}$ [41] and $200 \mathrm{GeV}$ [56], establishing that the "HBT puzzle" of $R_{\text {out }} \approx R_{\text {side }}$ extends to high pair momentum.

- First measurement of single electron spectra in $\mathrm{Au}+\mathrm{Au}$ collisions at $\sqrt{s_{N N}}=$ $130 \mathrm{GeV}$, suggesting that charm production scales with the number of binary collisions [42]; measurement of centralty dependence of charm production in $\mathrm{Au}+\mathrm{Au}$ collisions at $\sqrt{s_{N N}}=200 \mathrm{GeV}$ [57].

- Sensitive measures of charge fluctuations [44] and fluctuations in mean $p_{T}$ and transverse energy per particle $[45,55]$ in $\mathrm{Au}+\mathrm{Au}$ collisions at $\sqrt{s_{N N}}=$ $130 \mathrm{GeV}$ and $200 \mathrm{GeV}$.

- Measurements of elliptic flow for charged particles from $\mathrm{Au}+\mathrm{Au}$ collisions at $\sqrt{s_{N N}}=130 \mathrm{GeV}[46]$ and $62 \mathrm{GeV}$ to $200 \mathrm{GeV}$ [70] and identified charged hadrons from $\mathrm{Au}+\mathrm{Au}$ collisions at $\sqrt{s_{N N}}=200 \mathrm{GeV}[50]$.

- Extensive study of hydrodynamic flow, particle yields, ratios and spectra from $\mathrm{Au}+\mathrm{Au}$ collisions at $\sqrt{s_{N N}}=130 \mathrm{GeV}$ [48] and $200 \mathrm{GeV}$ [54].

- First observation of $J / \psi$ production in $\mathrm{Au}+\mathrm{Au}$ collisions at $\sqrt{s_{N N}}=200$ $\mathrm{GeV}[51]$.

- Measurement of the nuclear modification factor for hadrons at forward and backward rapidities in $d+\mathrm{Au}$ collisions at $\sqrt{s_{N N}}=200 \mathrm{GeV}$ [65].

- First measurement of the jet structure of baryon excess in $\mathrm{Au}+\mathrm{Au}$ collisions at $\sqrt{s_{N N}}=200 \mathrm{GeV}[71]$.

- First measurement of elliptic flow of single electrons from charm decay in $\mathrm{Au}+\mathrm{Au}$ collisions at $\sqrt{s_{N N}}=200 \mathrm{GeV}[72]$.

- First measurement of direct photons in $\mathrm{Au}+\mathrm{Au}$ collisions at $\sqrt{s_{N N}}=200$ $\mathrm{GeV}[73]$.

- Measurement of crucial baseline data on $\pi^{0}$ spectra [60], direct photon producion [63], and $J / \psi$ production [61] in $p+p$ collisions at $\sqrt{s}=200 \mathrm{GeV}$. 
- First measurement of the double longitudinal spin asymmetry $A_{L L}$ in $\pi^{0}$ production for polarized $p+p$ collisions at $\sqrt{s}=200 \mathrm{GeV}[62]$.

These publications encompass physics from the barn to the picobarn level; their very breadth precludes a detailed presentation here. These data, together with a rich program of future RHIC measurements, will allow us to address many of the features that would characterize a quark-gluon plasma:

- Temperature

- Parton number density

- Energy density

- Opacity

- Collective behavior

- Thermalization leading to the quark-gluon phase

- Deconfinement

- Number and nature of degrees of freedom

- Recombination of quarks and gluons to form final-state hadrons

- Chiral symmetry restoration

- Time evolution of system parameters

- Equation of state

- Color and thermal transport properties

- Critical behavior

As emphasized above, the present PHENIX data set from RHIC runs in year 2000 to 2003 already provides an extensive set of measurements on global variables: (transverse energy and multiplicity, elliptic flow); correlations and fluctuations: (fluctuations in charge and $\left\langle p_{T}\right\rangle$, HBT measurements), hadron spectra: (low- $p_{T}$ single-hadron spectra and radial flow, particle ratios, resonances, anomalous $p / \pi$ ratio at intermediate $p_{T}$ ); high- $p_{T}$ physics: (high- $p_{T}$ singles spectra, suppression phenomena in $A+A$, nonsuppression in $d+A$, high- $p_{T}$ two-particle correlations, nuclear suppression/enhancement in forward/backward directions), heavy flavor production: (charm, $J / \psi$ ), and electromagnetic probes: (direct photons). However, an important conclusion of this report is that systematic studies of these observables (vs. collision species and energy) are needed to extract unambiguous information on most of these features.

\subsection{Organization of this Document}

As a result, this paper concentrates on those aspects of the present data that address the broad features of energy density, thermalization, deconfinement and critical behavior. The focus in most cases will be on the data of the PHENIX experiment, but the data of the other RHIC experiments will be 
cited to support and to extend the discussion ${ }^{3}$. The experimental tools that allow the systematic study of all phenomena as a function of the inferred impact parameter are presented in the context of hard-scattering phenomena. These methods and the associated data are then used to discuss the experimental evidence for the formation of a state of high-density matter. The measured abundances, spectra and flow patterns are used to analyze the degree of thermalization and collectivity in the produced matter. These results are then examined for evidence establishing the role of deconfined quarks and gluons in the produced system, along with the implications for its description as a quark-gluon plasma. A concluding section summarizes the findings and identifies key future measurements required to further refine our observations.

\section{ENERGY DENSITY AND $E_{T}, N_{C H}$}

A prerequisite for creating a quark-gluon plasma is producing a system with sufficiently large energy density. From both elementary estimates [22] and from extensive numerical studies in lattice QCD [11,10], the required density is known to be on the order of $1 \mathrm{GeV} / \mathrm{fm}^{3}$. Establishing that this energy density is created in RHIC collisions is a basic ingredient in establishing the creation of a QGP at RHIC.

In this section we explore what can be deduced about the energy densities achieved in RHIC $A+A$ collisions from measurements of the global transverse energy and multiplicity. In later sections these estimates will be compared to densities inferred from hydrodynamics-based models (Section 3) and from jet quenching evidence (Section 6).

Specifically, we will address three different energy density estimates, and introduce two distinct time scales: (i) The peak general energy density that is achieved when the incoming nuclei overlap; (ii) The peak formed energy density involving created particles at proper time $\tau_{\text {Form }}$; and (iii) The peak thermalized energy density present at proper time $\tau_{\text {Therm }}$ when local thermal equilibrium is first achieved (assuming that this occurs). The values and time scales for formed and thermalized energy densities are indicated schematically in Fig 4; detailed explanations follow in Sections 2.3 and 2.4.

In this Section we will also review data on overall particle multiplicities, and through them distinguish between different models of the initial particle production.

3 An underappreciated aspect of the RHIC program is the excellent agreement between the various experiments in almost all measured channels. 


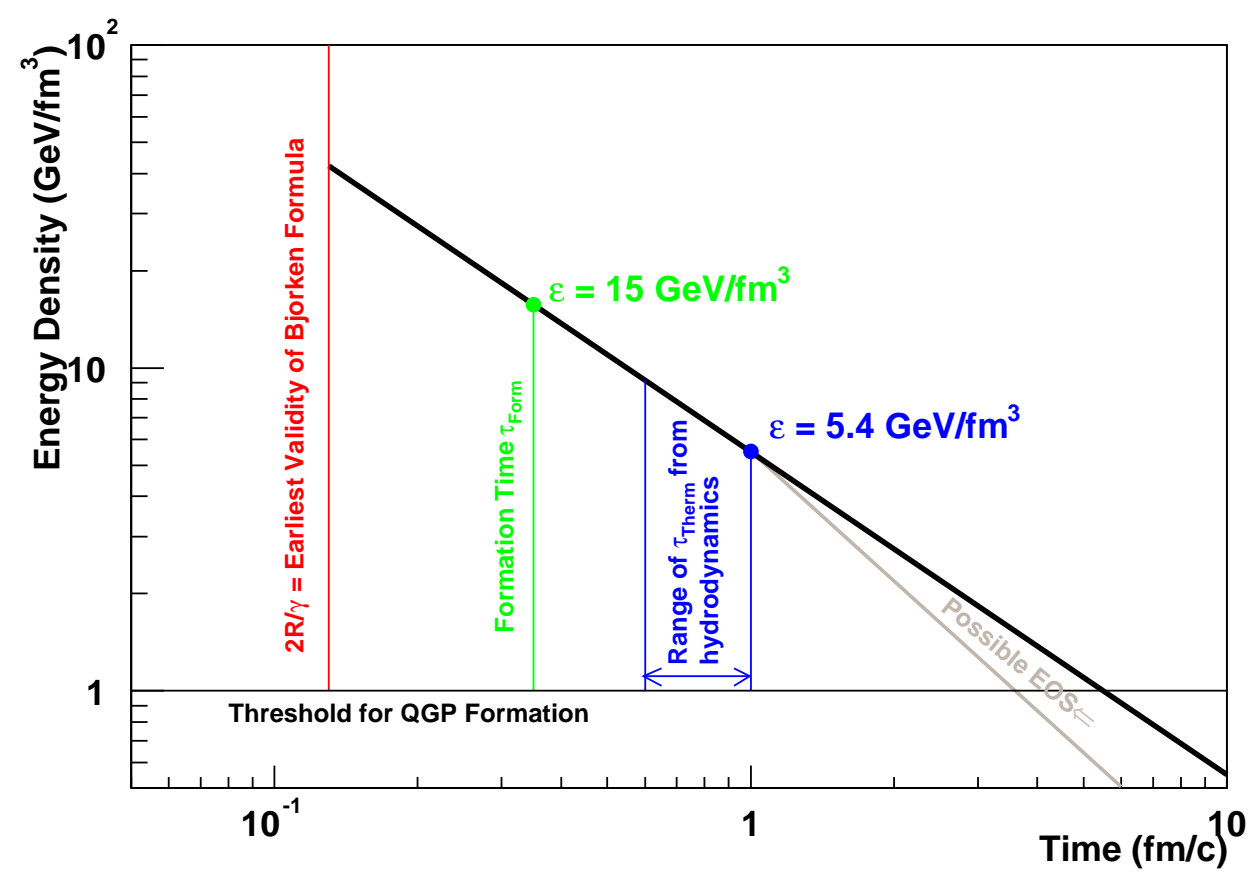

Fig. 4. Schematic drawing of the time and energy density scales derived through the Bjorken picture.

\subsection{General Energy Density}

The simplest definition of "energy density" is the total mass-energy within some region of space divided by the volume of that region, as seen at some instant of time in some Lorentz frame. However, this definition is not satisfactory since we can "trivially" raise any simple energy density by viewing the system in a different frame. For example, a static system with constant energy density $\rho_{0}$ in its rest frame - say, a gold nucleus - will appear to have energy density $\gamma^{2} \rho_{0}$ when viewed in a frame boosted by Lorentz $\gamma$. Accordingly, we can only calculate a meaningful energy density $\langle\varepsilon\rangle$ as mass-energy/volume for some region in the case when the total momentum in the region is zero.

Now let us imagine a symmetric RHIC $A+A$ collision at a moment when the two original nuclei are overlapping in space, as seen in the laboratory/centerof-mass frame. The total momentum in any overlap region is zero by symmetry, so we can calculate a meaningful - if short-lived - energy density for such a region. If each nucleus has energy density $\rho_{0}$ in its rest frame then the total energy density in the overlap region is just $\langle\varepsilon\rangle=2 \rho_{0} \gamma^{2}$. If we take a nominal $\rho_{0}=0.14 \mathrm{GeV} / \mathrm{fm}^{3}$ for a nucleus at rest and $\gamma=106$ for a full-energy RHIC collision, then the result for the peak general energy density is $\langle\varepsilon\rangle=3150$ $\mathrm{GeV} / \mathrm{fm}^{3}$. This is a spectacularly, almost absurdly high number on the scale of $\sim 1 \mathrm{GeV} / \mathrm{fm}^{3}$ associated with the familiar transition described by lattice 
QCD.

This energy density is of course artificial, in that it would be temporarily present even in the case of no interactions between the two nuclei. It is instructive to consider the (again artificial) case where the nucleons in the two nuclei have only elastic interactions. Then the time during which a high energy density is present over any volume cannot last longer than $t=2 R / \gamma$, where $R$ is the rest-frame radius of the nucleus. With $R=7 \mathrm{fm}$ for $\mathrm{Au}$ this time is only $0.13 \mathrm{fm} / c$ at RHIC, and after this time all energy densities will fall precipitously back to $\rho_{0}$ if no secondary particles are created. The scale of this interval is so short that a scattering cannot even be said to have occurred within that volume unless its momentum transfer scale $Q$ exceeds at least 1.5 $\mathrm{GeV} / c$, or more. Accordingly, we will turn our attention instead to energy densities involving only produced particles, as the potential source for a QCD transition.

\subsection{Formed Energy Density}

In any frame (not just the center-of-mass frame) where the two incoming nuclei have very high energies the region when/where the nuclei overlap will be very thin in the longitudinal direction and very short in duration. In this limit, then, it is fair to describe all secondary produced particles as having been radiated out from a very thin "disk", and that they are all created at essentially the same time. These realizations lead directly to the picture described by Bjorken [74], whose original diagram is reproduced in Fig. 5 and whose derivation we retrace briefly here.

Once the beam "pancakes" recede after their initial overlap, the region between them is occupied by secondaries at intermediate rapidities. We can calculate the local energy density of these created particles if we make one further assumption: that the secondaries can be considered "formed" at some proper time $\tau_{\text {Form }}$ after they are radiated out from the thin source disk.

Our region of interest, in any frame, will be a slab perpendicular to the beam direction, with longitudinal thickness $d z$, with one face on the "source" plane in this frame, and transverse extent with area $A$ covering the nuclear overlap region ${ }^{4}$. At time $t=\tau_{\text {Form }}$ this volume will contain all the (now-formed) particles with longitudinal velocities $0 \leq \beta_{\|} \leq d z / \tau_{\text {Form }}$ (since we assume that the particles cannot scatter before they are formed!). We can then write this number of particles as $d N=\left(d z / \tau_{\text {Form }}\right) \frac{d N}{d \beta_{\|}}$, or equivalently $d N=\left(d z / \tau_{\text {Form }}\right) \frac{d N}{d y}$,

4 The region described here corresponds to half the shaded region shown in Fig. 5. Since $\beta_{\|} \simeq 0$ for particles near the source location, this is an appropriate region over which we can calculate a meaningful energy density. 


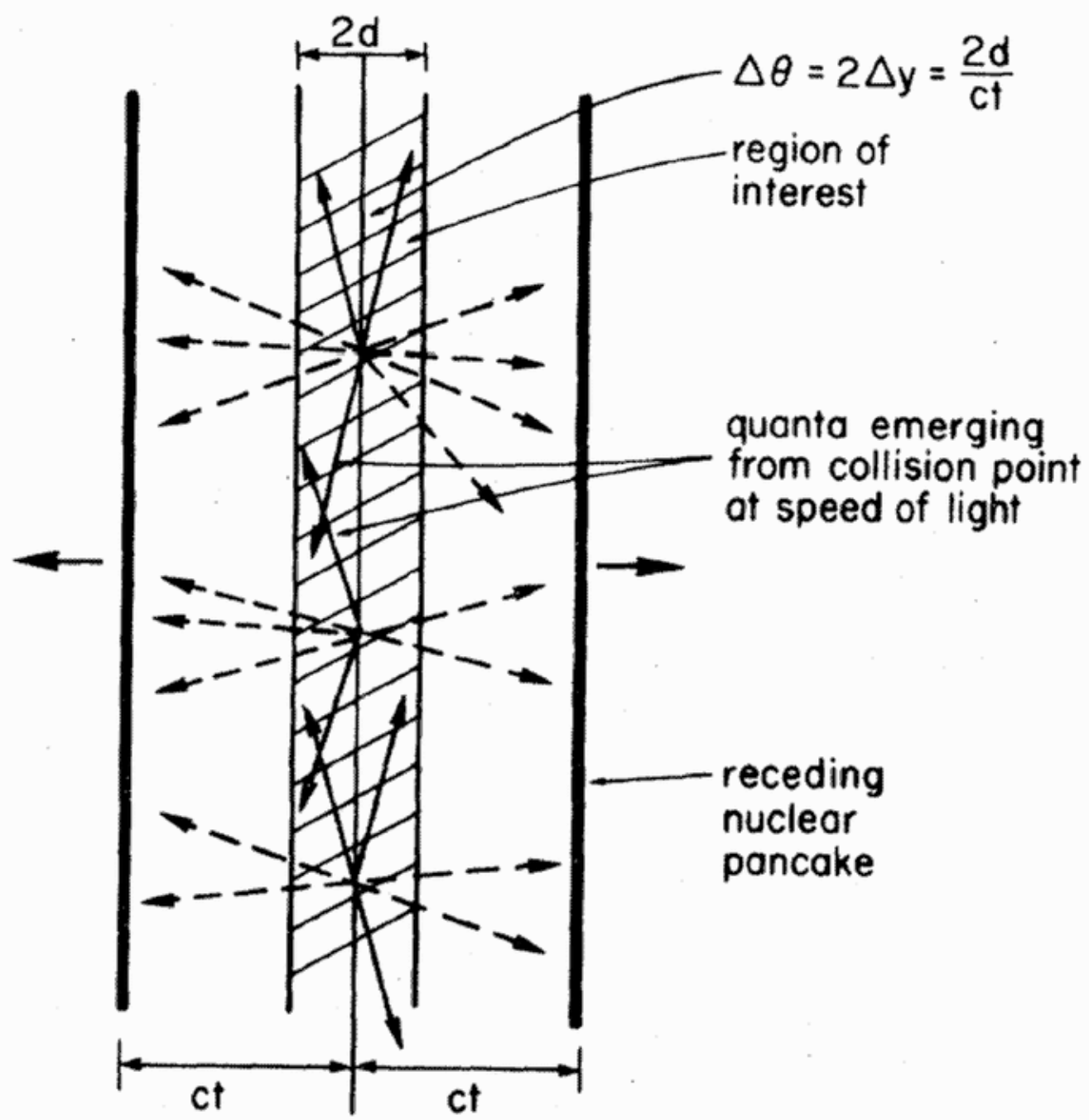

Fig. 5. Figure from Bjorken [74] illustrating the geometry of initially produced particles at a time $t$ after the overlap of the incoming nuclei in some frame. The picture is valid in any frame in which the incoming nuclei have very high energies and so are highly Lorentz contracted.

where $y$ is longitudinal rapidity, since $d y=d \beta_{\|}$at $y=\beta_{\|}=0$. If these particles have an average total energy $\left\langle m_{T}\right\rangle$ in this frame $\left(E=m_{T}\right.$ for particles with no longitudinal velocity), then the total energy divided by the total volume of the slab at $t=\tau_{\text {Form }}$ is just

$$
\begin{aligned}
\left\langle\varepsilon\left(\tau_{\text {Form }}\right)\right\rangle & =\frac{d N\left\langle m_{T}\right\rangle}{d z A}=\frac{d N\left(\tau_{\text {Form }}\right)}{d y} \frac{\left\langle m_{T}\right\rangle}{\tau_{\text {Form }} A} \\
& =\frac{1}{\tau_{\text {Form }} A} \frac{d E_{T}\left(\tau_{\text {Form }}\right)}{d y}
\end{aligned}
$$


where we have equated $\frac{d E_{T}}{d y}=\left\langle m_{T}\right\rangle \frac{d N}{d y}$ and emphasized that Eq. 5 is true for the transverse energy density present at time $t=\tau_{\text {Form }}$.

Equation 5 here is essentially identical ${ }^{5}$ to Eq. 4 of Bjorken's result [74], and so is usually referred to as the Bjorken energy density $\varepsilon_{B j}$. It should be valid as a measure of peak energy density in created particles, on very general grounds and in all frames, as long as two conditions are satisfied: (1) A finite formation time $\tau_{\text {Form }}$ can meaningfully be defined for the created secondaries; and (2) The thickness/ "crossing time" of the source disk is small compared to $\tau_{\text {Form }}$, that is, $\tau_{\text {Form }} \gg 2 R / \gamma$. In particular, the validity of Eq. 5 is completely independent of the shape of the $d E_{T}\left(\tau_{F o r m}\right) / d y$ distribution to the extent that $\beta_{\|}$is infinitesimally small in a comoving frame; a plateau in $d E_{T} / d y$ is not required. For present practical purposes, we will consider condition (2) above to be satisfied as long as $\tau_{\text {Form }}>2 R / \gamma$ is true, corresponding to $\tau_{\text {Form }}>0.13$ $\mathrm{fm} / c$ for full-energy $\mathrm{Au}+\mathrm{Au}$ collisions at RHIC.

Bjorken's original motivation was to estimate, in advance of data, the energy densities that would be reached in high-energy $A+A$ collisions, using knowledge of $p(\bar{p})+p$ collisions to estimate $\left\langle m_{T}\right\rangle$ and $d N / d y$, and choosing $\tau_{\text {Form }} \sim 1$ $\mathrm{fm} / c$ without any particular justification other than as an order-of-magnitude estimate. With $A+A$ collision data in hand, attempts have been made to use Eq. 5 to estimate the energy densities that are actually achieved in the collisions. Historically, $\varepsilon_{B j}$ has been calculated using the final-state $d E_{T} / d y$ and simply inserting a nominal value of $1 \mathrm{fm} / c$ for $\tau_{F o r m}$. In addition, fixed target experiments have been using $d E_{T} / d \eta$ as an estimate for $d E_{T} / d y$, which is a good approximation for these experiments; at RHIC a correction is made for the Jacobian $d y / d \eta$ which is important for a collider geometry. These "nominal Bjorken energy density" estimates, which we term $\varepsilon_{B j}^{\text {Nominal }}$, range for central event samples from about $1.5 \mathrm{GeV} / \mathrm{fm}^{3}$ in $\mathrm{Au}+\mathrm{Au}$ collisions at AGS energies [75] $\left(\sqrt{s_{N N}}=5 \mathrm{GeV}\right)$, to about $2.9 \mathrm{GeV} / \mathrm{fm}^{3}$ in $\mathrm{Pb}+\mathrm{Pb}$ collisions at SPS energies $[76,38]\left(\sqrt{s_{N N}}=17 \mathrm{GeV}\right.$; and see also [59]) to about $5.4 \mathrm{GeV} / \mathrm{fm}^{3}$ in $\mathrm{Au}+\mathrm{Au}$ collisions at full RHIC energy $[59]\left(\sqrt{s_{N N}}=200 \mathrm{GeV}\right)$.

It has often been noted that all of these values are similar to, or higher than, the $1 \mathrm{GeV} / \mathrm{fm}^{3}$ scale required for the QCD transition. However, we cannot take these $\varepsilon_{B j}^{N o m i n a l}$ estimates seriously as produced energy densities without some justification for the value of $1 \mathrm{fm} / c$ taken for $\tau_{\text {Form }}$. An indication of potential problems with this choice arises immediately when considering AGS $\mathrm{Au}+\mathrm{Au}$ and SPS $\mathrm{Pb}+\mathrm{Pb}$ collisions, where the center-of-mass "crossing times" $2 R / \gamma$ are $5.3 \mathrm{fm} / c$ and $1.6 \mathrm{fm} / c$ respectively, which implies that this choice for $\tau_{\text {Form }}=1 \mathrm{fm} / c$ actually violates the validity condition $\tau_{\text {Form }}>2 R / \gamma$ we set for the use of Eq. 5. So we will deprecate the use of $\varepsilon_{B j}^{N o m i n a l}$ as an quantitative estimate of actual produced energy density, and instead treat it only as a

$\overline{5}$ A (well-known) factor of 2 error appears in the original. 
compact way of comparing $d E_{T} / d \eta$ measurements across different systems, centralities and beam energies.

\subsection{Realistic $\tau_{\text {Form }}$ and $\varepsilon_{B j}$ estimates}

Can we justify a better estimate for $\tau_{\text {Form }}$ ? We might say, on general quantum mechanical grounds, that in a frame where its motion is entirely transverse a particle of energy $m_{T}$ can be considered to have "formed" after a time $t=\hbar / m_{T}$ since its creation in that frame. To estimate the average transverse mass, we can use the final-state $d E_{T} / d \eta$ to estimate $d E_{T}\left(\tau_{F o r m}\right) / d y$ and, correspondingly, use the final-state $d N / d \eta$ as an estimate for $d N\left(\tau_{\text {Form }}\right) / d y$ to obtain

$$
\left\langle m_{T}\right\rangle=\frac{d E_{T}\left(\tau_{\text {Form }}\right) / d y}{d N\left(\tau_{\text {Form }}\right) / d y} \simeq \frac{d E_{T} / d \eta}{d N / d \eta}(\text { Final state }) .
$$

PHENIX has measured the ratio of final-state transverse-energy density to charged-particle density, each per unit pseudorapidity, and the results are shown in Fig. 6. For a wide range of centralities the ratio is remarkably constant at about $0.85 \mathrm{GeV}$ for full-energy central $\mathrm{Au}+\mathrm{Au}$ collisions and shows very little change with beam energy, decreasing to only $0.7 \mathrm{GeV}$ when $\sqrt{s_{N N}}$ is decreased by an order of magnitude down to $19.6 \mathrm{GeV}$.

If we approximate $d N^{C h} / d \eta=(2 / 3) d N / d \eta$ in the final state then Eq. 6 would imply $\left\langle m_{T}\right\rangle \simeq 0.57 \mathrm{GeV}$ and corresponding $\tau_{\text {Form }} \simeq 0.35 \mathrm{fm} / c$, a value shorter than the "nominal" $1 \mathrm{fm} / c$ but still long enough to satisfy our validity condition $\tau_{\text {Form }}>2 R / \gamma$ at RHIC. Inserting this value into Eq. 5, along with the highest $d E_{T} / d \eta=600 \mathrm{GeV}$ for $0-5 \%$ central events as measured by PHENIX [59], yields a value of $\langle\varepsilon\rangle=15 \mathrm{GeV} / \mathrm{fm}^{3}$ for the energy density in initially produced, mid-rapidity particles in a central $\mathrm{RHIC} \mathrm{Au}+\mathrm{Au}$ collision, that is, roughly 100 times the mass-energy density of cold nuclear matter.

It is important to note that this large value of the energy density as obtained from Eq. 5 represents a conservative lower limit on the actual $\left\langle\varepsilon\left(\tau_{\text {Form }}\right)\right\rangle$ achieved in RHIC collisions. This follows from two observations: (1) The finalstate measured $d E_{T} / d \eta$ is a solid lower limit on the $d E_{T}\left(\tau_{F o r m}\right) / d y$ present at formation time; and (2) The final-state ratio $\left(d E_{T} / d \eta\right) /(d N / d \eta)$ is a good lower limit on $\left\langle m_{T}\right\rangle$ at formation time, and so yields a good upper limit on $\tau_{\text {Form }}$. We justify these statements as follows:

Several mechanisms are known that will decrease $d E_{T} / d y$ as the collision system evolves after the initial particle formation, while no mechanism is known that can cause it to increase (for $y=0$, at least). Therefore, its final-state 


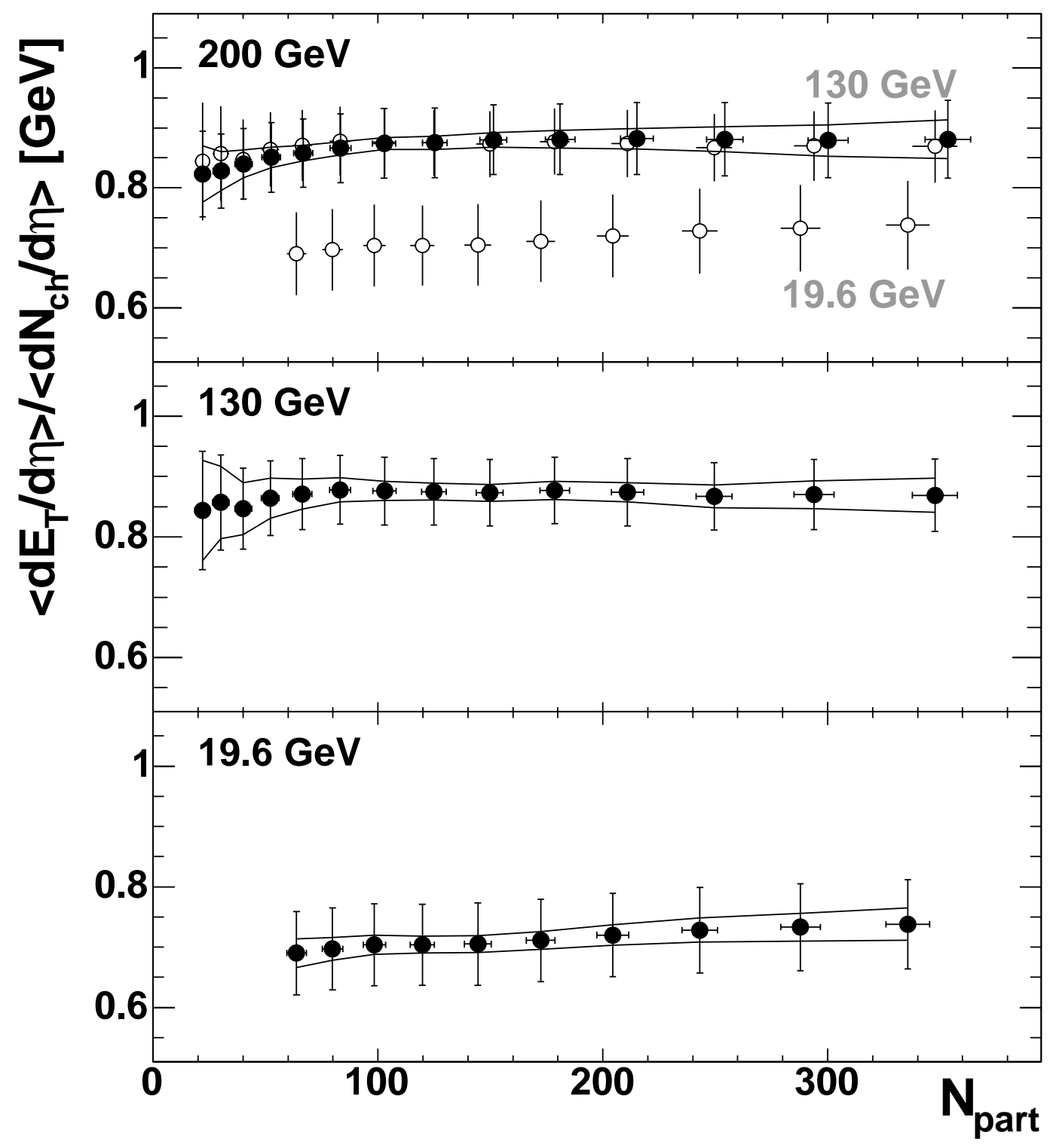

Fig. 6. The ratio of transverse energy density in pseudorapidity to charged particle density in pseudorapidity, at mid-rapidity; shown as a function of centrality, represented by the number of nucleons participating in the collision, $N_{\text {part }}$, for three different RHIC beam energies [59].

value should be a solid lower limit on its value at any earlier time. A partial list of the mechanisms through which $d E_{T} / d y$ will decrease after $t=\tau_{\text {Form }}$ includes: (i) The initially formed secondaries in any local transverse "slab" will, in a comoving frame, have all their energy in transverse motion and none in longitudinal motion; if they start to collide and thermalize, at least some of their $E_{T}$ will be converted to longitudinal modes in the local frame; (ii) Should rough local thermal equilibrium be obtained while the system's expansion is still primarily longitudinal, then each local fluid element will lose internal energy through $p d V$ work and so its $E_{T}$ will decrease; (iii) If there are 
pressure gradients during a longitudinal hydrodynamic expansion then some fluid elements may be accelerated to higher or lower rapidities; these effects are complicated to predict, but we can state generally that they will always tend to decrease $d E_{T} / d y$ where it has its maximum, namely at $y=0$. Given that we have strong evidence that thermalization and hydrodynamical evolution do occur in RHIC collisions (Section 3), it is likely that all these effects are present to some degree, and so we should suspect that final-state $d E_{T} / d \eta$ is substantially lower than $d E_{T}\left(\tau_{\text {Form }}\right) / d y$ at mid-rapidity.

Turning to our estimate of $\tau_{\text {Form }}$, the assumption that $\tau_{\text {Form }}=\hbar /\left\langle m_{T}\right\rangle$ cannot be taken as exact, even if the produced particles' $m_{T}$ 's are all identical, since "formed" is not an exact concept. However, if we accept the basic validity of this uncertainty principle argument, then we can see that the approximation in Eq. 6 provides a lower limit on $\left\langle m_{T}\right\rangle$. First, the numerator $d E_{T} / d \eta$ is a lower limit on $d E_{T}\left(\tau_{\text {Form }}\right) / d y$, as above. Second, the argument is often made on grounds of entropy conservation that the local number density of particles can never decrease [77], which would make the final-state denominator in Eq. 6 an upper limit on its early-time value.

With these limits in mind, then, it is not unreasonable for us to claim that the peak energy density of created particles reached in central $\mathrm{Au}+\mathrm{Au}$ collision at RHIC is at least $15 \mathrm{GeV} / \mathrm{fm}^{3}$, and in all likelihood is significantly higher.

\subsection{Thermalized Energy Density}

We have arrived at a reasonably solid, lower-limit estimate for the energy density in produced particles in a RHIC $\mathrm{Au}+\mathrm{Au}$ collision, and it is more than enough to drive a QCD transition. But the situation at $t=\tau_{\text {Form }}$ pictured in Fig. 5 looks nothing like local thermal equilibrium. It is an important question, then, to ask: if and when the system evolves to a state of local thermal equilibrium, is the energy density still sufficient to drive the transition to a QGP?

To answer this we begin by looking at the state of the system at $t=\tau_{\text {Form }}$ and immediately afterward. At the time they are formed the particles have sorted themselves out automatically, with all the particles on a "sheet" at a longitudinal position $z$ having the same longitudinal velocity $\beta_{\|}=z / t$; and so in the rest frame of a sheet all the sheet's particles have only transverse motion. If the particles continue free-streaming and never reinteract then the energy density will continue to fall as $\varepsilon \sim 1 / t$ and the Bjorken formula in Eq. 5 will be valid, with $t$ in place of $\tau_{\text {Form }}$, as long as the expansion is primarily longitudinal ${ }^{6}$.

\footnotetext{
$\overline{6}$ For long times $t>R$ transverse expansion will become significant and the energy
} 
For thermalization to occur the particles will have to start interacting and/or radiating. Once this happens the particles which were originally together on one "sheet" will start to spread in longitudinal velocity, though on short time scales we would expect their group average longitudinal velocity to remain the same. If the thermalization process is fast enough, then, we would expect that at time $t=\tau_{\text {Therm }}$ these groups will have formed locally equilibrated fluid elements, with a velocity profile following $\beta_{\|}^{\text {Fluid }}=z / t$. The energy density at this time will be reduced from the energy density at formation time $\varepsilon\left(\tau_{\text {Form }}\right)$ by a factor $\tau_{\text {Form }} / \tau_{\text {Therm }}$; i.e. the $\varepsilon_{B j}$ of Eq. 5 but with $\tau_{\text {Therm }}$ in place of $\tau_{\text {Form. }}$. This evolution is illustrated in Fig. 4.

Once local equilibration is achieved we would then expect the system to evolve hydrodynamically, and the behavior of $\varepsilon(t)$ will depend on the details of the local equations of state (EOS). Without knowing those details, though, we can say that in the limit of low pressure, $p / \varepsilon \sim 0$, the energy density will continue to evolve (during longitudinal expansion) as $\varepsilon \sim 1 / t$, while in the limit of high pressure, $p / \varepsilon \sim 1 / 3$, the energy density will decrease somewhat more quickly, $\varepsilon \sim 1 / t^{4 / 3}$, within a fluid element. This range of possible behaviors for $t>\tau_{\text {Therm }}$ is indicated schematically in Fig. 4 .

A direct theoretical determination of $\tau_{\text {Therm }}$ would require a detailed description of both the parton-parton interactions and the resulting evolution of the system density. However, other lines of reasoning may provide information on $\tau_{\text {Therm }}$. For example, it has been argued [78] that the strong elliptic flow in RHIC collisions can be taken as evidence for fast thermalization (see Section 3.3). In a hydrodynamic picture the source of elliptic flow is the spatial anisotropy of the energy density in the transverse plane at the time hydrodynamics becomes valid. If local equilibration and the onset of hydrodynamics is delayed because interactions between the initially produced particles are weak at first, then the spatial anisotropy which could give rise to elliptic flow will be reduced (see Fig. 14). This, in effect, limits how high $\tau_{\text {Therm }}$ can be if hydrodynamics is the mechanism for generating elliptic flow.

We can see from Table 1 in Section 3.5 that hydrodynamical models typically require quite short thermalization times, in the range of $0.6-1.0 \mathrm{fm} / c$, in order to reproduce the magnitude of elliptic flow which is observed at RHIC. If we take this range as typical of what hydrodynamics would imply for $\tau_{\text {Therm }}$, then we can calculate the corresponding "typical" implied energy densities at thermalization time as in range of $5.4 \mathrm{GeV} / \mathrm{fm}^{3}$ to $9.0 \mathrm{GeV} / \mathrm{fm}^{3}$. These densities are well above that required to drive the QCD transition, so the combination of our transverse energy measurements and the fast thermalization times from hydrodynamics can be taken, to some degree, as evidence that conditions to create the equilibrated upper phase of QCD matter are achieved at RHIC.

$\overline{\text { density }}$ will decrease as $\varepsilon \sim 1 / t^{3}$. 


\subsection{What Are the Initial Quanta?}

With our extensive use of the picture in Fig. 5 it is only natural to ask, "What are these initially produced particles?" that Bjorken referred to, nonspecifically, as "quanta". What models do we have for initial production, and what can we say about them using our data on $E_{T}$ and multiplicity?

The simplest assumption is that the initially produced particles in a RHIC collision are scattered partons at mid- to low- $p_{T}$, traditionally known as "minijets". For a long period in advance of RHIC data, it was widely expected that mini-jets would be the dominant channel for $E_{T}$ and particle production, and this led to two further, general expectations: first, that multiplicity and $E_{T}$ per interacting nucleon would go up sharply at collider energies, as compared to fixed-target energies, since jet and mini-jet cross sections are increasing quickly with $\sqrt{s}$ (see Fig. 7 ); and, secondly, that $E_{T}$ and multiplicity per participating nucleon would increase steeply in more central events, since the rate of hard pQCD scatterings goes up faster with centrality than does the number of interacting nucleons.

It was therefore quite surprising when the first RHIC data $[80,37,38]$ showed lower multiplicities than had been predicted from mini-jet models, and only a modest increase in $E_{T}$ and multiplicity per participant as functions of centrality. Compared to the sharp rise, shown in Fig. 7, predicted by straightforward factorized pQCD, it was clear that some mechanism must be acting at RHIC energies to restrict, or regulate, particle production $[81,79]$.

pQCD-based models have parameters regulating the momentum scales; these include a lower-momentum cutoff, and the factorization and fragmentation scales. Figure 8 shows that the pQCD-based HIJING model, circa 2002, was able to reproduce $130 \mathrm{GeV}$ and $200 \mathrm{GeV} d N_{c h} / d \eta$ reasonably well. However, in that model jet production via hard scattering is an important mechanism for particle production, and the combination of the $\sqrt{s}$ dependence of hardscattering cross sections with the growth of the nuclear overlap with centrality causes the model to predict an increase in the ratio between the two data sets with centrality. The observed ratio is, instead, quite constant. Thus the authors found it necessary to introduce a centrality-dependent shadowing to regulate the jet growth [79].

An alternative to models which use collinearly factorized pQCD is found in the "color glass condensate" picture, in which the gluon population of low$x$, low- $p_{T}$ states in the initial nuclear wave function is limited by transverse overlap and fusion of these low- $p_{T}$ gluons. The phase-space density saturates because of the competition between extra gluon radiation from higher- $x$ gluons and non-linear fusion of the gluons at high density. $\mathrm{Au}+\mathrm{Au}$ collisions are then 


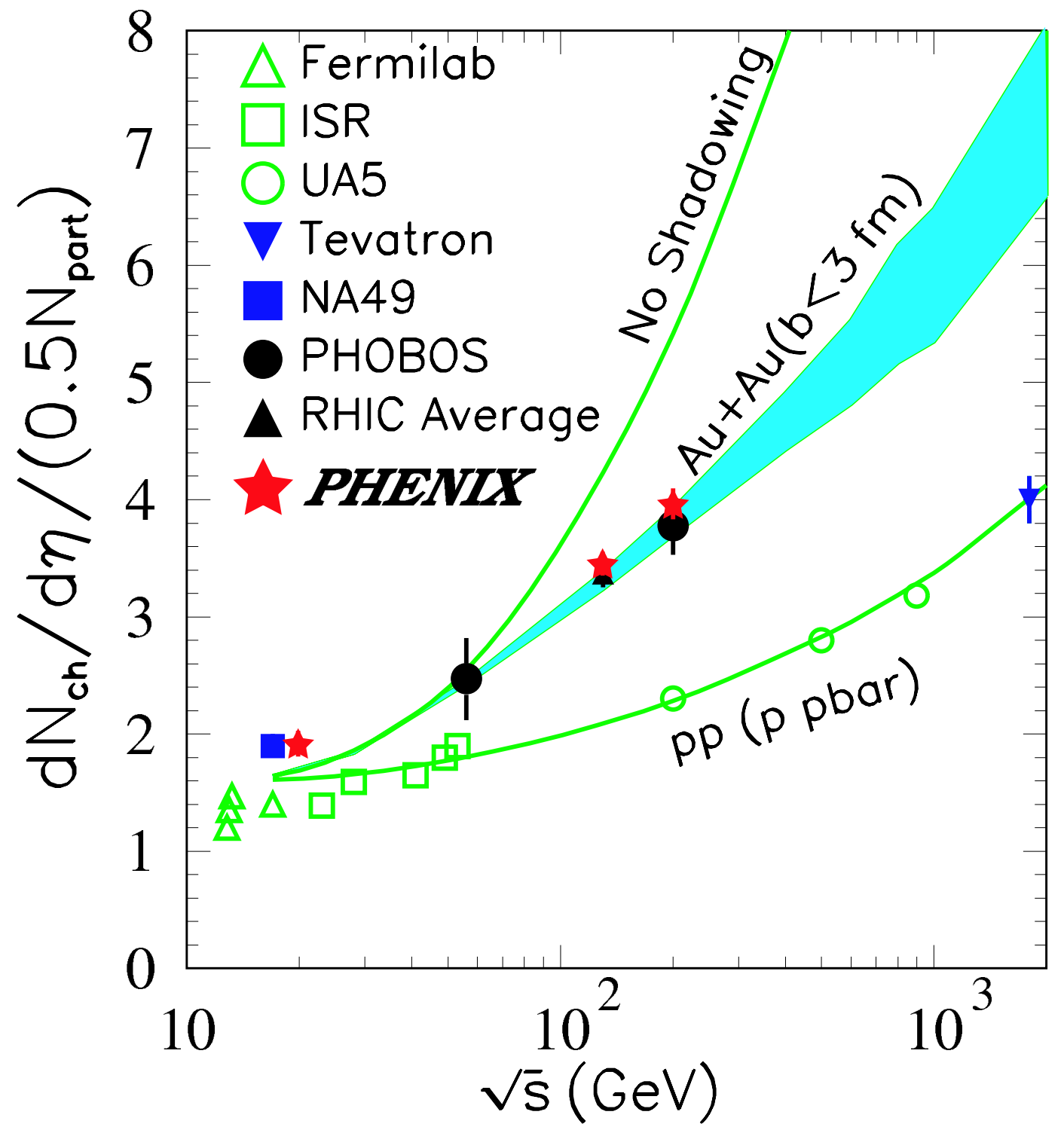

Fig. 7. Figure from Li and Wang [79] showing trends in final-state charged multiplicity per participant pair vs. (nucleon-nucleon) beam energy. (PHENIX data points[59] have been added.) The curves are the result of their two-component "hard/soft" model, which reproduces well the multiplicities from elementary $p(\bar{p})+p$ collisions at RHIC energies. The same model extended to nuclear collisions with no regulating mechanism on hard processes (the "No Shadowing" line) over-predicts the multiplicities in central RHIC collisions, while the data can be matched if substantial nuclear shadowing of gluons is invoked (shaded band).

collisions of two sheets of colored glass, with the produced quarks and gluons materializing at a time given by the inverse of the saturation momentum, $\tau=1 / Q_{s}$. Saturation of gluons with momenta below $Q_{s}$ provides a regulating mechanism that limits the rise in gluon - and later, hadron - multiplicity with centrality and beam energy. Models featuring this initial-state gluon saturation agree well with essentially all RHIC data on the multiplicity density, which is 


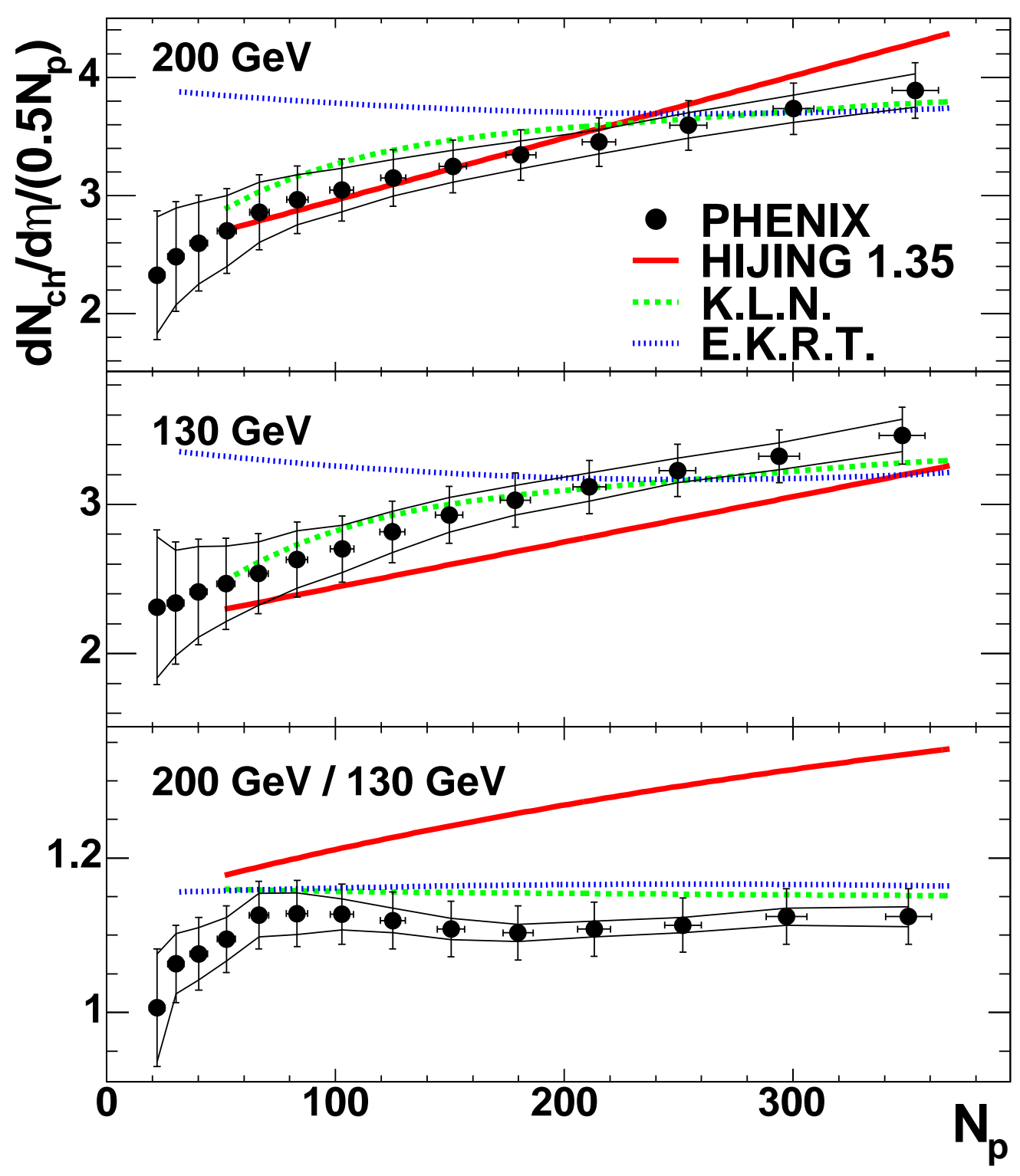

Fig. 8. Multiplicity per participant nucleon pair, as a function of centrality, for $\sqrt{s_{N N}}=130 \mathrm{GeV}$ and $200 \mathrm{GeV} \mathrm{Au}+\mathrm{Au}$ collisions as measured in PHENIX [59]; compared to theoretical predictions available in 2002. "HIJING" is a pQCD-based model [82], while "KLN" features gluon saturation in the initial state [83,84]; "EKRT" assumes saturation in the final state $[85,86]$.

dominated by low-momentum particles [83,84]. This is seen, for instance, in Fig. 8 .

In this picture, the total gluon multiplicity is proportional to $1 / \alpha_{s} \cdot Q_{s}^{2}$, which limits the number of low-momentum charged particles produced. $Q_{s}$ evolves slowly with collision centrality and beam energy. For central $\mathrm{Au}+\mathrm{Au}$ collisions, it has been estimated that the typical $m_{T}$ scale of the gluons "liberated" from the colored glass is about $1 \mathrm{GeV}$ per particle [77], which is above the lower limit 
of $0.53 \mathrm{GeV}$ per particle that we set above using the PHENIX data. Though there are fewer predictions of $E_{T}$ than total charged-particle production from gluon-saturation models, the existing models are broadly consistent with data at RHIC. Consequently, gluon saturation is considered to be a promising candidate for describing the initial state of RHIC collisions.

\subsection{Conclusions}

Using reasoning similar to that of Bjorken [74], combined with some simple formation-time arguments, we can draw the following conclusions from the PHENIX data on transverse energy production and overall particle multiplicity:

- The peak energy density in created secondary particles is at least $15 \mathrm{GeV} / \mathrm{fm}^{3}$, and this is most likely an underestimate. This is well in excess of the $\sim 1$ $\mathrm{GeV} / \mathrm{fm}^{3}$ required, according to lattice QCD predictions, to drive a $\mathrm{QCD}$ transition to QGP.

- We note that hydrodynamical calculations which reproduce the magnitude of elliptic flow observed at RHIC require local thermalization to occur very quickly, typically by $1 \mathrm{fm} / c$ or earlier (see Section 3.5). If the system does reach local equilibrium on this time scale then the energy density of the first thermalized state would be in excess of $5 \mathrm{GeV} / \mathrm{fm}^{3}$, well above the amount required to create the $\mathrm{QGP}$.

- Pre-RHIC expectations that $E_{T}$ and charged particle production would be dominated by factorized pQCD processes were contradicted by data, which showed only very modest increases with centrality and beam energy. A new class of models featuring initial-state gluon saturation compares well with RHIC multiplicity and $E_{T}$ data, and are also consistent with our Bjorkenstyle arguments for estimating energy densities at early times.

\section{THERMALIZATION}

A key question is whether the matter formed at RHIC is thermalized, and if so when in the collision was equilibration achieved. If thermalization is established early then evidence for strong transverse expansion can be potentially related to the equation of state of the dense matter produced at RHIC. To explore these issues we review several experimental observables from integral quantities (numbers of particles produced and in what ratios), to differen- 
tial distributions (measured $p_{T}$ and azimuthal distributions), to two-particle (HBT) correlations.

\subsection{Chemical Equilibrium}

For many years it has been known that the abundances of different hadron species in $e^{+}+e^{-}$and $p+\bar{p}$ reactions can be reproduced by statistical models $[87,88]$. This success is often attributed to hadronization statistically filling the available phase space. At RHIC there is also the possibility that the strong scattering deduced from the measurements of elliptic flow (section 3.3) may prove sufficient to establish chemical equilibrium.

The production of strange particles provides a means to check whether chemical equilibrium is achieved. For $e^{+}+e^{-}$and $p+\bar{p}$ reactions strange particle production is suppressed due to the small size of the system. This canonical suppression is largely removed for central heavy-ion collisions. If the measured strangeness yields are still lower than full equilibrium predictions, then the partial equilibrium can be quantified by a multiplicative factor of $\gamma_{s}$ for each strange quark in a hadron, where $\gamma_{s}=1$ for complete equilibration and $\gamma_{s}<1$ for partial equilibration.

Figure 9 shows the centrality dependence of $K / \pi$ and $p / \pi$ ratios in $\mathrm{Au}+\mathrm{Au}$ collisions at $\sqrt{s_{N N}}=200 \mathrm{GeV}[54]$. Both $K^{+} / \pi^{+}$and $K^{-} / \pi^{-}$increase rapidly for peripheral collisions, and then saturate or rise slowly from mid-central to the most central collisions. The ratios $p / \pi^{+}$and $\bar{p} / \pi^{-}$also increase from peripheral collisions but appear flatter than the $K / \pi$ ratios. Canonical statistical models [89] predict an increase in these ratios with centrality, as the larger system-size effectively places less of a constraint on conserved quantities. In addition the chemical parameters, $\mathrm{T}_{c h e m}$ and $\mu_{B}$, can also vary with centrality $[90,91]$.

Focusing on the ratios from central collisions at $\sqrt{s_{N N}}=200 \mathrm{GeV}$, the data are compared to the thermal model data analysis of Kaneta and $\mathrm{Xu}$ [90] in Fig. 10. The extracted thermal parameters from this fit are $\mathrm{T}_{\text {chem }}=157 \pm 3$ $\mathrm{MeV}, \mu_{B}=23 \pm 3 \mathrm{MeV}$, and $\gamma_{s}=1.03 \pm 0.04$. A large $\gamma_{s}$ is also found by STAR [92] who extract $\gamma_{s}=0.96 \pm 0.06$, while Cleymans et al. [91] extract $\gamma_{s}$ that increases from $\gamma_{s} \simeq 0.85$ in peripheral collisions to $\gamma_{s} \simeq 0.95$ for central collisions at RHIC. Similar fits to the central RHIC data are obtained by Braun-Munzinger et al. [93] who assume complete chemical equilibration, i.e. $\gamma_{s}=1$

We note that there are differences in the temperature parameter extracted by the different authors. Kaneta and $\mathrm{Xu}[90]$ extract $\mathrm{T}_{\text {chem }}=157 \pm 3 \mathrm{MeV}$ which is lower than that extracted by both Braun-Munzinger et al. [89] of $\mathrm{T}_{\text {chem }}=$ 

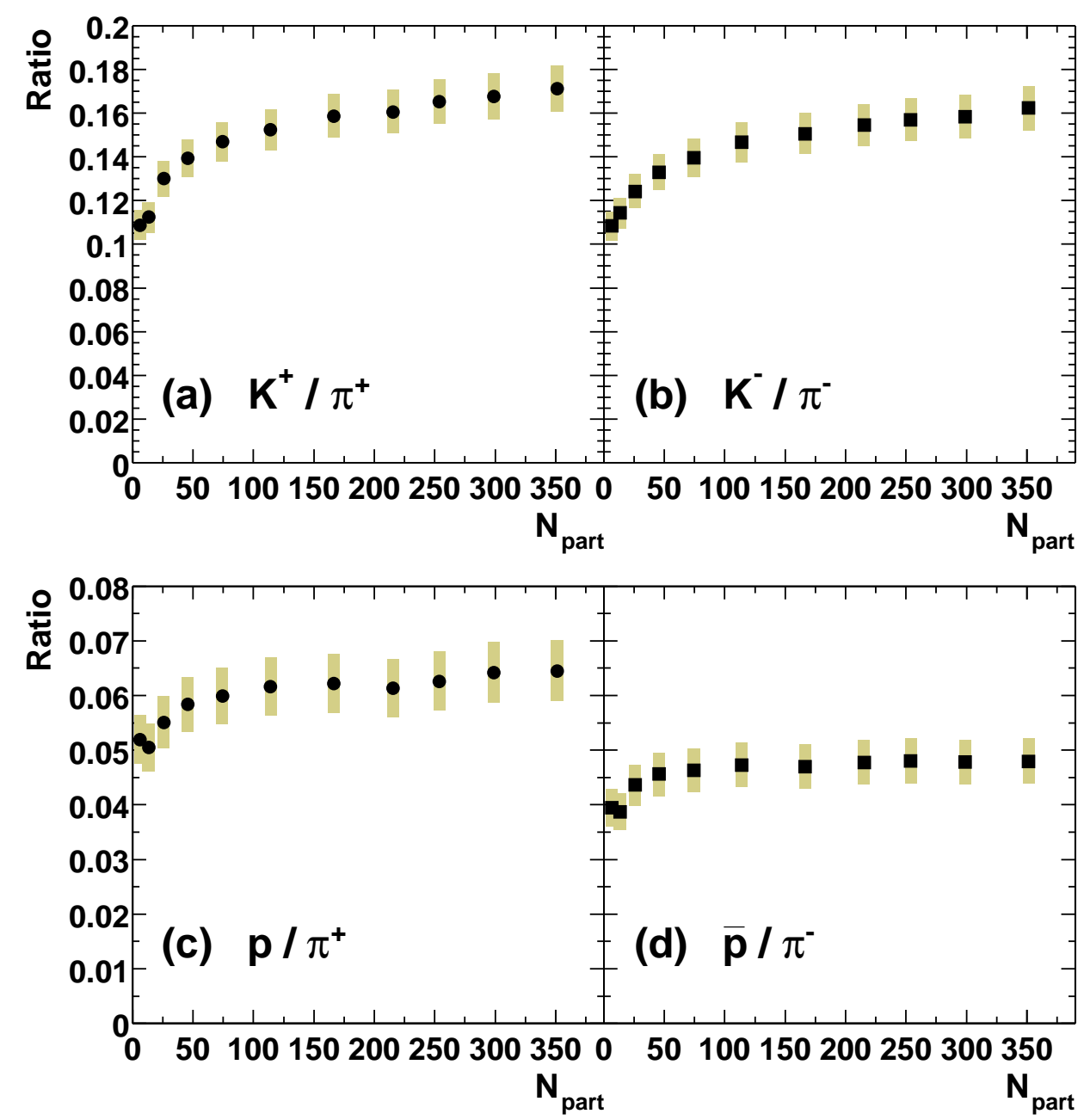

Fig. 9. Centrality dependence of particle ratios for (a) $K^{+} / \pi^{+}$, (b) $K^{-} / \pi^{-}$, (c) $p / \pi^{+}$, and (d) $\bar{p} / \pi^{-}$in $\mathrm{Au}+\mathrm{Au}$ collisions at $\sqrt{s_{N N}}=200 \mathrm{GeV}$ [54].

$177 \pm 7 \mathrm{MeV}$ and Cleymans al. [91] of $\mathrm{T}_{\text {chem }}=165 \pm 7 \mathrm{MeV}$. However, both Braun-Munzinger et al. [89] and Magestro [94] discuss the sensitivity of the extracted temperature to corrections from feed-down from decays. Cleymans et al. [91] estimate that over $70 \%$ of $\pi^{+}$in the thermal model fits come from the decay of resonances.

At lower beam energies there is controversy over whether strangeness is in full chemical equilibrium. Becattini et al. [95] use data that is integrated over the full rapidity and find that strangeness is in partial equilibrium, i.e. at the AGS $\gamma_{s}=0.65 \pm 0.07$ and at the SPS $\gamma_{s}=0.84 \pm 0.03$. Braun-Munzinger et al. [89] instead use ratios measured at mid-rapidity which typically have larger strange/no-nstrange values, and, hence, they obtain acceptable fits with $\gamma_{s}=1$ at both AGS and SPS energies. At RHIC energies thermal model comparisons all use mid-rapidity data; a choice that is motivated in part by the separation between fragmentation regions and central particle production. 


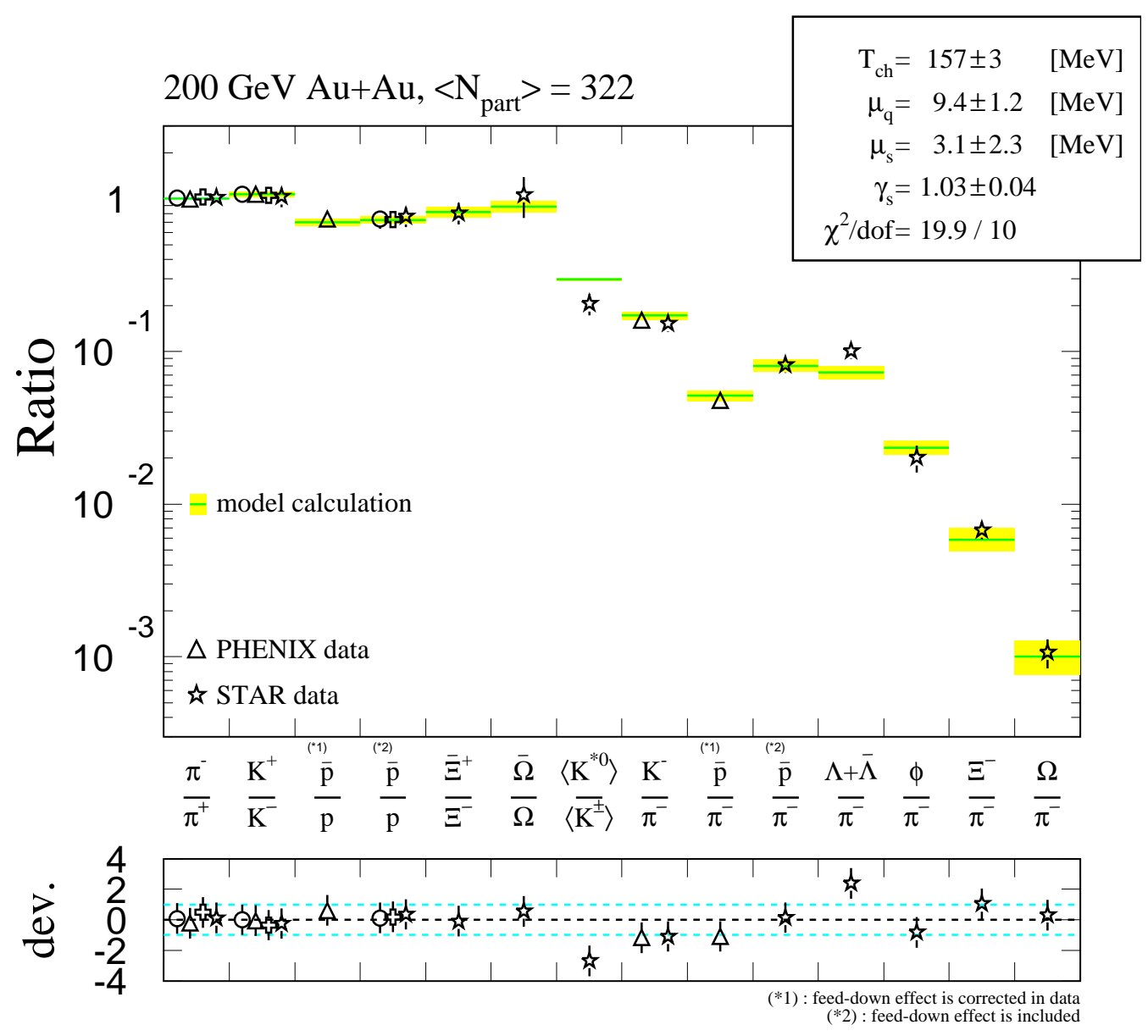

Fig. 10. Comparison of PHENIX (triangles), STAR (stars), BRAHMS (circles), and PHOBOS (crosses) particle ratios from central $\mathrm{Au}+\mathrm{Au}$ collisions at $\sqrt{s_{N N}}=200$ $\mathrm{GeV}$ at mid-rapidity. The thermal model descriptions from Kaneta [90] are also shown as lines. See Kaneta [90] for the experimental references.

In contrast to the controversies at lower beam energies, the observation that strangeness is equilibrated is common to all thermal calculations that reproduce RHIC data. This is consistent with chemical equilibrium being obtained before hadronization, though does not prove that this is the case. An alternative explanation is that scattering in the hadronic phase could increase $\gamma_{s}$ to 1 , though small interaction cross sections imply that it may be difficult to equilibrate the multi-strange baryons before the hadrons freezeout.

\subsection{Spectra}

Hadron spectra reflect conditions late in the reaction, as well as the integrated effects of expansion from the beginning of the collision. Figure 11 shows the $p_{T}$ distributions for pions, kaons, protons, and anti-protons in both central (top 
panel) and peripheral collisions (bottom panel) [54]. The pion spectra have a

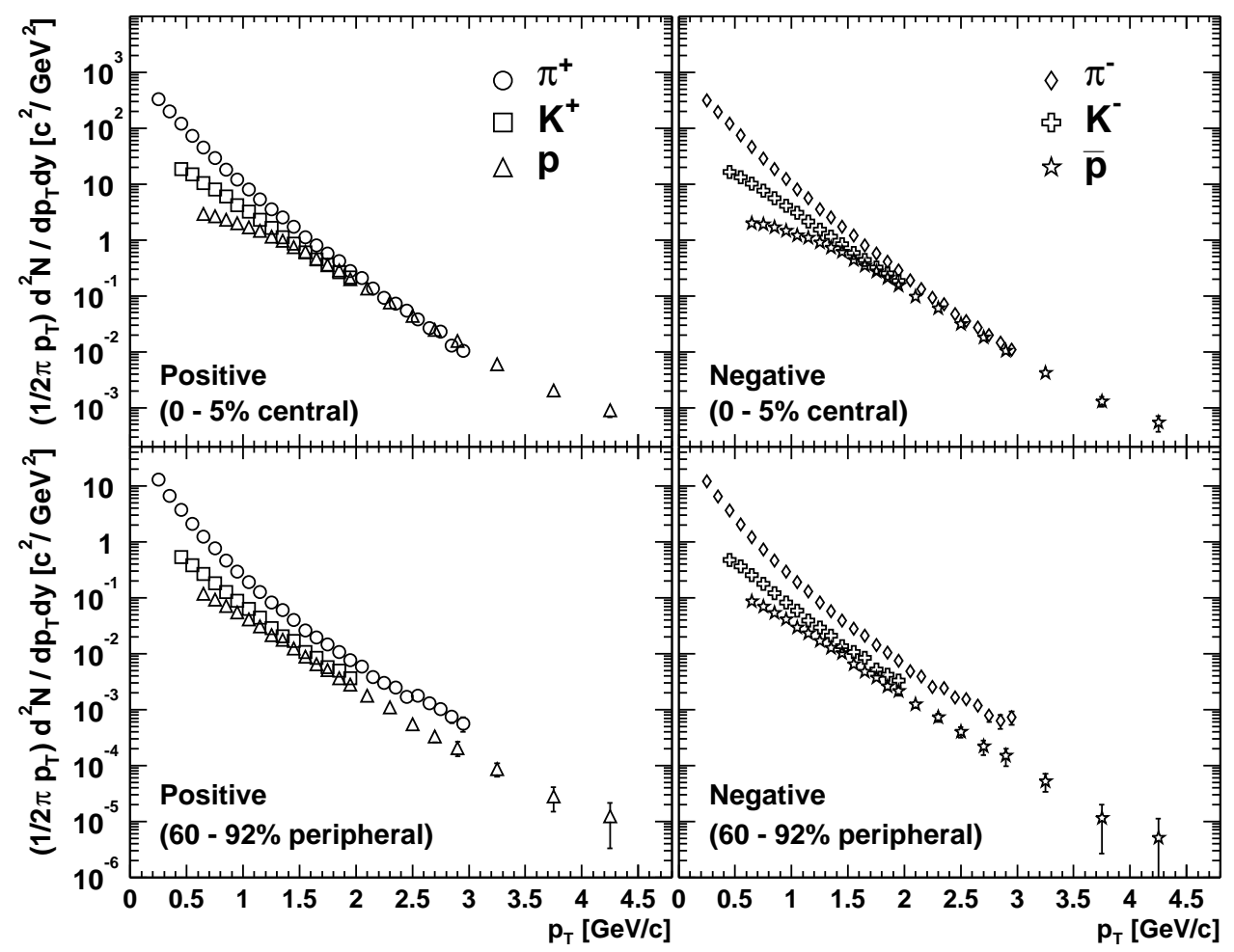

Fig. 11. Transverse momentum distributions for pions, kaons, protons, and anti-protons in $\mathrm{Au}+\mathrm{Au}$ collisions at $\sqrt{s_{N N}}=200 \mathrm{GeV}[54]$.

concave shape at low $\mathrm{p}_{T}$ where many of the pions may come from the decay of resonances: $\Delta, \rho$ etc. The kaon spectra are approximately exponential over the full measured $\mathrm{p}_{T}$ range, whereas the proton spectra flatten at low $\mathrm{p}_{T}$ for the most central collisions. A striking feature is that the proton and anti-proton spectra in central collisions become comparable in yield to the pion spectra above $2 \mathrm{GeV} / c$. This is more fully discussed in Section 7 .

One way to characterize the change in spectra as a function of centrality is to calculate $\left\langle p_{T}\right\rangle$ for each spectrum [54] as shown in Fig. 12. The $\left\langle p_{T}\right\rangle$ increases for all particles as a function of centrality with the largest change occurring in peripheral collisions $\left(N_{\text {part }}<100\right)$. Across the different particles the increase is largest for protons and anti-protons. This is consistent with a collective expansion velocity that increases with centrality to produce the largest increase in $\left\langle p_{T}\right\rangle$ for the heaviest particles.

The pion, kaon, and proton spectra can all be fit using an ansatz of a thermal, expanding source $[96,48]$ to extract the collective transverse expansion velocity $\left\langle\beta_{T}\right\rangle$ as well as the temperature at freezeout, $\mathrm{T}_{\text {fo }}$. Figure 13 shows $\left\langle\beta_{T}\right\rangle \sim 0.45$ at AGS energies $[97,98]$, which increases to $\left\langle\beta_{T}\right\rangle \sim 0.5$ at the SPS $[99,100,101]$ 


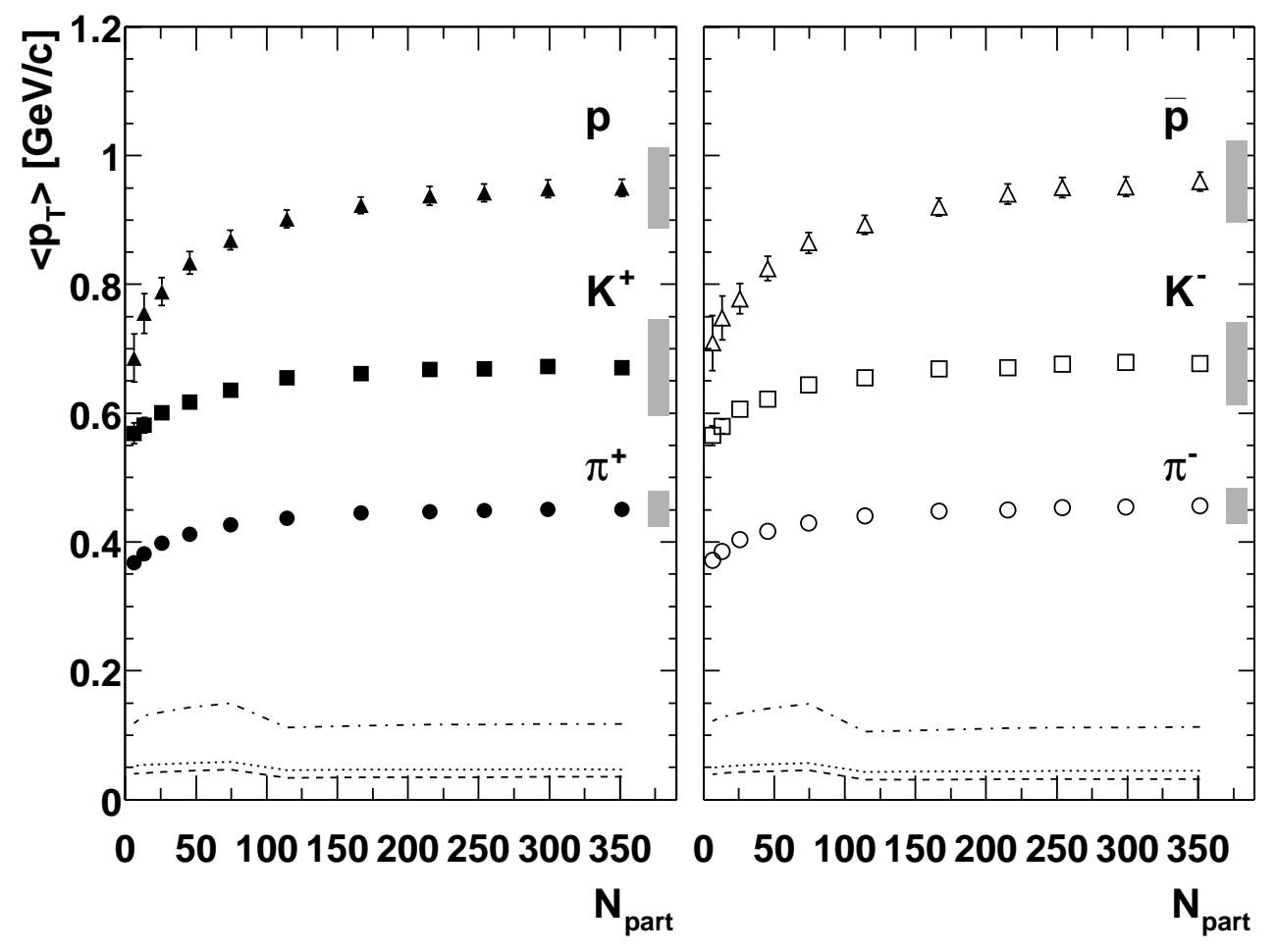

Fig. 12. Mean transverse momentum as a function of $N_{\text {part }}$ for pions, kaons, protons and anti-protons in $\mathrm{Au}+\mathrm{Au}$ collisions at $\sqrt{s_{N N}}=200 \mathrm{GeV}$ [54]. The systematic errors from extrapolation, which are scaled by a factor of two for clarity, are shown in the bottom for protons and anti-protons (dashed-dotted lines), kaons (dotted lines), and pions (dashed lines). The shaded bars to the right represent the systematic error.

and RHIC [48,102]. All the above fits use similar model assumptions of a linear velocity profile and a Woods-Saxon density profile. That the spectra at these beam energies can be reproduced by a thermal source is necessary, but not sufficient, evidence for thermal equilibrium at each of these energies. However, it is difficult to draw strong conclusions from the increase in $\left\langle\beta_{T}\right\rangle$ as a function of beam energy since the parameters $\left\langle\beta_{T}\right\rangle$ and $\mathrm{T}_{f o}$ are strongly anticorrelated and their values depend on fit ranges and treatment of decays.

\subsection{Elliptic Flow}

At the beginning of a heavy ion collision, the spatial distribution of the colliding matter resembles an ellipsoid due to the incomplete overlap of the two colliding nuclei. Any strong scattering in this early stage converts the spatial anisotropy to a momentum anisotropy which is observable as an elliptic flow of the emitted hadrons. Elliptic flow is a self-limiting phenomenon, which is readily understood in the thermodynamic limit. If strong scattering is sufficient to establish local thermal equilibrium, then the pressure gradient is largest in 


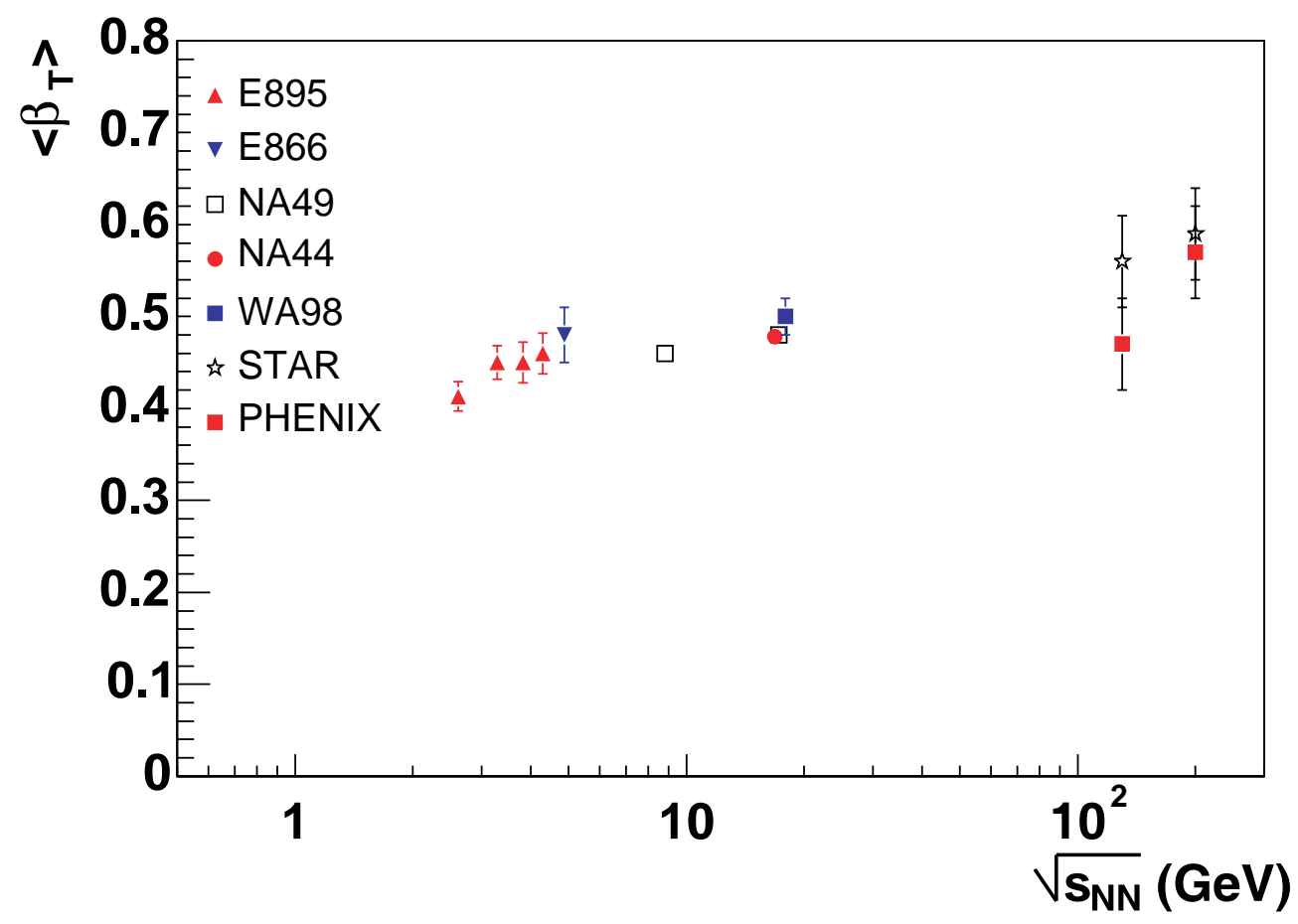

Fig. 13. Beam-energy dependence of the extracted mean transverse expansion velocity as a function of beam energy from simultaneous fits to spectra of different mass $[97,98,99,100,101,48,102]$.

the shortest direction of the ellipsoid. This gradient produces higher momenta in that direction, quickly reducing the spatial asymmetry.

The absence of any strong scattering in the early stage of the colliison would reduce the amount of elliptic flow that could be created. If the initially produced particles are allowed to initially free stream and reach local equilibrium only after some time delay, then the spatial anisotropy at the start of hydrodynamic evolution will be reduced; the longer the delay, the greater the reduction. Following the prescription of Kolb et al. [78], we plot in Fig. 14 the eccentricity after a time delay $\Delta t$ compared to its value at formation time, as a function of $\mathrm{Au}+\mathrm{Au}$ collision centrality. The eccentricity $(\varepsilon)$ of the reaction zone is

$$
\varepsilon=\frac{\left\langle y^{2}\right\rangle-\left\langle x^{2}\right\rangle}{\left\langle y^{2}\right\rangle+\left\langle x^{2}\right\rangle}
$$

The eccentricity can be analytically calculated once the density profile of the nuclei is chosen (typically a Woods-Saxon shape). It can also be calculated using Monte Carlo techniques, where the positions of those nucleons that participate in the reaction are used to calculate the averages in Eq. 7. From Fig. 14 we can see that for time delays of $2 \mathrm{fm} / c$ or greater the magnitude of the eccentricity is significantly reduced, and its shape vs. centrality is also 
altered.

If locally equilibrated hydrodynamics is taken as the mechanism for generating elliptic flow, then the observation of any substantial amount of elliptic flow can be taken as evidence that local thermal equilibrium is achieved on a time scale before the spatial anisotropy would be completely erased. The order of this time-scale would be $t \sim R / c$, where $R$ is the nuclear radius. However, the hydrodynamical calculations we will examine here (see Sec. 3.5 and Table 1) all require quite short thermalization times, from $0.6-1.0 \mathrm{fm} / c$, in order to reproduce the magnitude of elliptic flow observed at RHIC.

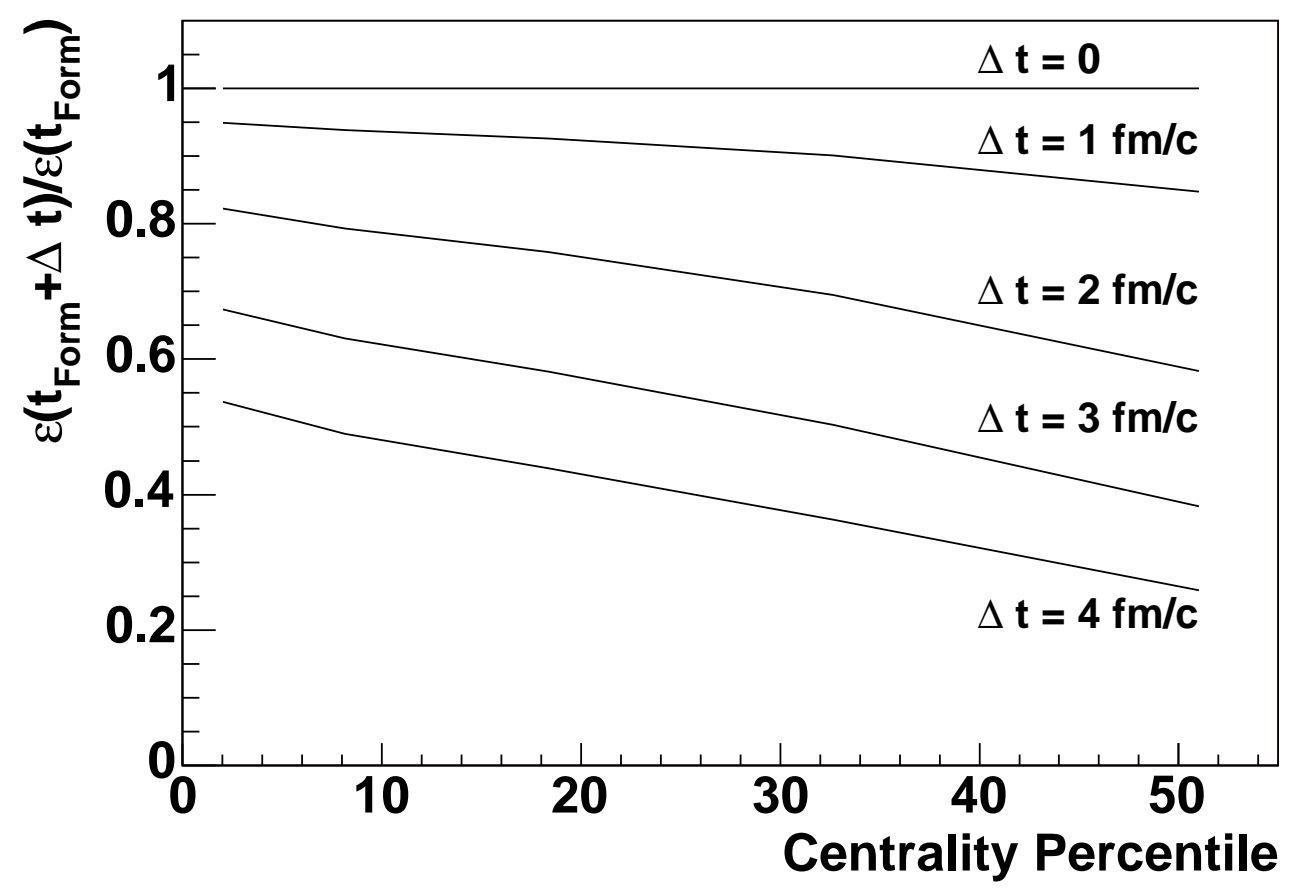

Fig. 14. The ratio of the eccentricity after a time delay $\Delta t$ compared to its value at formation time, as a function of $\mathrm{Au}+\mathrm{Au}$ collision centrality. The calculations follow the prescription of [78] where the produced particles are allowed to free-stream at first and reach local equilibrium only after some time delay.

The azimuthal anisotropy of the spectra can be characterized in terms of Fourier coefficients, which at RHIC are dominated by the elliptic flow, the second Fourier coefficient, $v_{2}\left(p_{T}\right)$, where

$$
\frac{d^{2} N}{d \phi d p_{T}}=N_{0}\left(1+2 v_{2}\left(p_{T}\right) \cos (2 \phi)\right)
$$

Both the first Fourier coefficient, $v_{1}$, and higher order coefficients have been neglected in the above expression. 
The most direct evidence that $v_{2}$ is related to spatial asymmetries present early in the reaction is that $v_{2}$ at low $p_{T}$ approximately scales with the initial eccentricity $(\varepsilon)$ of the reaction zone. The measured values of $v_{2}$ normalized by $\varepsilon$ are shown in Fig. 15 vs. centrality for two different $p_{T}$ ranges [46]. At low momentum $v_{2} / \varepsilon$ is independent of centrality to within $20 \%$. This scaling is increasingly broken at higher $p_{T}$.

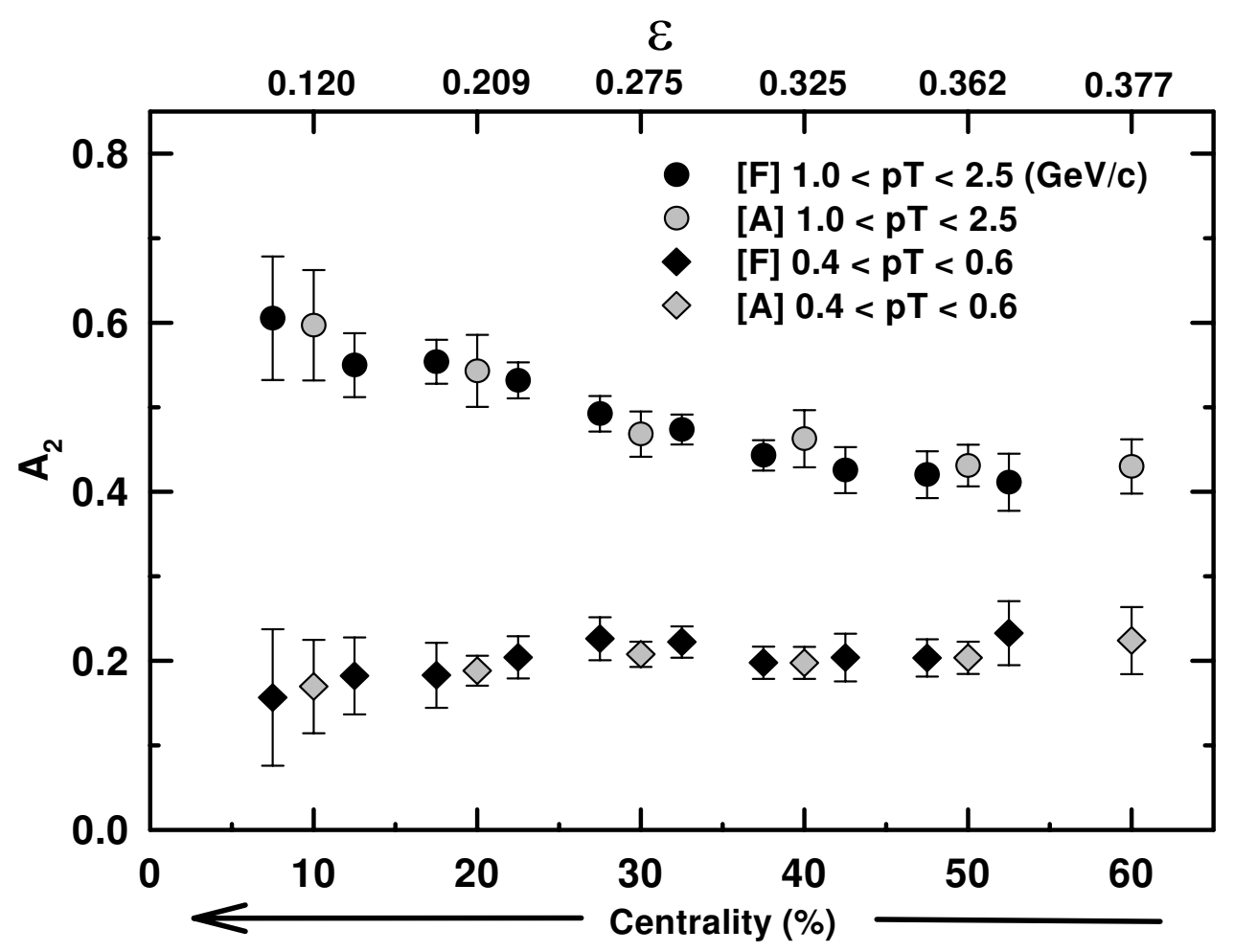

Fig. 15. $A_{2}=v_{2} / \varepsilon$ vs. centrality for $\mathrm{Au}+\mathrm{Au}$ collisions at $\sqrt{s_{N N}}=130 \mathrm{GeV}$ [46]. The data points come from two different types of two-particle correlations: "fixed" $p_{T}$ correlations when both particles are at the same $p_{T}$ (points are labeled as " $\mathrm{F}$ "), and "assorted" $p_{T}$ correlations when the two particles have different $p_{T}$ (points are labeled as "A"). In this case the labeled $p_{T}$ range is for the higher-momentum particle of the pair.

The measured values of the integrated $v_{2}$ at RHIC are larger than those at lower energies, but this is in part due to the fact that $v_{2}\left(p_{T}\right)$ increases with $p_{T}$ and $\left\langle p_{T}\right\rangle$ increases as a function of beam energy. To remove this effect we will concentrate on the differential flow, i.e. the shape of $v_{2}\left(p_{T}\right)$.

To make a uniform comparison between different colliding nuclei $(\mathrm{Pb}+\mathrm{Pb}$ at SPS and $\mathrm{Au}+\mathrm{Au}$ at $\mathrm{RHIC}$ ) as well as different impact parameter selections from the different experiments, we normalize $v_{2}$ by the eccentricity, $\varepsilon$, as shown in Fig. 16. The values of $\varepsilon$ have been calculated via a Glauber Monte Carlo using Woods-Saxon density distributions for the $\mathrm{Au}$ and $\mathrm{Pb}$ nuclei. The aver- 


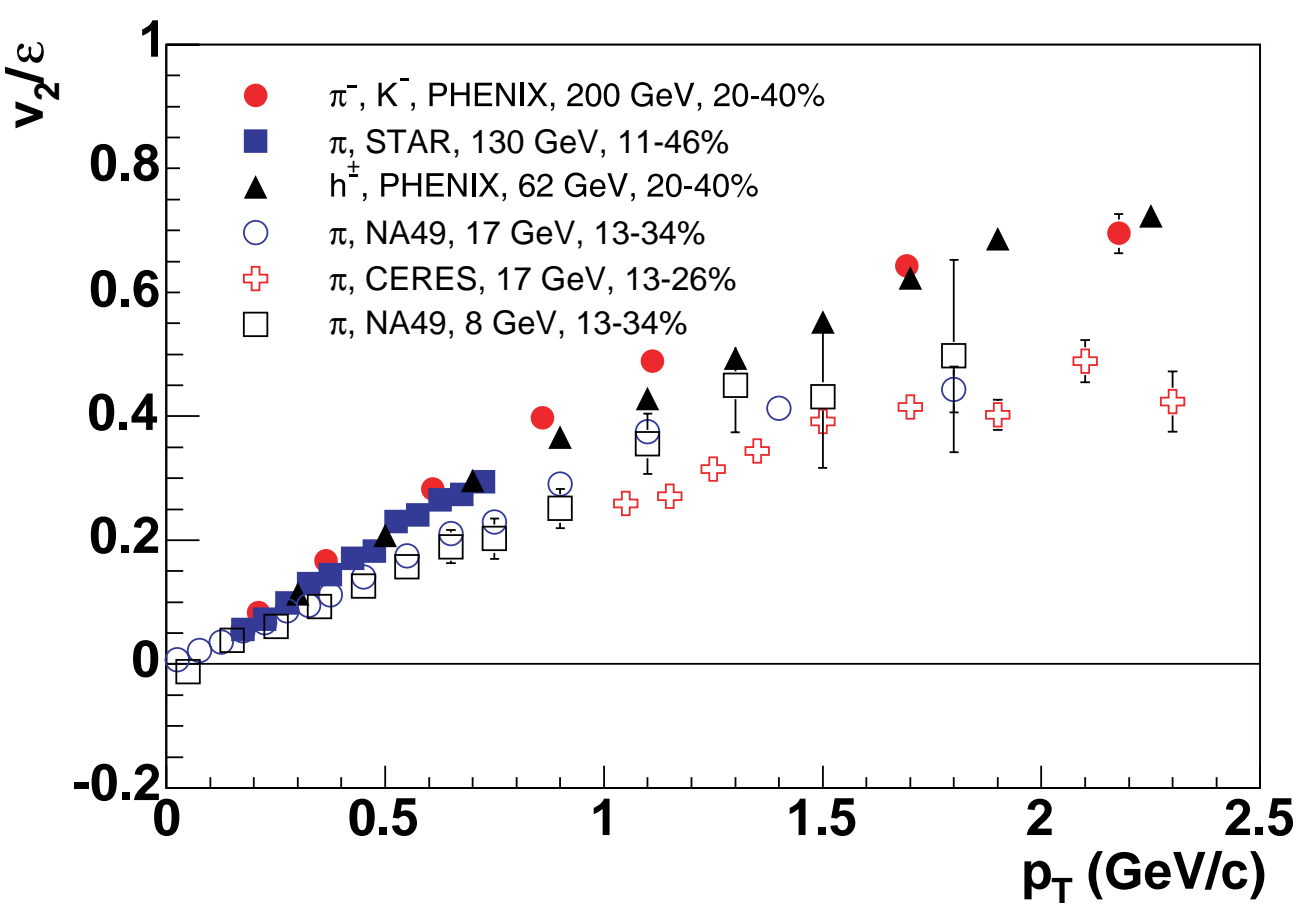

Fig. 16. $v_{2}\left(p_{T}\right) / \varepsilon$ vs. $p_{T}$ for mid-central collisions at RHIC (filled symbols) and SPS (open symbols). Dividing by eccentricity removes to first order the effect of different centrality selections across the experiments [50,103,70,104,105].

ages in Eq. 7 are over the participating nucleons, hence $\varepsilon$ is calculated at the start of the collision. The pion data in Fig. 16 show that $v_{2}\left(p_{T}\right) / \varepsilon$ increases approximately linearly with $p_{T}$ for low $p_{T}$. The rate of increase of $v_{2} / \varepsilon$ as a function of $p_{T}$ is larger at RHIC $[50,103]$ than at SPS [104,105] as can most easily be seen by calculating the slope of $v_{2} / \varepsilon$ below $p_{T}=1 \mathrm{GeV} / c$ (Fig. 17). The slope $\left(d v_{2} / d p_{T}\right) / \varepsilon$ increases from SPS to RHIC by approximately $50 \%$. Hydrodynamical calculations [106] shown in Fig. 17 reproduce the data both at RHIC and at CERN SPS within one standard deviation. More extensive comparisons with hydro calculations will be discussed in section 3.5, while the behavior of $v_{2}$ at higher $p_{T}$, which follows a scaling with respect to the number of quarks, is discussed in Section 7.

Further insight into the expansion dynamics can be obtained from the mass dependence of $v_{2}\left(p_{T}\right)$ shown in Fig. 18 for pions, kaons and protons [50] along with a comparison with an early hydrodynamic model calculation [107]. The $v_{2}\left(p_{T}\right)$ for pions is larger than for kaons and protons at low $p_{T}$, and this mass ordering has been explained as resulting from radial expansion[107] that produces a larger distortion of the elliplic flow induced velocity profile for larger hadron masses. However, as will be discussed in Section 3.5, this calculation fails to reproduce the proton spectra, and attempts to remedy this failure lead to calculations that no longer reproduce the measured $v_{2}$ for pions and protons. 


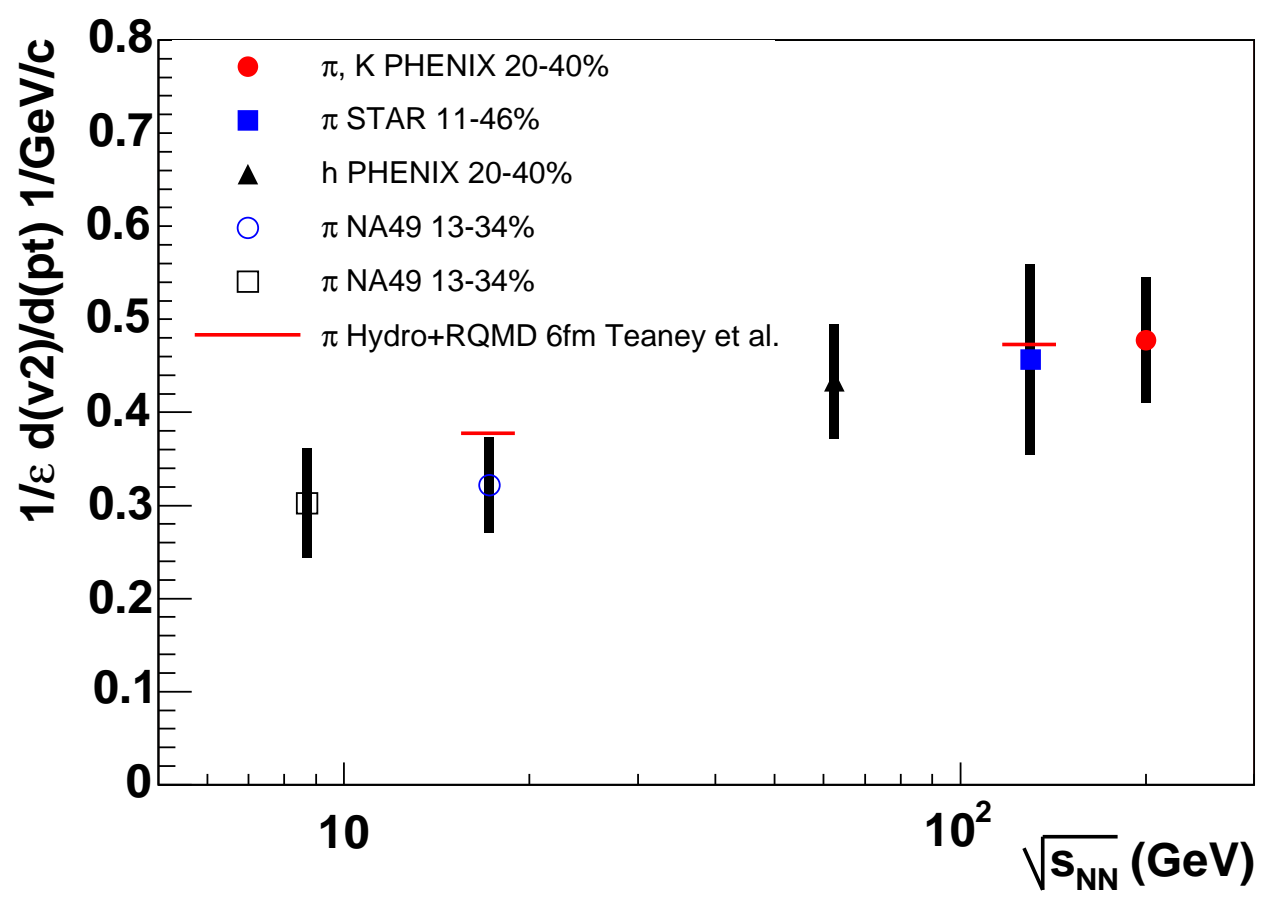

Fig. 17. The slope of the scaled elliptic flow, $\left(d v_{2} / d p_{T}\right) / \varepsilon$, for mid-central collisions at RHIC (filled symbols) and the SPS (open symbols). The slope is calculated from the data in Fig. 16 for the data $p_{T}<1 \mathrm{GeV} / c$. The solid error bars represent the total systematic error including the systematic error on $v_{2}$ and $\varepsilon[50,103,70,104]$.

\section{$3.4 H B T$}

Bose-Einstein correlations between identical particles provide a measure of the space-time extent of the source at the end of the reaction. Because the extracted source parameters as measured by the HBT technique are driven by space-time correlations, HBT results are sensitive to expansion dynamics integrated throughout the collision. HBT measurements were originally motivated by theoretical predictions of a large source size and/or a long duration of particle emission $[108,109,110]$ — which would result from the presence of a long-lived mixture of phases in the matter as it undergoes a first-order phase transition from a quark-gluon plasma back to the hadronic phase.

In HBT analyses, multidimensional Gaussian fits are made to the normalized relative momentum distributions yielding fit parameters, $R_{\text {long }}, R_{\text {side }}, R_{\text {out }}$ [111], also referred to as HBT radii, where

$$
C_{2}=1+\lambda \exp \left(-R_{\text {side }}^{2} q_{\text {side }}^{2}-R_{\text {out }}^{2} q_{\text {out }}^{2}-R_{\text {long }}^{2} q_{\text {long }}^{2}\right)
$$

The coordinate system is chosen so that the longitudinal direction is parallel to the beam axis, the out direction is in the direction of the pair's total transverse 


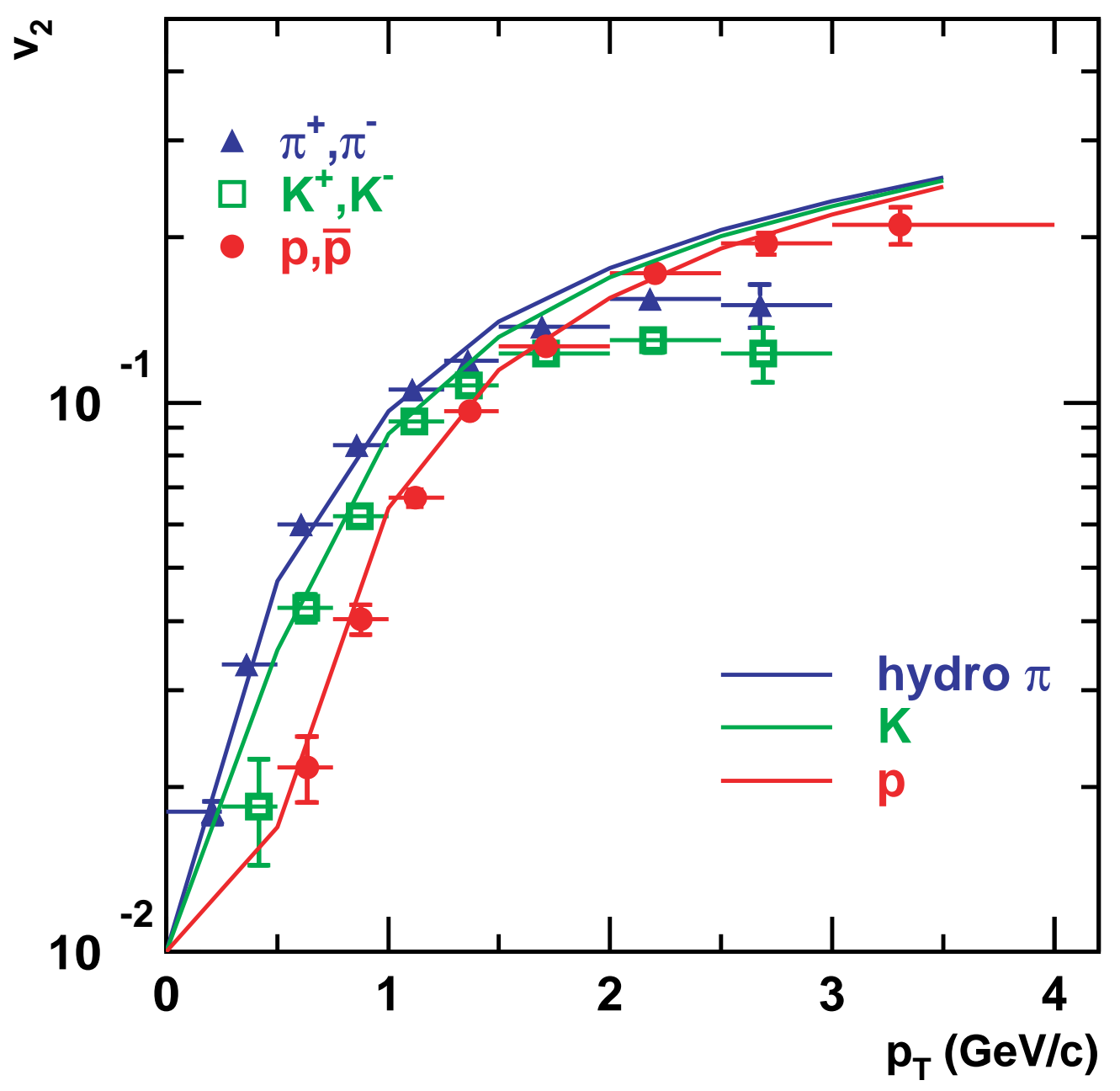

Fig. 18. $v_{2}\left(p_{T}\right)$ for pions, kaons and protons produced in minimum-bias collisions at RHIC [50] compared to hydro calculations from Huovinen et al. [107].

momentum, and the side direction is in the transverse plane perpendicular to the out axis. For dynamic (i.e. expanding) sources, the HBT radii depend on the mean transverse momentum of the particle pairs, $k_{T}=\left|\mathbf{p}_{1 \mathrm{~T}}+\mathbf{p}_{2 \mathrm{~T}}\right| / 2$, and correspond to lengths of homogeneity: regions of the source which emit particles of similar momentum [112]. Measuring the $k_{T}$ dependence of HBT radii provides essential constraints on dynamical models [113]. In particular, the ratio $R_{\text {out }} / R_{\text {side }}$ is predicted to be larger than unity for sources which emit particles over a long time.

The measured $k_{T}$ dependence of all radii [56] and the ratio $R_{\text {out }} / R_{\text {side }}$ are shown in Fig. 19, along with STAR results [114]. The data from PHENIX and STAR are in excellent agreement. Both sets of data have been corrected for Coulomb repulsion between the detected particles.

The measured radii all decrease with increasing $k_{T}$ as expected for a rapidly 

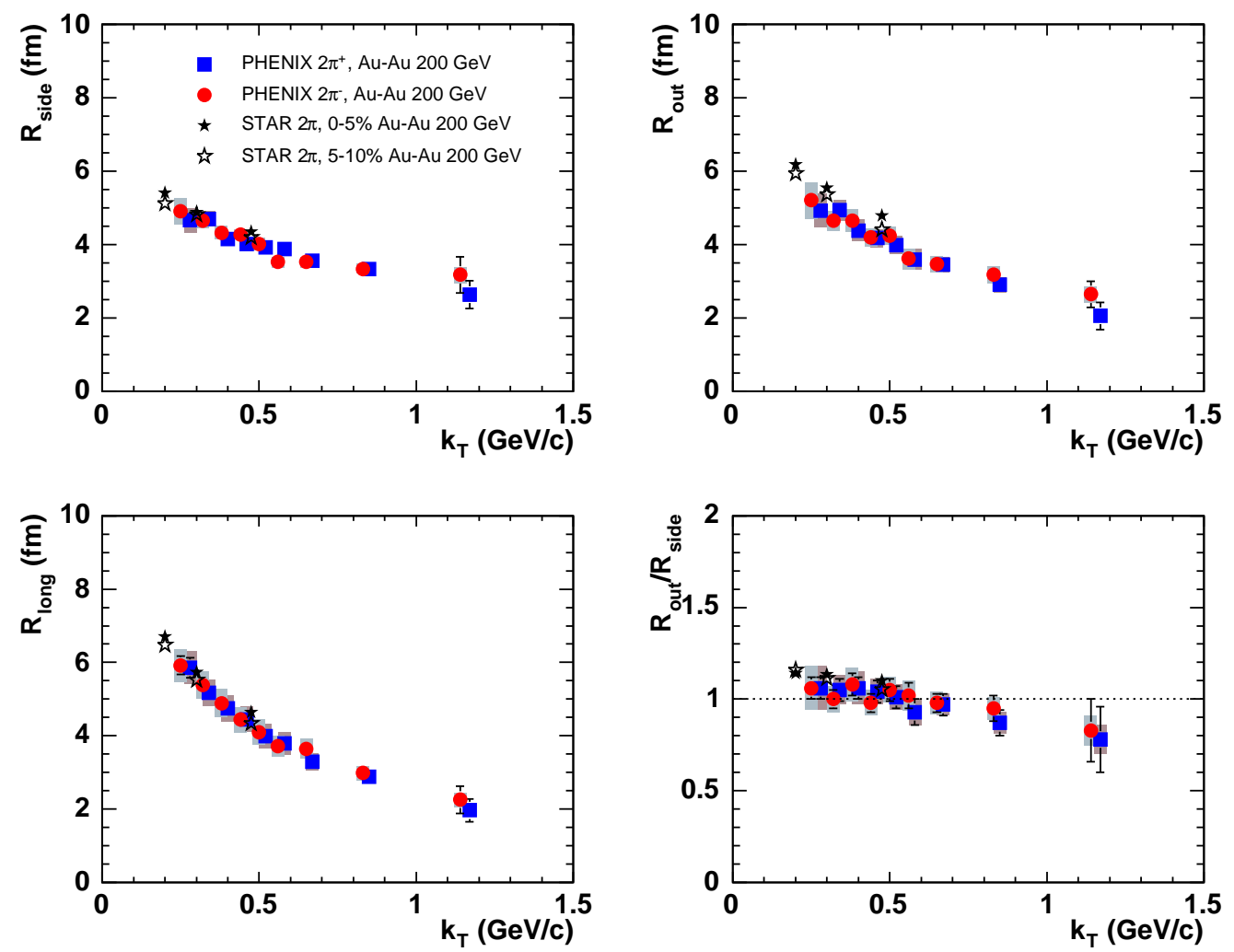

Fig. 19. The $k_{T}$ dependence of the Bertsch-Pratt parameters for $\pi^{+} \pi^{+}$(blue square) and $\pi^{-} \pi^{-}$(red circle) for $0-30 \%$ centrality with statistical error bars and systematic error bands. Results from PHENIX [56] and STAR [114] are overlaid.

expanding source. The ratio $R_{\text {out }} / R_{\text {side }}$ was measured to be 1 within errors, with a slight systematic decrease for increasing $k_{T}$. As is discussed in the next section, these data have excluded the validity of a large majority of hydrodynamical models developed to describe $\mathrm{Au}+\mathrm{Au}$ collisions at $\mathrm{RHIC}$, indicating that in their present form these models do not describe well the space-time evolution of the $\mathrm{Au}+\mathrm{Au}$ collisions.

\subsection{Hydrodynamic Model Comparisons}

Many of the experimental features in the spectra and elliptic flow are consistent with equilibrium being established early in the collision with large pressure gradients that drive a strong expansion. Moving from a statement of "consistency" to a statement that equilibrium has been "established" is difficult. Some progress can be made by comparing the data to hydrodynamic models that assume full equilibrium early in the collision.

A variety of hydrodynamic models have been published. Our approach is to confront these models with the following broad set of data; $v_{2}\left(p_{T}\right)$, spectra, 
and HBT. In this paper we will not compare the data with hydro-inspired parameterized fits, e.g. blast-wave [115] or Buda-Lund [116] models, but will restrict ourselves to dynamical hydro models.

In Figs. 20 and 21, hydro calculations that include a phase transition from the QGP phase to a hadronic phase are shown with solid lines, while hydro calculations that do not include a pure QGP phase at any stage in the dynamics are drawn with dashed lines. The four calculations that include a QGP phase all assume an ideal gas EOS for the QGP phase, a resonance gas for the hadronic phase and connect the two using a first-order phase transition and a Maxwell construction. These calculations use latent heats that range from $0.8 \mathrm{GeV} / \mathrm{fm}^{3}$ (Teaney et al. [106]) to $1.15 \mathrm{GeV} / \mathrm{fm}^{3}$ (Huovinen et al. [107] and Kolb et al. [117]), to $1.7 \mathrm{GeV} / \mathrm{fm}^{3}$ (Hirano et al. $[118,119]$ ). For comparison the bag model of the nucleon with external bag pressure $B=(230 \mathrm{MeV})^{4}$ and a $T_{\text {crit }}=164 \mathrm{MeV}$ produces a latent heat of $1.15 \mathrm{GeV} / \mathrm{fm}^{3}$ [120]. The calculations that do not include a QGP phase (dashed lines) either include a hadron phase and a phase mixture by forcing the latent heat of the transition to infinity [106], or use an hadronic resonance gas equation of state, i.e. no mixed or QGP phases [107].

The calculations also differ in how they solve the hydro equations and how they treat the final hadronic phase. The work of Hirano, Tsuda, and Nara cited here are the only calculations in this paper that solve the hydro equations in $3 \mathrm{D}$ $[118,119]$. For the final hadronic stage Teaney [106] uses a hybrid model that couples the hadronic phase to RQMD to allow hadrons to freezeout according to their cross section, i.e. for chemical equilibrium to be broken in the hadronic phase. Hirano [118] and Kolb [120] both allow for partial chemical equilibrium by chemically freezing out earlier than the kinetic freezeout. This has been done in order to reproduce the large proton yield measured at RHIC (see later in this section). In contrast, Huovinen [107] maintains full chemical equilibrium throughout the hadronic phase.

Figure 20 compares these calculations to the measured minimun-bias proton and pion $v_{2}\left(p_{T}\right) / \varepsilon$. Minimim-bias results were chosen in order to have the broadest set of data and model calculations for comparison. The four calculations that include a phase transition from the QGP phase to a hadronic phase (solid lines) reproduce the low- $p_{T}$ proton data better than the two hydro calculations that do not have a QGP phase at any stage in the dynamics (dashed lines). The presence of the first-order QGP phase transition softens the EOS which reduces the elliptic flow. At higher $p_{T}$ there is considerable variation between the models. Part of this is due to how the final hadronic stage is modeled. For example, Kolb's (solid light-blue line) and Hirano's (solid darkblue line) calculations allow for partial chemical equilibrium in the final stage compared to Huovinen (solid green line) which chemically freezes out late in the collision. The difference is observable above $p_{T} \sim 1 \mathrm{GeV} / c$. 

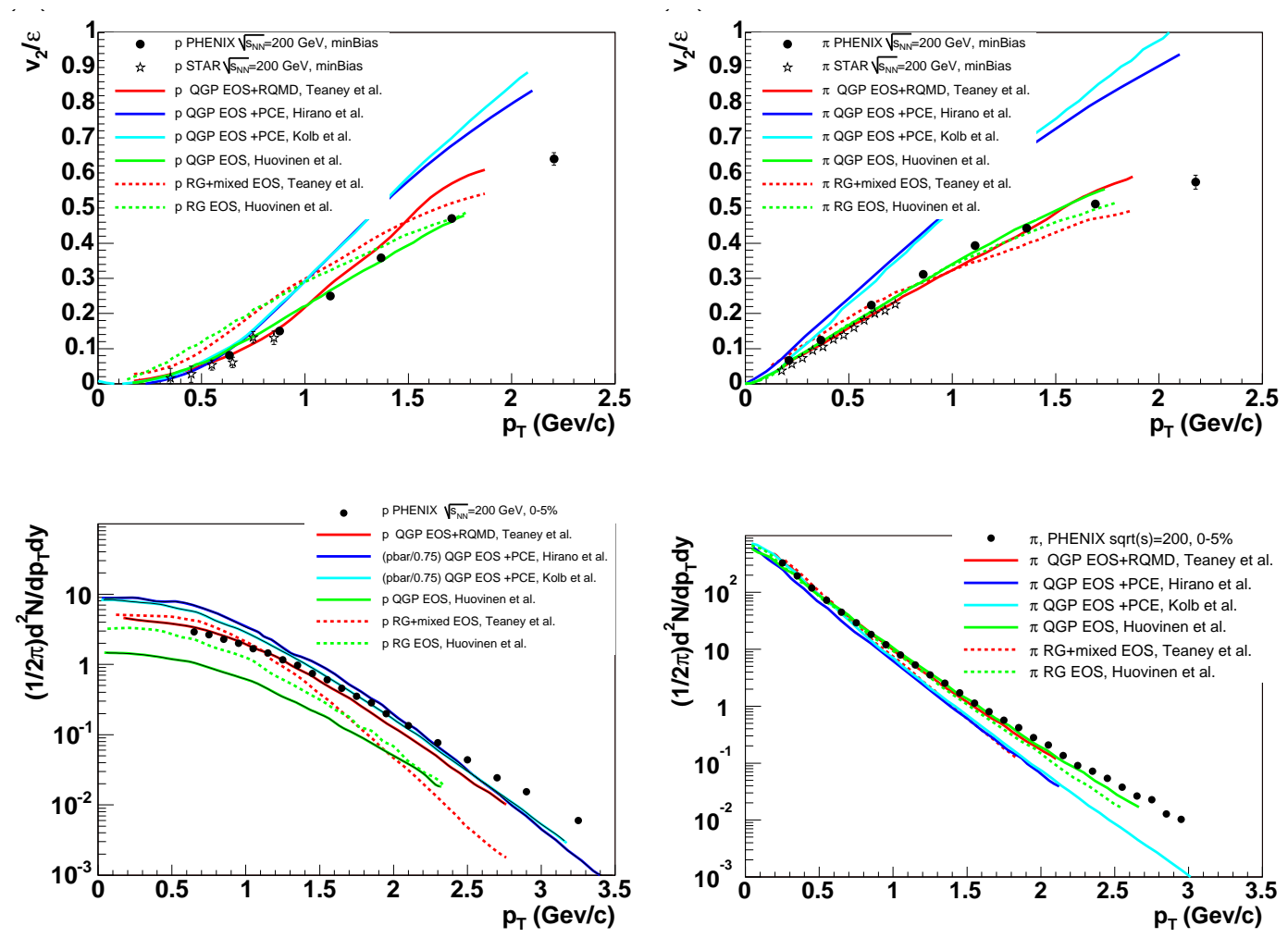

Fig. 20. Top two panels: On the left, proton $\frac{1}{\varepsilon} v_{2}\left(p_{T}\right)$ vs. $p_{T}$ for minimum-bias collisions at RHIC [50,103] are compared with hydro calculations $[106,118,120,107]$, and on the right is the same comparison for pions. Bottom two panels: On the left, proton spectra for 0-5\% collisions at RHIC [54] are compared with the same hydro calculations and on the the right is the same comparison for pions.

The same hydro models are compared to the pion $v_{2}\left(p_{T}\right) / \varepsilon$ measurements from STAR and PHENIX in Fig. 20. The Kolb (solid light-blue line) and Hirano (solid dark-blue line) calculations fail completely by predicting too strong a $v_{2}$. These two models have very similar partial chemical equilibrium assumptions in the late hadronic stage. It is worth noting that the Kolb calculation is the same as the Huovinen (solid green line) calculation with the exception of the final hadronic stage.

All the above models have assumed ideal hydrodynamics, i.e. with no viscosity and zero mean free path. Non-zero viscosity in the QGP reduces $v_{2}[121,122]$ and since the early hydro calculations from Teaney and Huovinen reproduced the magnitude of the pion $v_{2}$ data, it is often stated that viscosity of the matter at RHIC must be small [24]. However recent calculations from Hirano (3D) (solid dark-blue line) and Kolb (solid light-blue line) overpredict the measured $v_{2}$. As these results do not include dissipative effects, such as those resulting from hadronic interactions in the final state, their failure indicates that further work will be necessary before a quantitative determination of the 
viscosity in the QGP phase is possible. Progress will require both theoretical development and experimental measures that are less sensitive to how the azimuthal asymmetry of the energy-momentum tensor is distributed between different particles in the final stage of the reaction, e.g. the elliptic flow of the total transverse energy.

The same hydro models are now compared to the measured spectra from central collisions. The bottom right panel of Fig. 20 shows that all the hydro models reproduce the pion spectra below $p_{T} \sim 1 \mathrm{GeV} / c$; at higher $p_{T}$ the particles are less likely to be equilibrated and hydro models are not expected to work well. In the bottom left panel the calculated proton spectra from Huovinen [107] (solid green line) are lower than the data, due to the calculation maintaining chemical equilibrium throughout the hadronic phase. The lower temperature chemical freezeout suppresses the final calculated yield of heavier particles such as protons. Of the two calculations from Teaney [106] the calculation that includes the QGP phase (solid red line) reproduces the proton spectra, presumably because of the increased transverse flow from the stronger early pressure gradients. Hirano's and Kolb's (solid dark and lightblue lines) calculations break chemical equilibrium during the hadronic phase and overpredict the proton spectra at low $p_{T}$.

One difficulty is that the spectra comparison with hydrodynamic models is for central collisions while the $v_{2}$ comparison is for minimum-bias collisions. It is dificult to use central collisions for the $v_{2}$ comparison since the collisions are nearly symmetric and hence $v_{2}$ is small. In addition, hydrodyamic calculations that reproduce $v_{2}$ values over a broad range of centrality (from 0-45\% in Ref. [103]) tend to overpredict the data for more peripheral collisions by approximately $25 \%$, presumably because of a breakdown in the hydrodynamic assumptions. Hence when comparing to minimum-bias data sets, an overprediction of $v_{2}$ from the hydro models of less than $20 \%$ should be acceptable.

These comparisons between data and hydro models are summarized in Table 1 and in the following conclusions;

- $v_{2}\left(p_{T}, P I D\right)$ is sensitive to all stages of the reaction. Elliptic flow is produced by strong scattering in the initial phase, while the detailed shape of $v_{2}\left(p_{T}\right)$ and how the momentum asymmetry is distributed to different particles is affected by the transition from a QGP to hadronic phase and scattering in the final hadronic stage.

- The hydro models that reproduce the low- $p_{T}$ proton $v_{2}$ are those that include both a QGP and hadronic phase.

- The hadronic phase critically affects the final values of $v_{2}\left(p_{T}, P I D\right)$. Models (Hirano, Kolb) that include partial chemical equilibrium to reproduce the baryon yield, completely fail on the pion $v_{2}$.

- The only model that survives this comparison with measured $v_{2}$ and spectra 


\begin{tabular}{|c|c|c|c|c|c|c|}
\hline & \multicolumn{4}{|c|}{$Q G P+$ mixed $+R G$} & \multirow{2}{*}{$\frac{\text { mixed }+R G}{\text { Teaney }}$} & \multirow{2}{*}{$\frac{R G}{\text { Huovinen }}$} \\
\hline & Teaney & Hirano & Kolb & Huovinen & & \\
\hline Reference & {$[106]$} & {$[118]$} & {$[117,123]$} & {$[107]$} & {$[106]$} & {$[107]$} \\
\hline $\begin{array}{l}\text { latent } \\
\text { heat } \\
\left(\mathrm{GeV} / \mathrm{fm}^{3}\right)\end{array}$ & 0.8 & 1.7 & 1.15 & 1.15 & 0.8 & \\
\hline $\begin{array}{l}\text { init. } \varepsilon_{\max } \\
\left(\mathrm{GeV} / \mathrm{fm}^{3}\right)\end{array}$ & 16.7 & & 23 & 23 & 16.7 & 23 \\
\hline $\begin{array}{l}\text { init. } \quad\langle\varepsilon\rangle \\
\left(\mathrm{GeV} / \mathrm{fm}^{3}\right)\end{array}$ & 11.0 & 13.5 & & & 11.0 & \\
\hline$\tau_{0} \mathrm{fm} / c$ & 1.0 & 0.6 & 0.6 & 0.6 & 1.0 & 0.6 \\
\hline $\begin{array}{l}\text { hadronic } \\
\text { stage }\end{array}$ & RQMD & $\begin{array}{l}\text { partial } \\
\text { chemical } \\
\text { equil. }\end{array}$ & $\begin{array}{l}\text { partial } \\
\text { chemical } \\
\text { equil. }\end{array}$ & full equil. & RQMD & full equil. \\
\hline proton $\mathrm{v} 2$ & yes & $\begin{array}{ll}< & 0.7 \\
\mathrm{GeV} / c & \end{array}$ & $\begin{array}{ll}< & 0.7 \\
\mathrm{GeV} / c & \end{array}$ & yes & no & no \\
\hline pion v2 & yes & no & no & yes & yes & yes \\
\hline $\begin{array}{l}\text { proton } \\
\text { spectra }\end{array}$ & yes & overpredict & overpredict & no & no & no \\
\hline $\begin{array}{l}\text { pion spec- } \\
\text { tra }\end{array}$ & yes & $<1 \mathrm{GeV} / c$ & $<1 \mathrm{GeV} / c$ & yes & $\begin{array}{ll}< & 0.7 \\
\mathrm{GeV} / c & \end{array}$ & yes \\
\hline HBT & $\begin{array}{l}\text { Not avail- } \\
\text { able }\end{array}$ & No & No & No & $\begin{array}{l}\text { Not avail- } \\
\text { able }\end{array}$ & $\begin{array}{l}\text { Not avail- } \\
\text { able }\end{array}$ \\
\hline
\end{tabular}

Table 1

Summary of various hydro model assumptions and a comparison between measurements and hydro calculations. Two initial energies are tabulated, either the maximum energy density at the center of the collision or the energy density averaged over the transverse profile.

is Teaney's (solid red line) which includes a strong expansion in a QGP phase, a phase transition to a mixed phase, and then a hadronic cascade in the final hadronic state. There are open questions in this hybrid model, e.g. the sensitivity of the results to the matching conditions between hydro and RQMD. All other models fail in at least one $v_{2}$ or spectra comparison, partially due to differences in modeling the final hadronic state.

- Until the model uncertainty in the final state is reduced, it is not yet possible to use the measured splitting between proton and pion $v_{2}\left(p_{T}\right)$ to extract quantitative information on the EOS during the reaction, including the possible softening of the EOS due to the presence of a mixed phase. 
A comparison with the HBT data and some of the hydro models is shown in Fig. 21. It is unfortunate that not all hydro models have been compared to HBT data, e.g. the hydro+RQMD model from Teaney [106] has not been confronted with this observable. The hydro calculation from Kolb, Heinz and Huovinen [123] (solid green line) includes a first-order phase transition which leads to a long lifetime for the system. The source parameter $R_{\text {long }}$ is considered most sensitive to the duration of the whole collision, i.e. from initial overlap to final particle emission, and the Kolb/Huovinen hydro calculation [123] (solid green line) overpredicts the measured $R_{\text {long }}$ data. Changing to partial chemical equilibrium in the hadronic stage [118], indicated with the dark blue line, reduces the lifetime of the collision which improves the agreement with $R_{\text {long }}$. However the ratio $R_{\text {out }} / R_{\text {side }}$, which is sensitive to the duration over which particles are emitted, is still overpredicted.

There have been many attempts to understand what may be causing the disagreement with data (known collectively as the HBT puzzle);

- Sinyukov et al. [124] and Grassi et al. [125] have suggested that the sharp Cooper-Frye freezeout condition [126] should be replaced by an emission function that decouples hadrons depending on their hadronic cross section.

- However when this has been effectively implemented by using a hadronic cascade (URQMD) for the final hadronic stage, the predicted ratio $R_{\text {out }} / R_{\text {side }}$ increases and diverges further from the data [127]. Modeling the final stage with a hadronic cascade effectively includes dissipative effects which should increase the duration of emission and produce a larger ratio $R_{\text {out }} / R_{\text {side }}$.

- One method to reduce the lifetime of the reaction is to change the QGP EOS. Using a crossover instead of a first-order transition reduces the ratio $R_{\text {out }} / R_{\text {side }}$ by about $50 \%$ to $R_{\text {out }} / R_{\text {side }} \sim 1.5$ [128] which is still larger than the data. Because the calculation was restricted to $\eta=0$ Zschiesche et al. were unable to compare with the measured values of $R_{\text {long }}$.

In summary, model comparisons seem to be closer to the HBT data when the lifetime of the collision is made smaller than the long time resulting from a first-order phase transition. The small values of $R_{\text {out }} / R_{\text {side }}$ may indicate that there is little to no mixed phase present in the reactions. One possible direction for future comparisons with data is to include a more realistic EOS into the hydro models, e.g. to take the EOS from lattice QCD calculations [129]. Such a calculation needs to be compared with all the available data, including spectra and $v_{2}$, as well as HBT.

\subsection{Conclusions}

In summary we can make the following conclusions 

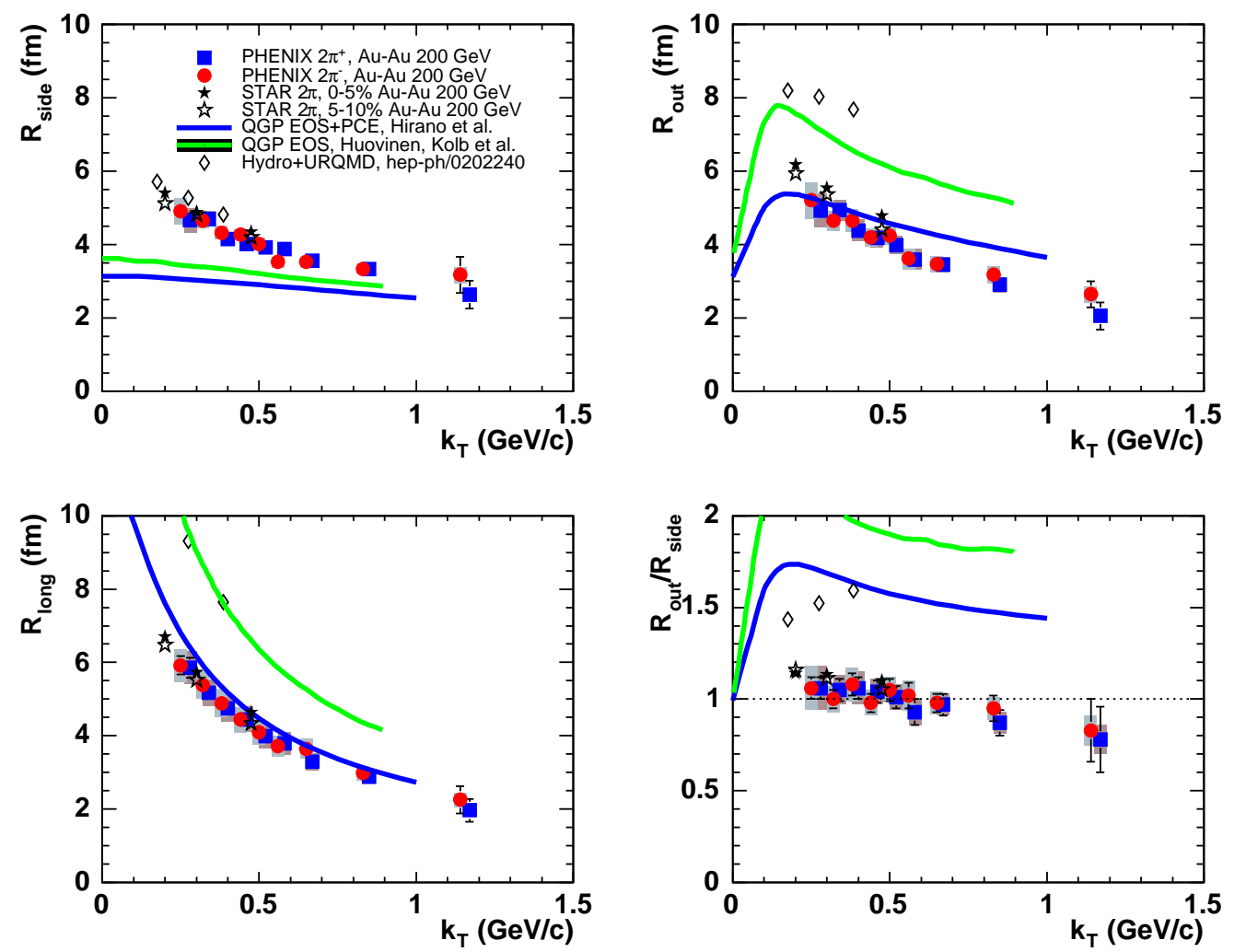

Fig. 21. The $k_{T}$ dependence of the Bertsch-Pratt parameters for $\pi^{+} \pi^{+}$(blue square) and $\pi^{-} \pi^{-}$(red circle) for 0-30\% centrality with statistical error bars and systematic error bands. Results from PHENIX [56], STAR [114] and hydrodynamics models (Hirano [118], Kolb/Huovinen [123] and Soff [130], diamonds) are overlaid.

- The measured yields and spectra of hadrons are consistent with thermal emission from a strongly expanding source.

- Strangeness is fully saturated at RHIC, consistent with full chemical equilibrium.

- The scaling of $v_{2}$ with eccentricity shows that collective behavior is established early in the collision.

- Elliptic flow is stronger at RHIC than at the SPS, since the measured slope of $v_{2}\left(p_{T}\right)$ for pions is $50 \%$ larger at RHIC.

- The measured proton $v_{2}\left(p_{T}\right)$ is less than that for pions at low $p_{T}$; the small magnitude of the proton $v_{2}$ at low $p_{T}$ is reproduced by hydro models that include both a QGP and hadronic phase.

- However several of the hydro models that reproduce the proton $v_{2}\left(p_{T}\right)$ fail for the pion $v_{2}\left(p_{T}\right)$.

- The HBT source parameters, especially the small value of $R_{\text {long }}$ and the ratio $R_{\text {out }} / R_{\text {side }}$, suggest that the mixed phase is too long-lived in the current hydro calculations. 
Hence we currently do not have a consistent picture of the space-time dynamics of reactions at RHIC as revealed by spectra, $v_{2}$, and HBT. The lack of a consistent picture of the dynamics means that it is not yet possible to extract quantitative properties of the QGP or mixed phase using the observables $v_{2}$, spectra, or HBT.

\section{FLUCTUATIONS}

\subsection{Net-Charge Fluctuations}

In the study of the fluctuations of multiplicity as a means to understand the dynamics of charged particle production, one important realization was to use small regions of phase space, where energy-momentum conservation constraints would not be significant $[131,132,133]$. Such studies led to the important observation that the distribution of multiplicity, even in small intervals near mid-rapidity, was Negative Binomial rather than Poisson, which indicated large multiplicity correlations even in small $\delta \eta$ intervals [134]. No such studies are yet available at RHIC.

Based on predictions that event-by-event fluctuations of the net charge in local phase space regions would show a large decrease as a signature of the QGP [135,136,137], net-charge fluctuations were measured in PHENIX [44]. The idea is that in a QGP composed of fractionally charged quarks, the larger number of fractionally charged particles compared to unit-charged hadrons would result in smaller relative net-charge fluctuations in a QGP than for a pure gas of hadrons and that this original fluctuation would survive the transition back to ordinary hadrons.

It is important to realize that the study of net-charge fluctuations represents the study of fluctuations in a quantity that is conserved over all phase space. Consider $N=N_{+}+N_{-}$charged particles produced in the full phase space. By charge conservation $N_{+}=N_{-}=N / 2$, and the net charge $Q \equiv N_{+}-N_{-}$ is identically zero so that there are no net-charge fluctuations - the variance $V(Q)=0$, where

$$
V(Q) \equiv\left\langle Q^{2}\right\rangle-\langle Q\rangle^{2}
$$

In a smaller region of phase space, where $p$ is the fraction of $N$ observed in a stochastic scenario, the mean and variance of the number of positive $n_{+}$and negative $n_{-}$particles are equal, but the variance of $Q$ is no longer identically 
zero:

$$
\begin{aligned}
& \left\langle n_{+}\right\rangle=\left\langle n_{-}\right\rangle=p N / 2, \\
& V\left(n_{+}\right)=V\left(n_{-}\right)=p(1-p) N / 2,
\end{aligned}
$$

from which it follows that

$$
V(Q)=(1-p) n_{c h}
$$

where $n_{c h}=p N$ is the expected number of charged particles on the interval. Thus the normalized variance in $Q$ (normalized to Poisson statistics) is defined as:

$$
v(Q)=\frac{V(Q)}{n_{c h}}=(1-p)
$$

In the limit $n_{c h} \gg 0$, the variance of the charge ratio $R=n_{+} / n_{-}$approaches $V(R)=4(1-p) / n_{c h}$. However, it is well known in mathematical statistics that moments of the inverse of a stochastic variable, e.g. $1 / n_{-}$, diverge if there is any finite probability, no matter how small, for $n_{-}=0$. Thus, the charge ratio is not a stable measure of fluctuations.

The previous arguments are based on fixed $N$. The results where $N$ varies according to a specified distribution are also interesting. If $n_{-}$is Poisson distributed, with mean value $\mu=N / 2$ over the whole phase space, then in the region of phase space with probability $p$ the distribution is also Poisson, with mean $\left.\left\langle n_{-}\right\rangle\right|_{p}=\mu_{p}=p N / 2$. If, on the other hand $n_{-}$is Negative Binomial distributed, with mean value $\mu=N / 2$ and NBD parameter $\sigma^{2} / \mu^{2}-1 / \mu=1 / k$ for the whole phase space, then in the region of phase space with probability $p$, the distribution is Negative Binomial with mean $\left.\left\langle n_{-}\right\rangle\right|_{p}=\mu_{p}=p N / 2$ and the same value of $1 / k$.

Actually, the binomial division preserves $\sigma_{p}^{2} / \mu_{p}^{2}-1 / \mu_{p}=1 / k$, for any distribution [138]. This appears to indicate that smaller intervals, which tend to have larger values of $\sigma_{p}^{2} / \mu_{p}^{2}$ would be less sensitive to the global $1 / k$, the long-range correlation. This would be true except for the fact that there are short-range correlations which are better seen on small intervals of phase space. Another important thing to note regarding a binomial split of a Negative Binomial distribution is that the two subintervals are not statistically independent. The conditional probability distribution on the interval $(1-p)$ depends upon the outcome on the interval $p$ [139]. It is unfortunate that these elegant arguments can not be applied to the net-charge fluctuations since $\langle Q\rangle=0$. 
The PHENIX measurement [44] of the normalized variance $v(Q)$ of net-charge fluctuations is shown in Fig. 22 in the interval $-0.35 \leq \delta \eta \leq+0.35$ as a function of the azimuthal angular interval of reconstructed tracks, either at the detector, $\Delta \phi_{d}$, or at the vertex, $\Delta \phi_{r}$, chosen symmetrically around the detector acceptance. For smaller $\Delta \phi_{r}$ the data agree with the purely stochastic $(1-p)$ dependence shown as the solid line, but deviate from the stochastic prediction at larger values due to correlations from resonance decay, such as $\rho^{0} \rightarrow \pi^{+}+\pi^{-}$as nicely explained by RQMD [140].

Absent new theoretical insight, it is difficult to understand how quark-level net-charge fluctuations in a QGP can be related to net-charge fluctuations of hadrons, where, by definition, strong correlations exist, e.g., in the formation of a meson from a $q-\bar{q}$ pair. Also, the study of the fluctuations of net charge, which is conserved, may not be as useful to detect interesting fluctuations as the study of fluctuations of the total charged multiplicity, which is much less constrained by conservation laws. This has yet to be tried at RHIC.

\subsection{Event-by-Event Average-p $p_{T}$ Fluctuations}

Fluctuations in the event-by-event average $p_{T}$, denoted $M_{p_{T}}$, have been measured and provide a severely small limit on possible fluctuations from a sharp phase transition. For events with $n$ detected charged particles with magnitudes of transverse momenta, $p_{T_{i}}$, the event-by-event average $p_{T}$, denoted $M_{p_{T}}$ is defined as:

$$
M_{p_{T}}=\overline{p_{T}}=\frac{1}{n} \sum_{i=1}^{n} p_{T_{i}} .
$$

Mixed events are used to define the baseline for random fluctuations of $M_{p_{T}}$ in PHENIX $[45,55]$. This has the advantage of effectively removing any residual detector-dependent effects. The event-by-event average distributions are very sensitive to the number of tracks in the event (denoted $n$ or $N_{\text {tracks }}$ ), so the mixed event sample is produced with the identical $N_{\text {tracks }}$ distribution as the data. Additionally, no two tracks from the same data event are placed in the same mixed event in order to remove any intra-event correlations in $p_{T}$. Finally, $\left\langle M_{p_{T}}\right\rangle$ must exactly match the semi-inclusive $\left\langle p_{T}\right\rangle$.

For the case of statistical independent emission, where the fluctuations are purely random, an analytical formula for the distribution in $M_{p_{T}}$ can be obtained assuming Negative Binomial distributed event-by-event multiplicity, with Gamma distributed semi-inclusive $p_{T}$ spectra [141]. The formula depends on the four semi-inclusive parameters $\langle n\rangle, 1 / k, b$ and $p$ which are derived from the means and standard deviations of the semi-inclusive $p_{T}$ and multiplicity 

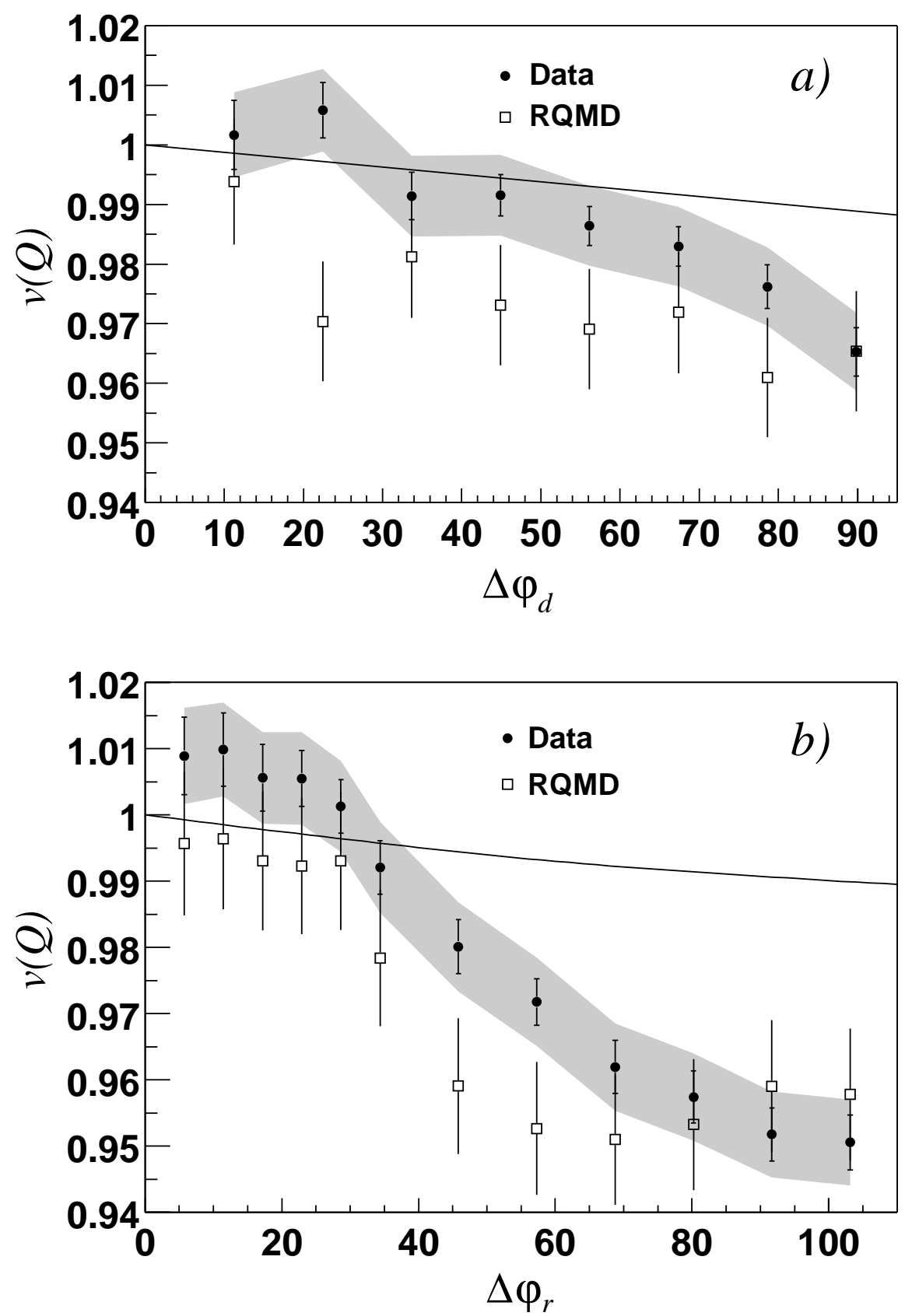

Fig. 22. $v(Q)$ for the $10 \%$ most central events in data and RQMD, as a function of the azimuthal interval in degrees of reconstructed tracks, either a) at the detector, $\Delta \phi_{d}$, or b) at the vertex, $\Delta \phi_{r}$, chosen symmetrically around the detector acceptance. For data, the error band shows the total statistical error, whereas the error bars indicate the uncorrelated part. The solid line shows the expected reduction in $v(Q)$ in the stochastic scenario when global charge conservation is taken into account [44]. 
distributions, $\langle n\rangle, \sigma_{n},\left\langle p_{T}\right\rangle, \sigma_{p_{T}}$ :

$$
f(y)=\sum_{n=n_{\min }}^{n_{\max }} f_{\mathrm{NBD}}(n, 1 / k,\langle n\rangle) f_{\Gamma}(y, n p, n b),
$$

where $y=M_{p_{T}}$. For fixed $n$, and purely random fluctuations, the mean and standard deviation of $M_{p_{T}}$ follow the expected behavior, $\left\langle M_{p_{T}}\right\rangle=\left\langle p_{T}\right\rangle$, $\sigma_{M_{p_{T}}}=\sigma_{p_{T}} / \sqrt{n}$. In PHENIX, Eq. 16 is used to confirm the randomness of mixed events which are used to define the baseline for random fluctuations of $M_{p_{T}}[45,55]$.

The measured $M_{p_{T}}$ distributions for the data in two centrality classes for $\sqrt{s_{N N}}=200 \mathrm{GeV} \mathrm{Au}+\mathrm{Au}$ collisions in PHENIX [55] are shown in Fig. 23 (data points) compared to the mixed-event distributions (histograms). The non-Gaussian, Gamma-distribution shape of the $M_{p_{T}}$ distributions is evident. The difference between the data and the mixed-event random baseline distributions is not visible to the naked eye. The nonrandom fluctuation is quantified by the fractional difference of $\omega_{p_{T}}$, the normalized standard deviation of $M_{p_{T}}$, for the data and the mixed-event (random) samples:

$$
\begin{aligned}
\omega_{p_{T}} & =\frac{\sigma_{M_{p_{T}}}}{\left\langle M_{p_{T}}\right\rangle}, \\
F_{p_{T}} & =\frac{\omega_{p_{T}, \text { data }}-\omega_{p_{T}, \text { mixed }}}{\omega_{p_{T}, \text { mixed }}} .
\end{aligned}
$$

The results are shown as a function of centrality, represented by $N_{\text {part }}$ in Fig. 24 .

The dependence of $F_{p_{T}}$ on $N_{\text {part }}$ is striking. To further understand this dependence and the source of these nonrandom fluctuations, $F_{p_{T}}$ was measured over a varying $p_{T}$ range, $0.2 \mathrm{GeV} / c \leq p_{T} \leq p_{T}^{\max }$ (Fig. 25), where $p_{T}^{\max }=2.0$ $\mathrm{GeV} / c$ for the $N_{\text {part }}$ dependence.

The increase of $F_{p_{T}}$ with $p_{T}^{\max }$ suggests elliptic flow or jet origin. This was investigated using a Monte Carlo simulation of correlations due to elliptic flow and jets in the PHENIX acceptance. The flow was significant only in the lowest centrality bin and negligible $\left(F_{p_{T}}<0.1 \%\right)$ at higher centralities. Jets were simulated by embedding (at a uniform rate per generated particle, $\left.S_{\text {prob }}\left(N_{\text {part }}\right)\right) p+p$ hard-scattering events from the PYTHIA event generator into simulated $\mathrm{Au}+\mathrm{Au}$ events assembled at random according to the measured $N_{\text {tracks }}$ and semi-inclusive $p_{T}$ distributions. This changed $\left\langle p_{T}\right\rangle$ and $\sigma_{p_{T}}$ by less than $0.1 \%$. $S_{\text {prob }}\left(N_{\text {part }}\right)$ was either constant for all centrality classes, or scaled by the measured hard-scattering suppression factor $R_{A A}\left(N_{\text {part }}\right)$ for $p_{T}>4.5$ $\mathrm{GeV} / c$ [49]. A value $F_{p_{T}}=2.06 \%$ for $p+p$ collisions was extracted from 

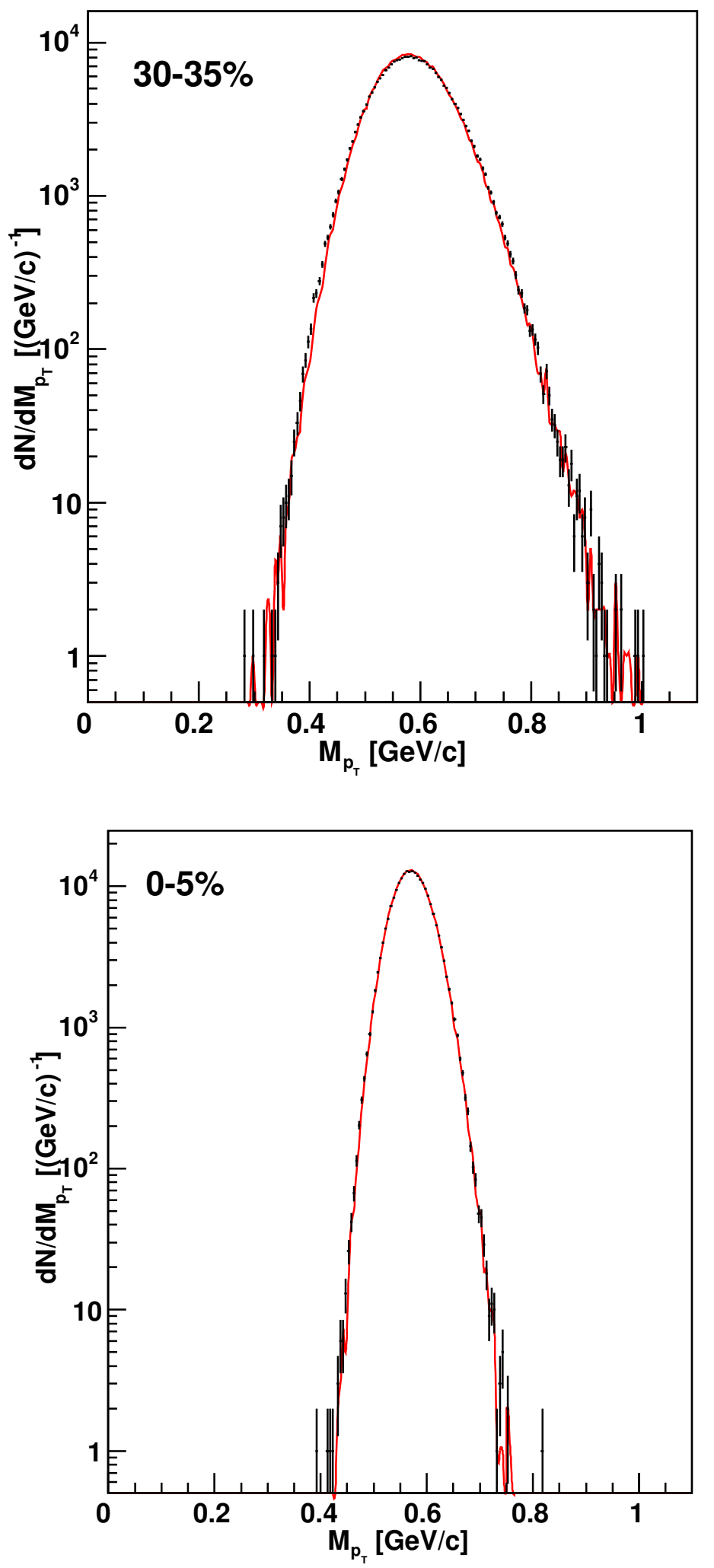

Fig. 23. $M_{p_{T}}$ for $30-35 \%$ and $0-5 \%$ centrality classes: data (points) mixed events (histogram) [55]. 


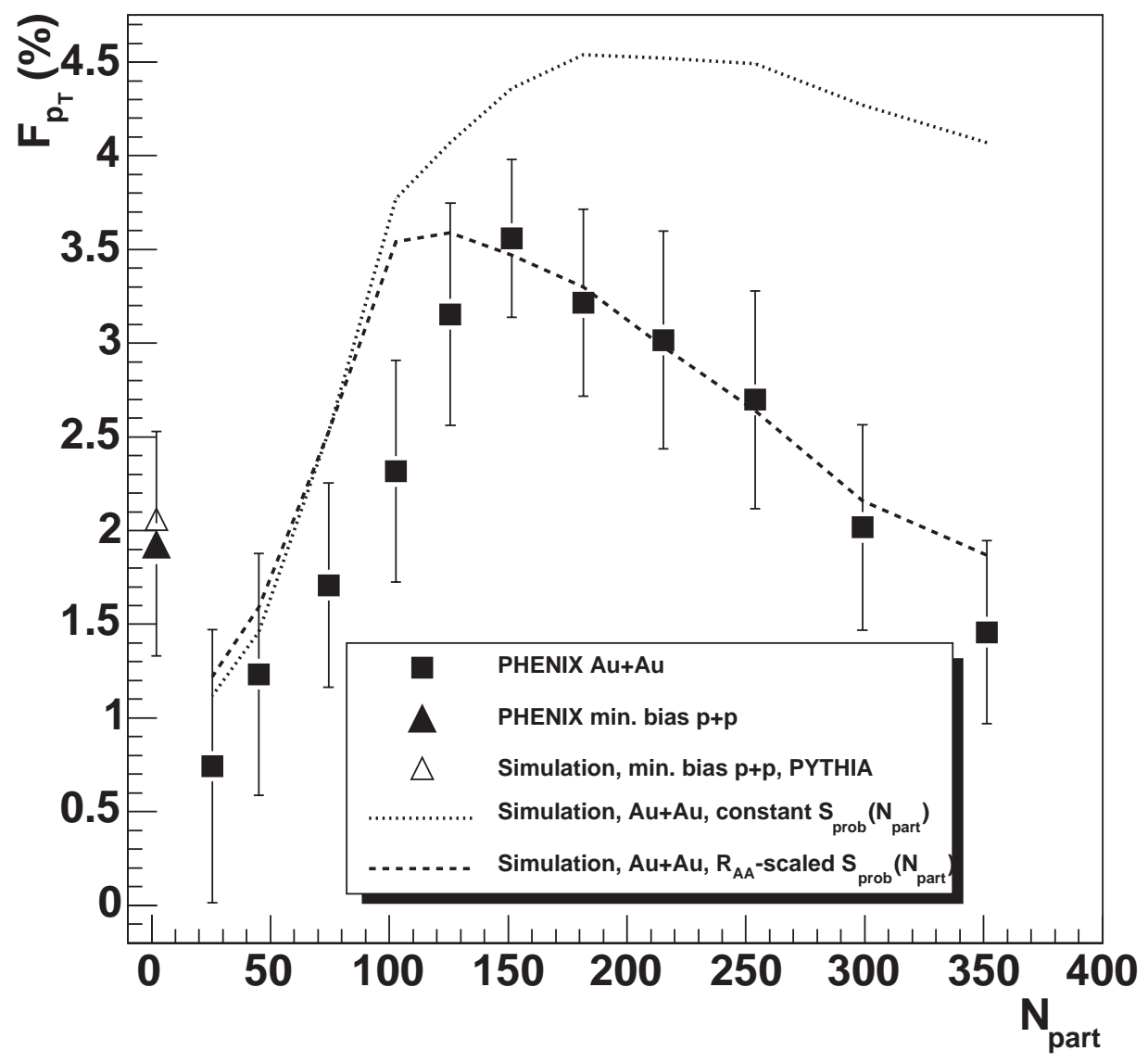

Fig. 24. $F_{p_{T}}$ vs. centrality, represented as the average number of participants $\left(N_{\text {part }}\right)$ in a centrality class, compared to jet simulation [55].

pure PYTHIA events in the PHENIX acceptance in agreement with the $p+$ $p$ measurement (Fig. 24). The value of $S_{\text {prob }}\left(N_{\text {part }}\right)$ was chosen so that the simulation with $S_{\text {prob }}\left(N_{\text {part }}\right) \times R_{A A}\left(N_{\text {part }}\right)$ agreed with the data at $N_{\text {part }}=$ 182. The centrality and $p_{T}^{\max }$ dependences of the measured $F_{p_{T}}$ match the simulation very well, but only when the $R_{A A}$ scaling is included.

A less experiment-dependent method to compare nonrandom fluctuations is to assume that the entire $F_{p_{T}}$ is due to temperature fluctuations of the initial state, with RMS variation $\sigma_{T} /\langle T\rangle[142,45]$. Then,

$$
\omega_{p_{T}, \text { data }}^{2}-\omega_{p_{T} \text {,mixed }}^{2}=\left(1-\frac{1}{\langle n\rangle}\right) \frac{\sigma_{T}^{2}}{\langle T\rangle^{2}}=2 F_{p_{T}} \omega_{p_{T}, \text { mixed }}^{2},
$$

This yields $\sigma_{T} /\langle T\rangle=1.8 \%$ for central collisions in PHENIX with similarly small values for the other Relativistic Heavy Ion experiments [143], 1.7\% in 


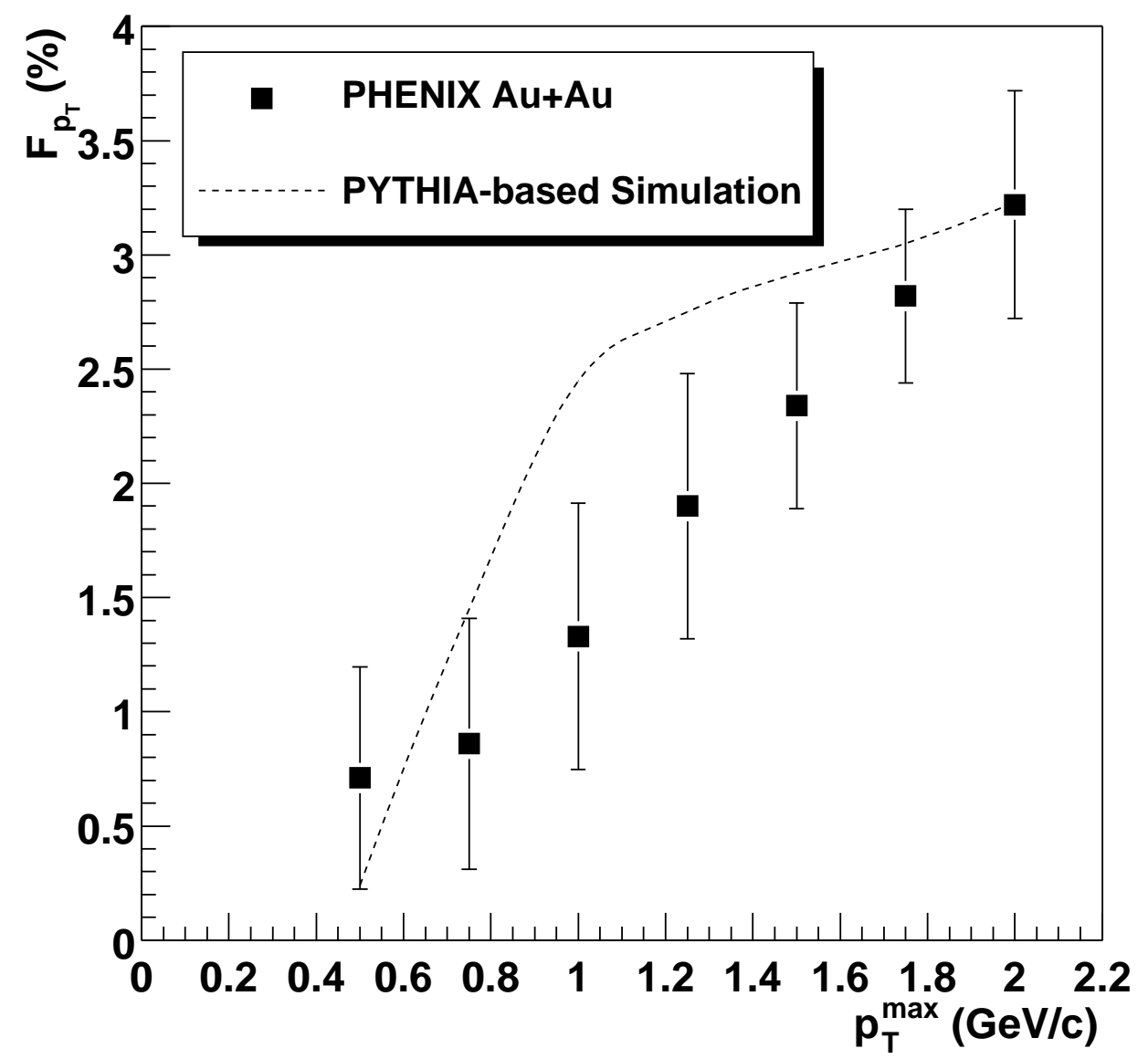

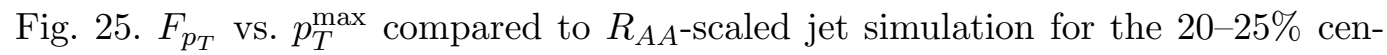
trality class $\left(N_{\text {part }}=181.6\right)[55]$.

STAR, $1.3 \%$ in CERES, and $0.6 \%$ in NA49. These results put severely small limits on the critical fluctuations that were expected for a sharp phase transition, both at SPS energies and at RHIC, but are consistent with the expectation from lattice QCD that the transition is a smooth crossover [12].

Other proposed explanations of the centrality and $p_{T}^{\max }$ dependences of $F_{p_{T}}$ include: overlapping color strings which form clusters so that the number of sources and $\left\langle p_{T}\right\rangle$ per source is modified as a function of centrality [144]; and near equilibrium $p_{T}$ correlations induced by spatial inhomogeniety [145].

\subsection{Conclusions}

Critical behavior near the phase boundary can produce nonrandom fluctuations in observables such as the net-charge distribution and the average trans- 
verse momentum distribution. Our search for net-charge fluctuations has ruled out the most naive model of charge fluctuations in a QGP, but it is unclear whether the charge fluctuation signature can survive hadronization. Our measurement of the event-by-event average $p_{T}$ distribution shows a nonrandom fluctuation that is consistent with the effect expected from high- $p_{T}$ jets. This puts a severe constraint on the critical fluctuations that were expected for a sharp phase transition but is consistent with the expectation from lattice QCD that the transition is a smooth crossover [12].

\section{BINARY SCALING}

\subsection{Hard Scattering and $p Q C D$}

One way to get a partonic probe into the midst of an $A+A$ collision is to use the high- $p_{T}$ partons produced by hard scattering. For $p+p$ collisions in the RHIC energy range, hard scattering is considered to be the dominant process of particle production with $p_{T} \geq 2 \mathrm{GeV} / c$ at mid-rapidity. Typically, particles with $p_{T} \geq 2 \mathrm{GeV} / c$ are produced from states with two roughly back-to-back jets which are the result of scattering of constituents of the nucleons (partons) as described by pQCD [146].

The overall $p+p$ hard-scattering cross section in "leading logarithm" pQCD is the sum over parton reactions $a+b \rightarrow c+d$ (e.g. $g+q \rightarrow g+q$ ) at parton-parton center-of-mass (c.m.) energy $\sqrt{\hat{s}}$ :

$$
\frac{d^{3} \sigma}{d x_{1} d x_{2} d \cos \theta^{*}}=\frac{1}{s} \sum_{a b} f_{a}\left(x_{1}\right) f_{b}\left(x_{2}\right) \frac{\pi \alpha_{s}^{2}\left(Q^{2}\right)}{2 x_{1} x_{2}} \Sigma^{a b}\left(\cos \theta^{*}\right),
$$

where $f_{a}\left(x_{1}\right), f_{b}\left(x_{2}\right)$, are parton distribution functions, the differential probabilities for partons $a$ and $b$ to carry momentum fractions $x_{1}$ and $x_{2}$ of their respective protons (e.g. $u\left(x_{2}\right)$ ), and where $\theta^{*}$ is the scattering angle in the partonparton c.m. system. The parton-parton c.m. energy squared is $\hat{s}=x_{1} x_{2} s$, where $\sqrt{s}$ is the c.m. energy of the $p+p$ collision. The parton-parton c.m. system moves with rapidity $y=1 / 2 \ln \left(x_{1} / x_{2}\right)$ in the $p+p$ c.m. system.

Equation 20 gives the $p_{T}$ spectrum of outgoing parton $c$, which then fragments into hadrons, e.g. $\pi^{0}$. The fragmentation function $D_{c}^{\pi^{0}}\left(z, \mu^{2}\right)$ is the probability for a $\pi^{0}$ to carry a fraction $z=p^{\pi^{0}} / p^{c}$ of the momentum of outgoing parton $c$. Equation 20 must be summed over all subprocesses leading to a $\pi^{0}$ in the final state. The parameter $\mu^{2}$ is an unphysical "factorization" scale introduced to account for collinear singularities in the structure and fragmentation functions $[146,147]$. 
In this formulation, $f_{a}\left(x_{1}, \mu^{2}\right), f_{b}\left(x_{2}, \mu^{2}\right)$ and $D_{c}^{C}\left(z, \mu^{2}\right)$ represent the "longdistance phenomena" to be determined by experiment; while the characteristic subprocess angular distributions, $\Sigma^{a b}\left(\cos \theta^{*}\right)$, and the coupling constant, $\alpha_{s}\left(Q^{2}\right)=\frac{12 \pi}{25} \ln \left(Q^{2} / \Lambda^{2}\right)$, are fundamental predictions of QCD [148,149,150] for the short-distance, large- $Q^{2}$, phenomena. The momentum scale $Q^{2} \sim p_{T}^{2}$ for the scattering subprocess, while $Q^{2} \sim \hat{s}$ for a Compton or annihilation subprocess, but the exact meaning of $Q^{2}$ tends to be treated as a parameter rather than a dynamical quantity. The transverse momentum of a scattered constituent is:

$$
p_{T}=p_{T}^{*}=\frac{\sqrt{\hat{s}}}{2} \sin \theta^{*} .
$$

Equation 20 leads to a general ' $x_{T}$-scaling' form for the invariant cross section of high- $p_{T}$ particle production:

$$
E \frac{d^{3} \sigma}{d^{3} p}=\frac{1}{p_{T}^{n}} F\left(x_{T}\right)=\frac{1}{\sqrt{s}^{n}} G\left(x_{T}\right),
$$

where $x_{T}=2 p_{T} / \sqrt{s}$. The cross section has two factors, a function $F\left(x_{T}\right)$ $\left(G\left(x_{T}\right)\right)$ which 'scales', i.e. depends only on the ratio of momenta, and a dimensioned factor, $1 / p_{T}^{n}\left(1 / \sqrt{s}^{n}\right)$, where $n$ equals 4 in lowest-order (LO) calculations, analogous to the $1 / q^{4}$ form of Rutherford Scattering in QED. The structure and fragmentation functions are all in the $F\left(x_{T}\right)\left(G\left(x_{T}\right)\right)$ term. Due to higher-order effects such as the running of the coupling constant, $\alpha_{s}\left(Q^{2}\right)$, the evolution of the structure and fragmentation functions, and the initialstate transverse momentum $k_{T}, n$ is not a constant but is a function of $x_{T}$, $\sqrt{s}$. Measured values of $n\left(x_{T}, \sqrt{s}\right)$ in $p+p$ collisions are between 5 and 8 .

\subsection{Mid-Rapidity $p_{T}$ Spectra from $p+p$ Collisions}

The scaling and power-law behavior of hard scattering are evident from the $\sqrt{s}$ dependence of the $p_{T}$ dependence of the $p+p$ invariant cross sections. This is shown for nonidentified charged hadrons, $\left(h^{+}+h^{-}\right) / 2$, in Fig. 26a. At low $p_{T} \leq 1 \mathrm{GeV} / c$ the cross sections exhibit a "thermal" $\exp \left(-6 p_{T}\right)$ dependence, which is largely independent of $\sqrt{s}$, while at high $p_{T}$ there is a power-law tail, due to hard scattering, which depends strongly on $\sqrt{s}$. The characteristic variation with $\sqrt{s}$ at high $p_{T}$ is produced by the fundamental power-law and scaling dependence of Eqs. 20, 22. This is best illustrated by a plot of

$$
\sqrt{s}^{n\left(x_{T}, \sqrt{s}\right)} \times E \frac{d^{3} \sigma}{d^{3} p}=G\left(x_{T}\right),
$$



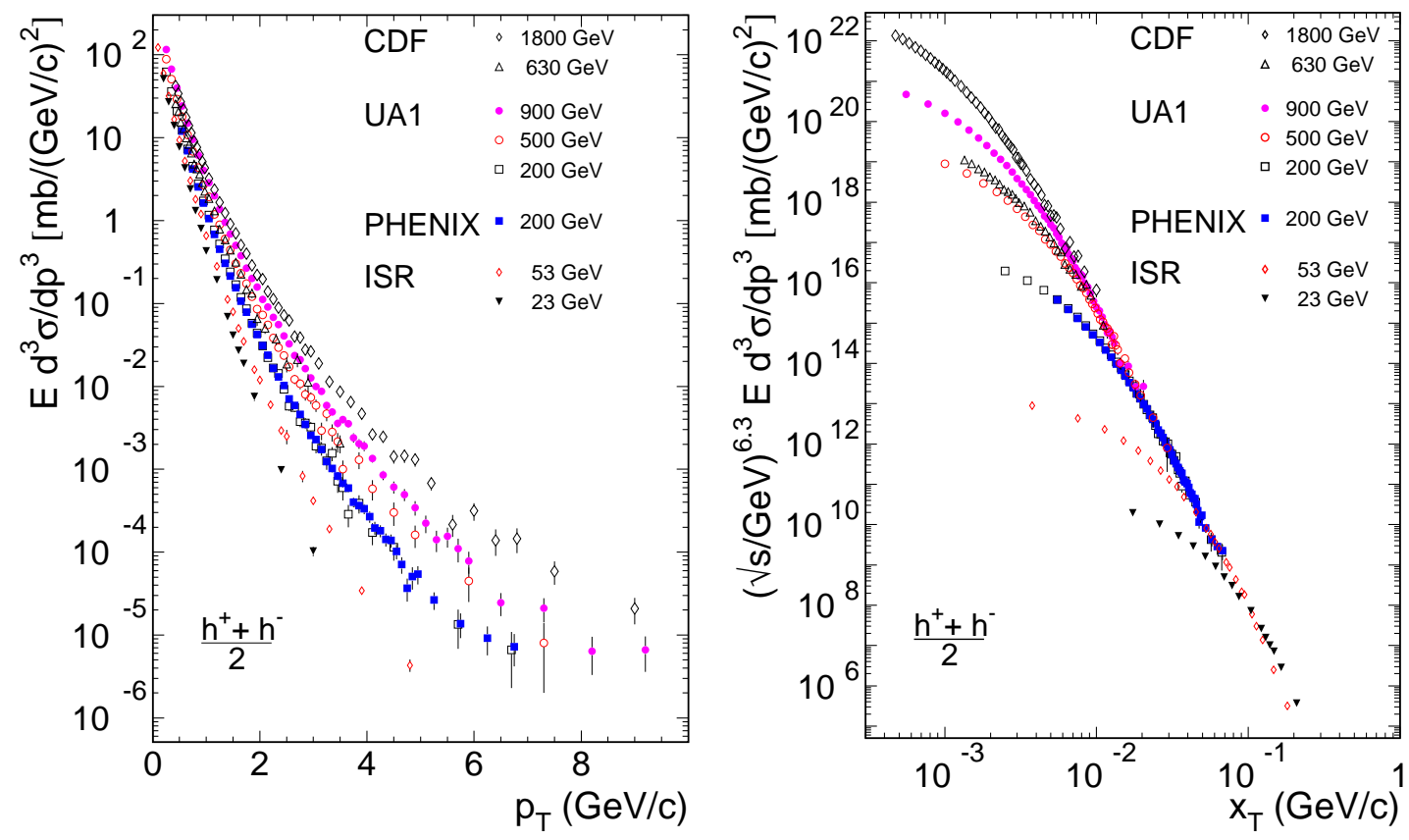

Fig. 26. (left) $E d^{3} \sigma\left(p_{T}\right) / d^{3} p$ at mid-rapidity as a function of $\sqrt{s}$ in $p+p$ collisions. (right) $\sqrt{s}(\mathrm{GeV})^{6.3} \times E d^{3} \sigma / d^{3} p$ vs. $x_{T}=2 p_{T} / \sqrt{s}$ [53] (and references therein).

as a function of $x_{T}$, with $n\left(x_{T}, \sqrt{s}\right)=6.3$, which is valid for the $x_{T}$ range of the present RHIC measurements (Fig. 26b). The data show an asymptotic power law with increasing $x_{T}$. Data at a given $\sqrt{s}$ fall below the asymptote at successively lower values of $x_{T}$ with increasing $\sqrt{s}$, corresponding to the transition region from hard to soft physics in the $p_{T}$ region of about $2 \mathrm{GeV} / c$.

The PHENIX measurement of the invariant cross section for $\pi^{0}$ production in $p+p$ collisions at $\sqrt{s}=200 \mathrm{GeV}$ [60] agrees with NLO pQCD predictions over the range $2.0 \leq p_{T} \leq 15 \mathrm{GeV} / c$ (Fig. 27).

\subsection{Scaling Hard Scattering from $p+p$ to $p+A$ and $A+B$ Collisions}

Since hard scattering is point like, with distance scale $1 / p_{T} \leq 0.1 \mathrm{fm}$, and the hard-scattering cross section factorizes as shown in Eq. 20, the cross section in $p+A$ or $A+B$ collisions, compared to $p+p$, is proportional to the relative number of possible point-like encounters. The number of encounters of pointlike constituents of nucleons is then proportional to $A(A B)$, for $p+A(A+B)$ minimum-bias collisions. For $A+B$ collisions at impact parameter $b$, it is proportional to $T_{A B}(b)$, the nuclear thickness function, which is the integral of the product of nuclear thickness over the geometrical overlap region of the two nuclei. In detail, the semi-inclusive invariant yield of e.g. high- $p_{T} \pi^{0}$ 's for $A+B$ inelastic collisions, with centrality $f$, is related to the $p+p$ cross section 

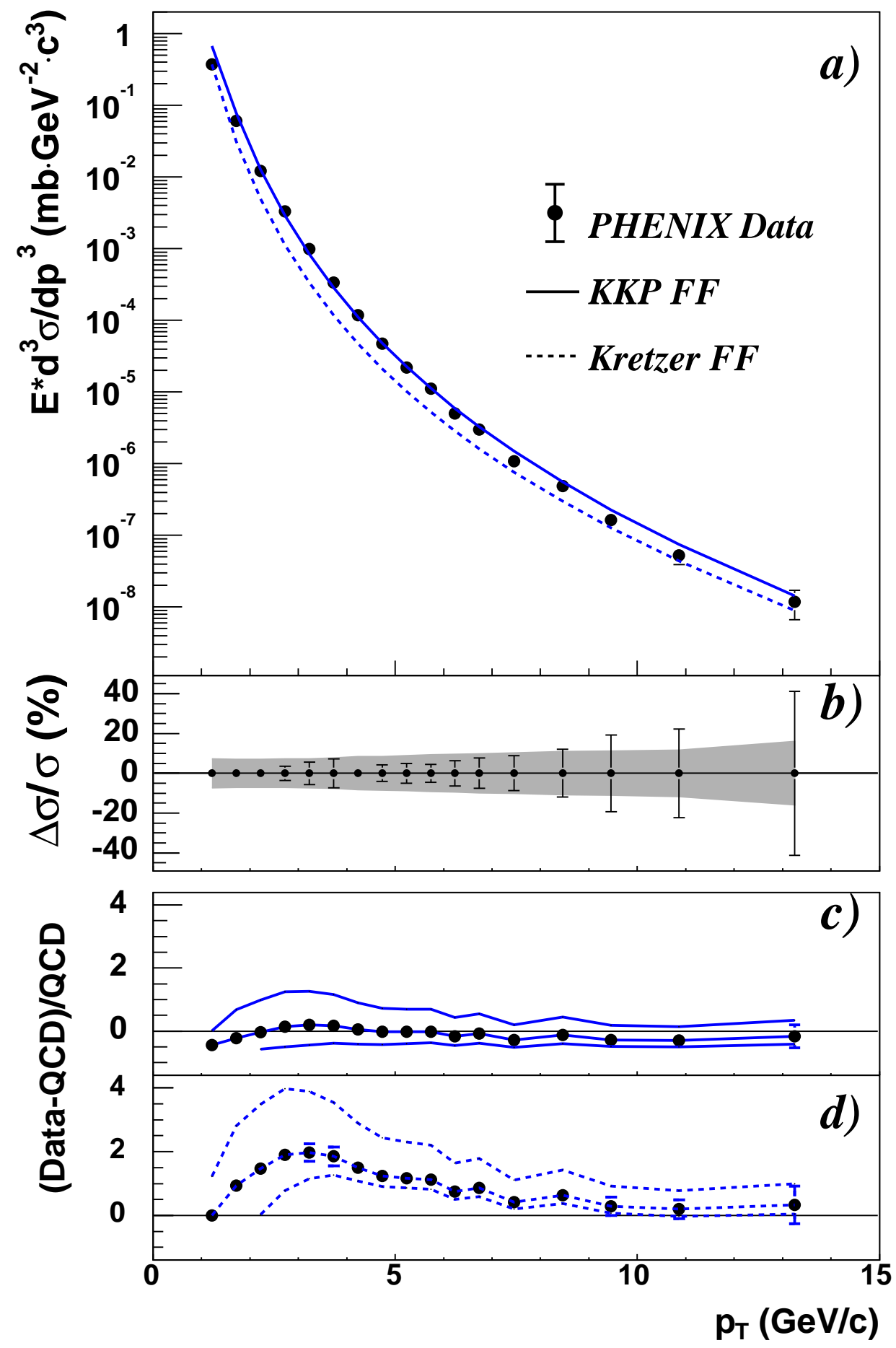

Fig. 27. PHENIX $\pi^{0}$ invariant cross section at mid-rapidity from $p+p$ collisions at $\sqrt{s}=200 \mathrm{GeV}$, together with NLO pQCD predictions from Vogelsang [151,152]. a) The invariant differential cross section for inclusive $\pi^{\circ}$ production (points) and the results from NLO pQCD calculations with equal renormalization and factorization scales of $p_{T}$ using the "Kniehl-Kramer-Pötter" (solid line) and "Kretzer" (dashed line) sets of fragmentation functions. b) The relative statistical (points) and point-to-point systematic (band) errors. c,d) The relative difference between the data and the theory using KKP (c) and Kretzer (d) fragmentation functions with scales of $p_{T} / 2$ (lower curve), $p_{T}$, and $2 p_{T}$ (upper curve). In all figures, the normalization error of $9.6 \%$ is not show 960$]$. 
by:

$$
\left.\frac{1}{N_{A B}^{\mathrm{evt}}} \frac{d^{2} N_{A B}^{\pi^{0}}}{d p_{T} d y}\right|_{f}=\left\langle T_{A B}\right\rangle_{f} \times \frac{d^{2} \sigma_{p p}^{\pi^{0}}}{d p_{T} d y} .
$$

Note that

$$
\left\langle T_{A B}\right\rangle_{f}=\frac{\int_{f} T_{A B}(b) d^{2} b}{\int_{f}\left(1-e^{-\sigma_{N N} T_{A B}(b)}\right) d^{2} b}=\frac{\left\langle N_{c o l l}\right\rangle_{f}}{\sigma_{N N}},
$$

where $\left\langle N_{\text {coll }}\right\rangle_{f}$ is the average number of binary nucleon-nucleon inelastic collisions, with cross section $\sigma_{N N}$, in the centrality class $f$. This leads to the description of the scaling for point-like processes as binary-collision (or $N_{\text {coll }}$ ) scaling.

Nuclear medium effects, either in the initial or final state, can modify the expected scaling. These modifications can be quantitatively studied by measurement of the Nuclear modification factor $R_{A B}$, which is defined as

$$
R_{A B}=\frac{d N_{A B}^{P}}{\left\langle T_{A B}\right\rangle_{f} \times d \sigma_{N N}^{P}}=\frac{d N_{A B}^{P}}{\left\langle N_{c o l l}\right\rangle_{f} \times d N_{N N}^{P}},
$$

where $d N_{A B}^{P}$ is the differential yield of a point-like process $P$ in a $A+B$ collision and $d \sigma_{N N}^{P}$ is the cross section of $P$ in $N+N$ collision. If there are no initialor final-state effects that modify the yield of $P$ in $A+B$ collisions, the process $P$ scales with $\left\langle T_{A B}\right\rangle_{f}$ and $R_{A B}=1$. Sometimes, the central to peripheral ratio, $R_{C P}$, is used as an alternative to $R_{A B}$. The central to peripheral ratio is defined as

$$
R_{C P}=\frac{d N^{\text {Central }} /\left\langle N_{\text {coll }}^{\text {Central }}\right\rangle}{d N^{\text {Peripheral }} /\left\langle N_{\text {coll }}^{\text {Peripheral }}\right\rangle},
$$

where $d N^{\text {Central }}$ and $d N^{\text {Peripheral }}$ are the differential yield per event of the studied process in a central and peripheral collision, respectively. If the yield of the process scales with the number of binary collisions, $R_{C P}=1$.

\subsection{Binary Scaling in $l+A, p+A$, and Low-Energy $A+A$}

In deeply inelastic lepton scattering, where hard scattering was discovered $[153,154,155]$, the cross section for $\mu$-A collisions is indeed proportional to $A^{1.00}$ (Fig. 28). This indicates that the structure function of a nucleus of mass 


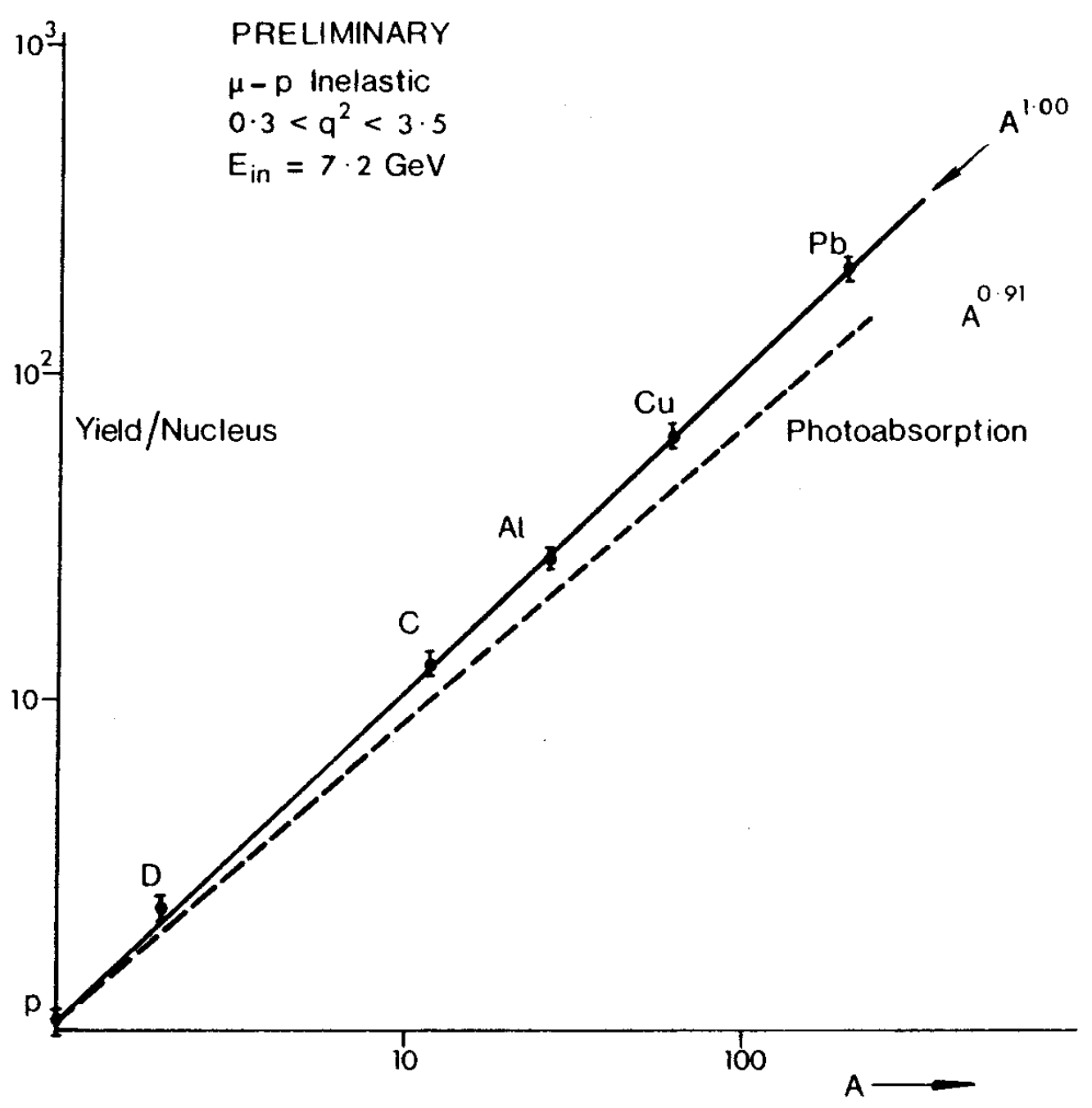

Fig. 28. $\mu$ - $A$ cross section vs. $A$ [156].

$A$ is simply $A$ times the structure function of a nucleon (with only minor deviations, $\leq 10 \%$ for $0.02 \leq x \leq 0.50$ [157]), which means that the nucleus acts like an incoherent superposition of nucleons for hard scattering of leptons.

The situation is rather different in $p+A$ collisions: the cross section at a given $p_{T}$ also scales as a power law, $A^{\alpha\left(p_{T}\right)}$ (Fig. 29), but the power $\alpha\left(p_{T}\right)$ is greater than 1. This is called the "Cronin Effect" [158]. The enhancement (relative to $A^{1.00}$ ) is thought to be due to the multiple scattering of the incident partons while passing through the nucleus $A$ before the collision $[159,160]$, which smears the axis of the hard scattering relative to the axis of the incident beam, leading to the characteristic "Cronin Effect" shape for $R_{A}\left(p_{T}\right)$ (Fig. 30). At low $p_{T}<1 \mathrm{GeV} / c$, the cross-section is no longer point like, so the scattering is shadowed $\left(\propto A^{2 / 3}\right)$, thus $R_{A}<1$. At larger $p_{T}>2 \mathrm{GeV} / c$, as the hard-scattering, power-law $p_{T}$ spectrum begins to dominate, the multiple scattering smears the spectrum to larger $p_{T}$ leading to an enhancement relative 


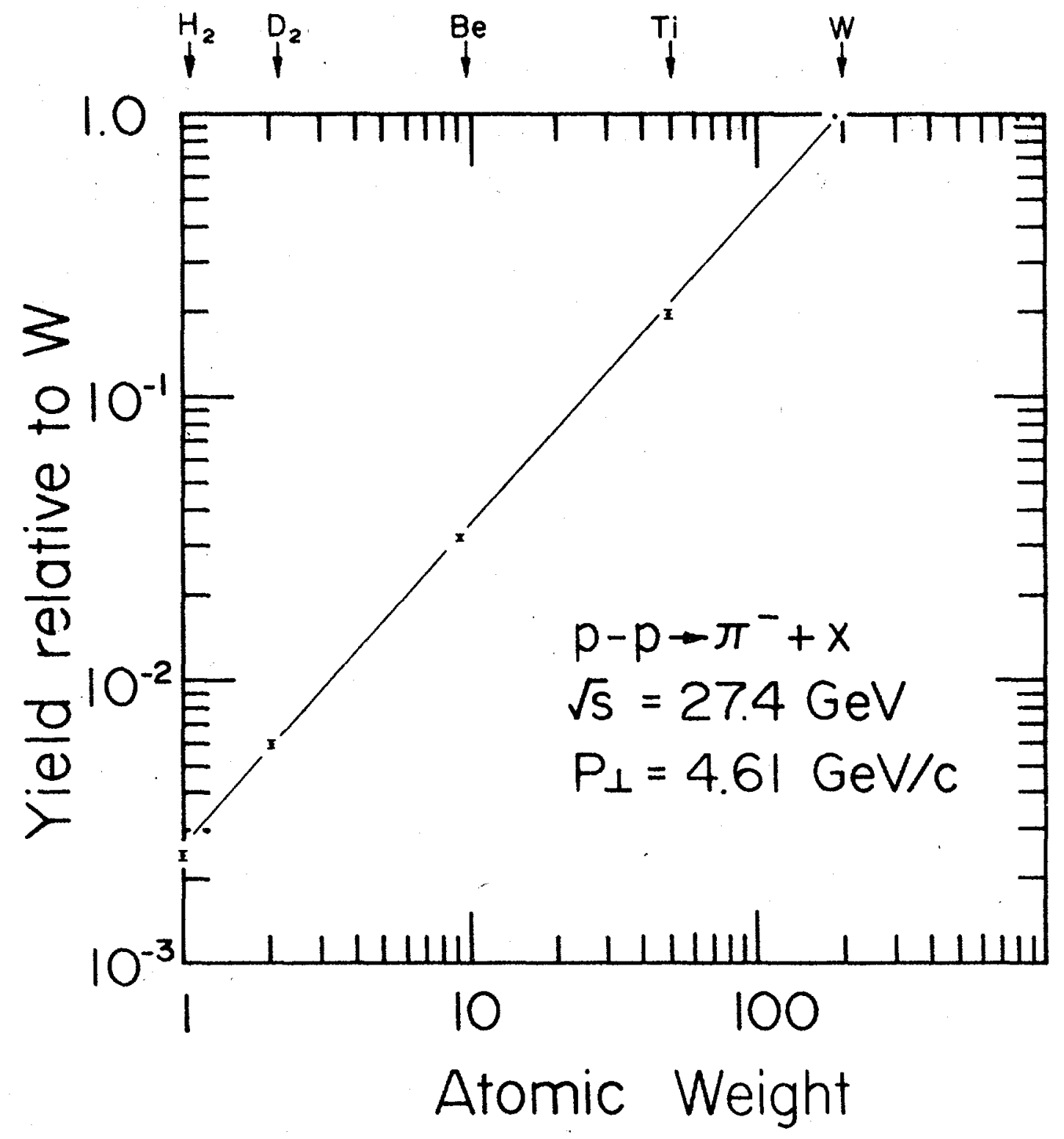

Fig. 29. Cronin Effect in $p+A$, for $\pi^{-}$with $p_{T}=4.61 \mathrm{GeV} / c . \alpha\left(p_{T}\right)=1.148 \pm 0.010$ [158].

to binary-scaling which dissipates with increasing $p_{T}$ as the influence of the multiple scattering diminishes.

Previous measurements of high- $p_{T}$ particle production in $A+A$ collisions at $\sqrt{s_{N N}} \leq 31 \mathrm{GeV}$ (Fig. 31) and in $p+A$ (or $d+A$ ) collisions (Fig. 30) including measurements at RHIC [64] at mid-rapidity (Fig. 32) all show binary scaling or a Cronin effect.

This establishes that the initial condition for hard scattering at RHIC at mid-rapidity is an incoherent superposition of nucleon structure functions, including gluons, where multiple scattering before the hard collision smears the $p_{T}$ spectrum of scattered particles to be somewhat above the simple pointlike binary $\left(N_{\text {coll }}\right)$ scaling. 


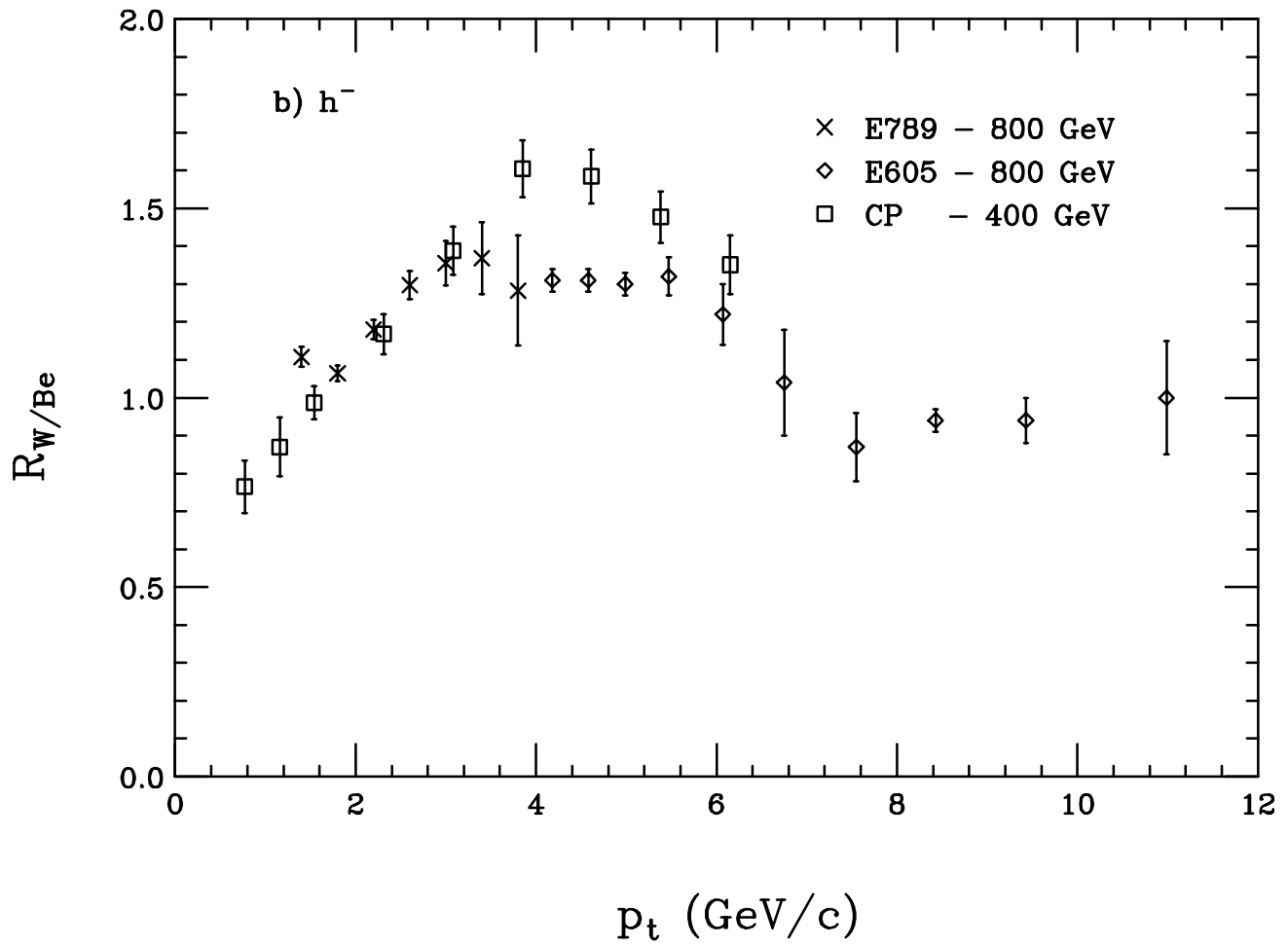

Fig. 30. Cronin effect at fixed target energies expressed as $R_{\mathrm{W} / \mathrm{Be}}$, the ratio of the point-like scaled cross sections in $p+W$ and $p+B e$ collisions vs. $p_{T}$ [161].

An alternative view of the initial state of a nucleus at RHIC is provided by the color glass condensate (CGC), in which the gluon population at low $x$ is not an incoherent superposition of nucleon structure functions but is limited with increasing $A$ by non-linear gluon-gluon fusion resulting from the overlap of gluons from several nucleons in the plane of the nucleus transverse to the collision axis [168]. A Cronin effect in $d+A$ collisions, as shown in Fig. 32, can be reproduced in the $\mathrm{CGC}$ with a suitable choice of initial state parameters, which must also reproduce quantitatively the observed binary scaling of the direct photon production and total charm production in $\mathrm{Au}+\mathrm{Au}$ collisions to be shown below (Figs. 33, 34). However, at this writing, no detailed quantitative description of the CGC initial state which satisfies these three conditions has been published.

\subsection{Binary Scaling in Au+Au Collisions at RHIC-Direct Photons and Charm Yield}

The production of hard photons in $\mathrm{Au}+\mathrm{Au}$ collisions at $\mathrm{RHIC}$ via the constituent reactions (e.g. $g+q \rightarrow \gamma+q$ ) is a very important test of QCD and the initial state, because the photons only interact electromagnetically, hence 


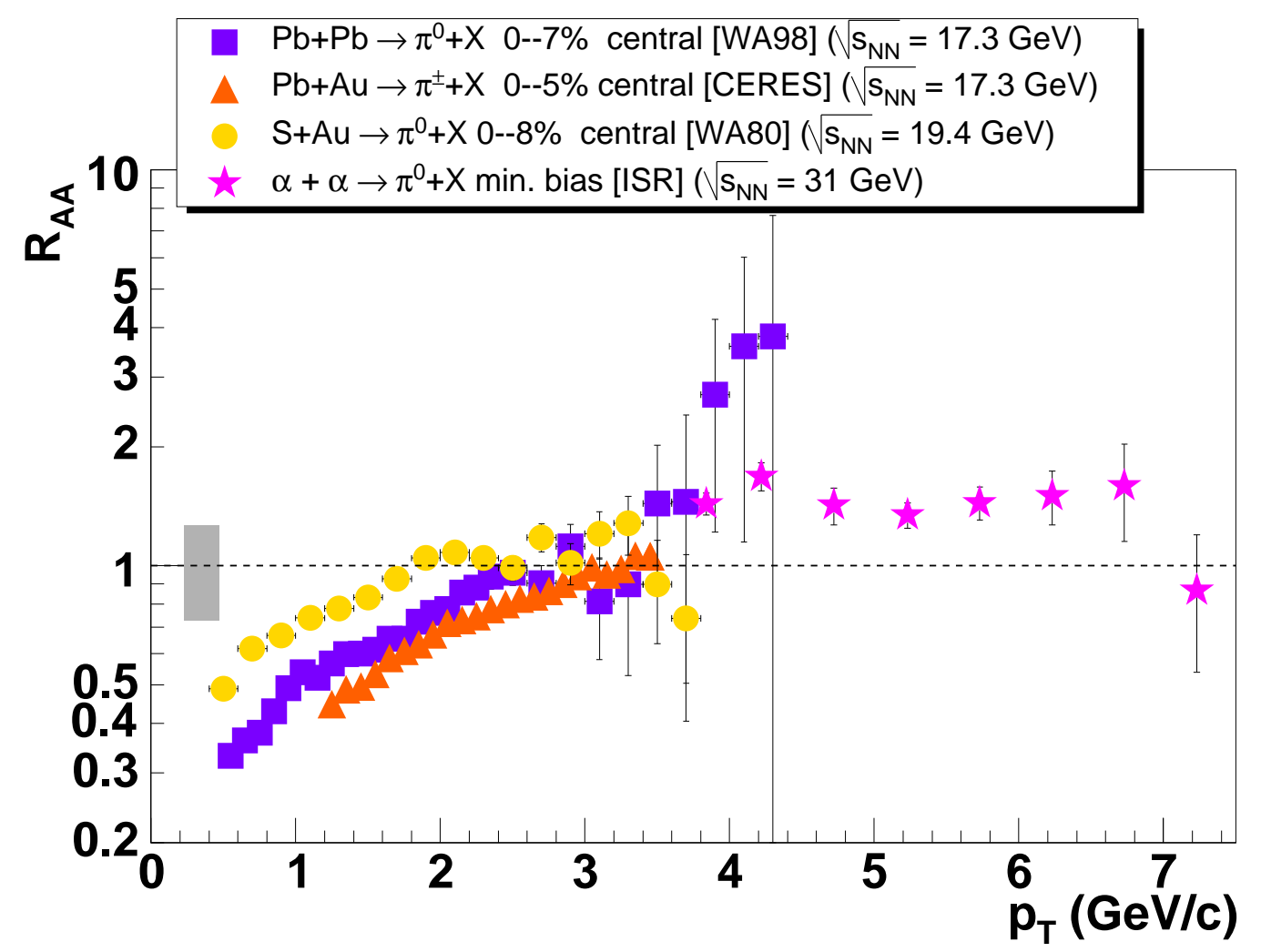

Fig. 31. Nuclear modification factors for $\pi^{0}$ production at the CERN-ISR in minimum-bias $\alpha+\alpha$ reactions at $\sqrt{s_{N N}}=31 \mathrm{GeV}$ [162] and for pion production at the CERN-SPS in central $\mathrm{Pb}+\mathrm{Pb}$ [163], $\mathrm{Pb}+\mathrm{Au}$ [164], and $\mathrm{S}+\mathrm{Au}$ [165] reactions at $\sqrt{s_{N N}} \approx 20 \mathrm{GeV}$. The $R_{A A}$ from SPS are obtained using the $p+p$ parametrization proposed in ref. [166]. The shaded band around $R_{A A}=1$ represents the overall fractional uncertainty of the SPS data (including in quadrature the $25 \%$ uncertainty of the $p+p$ reference and the $10 \%$ error of the Glauber calculation of $N_{\text {coll }}$ ). There is an additional overall uncertainty of $\pm 15 \%$ for the CERES data not shown in the plot [164].

hardly at all, with any final-state medium produced. The direct-photon cross section and centrality dependence should then reflect only the properties of the initial state, notably the product of the gluon and quark structure functions of the Au nuclei.

The first measurement of direct photon production in $\mathrm{Au}+\mathrm{Au}$ collisions at RHIC has been reported by the PHENIX collaboration (Fig. 33) [73]. The data exhibit pure point-like $\left(N_{\text {coll }}\right)$ scaling as a function of centrality relative to a pQCD calculation for $p+p$ collisions. The statistical and systematic errors still leave some room for a small Cronin effect and/or some thermal photon production. The observation of direct photon production establishes the importance of gluon degrees of freedom at RHIC.

PHENIX measured the single-electron yield from nonphotonic sources in $\mathrm{Au}+\mathrm{Au}$ 


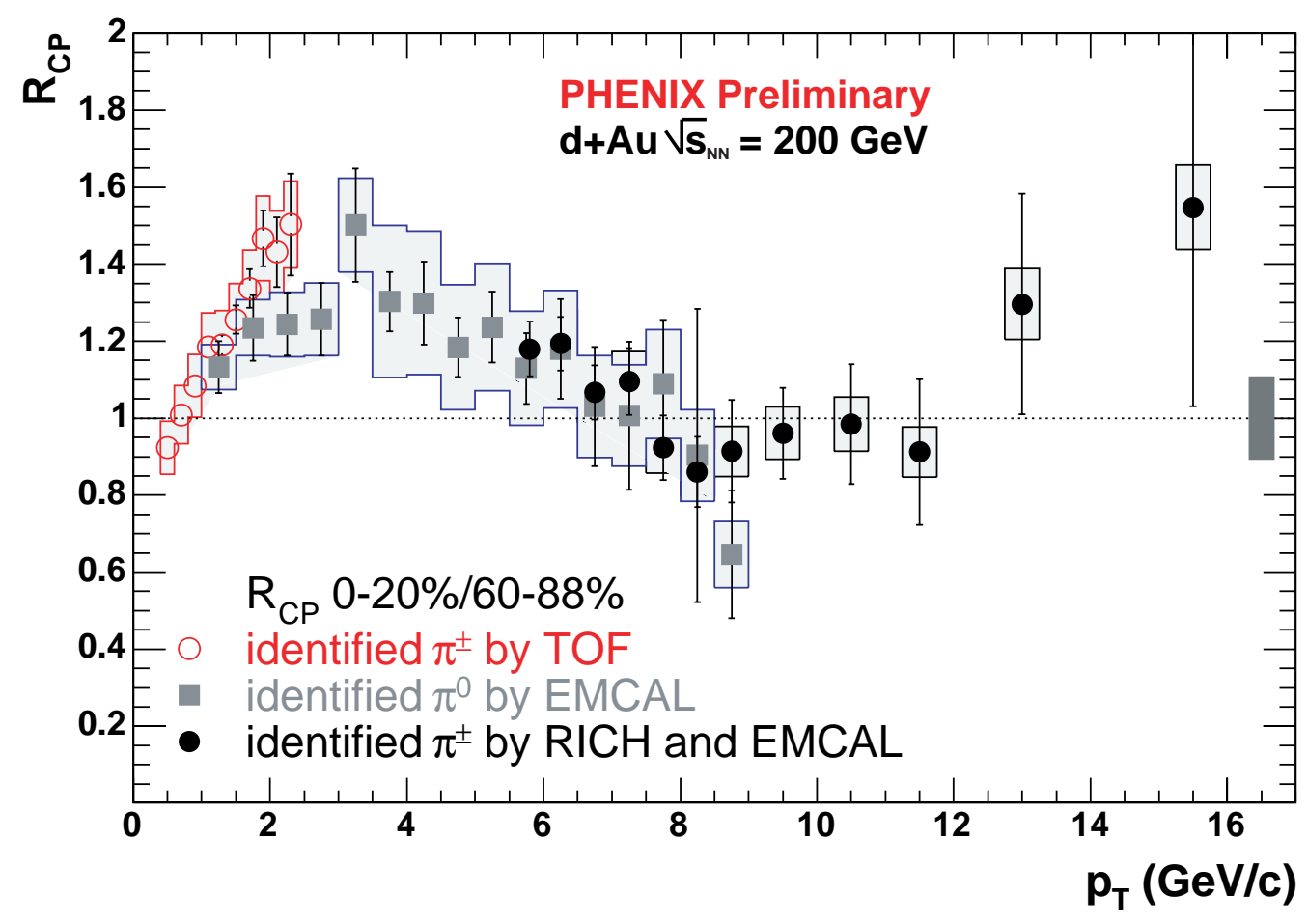

Fig. 32. Cronin effect in $R_{C P}$, the ratio of point-like scaled central to peripheral collisions for pions in $d+\mathrm{Au}$ at $\sqrt{s_{N N}}=200 \mathrm{GeV}$ [167]. Data points for low $p_{T}$ are $\pi^{ \pm}$identified by Time of Flight (TOF). Data at medium $p_{T}$ are for $\pi^{0}$ identified by reconstruction in the Electromagnetic Calorimeter (EMCAL). Highest $p_{T}$ data are for $\pi^{ \pm}$identified by a count in the Ring Imaging Cerenkov Counter (RICH) and a deposited energy/momentum and shower shape in the EMCAL inconsistent with those of a photon or electron. The shaded band on the right represents the overall fractional systematic uncertainty due to $N_{\text {coll }}$.

collision at $130 \mathrm{GeV}$ [42] and $200 \mathrm{GeV}$ [57]. Since semi-leptonic decay of charm is the dominant source of the non-photonic electrons at low $p_{T}\left(p_{T} \leq 3\right.$ $\mathrm{GeV} / c)$, the total yield of charm can be determined from the integrated yield of non-photonic electrons in the low- $p_{T}$ region. Figure 34 shows the yield of non-photonic electrons $\left(0.8<p_{T}<4.0 \mathrm{GeV} / c\right)$ per $N N$ collision in $\mathrm{Au}+\mathrm{Au}$ reactions at $\sqrt{s_{N N}}=200 \mathrm{GeV}$ as a function of $N_{\text {coll }}$ [57]. The $N_{\text {coll }}$ dependence of the yield is fit to $N_{\text {coll }}^{\alpha}$, where $\alpha=1$ is the expectation for binary scaling. We find $\alpha=0.938 \pm 0.075$ (stat.) \pm 0.018 (sys.), showing that the total yield of charm-decay electrons is consistent with binary scaling. It should be noted that medium effects, such as energy loss of charm in the dense hot medium, can only influence the momentum distribution of charm, and have little effect on the total yield of charm. Initial-state effects, such as shadowing, and other effects, such as thermal production of charm, are believed to be very small for charm production at RHIC energy. Therefore, the observation of binary scaling of the total charm yield in $\mathrm{Au}+\mathrm{Au}$ collisions at RHIC may also be considered as an experimental verification of the binary scaling of a point-like 


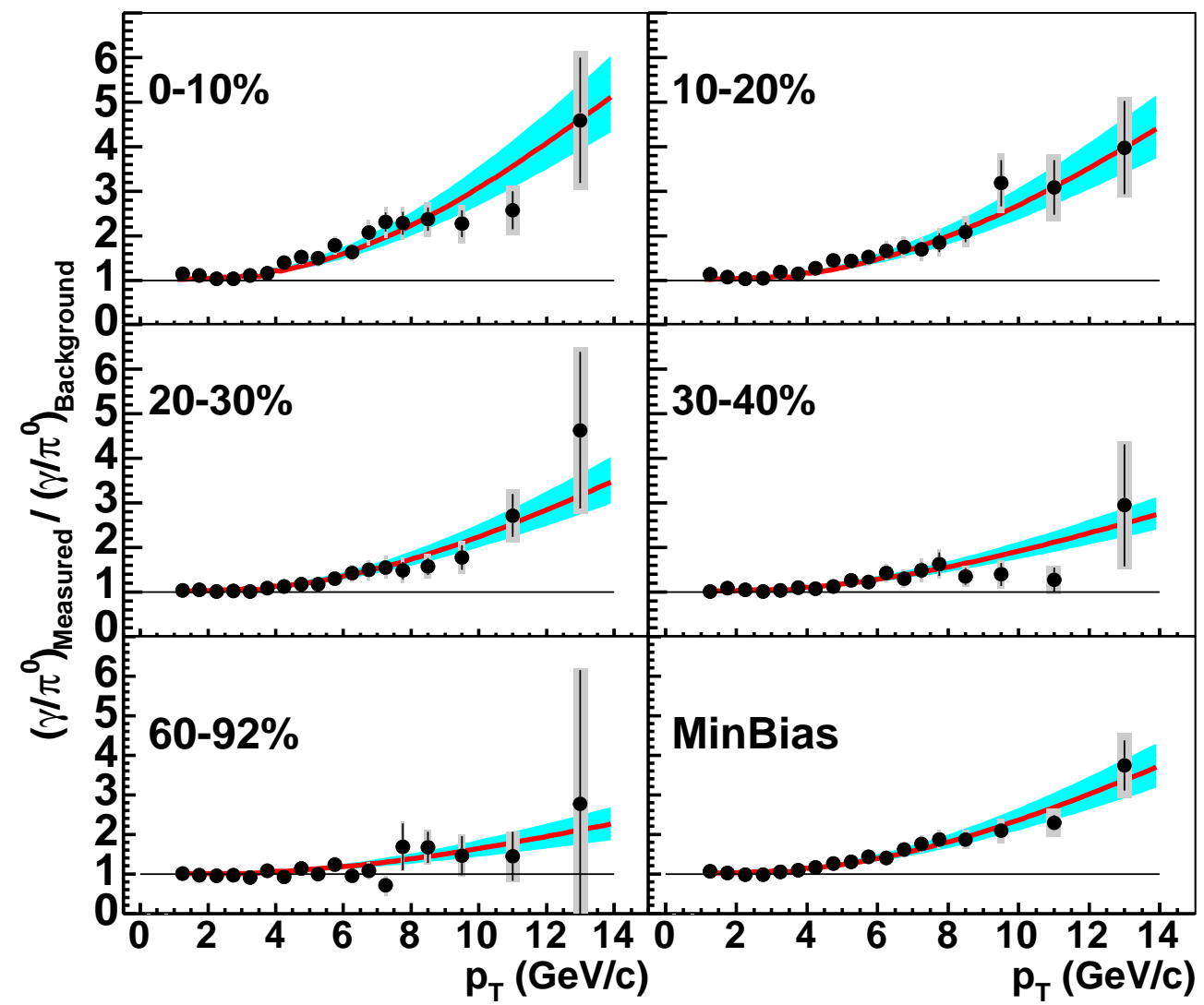

Fig. 33. PHENIX direct photon measurements relative to the background for for minimum bias and for five centralities of $\mathrm{Au}+\mathrm{Au}$ collisions at $\sqrt{s_{N N}}=200 \mathrm{GeV}$ (0-10\% is the most central). Statistical and total errors are indicated separately on each data point by the vertical bar and shaded region, respectively. The curves represent a pQCD calculation of direct photons in $p+p$ collisions from Vogelsang $[169,170,171,172]$ scaled to $\mathrm{Au}+\mathrm{Au}$ assuming pure point-like $\left(N_{\text {coll }}\right)$ scaling, with no suppression. The shaded region around the curves indicate the variation of the pQCD calculation for scale changes from $p_{T} / 2$ to $2 p_{T}$, plus the $\left\langle N_{\text {coll }}\right\rangle$ uncertainty [73].

pQCD process.

\subsection{Conclusions}

In this section evidence has been presented to show that the initial condition for hard-scattering at RHIC at mid-rapidity is an incoherent superposition of nucleon structure functions, including gluons, where multiple scattering before the hard collision can smear the $p_{T}$ spectrum of scattered particles to be somewhat above the simple point-like binary $\left(N_{\text {coll }}\right)$ scaling. This was demonstrated using the reactions: pion production in $d+\mathrm{Au}$ collisions, where there is no final-state medium, and direct photon production in $\mathrm{Au}+\mathrm{Au}$ collisions, 


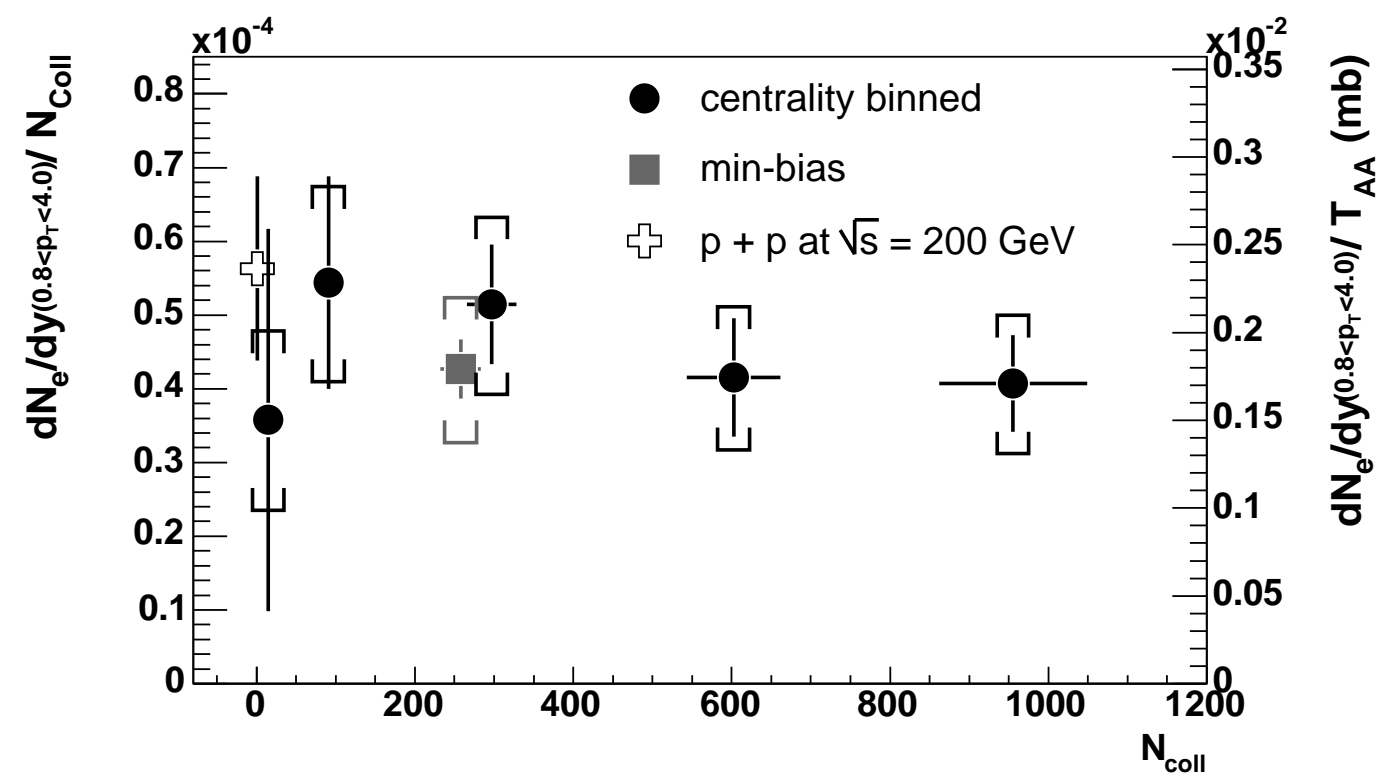

Fig. 34. Non-photonic electron yield $\left(0.8<p_{T}<4.0 \mathrm{GeV} / c\right)$, dominated by semi-leptonic charm decays, measured in $\mathrm{Au}+\mathrm{Au}$ collisions at $\sqrt{s_{N N}}=200 \mathrm{GeV}$ scaled by $N_{\text {coll }}$ as a function of $N_{\text {coll }}$. The right-hand scale shows the corresponding electron cross section per $N N$ collision in the above $p_{T}$ range. The yield in $p+p$ collision at $200 \mathrm{GeV}$ is also shown [57].

where the outgoing photons interact electromagnetically, hence hardly at all, with any final-state medium produced. The total charm yield in $\mathrm{Au}+\mathrm{Au}$, a reaction dominated by the subprocess $g+g \rightarrow c+\bar{c}$, and which is not sensitive to final-state medium effects for the total yield of $c+\bar{c}$ pairs, also exhibits binary scaling. The latter two measurements provide experimental evidence for the binary scaling of point-like pQCD processes in $\mathrm{Au}+\mathrm{Au}$ collisions.

The color glass condensate (CGC) provides an alternative view of the initial state of a nucleus at RHIC in which coherence of gluons due to non-linear gluon-gluon fusion can produce a Cronin-like effect, depending on the initial conditions and the kinematic range covered. However, at the present writing, there is no CGC description of the initial state nuclear structure function which reproduces the observed Cronin effect for pions in $d+\mathrm{Au}$ collisions and the observed binary scaling for both direct photon production and the total charm yield in $\mathrm{Au}+\mathrm{Au}$ collisions.

\section{NUMBER DENSITY AND HIGH $p_{T}$ SUPPRESSION}

To study the initial properties of the matter created in heavy ion collisions we need a probe that is already present at earliest times and that is directly sensitive to the properties of the medium. Partons resulting from hard scatterings during the initial crossing of the two nuclei in $\mathrm{A}+\mathrm{A}$ collisions provide 
such a probe. Energetic partons propagating through a dense medium are predicted to lose energy [173,174,175,176,177,178,179,180,181] thus producing a suppression in the yield of high- $p_{T}$ hadrons produced from the fragmentation of these partons. Initial measurements from RHIC Run $1[39,47,182]$ and Run $2[49,53,183,184]$ demonstrated such a suppression, and the results of $d+$ Au measurements $[64,68,67,66]$ showed that the suppression was not due to initial-state effects. Further measurements have indicated a modification of di-jet angular correlations [68] that has also been attributed to in-medium parton energy loss $[185,186]$.

While the energy loss of hard-scattered partons was originally proposed as a signature of the quark-gluon plasma and deconfinement, it has been argued recently that the energy loss is sensitive only to the density of unscreened color charges and not directly to deconfinement $[177,178,179,180,181,187,188]$. Ideally, a measurement of initial parton densities together with constraints on initial energy density might allow an estimate of the temperature of the medium. As will be seen below, the current high- $p_{T}$ measurements and theoretical tools for interpreting the experimental data are not yet sufficient to take such a step. Instead, the energy loss results are currently being used to provide estimates of the initial energy density. The remainder of this section summarizes PHENIX experimental data related to high- $p_{T}$ suppression, discusses the current state of theoretical understanding of the energy loss process and concludes with a statement of estimates for initial parton number and energy densities that currently can be made.

\subsection{Single particle spectra, $R_{A A}$}

As described in Section 5, in the absence of modifications due to initial-state or final-state effects, the rate for the production of particles through hardscattering processes in nucleus-nucleus collisions is expected to be given by the equivalent $p+p$ hard-scattering cross section multiplied by $T_{A B}$. Figure 35 shows PHENIX $\pi^{0}$ spectra, $d^{2} N / d p_{T} d y$, measured in $200 \mathrm{GeV}$ [49] peripheral (80-92\%) and central (0-10\%) Au+Au collisions compared to measured [60] $p+p$ cross sections multiplied by the peripheral and central $T_{A B}$ values estimated using the procedure described in Section 5. The error bands on the $p+p$ data points reflect both the systematic errors on the $p+p$ cross sections and the uncertainties in the $T_{A B}$ values. As the figure clearly demonstrates, the central $\mathrm{Au}+\mathrm{Au} \pi^{0}$ yields are strongly suppressed relative to the "expected" yields over the entire measured $p_{T}$ range. In contrast, the peripheral yields compared to the $T_{A B}$-scaled $p+p$ cross sections show little or no suppression. The results incontrovertibly demonstrate that there is a strong and centralitydependent suppression of the production of high- $p_{T}$ pions relative to pQCD-

motivated expectations. This is quite different from measurements of $R_{A A}$ 
in $\mathrm{Pb}+\mathrm{Pb}$ collisions at $\sqrt{s_{N N}}=17.3 \mathrm{GeV}$ where in semi-peripheral $\mathrm{Pb}+\mathrm{Pb}$ collisions there is a nuclear enhancement increasing with $p_{T}$ similar to the well-known Cronin effect, while in central collisions the Cronin enhancement appears to be weaker than expected

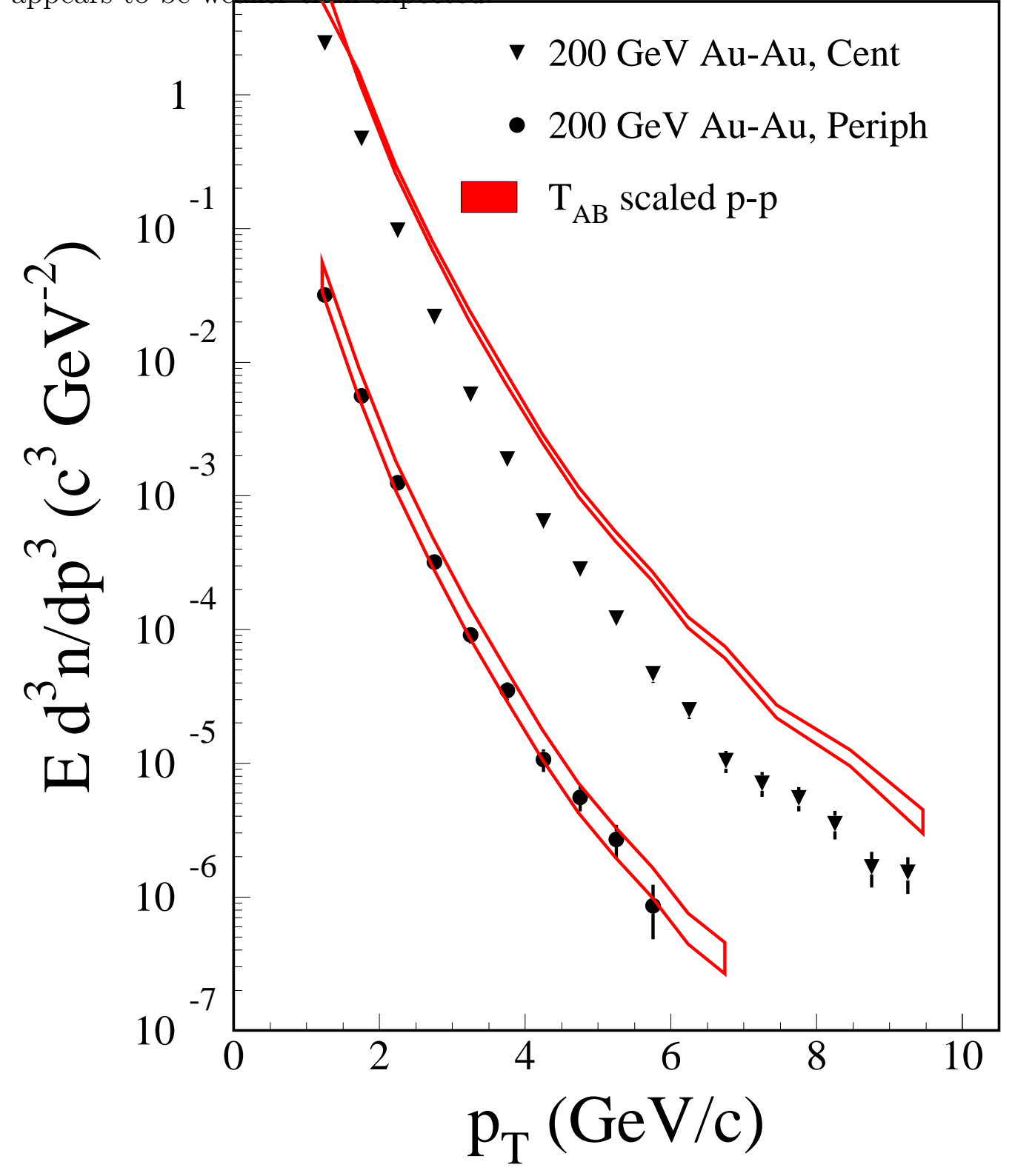

Fig. 35. $\pi^{0} p_{T}$ spectra in $200 \mathrm{GeV} \mathrm{Au+Au} \mathrm{collisions} \mathrm{[49]} \mathrm{compared} \mathrm{to} \mathrm{a} T_{A B}$ scaling of the $200 \mathrm{GeV} p+p \pi^{0}$ differential cross section [60]. The central data were obtained with a $0-10 \%$ centrality cut while the peripheral data were obtained with an $80-92 \%$ cut.

To better demonstrate quantitatively the suppression in central collisions indicated in Fig. 35, we show in Fig. $36 R_{A A}\left(p_{T}\right)$ for mid-rapidity $\pi^{0}$ 's in central and peripheral $200 \mathrm{GeV} \mathrm{Au}+\mathrm{Au}$ collisions. We also show the values obtained from minimum-bias $200 \mathrm{GeV} d+\mathrm{Au}$ collisions [64] which provide a stringent test of the possible contribution of initial-state nuclear effects to the observed 
suppression in $\mathrm{Au}+\mathrm{Au}$ collisions. The error bands on the data indicate combined statistical and point-to-point systematic errors and the bars shown next to the different data sets indicate common systematic errors due to uncertainties in the $p+p$ cross section normalization and $T_{A B}$.

Figure 36 shows that the central $\mathrm{Au}+\mathrm{Au} \pi^{0}$ suppression changes only slightly over the measured $p_{T}$ range and reaches an approximately $p_{T}$-independent factor of $5\left(R_{A A} \approx 0.2\right)$ for $p_{T}>4-5 \mathrm{GeV} / c$. The peripheral $\mathrm{Au}+\mathrm{Au} R_{A A}$ values are consistent with one after taking into account systematic errors but we cannot rule out a slight suppression suggested by the peripheral $R_{A A}$ values. In all of the data sets $R_{A A}$ decreases with decreasing $p_{T}$ for $p_{T}<2 \mathrm{GeV} / c$. This decrease, known since the original measurements of the $A$ dependence of particle production in $p A$ collisions is due to contributions of soft hadronic processes at low $p_{T}$ that are expected to increase more slowly than proportional to $T_{A B}$. The $d+\mathrm{Au} R_{d A}$ values are also consistent with one within systematic uncertainties, but in contrast to the $\mathrm{Au}+\mathrm{Au}$ results, the data suggest a slight enhancement. The $d+\mathrm{Au} R_{d A}$ values above $2 \mathrm{GeV} / c$ exceed one for nearly the entire experimentally covered $p_{T}$ range. As shown previously in Fig. 32, only for $p_{T} \gtrsim 6 \mathrm{GeV} / c$ does the $d+\mathrm{Au}$ pion yield return to the $T_{A B}$-scaling expectation. Such a small enhancement is consistent with expectations based on prior measurements of the Cronin effect $[189,190]$, and it is also quantitatively consistent with calculations incorporating the initial-state multiple scattering that is thought to produce the Cronin effect [191,192,193,194,195,196,197]. Therefore the Cronin effect at RHIC cannot mask a strong initial-state suppression of the parton distributions in the Au nucleus [198].

To better demonstrate the systematic behavior of the high- $p_{T}$ suppression we show in Fig. $37 \pi^{0}$ [49] and unidentified charged particle $R_{A A}$ values [53] as a function of $p_{T}$ for various centrality bins. While for moderate $p_{T}$ values $\left(2<p_{T}<5 \mathrm{GeV} / c\right)$ total charged particle production is suppressed less than pion production, the charged particle and $\pi^{0} R_{A A}$ values become equal, within errors, at high $p_{T}$. This evolution in the charged particle suppression is related to contributions from the (anti)protons that will be discussed further below. Despite the differences resulting from the protons, the charged particles and $\pi^{0}$ 's exhibit very similar trends in the suppression vs. $p_{T}$ and vs. centrality. The suppression increases smoothly with centrality though the change in $R_{A A}$ values at high $p_{T}$ is most rapid in the middle of the centrality range. Figure 37 also shows that the suppression is approximately constant as a function of $p_{T}$ for $p_{T}>4.5 \mathrm{GeV} / c$ in all centrality bins. We take advantage of this feature of the data to better illustrate the centrality dependence of the suppression by integrating both the $\mathrm{Au}+\mathrm{Au}$ spectra and the reference $p+p$ cross sections over $p_{T}>4.5 \mathrm{GeV} / c$ and using these integrated quantities to determine an average suppression factor, $R_{A A}$ for $p_{T}>4.5 \mathrm{GeV} / c$. We plot the charged particle and $\pi^{0} R_{A A}$ values vs. $N_{\text {part }}$ in Fig. 38(top). This figure suggests that the suppression evolves smoothly with $N_{\text {part }}$, showing no abrupt 


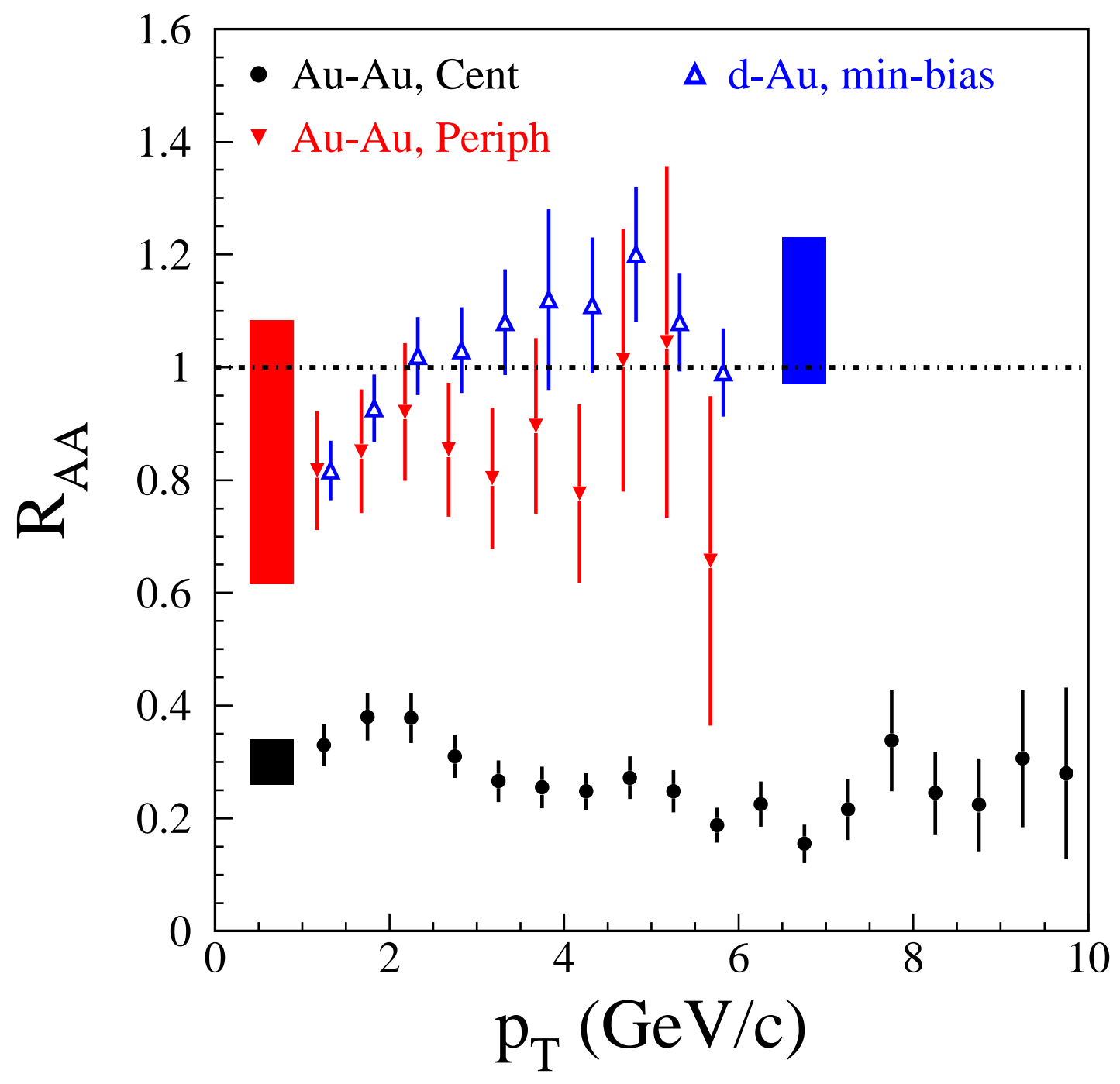

Fig. 36. $\pi^{0} R_{A A}\left(p_{T}\right)$ for central (0-10\%) and peripheral (80-92 \%) Au+Au collisions [49] and minimum-bias $d+\mathrm{Au}$ collisions [64]. The shaded boxes on the left show the systematic errors for the $\mathrm{Au}+\mathrm{Au} R_{A A}$ values resulting from overall normalization of spectra and uncertainties in $T_{A B}$. The shaded box on the right shows the same systematic error for the $d+\mathrm{Au}$ points.

onset of suppression. The charged particles and $\pi^{0}$ 's exhibit similar evolution of suppression with $N_{\text {part }}$. In the most central collisions we obtain $R_{A A}$ values of $0.24 \pm 0.04$ (total) and $0.23 \pm 0.05$ (total) for charged particles and $\pi^{0}$ 's respectively. In peripheral collisions, $R_{A A}$ approaches one, but the systematic errors on the most peripheral $T_{A B}$ values are sufficiently large that we cannot rule out $\sim 20 \%$ deviations of the peripheral $\mathrm{Au}+\mathrm{Au}$ hard-scattering yields from the $T_{A B}$-scaled $p+p$ cross sections.

An alternative method for evaluating the evolution of the high- $p_{T}$ suppression 

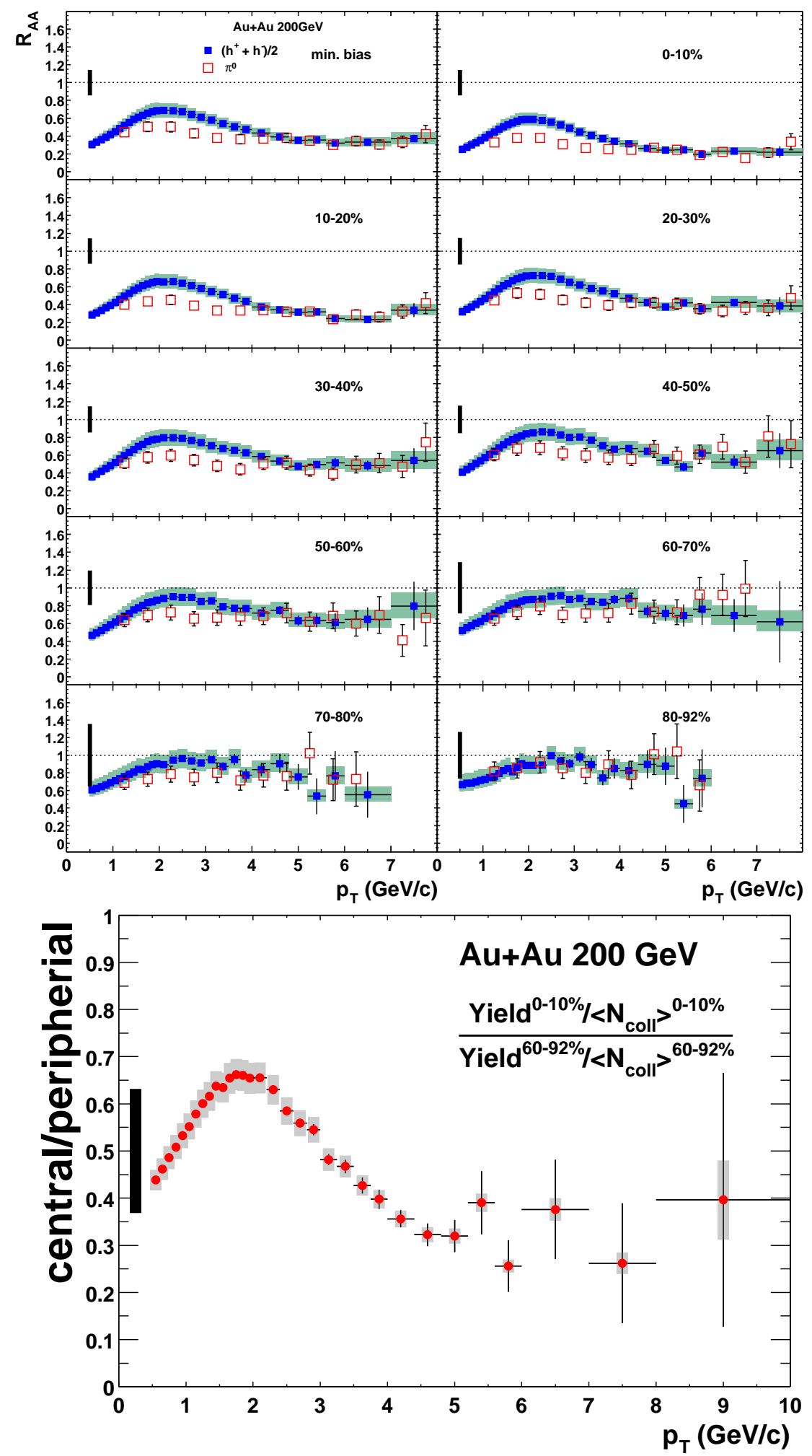

Fig. 37. Centrality and $p_{T}$ dependence of nuclear modification factors in $200 \mathrm{GeV}$ $\mathrm{Au}+\mathrm{Au}$ collisions[53]. Top panel: $\pi^{0}$ and charged particle $R_{A A}\left(p_{T}\right)$ for ten centrality bins. Bottom panel: charged particle $R_{C P}$ vs. $p_{T}$. 

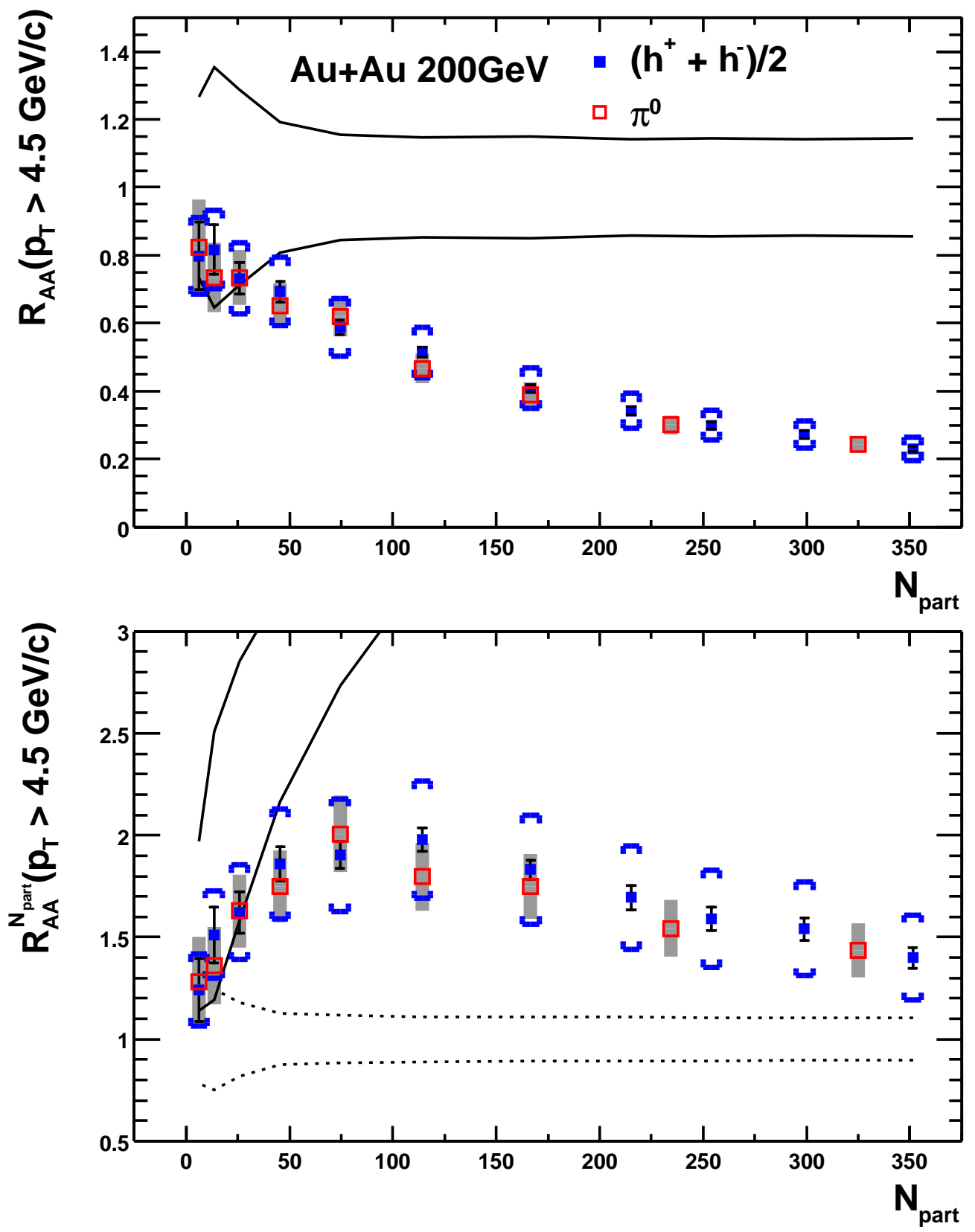

Fig. 38. Top panel: $R_{A A}$ vs. $N_{\text {part }}$ obtained from $p_{T}$-integrated $\left(p_{T}>4.5 \mathrm{GeV} / c\right)$ $\mathrm{Au}+\mathrm{Au} \pi^{0}$ and charged-hadron spectra. The band indicates the systematic error bands on a hypothetical $T_{A B}$ scaling of the $p+p p_{T}$-integrated cross section. Bottom panel: $\pi^{0}$ and charged hadron yield per participant vs. $N_{\text {part }}$ divided by the same quantity in $p+p$ collisions $\left(R_{A A}^{N_{\text {part }}}\right)$. The solid band shows the same band as in the top panel expressed in terms of yield per participant pair while the dashed band indicates the systematic error bands around a hypothetical $N_{\text {part }}$ scaling. Both plots are from [53]. 
with centrality is provided in Fig. 38(bottom) which presents the charged and $\pi^{0}$ yields per participant integrated over $p_{T}>4.5 \mathrm{GeV} / c$ as a function of $N_{\text {part }}$ [53] divided by the same quantity in $p+p$ collisions. Also shown in the figure are curves demonstrating the $N_{\text {part }}$ dependence that would result if the $\pi^{0}$ and charged particle yields exactly $T_{A B}$ scaled and what an $N_{\text {part }}$ scaling from $p+p$ collisions would imply. As Fig. 38 demonstrates, the high- $p_{T}$ yields of both charged hadrons and $\pi^{0}$ 's per participant increase proportional to $T_{A B}$ for small $N_{\text {part }}$ but level off and then decrease with increasing $N_{\text {part }}$ in more central collisions. The PHENIX measurements do not naturally support an approximate $N_{\text {part }}$ scaling of high- $p_{T}$ particle production suggested in an analysis of PHOBOS data. The PHENIX $R_{A A}^{N_{\text {part }}}$ values decrease from mid-peripheral $\left(N_{\text {part }} \approx 75\right)$ to central collisions by an amount larger than the systematic errors in the measurement. For more peripheral collisions, $R_{A A}^{N_{\text {part }}}$ increases with $N_{\text {part }}$ consistent with the modest suppression of high- $p_{T}$ production shown for peripheral collisions in the top panel of Fig. 38. The initial rise and subsequent decrease of $R_{A A}^{N_{\text {part }}}$ with increasing $N_{\text {part }}$ suggests that the high- $p_{T}$ hadron yield in $\mathrm{Au}+\mathrm{Au}$ collisions has no simple dependence on $N_{\text {part }}$. The observation that the high- $p_{T}$ yields initially increase proportional to $T_{A B}$ demonstrates that in the most peripheral $\mathrm{Au}+\mathrm{Au}$ collisions the hard-scattering yields are consistent with point-like scaling. However, the deviation from $T_{A B}$ scaling sets in rapidly, becoming significant by $N_{\text {part }}=50$. By $N_{\text {part }}=100$ the high- $p_{T}$ suppression is so strong that high- $p_{T}$ yields grow even more slowly than proportional to $N_{\text {part }}$.

\section{2 $x_{T}$ scaling in $A u+A u$ collisions at $R H I C$}

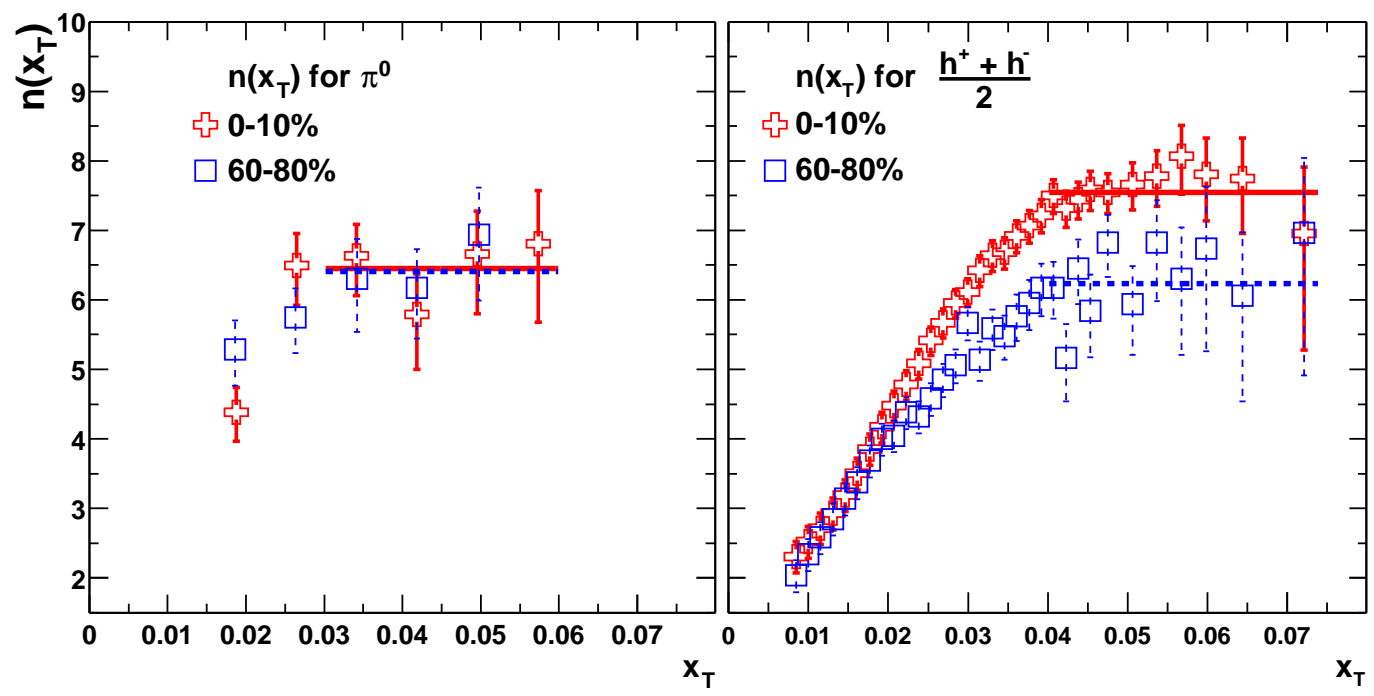

Fig. 39. Power-law exponent $n\left(x_{T}\right)$ for $\pi^{0}$ and $h$ spectra in central and peripheral $\mathrm{Au}+\mathrm{Au}$ collisions at $\sqrt{s_{N N}}=130$ and $200 \mathrm{GeV}[53]$. 
If the production of high- $p_{T}$ particles in $\mathrm{Au}+\mathrm{Au}$ collisions is the result of hard scattering according to pQCD, then $x_{T}$ scaling should work just as well in $\mathrm{Au}+\mathrm{Au}$ collisions as in $p+p$ collisions and should yield the same value of the exponent $n\left(x_{T}, \sqrt{s}\right)$. The only assumption required is that the structure and fragmentation functions in $\mathrm{Au}+\mathrm{Au}$ collisions should scale, in which case Eq. 23 still applies, albeit with a $G\left(x_{T}\right)$ appropriate for $\mathrm{Au}+\mathrm{Au}$. In Fig. 39, $n\left(x_{T}, \sqrt{s_{N N}}\right)$ in $\mathrm{Au}+\mathrm{Au}$ is derived from Eq. 23, for peripheral and central collisions, by taking the ratio of $E d^{3} \sigma / d p^{3}$ at a given $x_{T}$ for $\sqrt{s_{N N}}=130$ and $200 \mathrm{GeV}$, in each case. The $\pi^{0}$ 's exhibit $x_{T}$ scaling, with the same value of $n=6.3$ as in $p+p$ collisions, for both $\mathrm{Au}+\mathrm{Au}$ peripheral and central collisions, while the non-identified charged hadrons $x_{T}$-scale with $n=6.3$ for peripheral collisions only. Notably, the $h^{ \pm}$in $\mathrm{Au}+\mathrm{Au}$ central collisions exhibit a significantly larger value of $n$, indicating different physics, which will be discussed below. The $x_{T}$ scaling establishes that high- $p_{T} \pi^{0}$ production in peripheral and central $\mathrm{Au}+\mathrm{Au}$ collisions and $h^{ \pm}$production in peripheral $\mathrm{Au}+\mathrm{Au}$ collisions follow pQCD as in $p+p$ collisions, with parton distributions and fragmentation functions that scale with $x_{T}$, at least within the experimental sensitivity of the data.

\subsection{Two-hadron azimuthal-angle correlations}

We argued in Sec. 5 that the production of hadrons at high- $p_{T}$ results predominantly from hard scattering followed by fragmentation of the outgoing parton(s). While this result is well established in $p(\bar{p})+p$ collisions, it might not be true in $\mathrm{Au}+\mathrm{Au}$ collisions when the yield of high- $p_{T}$ particles is modified so dramatically compared to expectations. Since a hard-scattered parton fragments into multiple particles within a restricted angular region (i.e. a jet) a reasonable way to check the assumption that high- $p_{T}$ hadron production in $\mathrm{Au}+\mathrm{Au}$ collisions is due to hard scattering is to directly observe the angular correlations between hadrons in the jets. None of the experiments at RHIC are currently capable of reconstructing jets in the presence of the large soft background of a Au+Au collision. However, both STAR [199,200] and PHENIX [201,202] have directly observed the presence of jets by studying two-hadron azimuthal-angle correlations. Figure 40 shows preliminary distributions [201] of the relative azimuthal angle $(\Delta \phi)$ between pairs of charged particles detected within the PHENIX acceptance in $d+\mathrm{Au}$ collisions and peripheral (60-90\%) and central (0-10\%) Au+Au collisions after the subtraction of combinatoric background. The pairs of particles are chosen such that one particle lies within a "trigger" $p_{T}$ range $\left(2.5<p_{T \text { trig }}<4 \mathrm{GeV} / c\right)$ while the other "associated" particle falls within a lower $p_{T}$ window $1.0<p_{T}<2.5$ $\mathrm{GeV} / c$. The distributions show the differential yield per $\Delta \phi$ of associated particles per detected trigger particle within the given $p_{T}$ ranges and within the $\eta$ acceptance of the PHENIX central arms $(-0.35<\eta<0.35)$. 
- Au-Au Central

- Au-Au Periph

$\checkmark$ d-Au Min-Bias

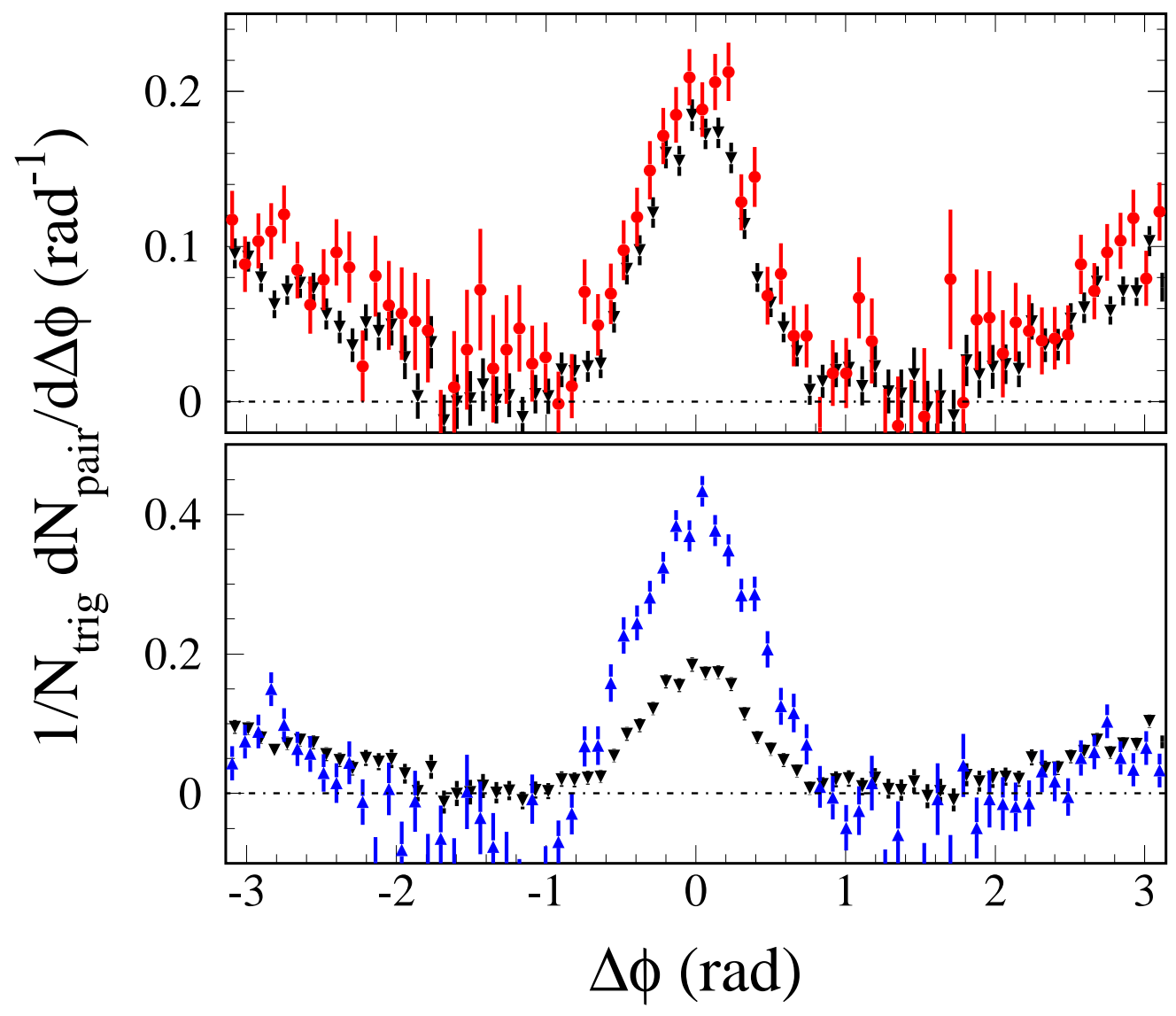

Fig. 40. Differential yields per $\Delta \phi$ and per trigger particle of pairs of charged hadrons in $d+\mathrm{Au}$, peripheral $\mathrm{Au}+\mathrm{Au}$ and central $\mathrm{Au}+\mathrm{Au}$ collisions at $\sqrt{s_{N N}}=200$ $\mathrm{GeV}[201]$. The pairs were selected with the higher-momentum "trigger" particle in the range $2.5<p_{T}<4.0 \mathrm{GeV} / c$ and the lower-momentum "associated" particle in the range $1.0<p_{T}<2.5 \mathrm{GeV} / c$. A constant background has been subtracted for all three distributions.

The peaks observed at $\Delta \phi=0$ (near side) reflect the correlation between hadrons produced within the same jet while the broader peaks observed at $\Delta \phi=\pi$ (away side) reflect the correlations between hadrons produced in one jet and hadrons produced in the "balance" jet. In the Au+Au cases, a $\cos 2 \Delta \phi$ modulation underlies the jet angular correlations due to the elliptic flow of particles in the combinatoric background and possibly also in part due to azimuthal anisotropies in the jets themselves (see below). Nonetheless, the $\cos 2 \Delta \phi$ contribution has little effect on the narrow same-jet (near-side) peak in the $\Delta \phi$ distribution.

We observe that the angular widths of the same-jet correlations are the same 
within errors in all three data sets in spite of the factor of two larger yield of associated hadrons in central $\mathrm{Au}+\mathrm{Au}$ collisions compared to $d+\mathrm{Au}$ and peripheral $\mathrm{Au}+\mathrm{Au}$ collisions. This result is demonstrated more quantitatively in Fig. 41 which shows the centrality dependence of the Gaussian widths of the same-jet peaks in the $\mathrm{Au}+\mathrm{Au} \Delta \phi$ compared to the jet widths extracted from $d+\mathrm{Au}$ collisions [201]. We see that the $\mathrm{Au}+\mathrm{Au}$ two-hadron correlation functions show peaks with the same jet width as $d+\mathrm{Au}$ collisions. Since this width is a unique characteristic of the parton fragmentation process, we conclude that high- $p_{T}$ hadrons in $\mathrm{Au}+\mathrm{Au}$ collisions result from hard scattering followed by jet fragmentation regardless of any medium modifications of the fragmentation multiplicity.

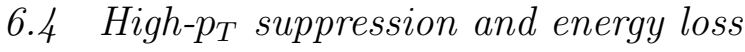

The suppression of the production of high- $p_{T}$ hadrons in heavy ion collisions at RHIC had been predicted long before RHIC started running [173,174,175,176,177,178,179,203]. It is now generally accepted that partons propagating in colored matter lose energy predominantly through medium-induced emission of gluon radiation [204,205]. An energetic parton scatters off color charges in the high-partondensity medium and radiates gluon bremsstrahlung. The reduction in the parton energy translates to a reduction in the average momentum of the fragmentation hadrons, which, in turn, produces a suppression in the yield of high- $p_{T}$ hadrons relative to the corresponding yield in $p+p$ collisions. The power-law spectrum for $p_{T} \geq 3 \mathrm{GeV} / c$ implies that a modest reduction in fragmenting parton energy can produce a significant decrease in the yield of hadrons at a given $p_{T}$. Thus, the suppression of the yield of high- $p_{T}$ hadrons is generally believed to provide a direct experimental probe of the density of color charges in the medium through which the parton passes $[206,188,194]$. However, before proceeding to an interpretation of our results, we briefly discuss the theoretical understanding of the radiative energy loss mechanism and limitations in that understanding.

The dominant role of radiative gluon emission was identified early on [175], but it took several years and much effort before rigorous calculations of the energy loss taking into account Landau-Pomeranchuk-Migdal suppression [176] and the time evolution of the medium were available. Initial estimates of the radiative energy loss suggested an approximately constant $\Delta E / \Delta x[174,176]$, but later calculations $[177,207,180,181]$ showed that the quantum interference can produce a loss of energy that grows faster than linearly with the propagation path length, $L$, of the parton in the medium. However, this ideal growth of $\Delta E / \Delta x$ with increasing path length is never realized in heavy ion collisions due to the rapid decrease of the energy density and the corresponding color charge density with time [179,188,194,185]. Generally, all energy loss calcula- 


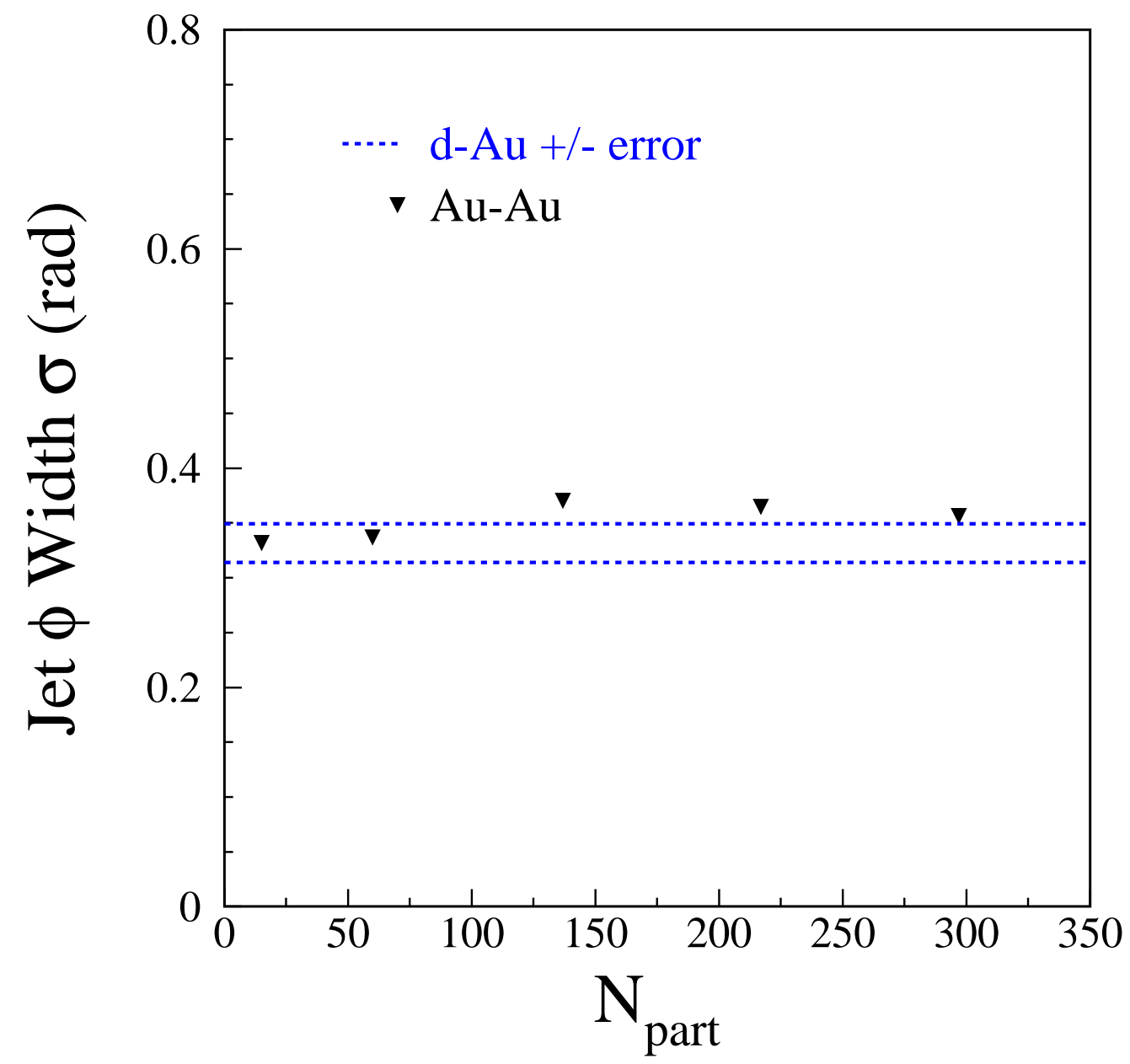

Fig. 41. The azimuthal angle width of jets in $200 \mathrm{GeV} \mathrm{Au+Au}$ collisions extracted as the $\sigma$ 's of Gaussian fits to the $0^{\circ}$ peak in the two-charged-hadron azimuthal-angle $(\Delta \phi)$ correlation functions [201]. The correlation functions were formed from pairs with trigger hadron in the $p_{T}$ range $2.5<p_{T}<4.0 \mathrm{GeV} / c$ and the associated hadron in the range $1.0<p_{T}<2.5 \mathrm{GeV} / c$. The dashed lines show the $\pm 1 \sigma$ range of the jet widths in $d+\mathrm{Au}$ collisions using the same momentum bins. In the $\mathrm{Au}+\mathrm{Au}$ data, the effect of the elliptic flow has been subtracted in the extraction of the jet width.

tions predict that the fractional energy loss of a propagating parton decreases with increasing parton energy. However, the precise evolution with parton energy depends on the assumptions in the energy loss models and on the treatment of details like kinematic limits and non-leading terms in the radiation spectrum [205,204]. There are many different calculations of medium-induced energy loss currently available based on a variety of assumptions about the thickness of the medium, the energy of the radiating parton, and the coherence in the radiation process itself (see $[208,205,204]$ for recent reviews). The $p_{T}$ dependence of the PHENIX $\pi^{0} R_{A A}$ values has ruled out the possibility of 
a constant (energy independent) $\Delta E / \Delta x[203]$ and the original BDMS energy loss formulation (which the authors argued should not be applied at RHIC energies). In fact, the only detailed energy loss model that predicted the flat $p_{T}$ dependence of $R_{A A}$ over the $p_{T}$ range covered by RHIC data was the GLV prescription $[181,209,210,211,194]$. In the GLV formulation, the fractional energy loss for large jet energies varies approximately as $\log (E) / E$ but the authors observe that below $20 \mathrm{GeV}$ the full numerical calculation of the energy loss produces a nearly constant $\Delta E / E[205]$. However, the same authors argue that the flat $R_{A A}\left(p_{T}\right)$ observed at high $p_{T}$ at $200 \mathrm{GeV}$ also requires an accidental cancellation of several different contributions including the separate $p_{T}$ dependences of the quark and gluon jet contributions, the $p_{T}$ dependence of the Cronin enhancement, and shadowing/EMC effect. A comparison of the GLV results for the $p_{T}$ dependence of the $\pi^{0}$ suppression to the PHENIX data is shown in Fig. 42.

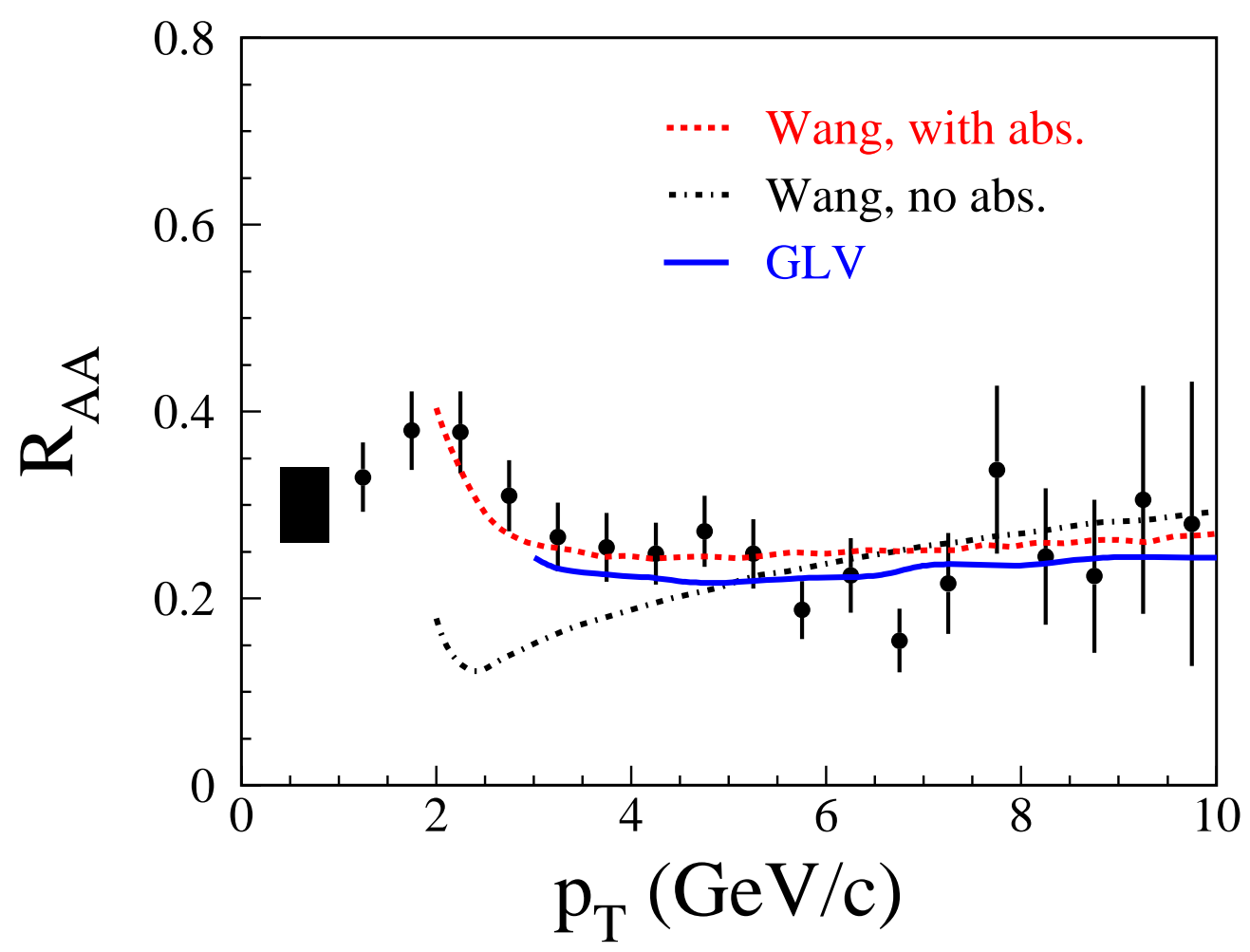

Fig. 42. Comparisons of energy loss calculations $[212,185]$ used to extract estimates for the initial parton number or energy density (see text for details) to the central $200 \mathrm{GeV} \mathrm{Au}+\mathrm{Au} \pi^{0} R_{A A}\left(p_{T}\right)$ measured by PHENIX. The Wang curves compare results with and without energy absorption from the medium.

One of the most critical issues in the energy loss calculation is the treatment of the time evolution of the energy density of the matter through which the radiating parton is propagating. Even if transverse expansion of the created matter is ignored, the longitudinal expansion produces a rapid reduction in the 
energy density as a function of time. Most energy loss calculations assume that the color charge density decreases as a function of proper time as $\rho(\tau)=\rho_{0} \tau_{0} / \tau$ in which case the measured $R_{A A}$ can be used to infer the product $\rho_{0} \tau_{0}$. Here $\tau_{0}$ represents the formation time of the partons from which the medium is composed and $\rho_{0}$ the initial number density of those partons. Since the gluons have the largest cross section for scattering with other partons, the initial color-charge density is interpreted as the gluon density. Making the usual assumption that the produced partons are spread over a longitudinal spatial width $\delta z=\tau_{0} \delta y$, the GLV authors relate the product $\rho_{0} \tau_{0}$ to the initial $d n_{g} / d y$ and obtain $d n_{g} / d y=1000 \pm 200$ from the PHENIX $\pi^{0} R_{A A}$ values [194]. The sensitivity of the GLV calculations to the details of the description of the transverse parton density and the transverse expansion of the matter has been tested by using the results of hydrodynamic calculations of the energy density as a function of position and time [213]. The average energy loss for partons in central $\mathrm{Au}+\mathrm{Au}$ collisions evaluated under dramatically different assumptions was shown to be remarkably insensitive to details of the description of the parton density. The GLV results are also potentially sensitive to a "screening mass" that determines both the transverse momentum distributions of the virtual gluons absorbed from the medium in the bremsstrahlung process and an energy cutoff for the radiated gluons. This mass is related to the local energy density using lattice QCD calculations of the plasma screening mass [194]. However, it was shown by the authors that a factor of two change in the screening mass produces only a $15 \%$ change in the $d n_{g} / d y$ needed to describe the data.

An alternative analysis of parton energy loss [214] starts from explicit calculation of higher-twist matrix elements for $e+A$ collisions that account for coherent rescattering of the struck quark in the nucleus. The contributions of these higher-twist terms can be incorporated into modified jet fragmentation functions, producing an effective energy loss. This calculation can reproduce [188] the HERMES measurements of modified jet fragmentation in nuclear deep-inelastic scattering [215]. By relating the modified fragmentation functions from the higher-twist calculation to energy-loss results obtained from the leading term in an opacity expansion calculation (e.g. GLV) of mediuminduced energy loss the parameters describing the rescattering in the nucleus in $e+A$ collisions can be related to the parameters describing the medium in an explicit energy-loss calculation. By relating the two sets of parameters, the parton density in the hot medium can be related to the parton density in a cold nucleus [188]. Results of this analysis are shown in Fig. 42 for parameters that give an initial energy loss per unit length of $13.8 \pm 3.9 \mathrm{GeV} / \mathrm{fm}$ when the HIJING [79] parameterization of shadowing is used [185] (Note: this result is a factor of two larger that in [188] which was based on analysis of the $130 \mathrm{GeV}$ results). However, an alternative (EKS) [216] shadowing description results in an initial energy loss of $16.1 \pm 3.9 \mathrm{GeV} / \mathrm{fm}$ [185] in the same calculation indicating at least a $25 \%$ systematic error in the energy loss estimates due 
to uncertainties in the description of nuclear shadowing. Nonetheless, these initial-energy-loss values are much larger than the time-averaged energy loss extracted from the calculation, $0.85 \pm 0.24 \mathrm{GeV} / \mathrm{fm}$ for HIJING shadowing [185], due to the assumed $1 / \tau$ decrease in the color-charged density. In fact, the average energy loss per unit path length in central $\mathrm{Au}+\mathrm{Au}$ collisions [188] is comparable to the value for cold nuclear matter extracted from HERMES data [188]. However, the initial energy loss is estimated by Wang to be a factor of $\sim 30$ larger than that in a cold nucleus [185] implying that the initial $\mathrm{Au}+\mathrm{Au}$ parton density is larger by a factor $>30$ than in cold nuclear matter $[217]$.

As shown in Fig. 42 the Wang higher-twist calculation predicts a suppression that varies strongly with $p_{T}$ over the range where the experimental $R_{A A}\left(p_{T}\right)$ values are flat. However, Wang and Wang have argued that absorption of energy from the medium needs to be accounted for in calculating the energy loss of moderate- $p_{T}$ partons [187]. They provide a formula which incorporates both parton energy loss and "feedback" from the medium that can reproduce the shape of the observed high- $p_{T}$ suppression as shown by the lower curve in Fig. 42. This formula, then, provides the energy loss estimate given above. This explanation for the observed $p_{T}$ independence of $R_{A A}$, a crucial feature of the experimental data, is disquieting, however, because it contradicts the explanation provided by the GLV model which provides a consistent estimate of the initial energy density. The feedback of energy from the medium is not included in the GLV calculations and if this contribution is significant, then the agreement of the GLV predictions with the $\pi^{0} R_{A A}\left(p_{T}\right)$ over the entire $p_{T}$ range would have to be considered "accidental". Also, the variation of the suppression in the Wang higher-twist calculation with $p_{T}$ reflects the $\Delta E \propto$ $\log E$ variation of parton energy loss naturally obtained from approximations to the full opacity expansion [205]. As noted above, the GLV approach finds that incorporating non-leading terms in the opacity expansion produces $\Delta E \propto$ $E$. Thus, while the absorption of energy from the medium in the Wang et al. approach may only be significant below $p_{T}=5 \mathrm{GeV} / c$, the differences between the variation of energy loss with parton energy in the two approaches will not be confined to low $p_{T}$.

One source of uncertainty in the interpretation of the high- $p_{T}$ suppression is the role of possible inelastic scattering of hadrons after fragmentation. It was originally argued that final-state inelastic scattering of hadrons could produce all of the observed suppression [218]. The persistence of the jet signal with the correct width in $\mathrm{Au}+\mathrm{Au}$ collisions would be difficult to reconcile with this hypothesis. Indeed, more recent analyses [219] discount the possibility that hadronic re-interaction could account for the observed high- $p_{T}$ suppression and indicate that only $\sim 1 / 3$ of fragmentation hadrons undergo final-state inelastic scattering [219]. Wang has also argued [220] that the complete pattern of high- $p_{T}$ phenomena observed in the RHIC data cannot be explained 
by hadronic rescattering. However, this leaves open the question of whether hadronic re-interactions after jet fragmentation can be partially responsible for the observed high- $p_{T}$ suppression. There are a number of other open issues with the quantitative interpretation of the observed high- $p_{T}$ suppression. The calculations all assume that the jets radiate by scattering off static color charges while the typical initial gluon $p_{T}$ is often assumed to be $\sim 1 \mathrm{GeV}$. Also the radiated gluons are assumed to be massless though a plasmon cutoff equal to the screening mass is applied. The systematic errors introduced by these and other assumptions made in the current energy loss calculations have not yet been evaluated though the gluon screening mass is being included in analyses of heavy-quark energy loss.

\subsection{Empirical Energy Loss Estimate}

The observation that the suppression of high- $p_{T}$ particle production is approximately independent of $p_{T}$ above $4 \mathrm{GeV} / c$ and that the $p+p p_{T}$ spectra are well described by a pure power-law function in the same $p_{T}$ range allows a simple empirical estimate of the energy loss of hard-scattered partons in the medium. The $\pi^{0}$ invariant cross section measured by PHENIX in $p+p$ collisions [60] is found to be well described by a power law

$$
E \frac{d^{3} n}{d p^{3}}=\frac{1}{2 \pi} \frac{d^{2} n}{p_{T} d p_{T} d y}=\frac{A}{p_{T}^{n}}
$$

for $p_{T}>3.0 \mathrm{GeV} / c$ with an exponent $n=8.1 \pm 0.1$. If we assume that none of the hard-scattered partons escape from the medium without losing energy, then the approximately $p_{T}$-independent suppression above $4.5 \mathrm{GeV} / c$ can be interpreted as resulting from an average fractional shift in the momentum of the final-state hadrons due to energy loss of the parent parton. The suppressed spectrum can be evaluated from the unsuppressed $(p+p)$ spectrum by noting that hadrons produced in $\mathrm{Au}+\mathrm{Au}$ collisions at a particular $p_{T}$ value, would have been produced at a larger $p_{T}$ value $p_{T}{ }^{\prime}=p_{T}+S\left(p_{T}\right)$ in $p+p$ collisions. If the energy loss is proportional to $p_{T}$ then we can write $S\left(p_{T}\right)=S_{0} p_{T}$ so $p_{T}{ }^{\prime}=\left(1+S_{0}\right) p_{T}$ Then, the number of particles observed after suppression in a given $\Delta p_{T}$ interval is given by

$$
\frac{d n}{d p_{T}}=\frac{d n}{d p_{T}} \frac{d p_{T}{ }^{\prime}}{d p_{T}}=\frac{A}{\left(1+S_{0}\right)^{(n-2)} p_{T}^{(n-1)}} .
$$

We note that the factor $\frac{d p_{T^{\prime}}}{d p_{T}}$ accounts for the larger relative density of particles per measured $p_{T}$ interval due to the effective compression of the $p_{T}$ scale caused by the induced energy loss; this factor is necessary for the total num- 
ber of particles to be conserved. The nuclear modification factor then can be expressed in terms of $S_{0}$,

$$
R_{A A}\left(p_{T}\right)=\frac{1}{\left(1+S_{0}\right)^{(n-2)}} .
$$

Using this very simple picture, we can estimate the fraction of energy lost by hard-scattered partons in the medium from our measured $R_{A A}$ values. First we obtain $S_{0}$ from Eq. 30

$$
S_{0}=\frac{1}{R_{A A^{1 /(n-2)}}}-1 .
$$

Then we observe that the hadrons that would have been produced in $p+p$ collisions at a momentum $\left(1+S_{0}\right) p_{T}$ were actually produced at $p_{T}$, implying a fractional energy loss

$$
S_{\text {loss }}=1-1 /\left(1+S_{0}\right)=1-R_{A A}^{1 /(n-2)} .
$$

Figure 43 shows the centrality dependence of $S_{\text {loss }}$ obtained from the $p_{T^{-}}$ averaged $R_{A A}$ values shown in Fig. 38. For the most central $\mathrm{Au}+\mathrm{Au}$ collisions at $200 \mathrm{GeV}$ we obtain $S_{\text {loss }}=0.2$, which naively implies that an average $20 \%$ reduction in the energy of partons in the medium will produce the suppression observed in the $\pi^{0}$ spectra above $4.5 \mathrm{GeV} / c$. The extracted $S_{\text {loss }}$ values are well described by an $N_{\text {part }}{ }^{2 / 3}$ dependence using the most central bin to fix the proportionality constant. This result agrees with the GLV prediction for the centrality dependence of the medium-induced energy loss.

It has been shown previously $[221,222]$ that fluctuations in the radiation process can distort an estimate of parton energy loss using the procedure described above. Because of the steeply falling $p_{T}$ spectrum, the partons that lose less energy dominate the yield at a given $p_{T}$ so our determination of $S_{\text {loss }}$ will significantly underestimate the true energy loss. However, it has also been observed that this distortion can largely be compensated by a single multiplicative factor of value $\sim 1.5-2$ [221]. While we cannot use the empirically extracted energy loss to estimate an initial gluon density, we can evaluate the consistency of our results with estimates of $\langle d E / d x\rangle$ in the medium. If we take into account the factor of $1.5-2.0$ renormalization of the $S_{\text {loss }}$, we estimate that $10 \mathrm{GeV}$ partons lose $\sim 3-4 \mathrm{GeV}$ of energy. If the typical path length of these partons is on the order of the nuclear radius then we can infer a $\Delta E / \Delta x \sim 0.5 \mathrm{GeV} / \mathrm{fm}$ which is in good agreement with the estimate from Wang [188]. We can also use the above empirical energy loss approach to evaluate possible systematic errors in the estimate of the initial gluon density. For example, if one third of the observed suppression were a result of finalstate hadronic interactions in the medium, then the suppression due to energy 


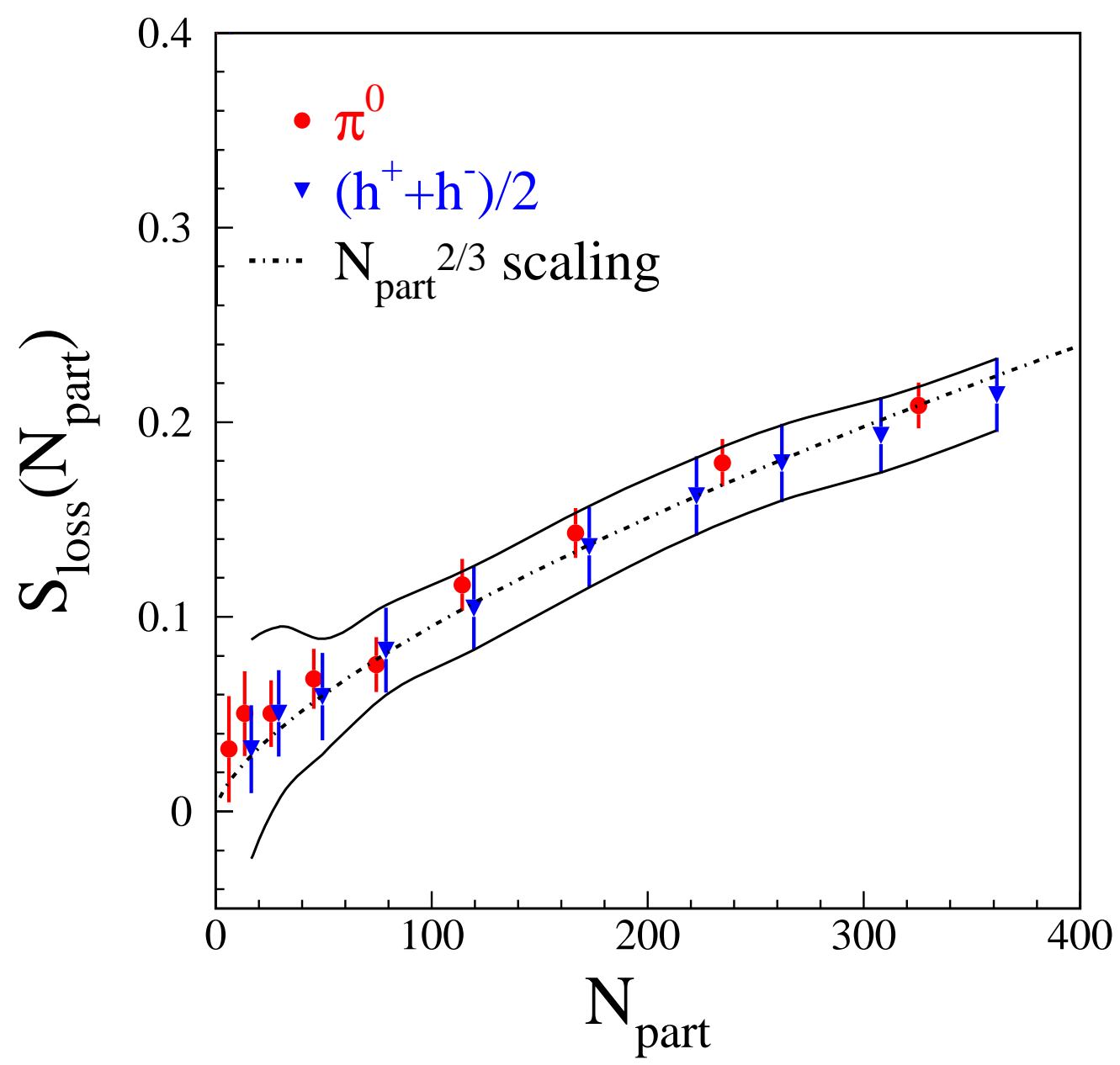

Fig. 43. Calculated energy loss shift factor, $S_{\text {loss }}$ vs. $N_{\text {part }}$ for $\pi^{0}$ and charged hadron production in $200 \mathrm{GeV} \mathrm{Au}+\mathrm{Au}$ collisions. The band around the values indicates systematic errors resulting from uncertainties in $T_{A B}$ and the normalization of the $p+p$ spectrum. The dot-dashed curve shows an $N_{\text {part }}{ }^{2 / 3}$ scaling of $S_{\text {loss }}$ using the most central bin to fix the proportionality constant.

loss would be a factor of 1.5 smaller than that implied by the measured $R_{A A}$ values, assuming that every fragmentation hadron that interacts effectively "disappears" by being shifted to much lower momentum. As a result, $S_{0}$ in central $\mathrm{Au}+\mathrm{Au}$ collisions would be reduced from 0.25 to 0.17 , implying $30 \%$ reduction in the estimated energy loss. If the energy loss is indeed proportional to the initial gluon density then the uncertainty in the effect of the final-state hadronic interactions would introduce a $30 \%$ systematic error in $d n_{g} / d y$. 


\subsection{Conclusions}

The observed suppression of high- $p_{T}$ particle production at RHIC is a unique phenomenon that has not been previously observed in any hadronic or heavy ion collisions at any energy. The suppression provides direct evidence that $\mathrm{Au}+\mathrm{Au}$ collisions at RHIC have produced matter at extreme densities, greater than ten times the energy density of normal nuclear matter and the highest energy densities ever achieved in the laboratory. Medium-induced energy loss, predominantly via gluon bremsstrahlung emission, is the only currently known physical mechanism that can fully explain the magnitude and $p_{T}$ dependence of the observed high- $p_{T}$ suppression. This conclusion is based on evidence provided above that we summarize here:

- Observation of the $x_{T}$ scaling of the high- $p_{T}$ hadron spectra and measurements of two-hadron azimuthal-angle correlations at high $p_{T}$ confirm the dominant role of hard scattering and subsequent jet fragmentation in the production of high- $p_{T}$ hadrons.

- $d+$ Au measurements demonstrate that any initial-state modification of nuclearparton distributions has little effect on the production of hadrons with $p_{T}>2 \mathrm{GeV} / c$ at mid-rapidity.

- This conclusion is further strengthened by preliminary PHENIX measurements showing that the yield of direct photons with $p_{T}>5 \mathrm{GeV} / c$ is consistent with a $T_{A B}$ scaling of a pQCD-calculated $p+p$ direct-photon spectrum.

- Analyses described above indicate that final-state hadronic interactions can only account for a small fraction of the observed high- $p_{T}$ suppression.

Interpreted in the context of in-medium energy loss, the high- $p_{T}$ suppression data rule out the simplest energy loss prescription - a jet energy independent $\Delta E / \Delta x$. The approximately flat $R_{A A}\left(p_{T}\right)$ was predicted by the GLV energy loss model from which the most explicit estimates of the initial gluonnumber density, $d n_{g} / d y=1000 \pm 200$ and a corresponding initial energy density $\varepsilon_{0} \approx 15 \mathrm{GeV} / \mathrm{fm}^{3}$ [194], have been obtained. An alternative estimate from the analysis of Wang et al. [188] yields a path-length-averaged energy loss of $0.5 \mathrm{GeV} / \mathrm{fm}$. Assuming a $1 / \tau$ time evolution of the energy density a much larger initial energy loss of $13-16 \mathrm{GeV} / \mathrm{fm}$ is obtained. That estimate combined with the estimated $0.5 \mathrm{GeV} / \mathrm{fm}$ energy loss of partons in cold nuclear matter yields an initial $\mathrm{Au}+\mathrm{Au}$ gluon density $>30$ times larger than that in nuclei [217]. From this result, Wang concludes that the initial energy density is a factor of $\sim 100$ times larger than that of a nucleus which would correspond to $16 \mathrm{GeV} / \mathrm{fm}^{3}$ [217]. While this conclusion is consistent with the independent estimate from GLV, we note that the two models provide completely different explanations for the nearly $p_{T}$-independent $R_{A A}$ - the most unique feature of the single-particle high- $p_{T}$ suppression - and the differences between the approaches may not be confined to low $p_{T}$. An empirical analysis of the par- 
ton energy loss suggests that the Wang estimate of $>0.5 \mathrm{GeV} / \mathrm{fm}$ for the average parton $\Delta E / \Delta x$ is consistent with the measured $R_{A A}$ values in central $\mathrm{Au}+\mathrm{Au}$ collisions. However, some outstanding issues with current energy loss calculations and the interpretation of high- $p_{T}$ suppression were noted above. Most notably, rescattering of hadrons after parton fragmentation could affect the observed high- $p_{T}$ suppression even if such rescattering cannot explain the pattern of jet quenching observations. Using results from [219] and our empirical energy loss analysis, we estimated that hadronic interactions could modify extracted values for initial parton densities by only $30 \%$. However, we cannot evaluate the potential systematic error in extracted parton densities due to other untested assumptions of the energy loss calculations. Therefore, to be conservative we interpret the extracted initial gluon number and energy densities as order-of-magnitude estimates. Even then, the $15 \mathrm{GeV} / \mathrm{fm}^{3}$ estimated by Gyulassy and Vitev from the central $200 \mathrm{GeV} \mathrm{Au}+\mathrm{Au} \pi^{0} R_{A A}\left(p_{T}\right)$ measurements indicates that the matter produced in central $\mathrm{Au}+\mathrm{Au}$ collision has an energy density $>10$ times normal nuclear matter density.

\section{HADRON PRODUCTION}

Descriptions of heavy ion collisions have provided an understanding of early energy densities, production rates and medium effects of hard partons, and collective flow of matter. However, hadronization - the process by which partons are converted into hadrons - is not well understood. The process of hadronization is particularly important since it includes both the dressing of the quarks from their bare masses, i.e. the breaking of approximate chiral symmetry, and the confinement of quarks into colorless hadrons. One could conclude that a quark-gluon plasma had been formed if one had conclusive evidence of hadronization occurring from a thermal distribution of quarks and gluons.

Hadronization processes have been studied over many years in proton-proton and electron-positron reactions. Hadron formation, by its very nature a nonperturbative process, has often been parameterized from data (e.g. fragmentation functions $D(z)$ ) or phenomenologically described (e.g. string models) [223]. From QCD one expects that hadron production at high transverse momentum is dominated by hard scattering of partons followed by fragmentation into "jets" or "mini-jets" of hadrons. Following the assumptions of collinear factorization, the fragmentation functions should be universal. This universality has proved a powerful tool in comparing $e^{+} e^{-}$annihilation to hadronhadron reactions. One feature of jet fragmentation is that baryons and antibaryons are always suppressed relative to mesons at a given $p_{T}[224,225]$. Phenomenologically this can be thought of as a large penalty for creating a diquark-antidiquark pair for baryon formation vs. a quark-antiquark pair for meson formation. 
In hadron-hadron reactions, hard scattering followed by fragmentation is considered to be the dominant process of hadron production for particles with $p_{T} \geq 2 \mathrm{GeV} / c$ at mid-rapidity. At low transverse momentum, where particles have $p_{T}<2 \mathrm{GeV} / c$, particle interactions are often referred to as "soft". In small momentum transfer reactions the effective wavelength of interactions is longer than the spacing of individual partons in a nucleon or nucleus. Thus coherence effects are expected to result in large violations of factorization and universality of fragmentation functions. Hadron formation mechanisms in this "soft" regime are poorly understood. We are particularly interested in the study of hadron formation in the region of $p_{T} \approx 2-5 \mathrm{GeV} / c$, where production is expected to make the transition from "soft" to "hard" mechanisms.

\subsection{Baryons and Antibaryons}

One of the most striking and unexpected observations in heavy ion reactions at RHIC is the large enhancement of baryons and antibaryons relative to pions at intermediate $p_{T} \approx 2-5 \mathrm{GeV} / c$. As shown in Fig. 44, the (anti)proton to pion ratio is enhanced by almost a factor of three when one compares peripheral reactions to the most central gold-gold reactions $[52]^{7}$. This of course is in sharp contrast to the suppression of pions in this region.

We can investigate this (anti)baryon excess to much higher $p_{T}$ by comparing our inclusive charged spectra (primarily pions, kaons and protons) with our neutral pion measurements [52]. Shown in Fig. 45 is the charged hadron to $\pi^{0}$ ratio as a function of transverse momentum in ten centrality bins. We observe a significant increase of the $\left(h^{+}+h^{-}\right) / \pi^{0}$ ratio above 1.6 in the $p_{T}$ range $1-5 \mathrm{GeV} / c$ that increases as a function of collision centrality. The ratio of $h / \pi=1.6$ is the value measured in $p+p$ reactions [224], and is thought to arise from jet fragmentation. In $\mathrm{Au}+\mathrm{Au}$ central reactions, above $p_{T} \approx 5$ $\mathrm{GeV} / c, h / \pi$ returns to the $p+p$ measured baseline. This implies that the (anti)baryon excess occurs only in the limited $p_{T}$ window $\approx 2-5 \mathrm{GeV} / c$, and then returns to the universal fragmentation function expectation.

As discussed in section 6 , pions in this $p_{T}$ range are suppressed by almost a factor of five relative to binary collision scaling for central $\mathrm{Au}+\mathrm{Au}$ reactions. Thus, one possible interpretation of the large (anti)proton to pion ratio is that somehow the baryons are not suppressed in a manner similar to the pions. Figure 46 shows that in fact (anti)proton production appears to follow binary collision scaling over the transverse momentum range $p_{T}=2-5 \mathrm{GeV} / c[52]$. However, the $h / \pi^{0}$ ratios shown in Fig. 45 imply that above $p_{T}>5 \mathrm{GeV} / c$, the (anti)protons must be as suppressed as the pions.

7 All PHENIX (anti)proton spectra shown in this section are corrected for feed down from heavier resonances. 


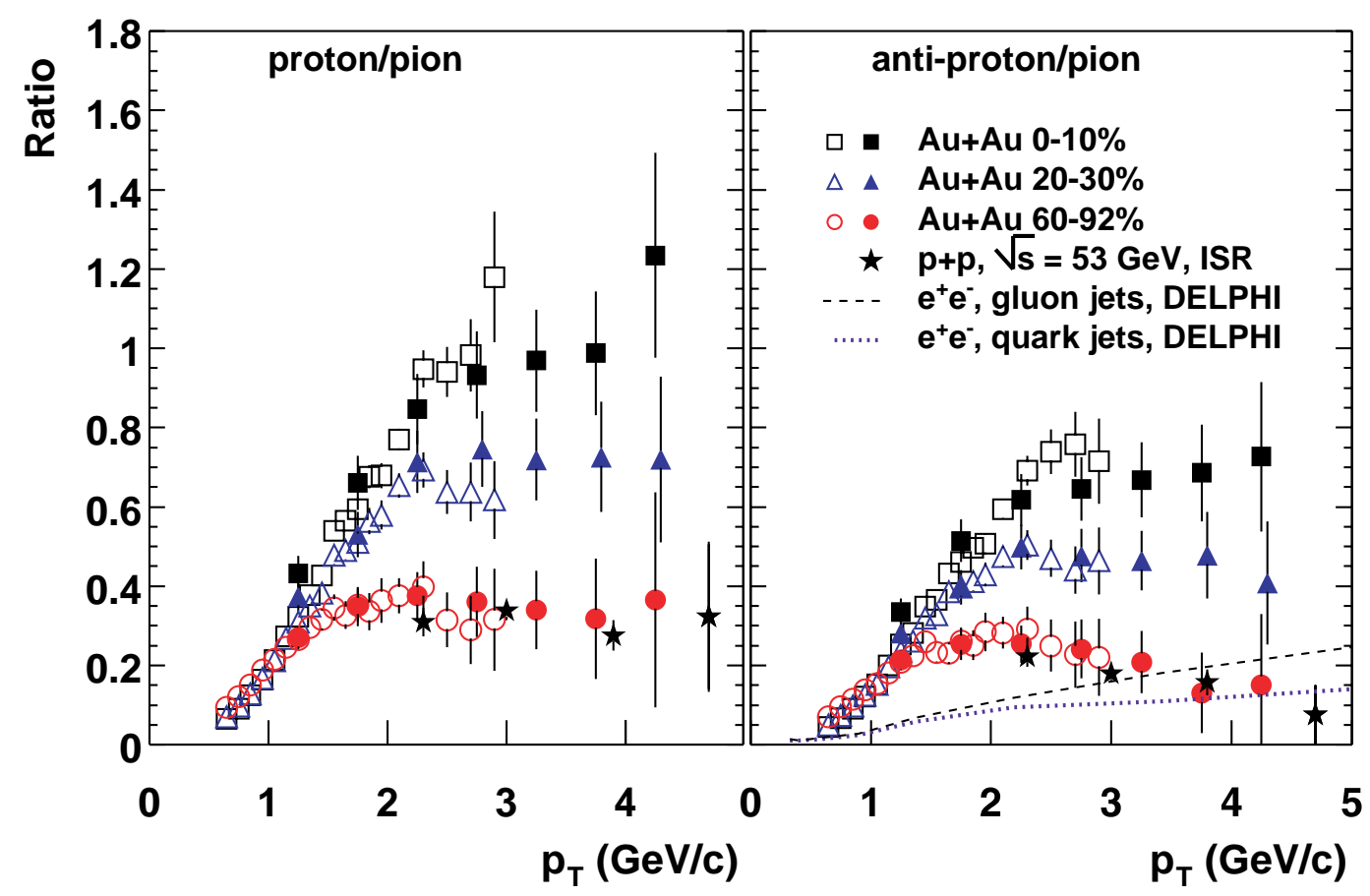

Fig. 44. $p / \pi$ (left) and $\bar{p} / \pi$ (right) ratios for central (0-10\%), mid-central (20-30\%) and peripheral (60-92\%) Au+Au collisions at $\sqrt{s_{N N}}=200 \mathrm{GeV}[52]$. Open (filled) points are for $\pi^{+/-}\left(\pi^{0}\right)$, respectively. Data from $\sqrt{s}=53 \mathrm{GeV} p+p$ collisions [224] are shown with stars. The dashed and dotted lines are $(\bar{p}+p) /\left(\pi^{+}+\pi^{-}\right)$ratio in gluon and in quark jets [225].

Characteristics of the intermediate $p_{T}$ (anti)protons are:

- A large enhancement of the $p / \pi$ and $\bar{p} / \pi$ ratios in central $\mathrm{Au}+\mathrm{Au}$ collisions.

- A ratio in peripheral collisions which is in agreement with that from $p+p$ collisions.

- A smooth increase from peripheral to central $\mathrm{Au}+\mathrm{Au}$ collisions.

- A similar effect for protons and antiprotons.

- Approximate scaling of (anti)proton production at $p_{T} \approx 2-4 \mathrm{GeV} / c$ with the number of binary nucleon-nucleon collisions.

- Suppression relative to binary collision scaling similar for (anti)protons and pions for $p_{T}>5 \mathrm{GeV} / c$.

Large proton to pion ratios have also been observed in heavy ion collisions at lower energies. Figure 47 shows $p_{T}$ distributions of protons, antiprotons, and pions in central $\mathrm{Pb}+\mathrm{Pb}$ collisions at the SPS and in central $\mathrm{Au}+\mathrm{Au}$ collisions at the AGS. The $p / \pi$ ratio in central $\mathrm{Pb}+\mathrm{Pb}$ collisions at the SPS is greater than unity for $p_{T} \geq 1.3 \mathrm{GeV} / c$. At the AGS, the proton spectrum crosses pion spectra at $p_{T} \sim 0.5 \mathrm{GeV} / c$, and the $p / \pi$ ratio is about 20 at $p_{T}=1.6$ $\mathrm{GeV} / c$. The $p / \pi$ ratios in the low-energy heavy-ion collisions are also enhanced compared with $p+p$ collisions at the same energy. 


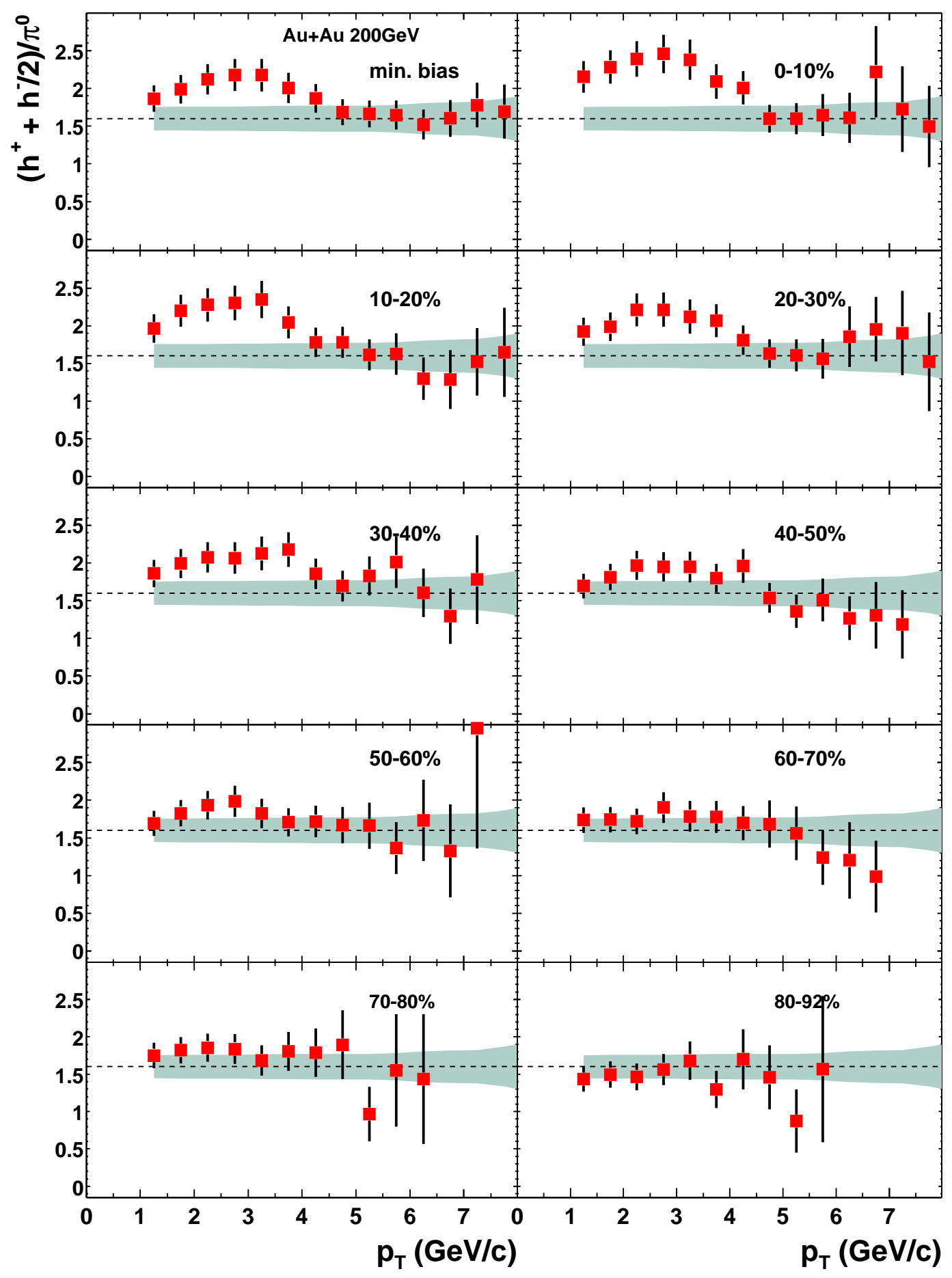

Fig. 45. Charged hadron to $\pi^{0}$ ratio for different centrality classes for $\mathrm{Au}+\mathrm{Au}$ collisions at $\sqrt{s_{N N}}=200 \mathrm{GeV}[53]$. Error bars represent the quadratic sum of statistical and point to point systematic errors. The shaded band shows the normalization error common to all centrality classes. The line at 1.6 is the $h / \pi$ ratio measured in $p+p$ collisions at $\sqrt{s}=53 \mathrm{GeV}[224]$ and e+e- collisions [225]. 


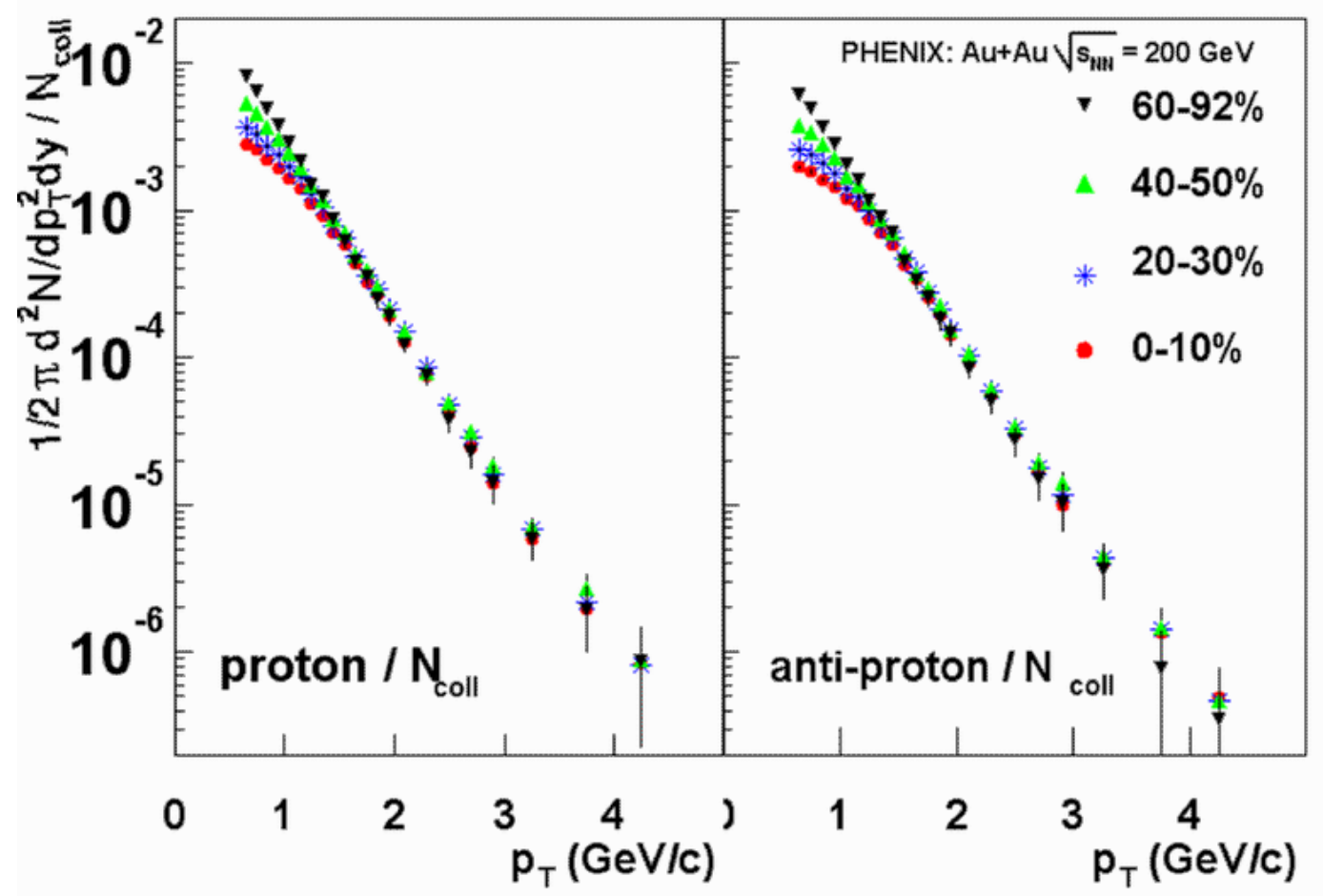

Fig. 46. $p$ and $\bar{p}$ invariant yields scaled by $N_{\text {coll }}$ in $\mathrm{Au}+\mathrm{Au}$ collision at $\sqrt{s_{N N}}=200$ $\mathrm{GeV}[52]$. Error bars are statistical. Systematic errors on $N_{\text {coll }}$ range from $\approx 10 \%$ for central to $\approx 28 \%$ for $60-92 \%$ centrality. Multiplicity dependent normalization errors are $\approx 3 \%$.

Most of the protons in these lower-energy heavy-ion collisions are not produced in the collision. Rather they are protons from the beam or target nucleus $(\mathrm{Pb}$ or $\mathrm{Au}$ ) that are transported to large $p_{T}$ at mid-rapidity. As discussed in section 3 , a strong radial flow with velocity $\beta_{T} \sim 0.5$ is produced in heavy ion collisions at AGS and SPS energies. The large $p / \pi$ ratio can be interpreted as a result of this radial flow. Since the proton is heavier, a fixed velocity boost results is a larger momentum boost than for pions, and thus enhances $p / \pi$ ratio at higher $p_{T}$. In contrast, at RHIC energies, most of protons are produced particles [40]. The anomalously large antibaryon-to-meson ratio $\bar{p} / \pi \sim 1$ at high $p_{T} \geq 2$ $\mathrm{GeV} / c$ is a unique result from $\mathrm{RHIC}$. Such a large $\bar{p} / \pi$ ratio has not been observed in any other collision system. Figure 47 shows that $\bar{p} / \pi$ is less than $\sim 0.1$ at the SPS, and it is less than 1/100 at the AGS. It should also be noted that the measurements from the AGS/SPS are limited to lower $p_{T}\left(p_{T}<2\right.$ $\mathrm{GeV} / c$ ), where soft physics is still dominant, while at RHIC we observe a large $p(\bar{p}) / \pi$ ratio in $p_{T} \approx 2-5 \mathrm{GeV} / c$ where hard processes are expected to be the dominant mechanism of particle production. 

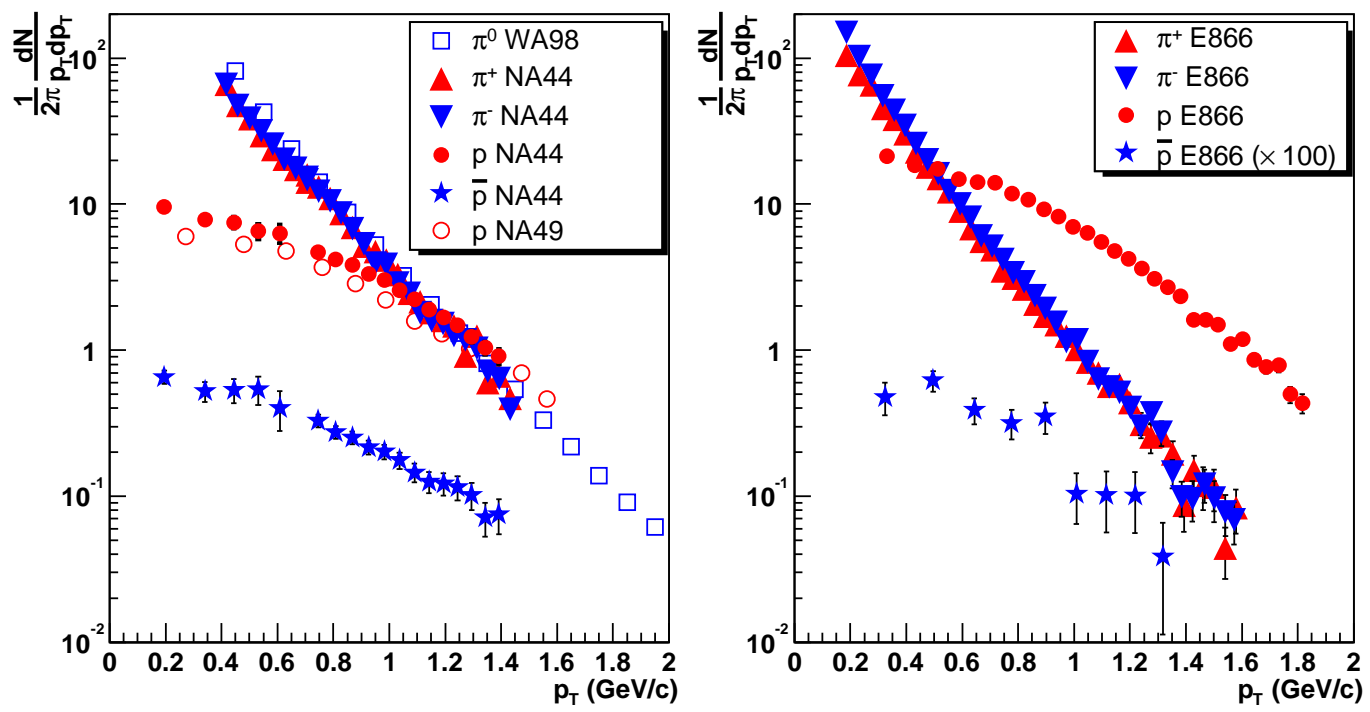

Fig. 47. Invariant yields of $p, \bar{p}$, and $\pi$ as function of $p_{T}$ in central $\mathrm{Pb}+\mathrm{Pb}$ collisions at the SPS $\left(\sqrt{s_{N N}}=17 \mathrm{GeV}\right)$ (left panel) and in central $\mathrm{Au}+\mathrm{Au}$ collisions at the AGS $\left(\sqrt{s_{N N}}=5 \mathrm{GeV}\right)$ (right panel). The $\bar{p}$ spectrum from the AGS is scaled up by a factor 100 . All data are at mid-rapidity $\left(y-y_{c m} \approx 0\right)$ and are from W98 [163], NA44 [226], NA49 [227], and E866 [228,229].

\subsection{The $\phi$ Meson}

We have extended our identified hadron studies to include the $\phi$ vector meson as measured in the $K^{+} K^{-}$decay channel. The $\phi$ is a meson, and is in that sense similar to the pion with a valence quark and antiquark, and yet its mass is comparable to that of the proton.

Figure 48 shows $R_{C P}$, the ratio of production in central to peripheral $\mathrm{Au}+\mathrm{Au}$ collisions scaled by binary collisions, for protons, pions and $\phi$ mesons detected via its $K K$ decay channel [69] in $\mathrm{Au}+\mathrm{Au}$ collisions at $\sqrt{s_{N N}}=200 \mathrm{GeV}$. A large suppression of pions at $p_{T}>2 \mathrm{GeV} / c$ is observed (as detailed in Section 6 ), and a lack of suppression for the protons and antiprotons as expected from Fig. 46. The $\phi$ follows the suppression pattern of the pions within errors, indicating that the surprising behavior of the protons is not followed by the $\phi$. Figure 49 shows a comparison between the $p_{T}$ spectral shape for protons and the $\phi$ in central and peripheral $\mathrm{Au}+\mathrm{Au}$ reactions. The two spectra agree with each other within errors for the most central events. Thus, although the yields are evolving differently with collision centrality, giving rise to the deviation from unity of $R_{C P}$, the $p_{T}$ distributions appear quite similar. 


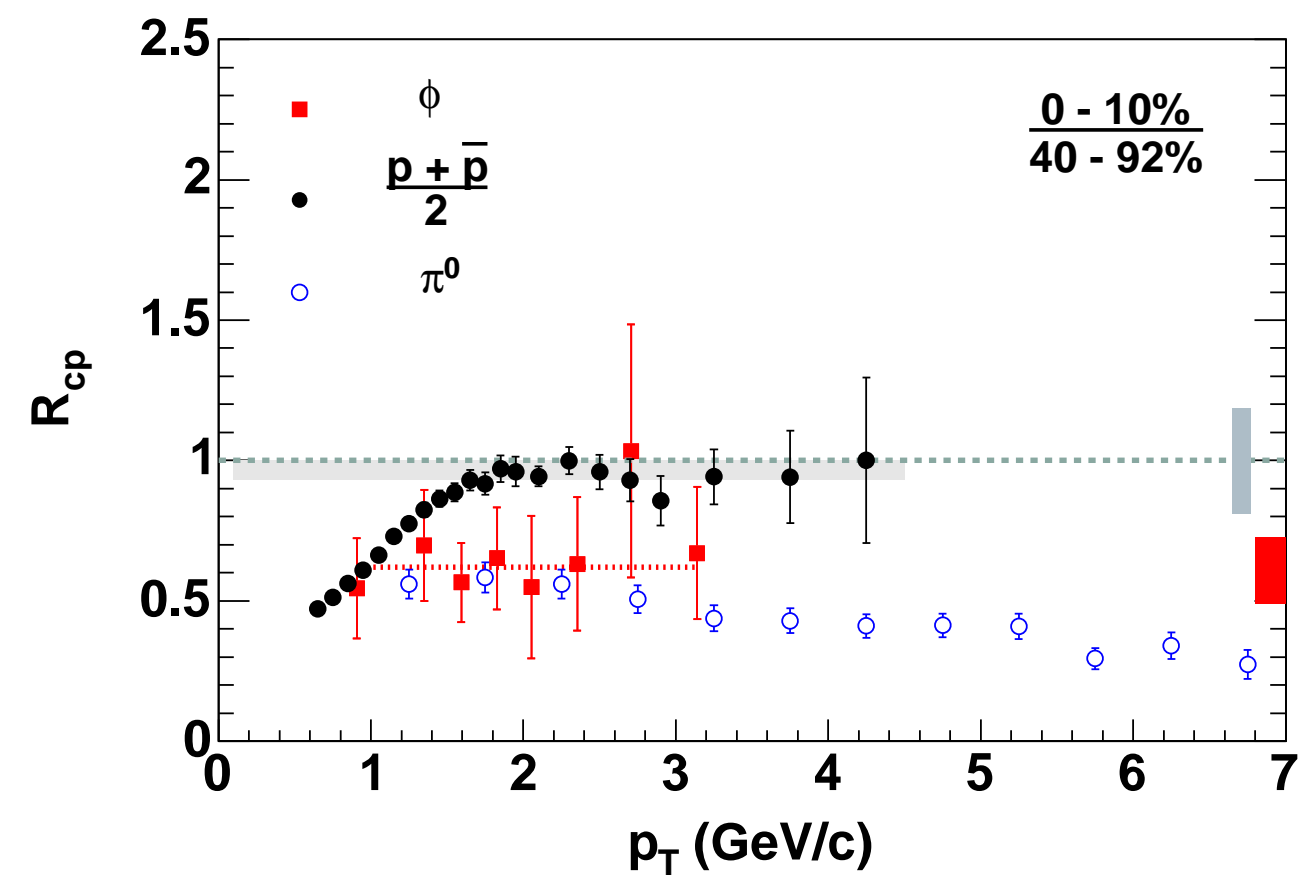

Fig. 48. The $R_{C P}$ of the $\phi$ as measured in the $K K$ channel, compared to the protons and pions for $\mathrm{Au}+\mathrm{Au}$ collisions at $\sqrt{s_{N N}}=200 \mathrm{GeV}[69]$.

\subsection{Jet Correlations}

A crucial test of the origin for the enhanced (anti)proton to pion ratio is to see if baryons in this intermediate $p_{T}$ regime exhibit correlations characteristic of the structure of jets from hard-scattered partons. Particles which exhibit these correlations are termed "jet-like". Figure 50 shows the associated partner particle yield within the relative angular range $0.0<\phi<0.94$ radians on the same side as trigger baryons and mesons [71]. Correlated pairs are then formed between the trigger particle and other particles within the above mentioned angular range. Mixed events are used to determine the combinatorial (i.e. non-jet-like) background distribution, which is subtracted after modulation according to the measured $v_{2}$.

The partner yield increases for both trigger baryons and mesons by almost a factor of two from deuteron-gold to peripheral and mid-central $\mathrm{Au}+\mathrm{Au}$ reactions. We then observe a decrease in the jet-like correlations for baryons relative to mesons for the most central collisions. It is notable that this observation is of limited significance within our current statistical and systematic errors. Over a broad range of centrality 10-60\% the partner yield is the same for protons and pions within errors. This is notable since the (anti)proton to pion ratio has already increased by a factor of two for mid-central $\mathrm{Au}+\mathrm{Au}$ relative to proton-proton reactions, with the implication that the increase in 


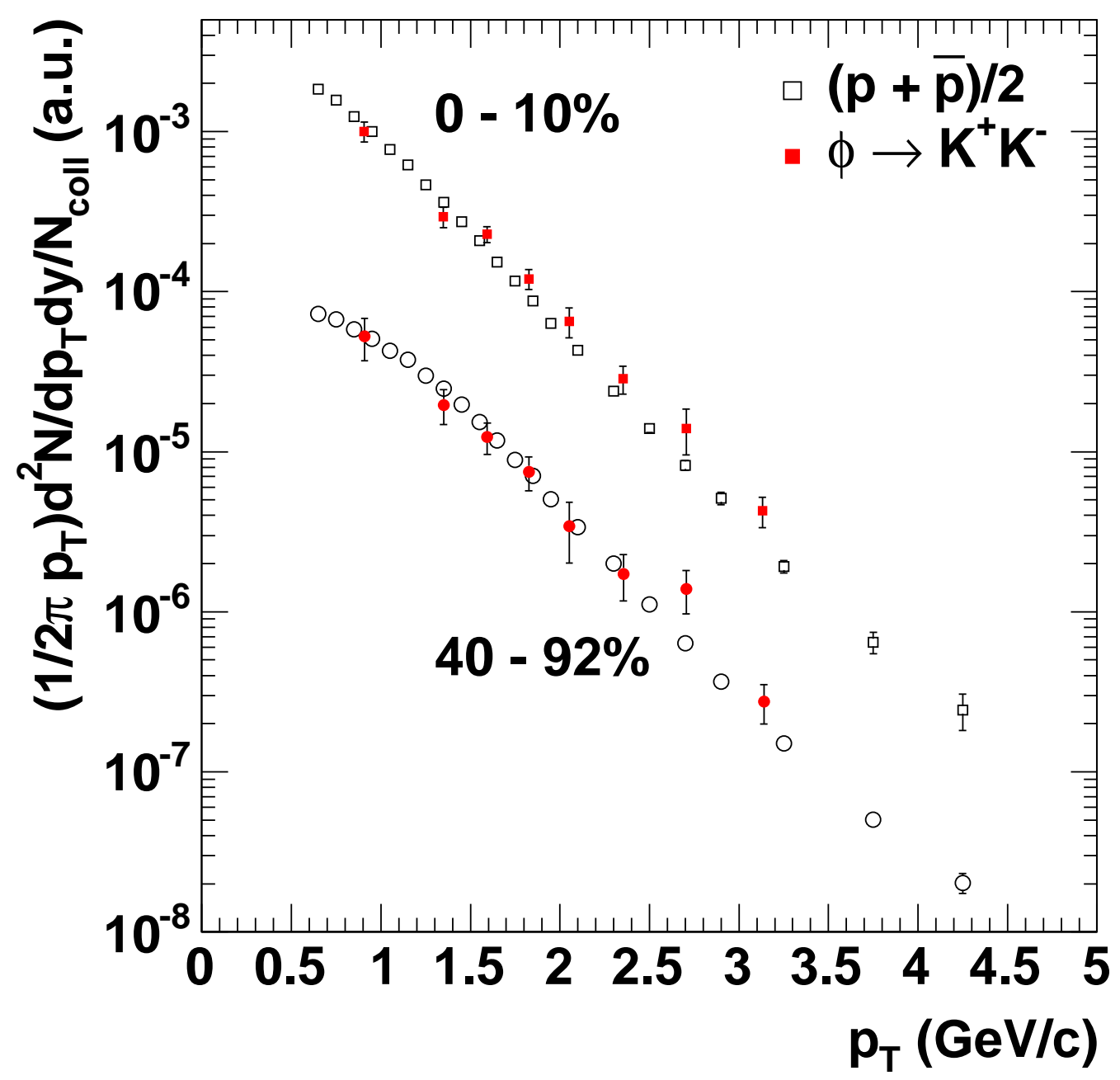

Fig. 49. $(p+\bar{p})$ and $\phi$ invariant yield as a function of transverse momentum for central 0-10\% and peripheral 40-92\% $\mathrm{Au}+\mathrm{Au}$ reactions at $\sqrt{s_{N N}}=200 \mathrm{GeV}$. The two distributions for each centrality class are given an arbitrary relative normalization to allow for comparison of the $p_{T}$ dependent shapes. Data are from [69].

the $p / \pi$ ratio is inclusive of the particles with jet-like correlations.

The dashed line in Figure 50 shows the expected centrality dependence of partners per baryon if all the "extra" baryons which increase the $p / \pi$ over that in $\mathrm{p}+\mathrm{p}$ collisions were to arise solely from soft processes. Baryons from thermal quark recombination should have no jet-like partner hadrons and would dilute the per-trigger conditional yield. Because this simple estimate does not allow for meson production by recombination, which must also occur along with baryon production, it represents an upper limit to the centrality dependence of jet partner yield from thermal recombination. The data clearly disagree with both the centrality dependence and also the absolute yields of 
this estimation, indicating that the baryon excess has the same jet-like origin as the mesons, except perhaps in the highest centrality bin.

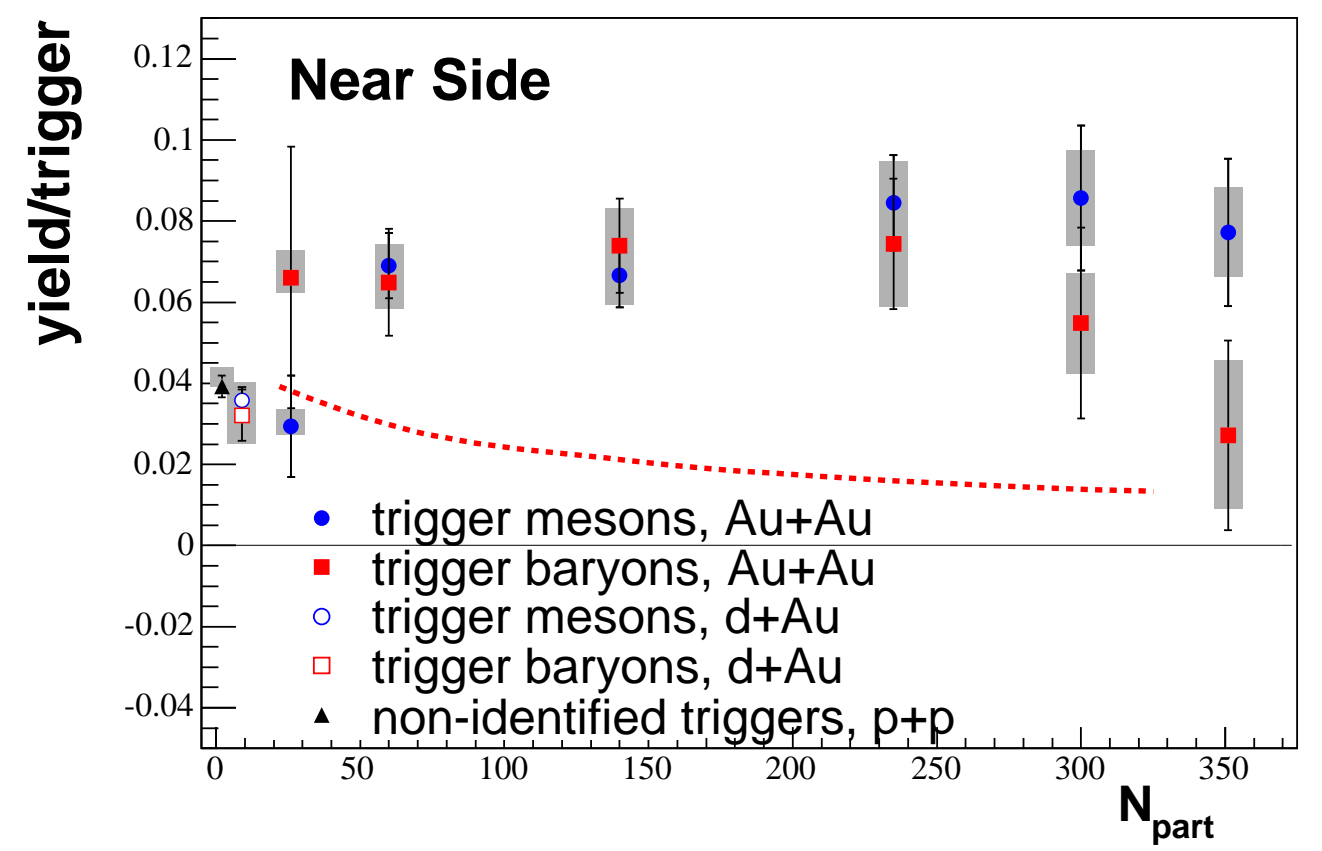

Fig. 50. Centrality dependence of associated charged hadron yield $\left(1.7<p_{T}<2.5\right.$ $\mathrm{GeV} / c$ ) above combinatorial background for trigger baryons and trigger mesons in the $\mathrm{p}_{T}$ range $2.5-4.0 \mathrm{GeV} / \mathrm{c}$ in a $54^{\circ}$ cone around the trigger particle in $\mathrm{Au}+\mathrm{Au}$ collisions at $\sqrt{s_{N N}}=200 \mathrm{GeV}[71]$. The error bars are statistical errors and the gray boxes are systematic errors. The dashed line represents an upper limit of the centrality dependence of the near-side partner yield from thermal recombination (see text).

The characteristics of the jet-like particles are compared to inclusive hadrons in Fig. 51, which shows the centrality dependence of the $p_{T}$ distributions of jet-like partners and inclusive hadrons. One can see that, within the statistics available, the slopes of the associated particle spectra in $p+p, d+\mathrm{Au}$, peripheral and mid-central $\mathrm{Au}+\mathrm{Au}$ collisions are very similar for both trigger mesons and trigger baryons. The partner spectra are harder than the inclusive hadron spectra, as expected from jet fragmentation. In the most central collisions, the number of particles associated with trigger baryons is very small, resulting in large statistical error bars. However, the inverse slopes of the jet-like partners and inclusive hadron distributions agree better in central collisions than in peripheral collisions.

We can then make the following general observations:

- Trigger (anti)protons and mesons have comparable near-side associatedparticle yields over a broad range in centrality, indicating a significant jetlike component for both. 
- There is an indication that the proton partner yield tends to diminish for the most central collisions, unlike for leading mesons.

- Within the limited statistics available for the measurement, the inverse slopes of the associated particles are similar for both mesons and baryons. These are harder than for the inclusive spectra.

- Trigger particles in Au+Au collisions appear to have more associated particles than in $d+\mathrm{Au}$ collisions. This is true for all centralities aside from the most peripheral, and except for leading baryons in central collisions.

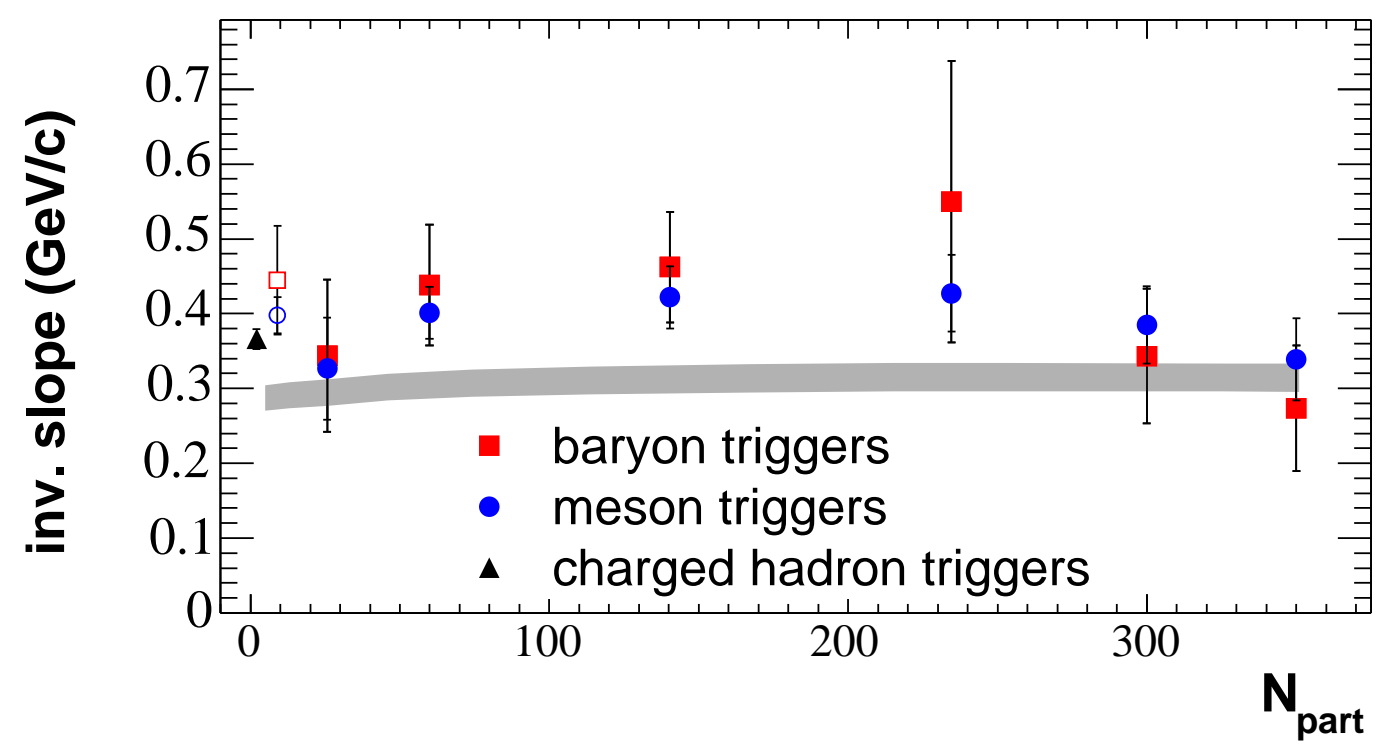

Fig. 51. The inverse slopes for the momentum distributions of the associated particles shown in Fig. 50. The gray band is the inverse slope of the momentum distribution of the inclusive hadrons from $\mathrm{Au}+\mathrm{Au}$ collisions at $\sqrt{s_{N N}}=200 \mathrm{GeV}[71]$.

\subsection{Soft Physics}

In hadron-hadron reactions, hard scattering followed by fragmentation is considered to be the dominant process of hadron production with $p_{T} \geq 2 \mathrm{GeV} / c$ at mid-rapidity. However, as detailed in section 3, there is strong evidence for explosive collective motion of particles in the medium. If the mean free path for particles in the medium is small, then all particles must move with a common local velocity as described by hydrodynamics. Therefore, heavier particles receive a larger momentum boost than lighter particles. This effective shifting of particles to higher $\mathrm{p}_{T}$ results in a "shoulder-arm" shape for the (anti)proton $p_{T}$ spectra, visible in Fig. 49. 


\subsubsection{Hydrodynamics}

Is it possible that this soft hadron production extends to higher $p_{T}$ for baryons than mesons? Hydrodynamic boosting of "soft" physics for heavier particles into the $p_{T}>2 \mathrm{GeV} / c$ offers a natural explanation for the enhanced $p / \pi$ and $\bar{p} / \pi$ ratios $[106]$.

As seen in Section 3, some hydrodynamical models can describe both the proton and the pion spectra. Consequently, the $p / \pi$ ratio is also reproduced (Fig. 52 ). It is clear that the description of the $p / \pi$ ratio is not unique and different calculations yield quite different results. Above some $p_{T}$, hydrodynamics should fail to describe the data and fragmentation should dominate. Pure hydrodynamics predicts that this ratio would continue to increase essentially up to $p_{T} \rightarrow \infty$. However, these particles cannot have a zero mean free path in the medium. Any finite mean free path and a finite volume will limit the number of $p_{T}$ "kicks" a particle can receive. For this reason many of the hydrodynamic calculations are not extended into the $p_{T}$ region $2-5 \mathrm{GeV} / c$ in which we are interested.

Hydrodynamic calculations do not specify the quanta that flow; rather they assume an equation of state. When applied at RHIC, most calculations start with a quark-gluon-plasma equation of state and transition to a resonance gas. The mapping of the fluid onto hadrons is somewhat ad hoc, and often uses the Cooper-Frye freezeout [126], giving the typical hierarchies of momenta one sees where heavier particles receive a larger boost.

As mentioned previously, this generic feature of a transverse velocity boost yielding an increase in the baryon to meson ratio relative to proton-proton reactions is not unique to RHIC as shown in Fig. 47. However, a major difference between lower-energy results and those at $\sqrt{s_{N N}}=200 \mathrm{GeV}$ is that at these highest energies there is a significant hard-process contribution. If the source of the excess baryons is the transport of soft baryons to the intermediate $p_{T}$ range, then it is purely coincidental that the baryons scale with binary collisions. More importantly, we should expect a significant decrease in the jet-like partner yield for baryons relative to mesons. Although there may be a hint of this for the most central reactions, one expects this decrease to follow the centrality dependence of the increase in $p / \pi$ ratio. Thus, this effect should already reduce the partner yield by a factor of two in mid-central $\mathrm{Au}+\mathrm{Au}$ reactions. This is ruled out by the data.

\subsubsection{Recombination Models}

The quark recombination or coalescence model is a different physics framework in which baryons receive a larger $p_{T}$ boost than mesons. These models were frequently invoked in the 1970's [230,231] in an attempt to describe the 


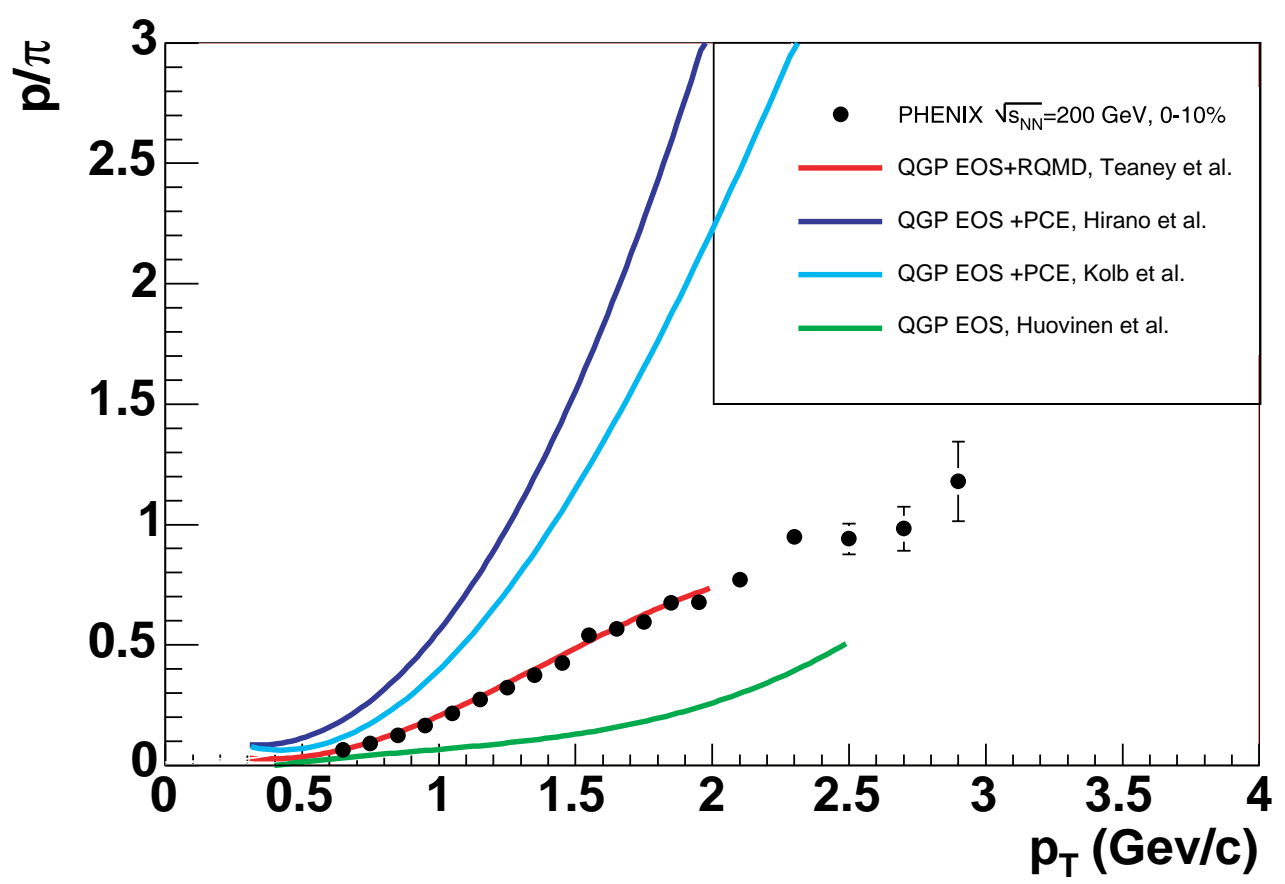

Fig. 52. $p / \pi$ ratios for central $(0-10 \%) \mathrm{Au}+\mathrm{Au}$ collisions at $\sqrt{s_{N N}}=200 \mathrm{GeV}[52]$ compared to hydrodynamic models [106,107,117,118,119].

rapidity distribution of various hadronic species in hadron - hadron reactions. More recently, these models have been applied to describe the forward charm hadron production in hadron - nucleus reactions at Fermilab [232]. In this case they calculate a significant probability for $D$ meson formation from a hard-scattering-created charm quark with a light valence quark in the projectile. The quark coalescence mechanisms have some similarities to light nuclei coalescence. However, wave functions are relatively well determined for light nuclei, whereas the hadron wave functions are neither easily described by partons nor directly calculable from QCD.

Recently, quark recombination has been successfully applied to describe a number of features of heavy ion collisions [233,234] (Duke model). In this picture, quarks in a densely populated phase space combine to form the finalstate hadrons. This model uses the simplifying assumption that the mass is small relative to the momentum giving a prediction largely independent of the final hadron wave function ${ }^{8}$. The coalescing parton distribution was assumed to be exponential, i.e. thermal, and recombination applied for hadrons where $m^{2} / p_{T}^{2}<<1$. At very high $p_{T}$ particles are assumed to arise from fragmentation of hard partons with a standard power law distribution; the relative normalization of the thermal source with respect to this process is an important external parameter to the model. A crucial component of recombination

8 The recombination model prediction of these models is independent of the final hadron wave function with an accuracy of about $20 \%$ for protons and $10 \%$ for pions 
models is the assumption that the partons which recombine carry a mass which is essentially equal to the mass of the dressed constituent quarks ${ }^{9}$. If all observables of intermediate $p_{T}$ hadrons can be explained by recombination of only thermal quarks, this would essentially prove the existence of a quark-gluon plasma in the early stage of the collisions.

Three essential features are predicted by recombination models. First, baryons at moderate $p_{T}$ are greatly enhanced relative to mesons as their transverse momentum is the sum of 3 quarks rather than 2. Recombination dominates over parton fragmentation in this region, because, for an exponential spectrum recombination is a more efficient means of producing particles at a particular $p_{T}$. This enhancement should return to its fragmentation values at higher $p_{T}$. In the intermediate range, all mesons should behave in a similar manner regardless of mass, as should all baryons. Secondly, recombination predicts that the collective flow of the final-state hadrons should follow the collective flow of their constituent quarks. Finally, recombination causes thermal features to extend to higher transverse momentum, $p_{T}>>T_{C}$ than one might naively expect since the underlying thermal spectrum of the constituents gets a multiplication factor of essentially 3 for baryons and 2 for mesons. A last general feature which is true for the simplest of the models, but may not necessarily be true for more complex models, is that at intermediate $p_{T}$, recombination is the dominant mechanism for the production of hadrons - particularly of baryons.

Other recombination calculations have relaxed the assumptions previously described, at the cost of much more dependence on the particular form of the hadronic wave function used. One such calculation [235,236] (Oregon model) uses a description of hadronization which assumes that all hadrons - including those from fragmentation - arise from recombination. Hard partons are allowed to fragment into a shower of partons, which can in turn recombineboth with other partons in the shower and partons in the thermal background. Another model [237] (TAMU model) uses a Monte-Carlo method to model the production of hadrons allowing recombination of hard partons with thermal partons, and includes particle decays, such as $\rho \rightarrow 2 \pi$ which produces low- $p_{T}$ pions.

Figure 53 shows several recombination model calculations compared to the $p / \pi$ ratio from PHENIX. The general features at $p_{T}>3 \mathrm{GeV} / c$ are reasonably reproduced - that is the protons show a strong enhancement at moderate $p_{T}$ which disappears at $p_{T}>5 \mathrm{GeV} / c$ consistent with the measured $\mathrm{h} / \pi$ ratio shown in Fig. 45. The more complicated models do a better job, as one might

9 The actual source of this mass is under discussion. It may be that the chiral phase transition is slightly above the deconfinement transition. In this case, the mass would be from the dressing of the quarks. Another possibility is that the mass is a thermal mass which happens to be similar to the constituent quark mass. 


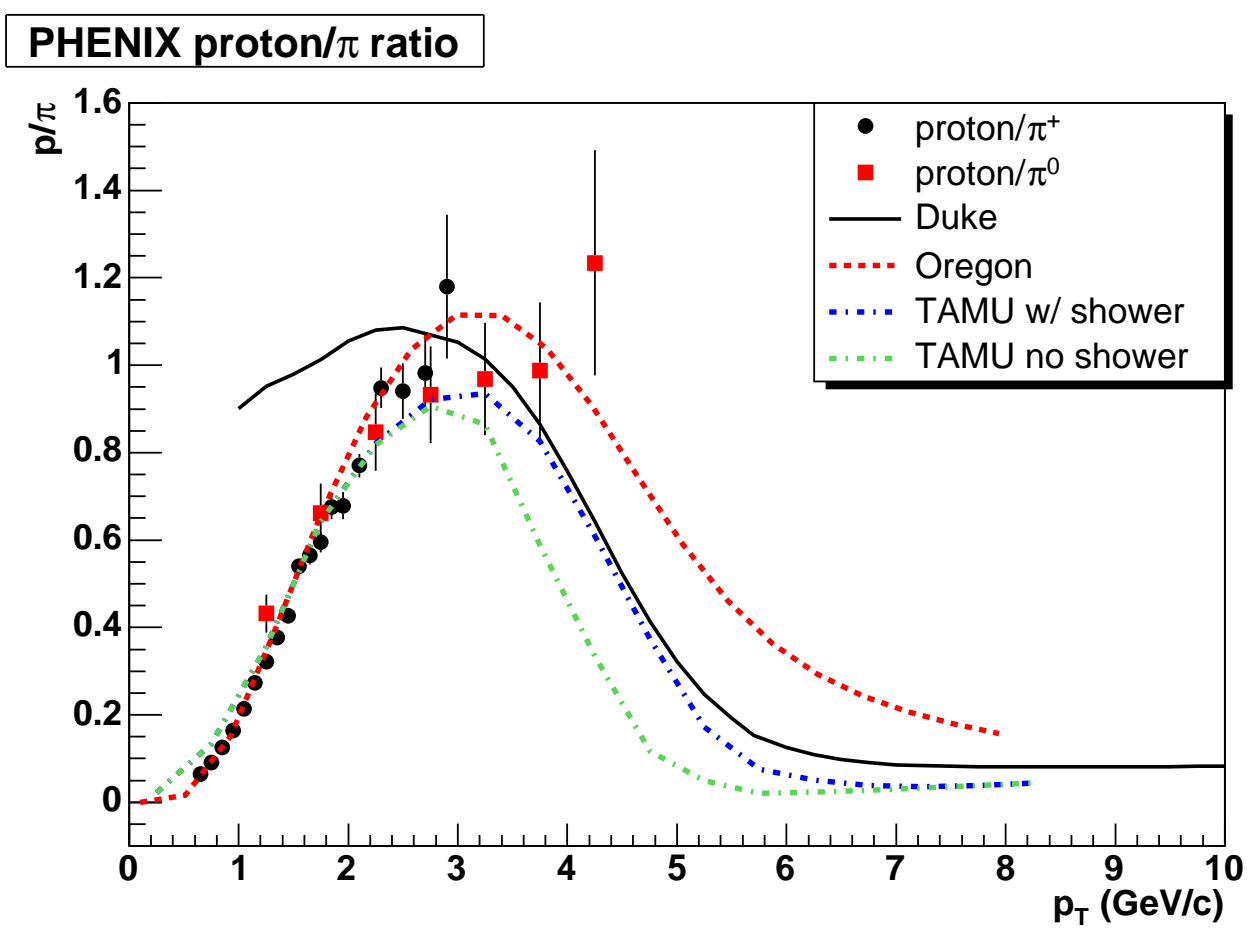

Fig. 53. The proton to pion ratio measured by PHENIX for $\mathrm{Au}+\mathrm{Au}$ collisions at $\sqrt{s_{N N}}=200 \mathrm{GeV}[52]$. Several comparisons to recombination models as mentioned in the text are shown.

expect in the $p_{T}<3 \mathrm{GeV} / c$ region, where the assumptions made by the Duke model begin to break down. Since the recombination model's essential ingredient is the number of constituent quarks in a hadron, the similarity of $R_{C P}$ for the $\phi$ and pions is nicely explained.

Figure 54 shows the fraction of hadrons arising from recombination of only thermal quarks, as a function of $p_{T}$. For $p_{T}$ between 2.5 and $4 \mathrm{GeV} / c$ the fraction of protons from recombination is greater than $90 \%$ for all impact parameters, and is essentially $100 \%$ for the most central collisions. For pions the value is between 40 and $80 \%$, depending on the centrality. This is contradicted by the data in Fig. 50 which clearly shows jet-like correlations for both pions and protons in mid-central collisions. It should be noted that the yield of particles associated with baryons in very central collisions appears to decrease, indicating a possible condition where the simple picture of recombination of purely thermal quarks may apply.

One can examine the general prediction for the elliptic flow of identified particles by rescaling both the $v_{2}$ and the transverse momentum by the number of constituent quarks as shown in Fig. 55. This scaling was first suggested by Voloshin [238]. Above $p_{T} / n$ of $1 \mathrm{GeV} / c$ (corresponding to $3 \mathrm{GeV} / c$ in the proton transverse momentum) all particles essentially plateau at a value of about 0.35 presumably reflecting the elliptic flow of the underlying partons. 


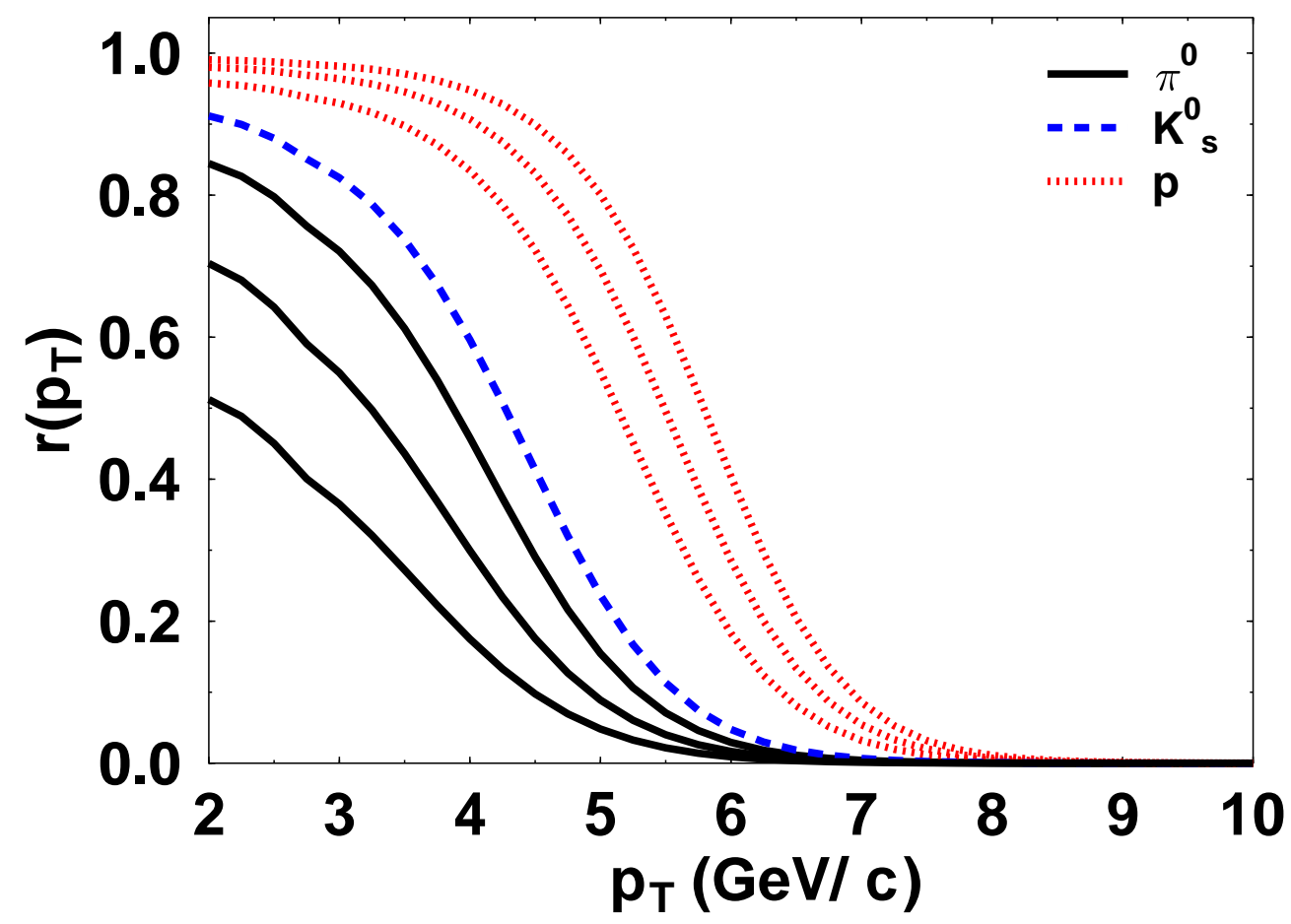

Fig. 54. The ratio $r\left(P_{T}\right)=R /(R+F)$ of recombined hadrons to the sum of recombination $(R)$ and fragmentation $(F)$ for pions (solid), $K^{0} \mathrm{~s}$ (dashed) and $p$ (dotted lines) [234] in $\mathrm{Au}+\mathrm{Au}$ collisions at $\sqrt{s_{N N}}=200 \mathrm{GeV}$. For protons and pions different impact parameters $b=0,7.5$ and $12 \mathrm{fm}$ (from top to bottom) are shown. $K^{0} \mathrm{~s}$ is for $b=0 \mathrm{fm}$ only.

Interestingly, even at lower values of the transverse momentum, all particles also fall on the same curve aside from pions.

It is clear from the jet correlations observed that the majority of moderate $p_{T}$ baryons in peripheral and mid-central collisions cannot arise from a purely thermal source, as that would dilute the per-trigger partner yield. The jet structure and collision scaling indicate that at least some of the baryon excess is jet-like in origin. The relatively short formation time for baryons of such momenta suggests that allowing recombination of fragmentation partons with those from the medium may solve the problem and better reproduce the data. Both the Oregon and TAMU models have mechanisms to do this. However, such modification of the jet fragmentation function must also modify the elliptic flow, and could break the quark scaling needed to reproduce the observed $v_{2}$ trends. Hence, the jet structure of hadrons at 2-5 GeV/c $p_{T}$ presents a challenge to models of the hadron formation.

Figure 56 shows a comparison of the elliptic flow calculated by the TAMU model [241] with PHENIX data from $\mathrm{Au}+\mathrm{Au}$ collisions at $\sqrt{s_{N N}}=200 \mathrm{GeV}$. The model includes the recombination of hard and soft partons, as well as the 


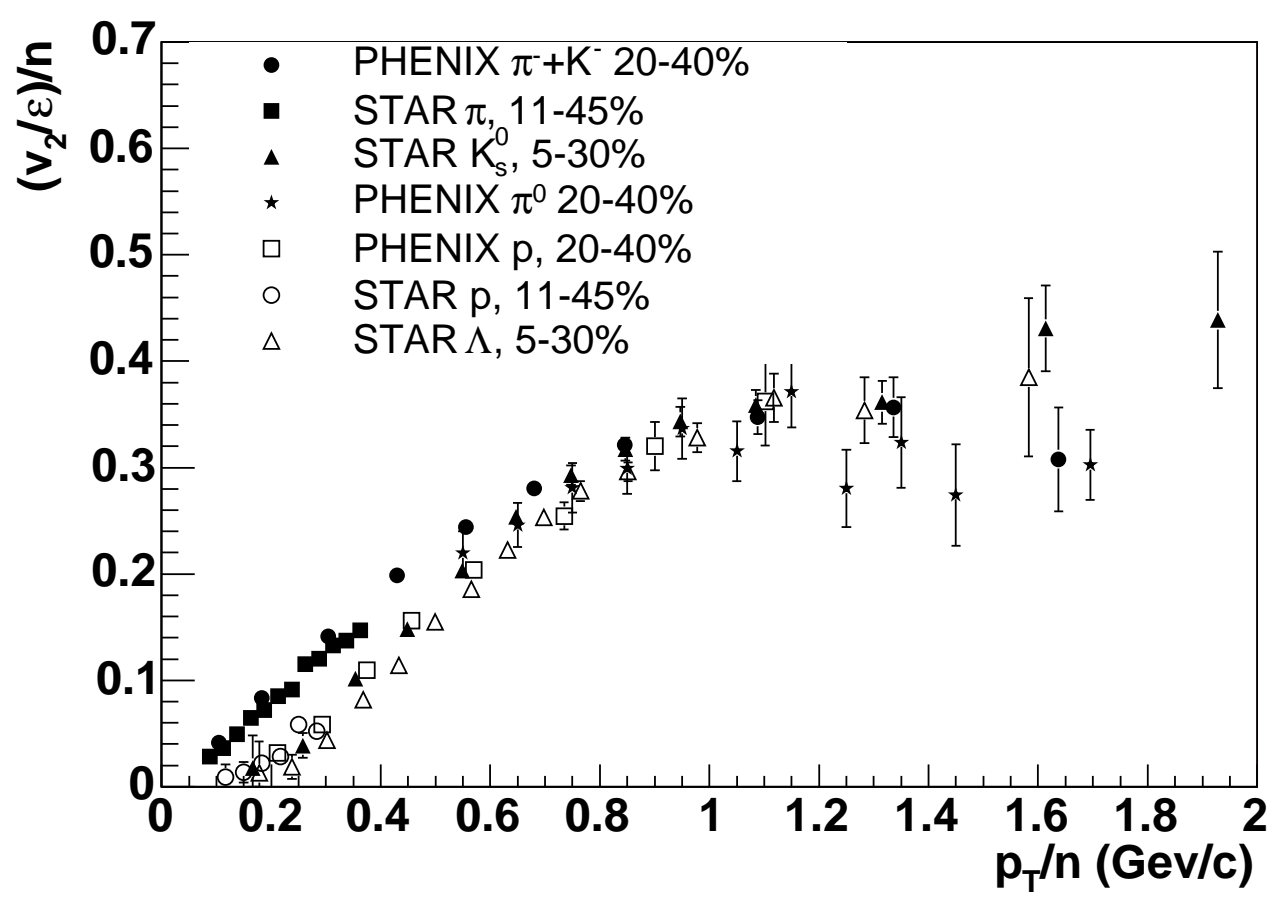

Fig. 55. $v_{2}$ as a function of transverse momentum for a variety of particles for $\mathrm{Au}+$ Au collisions where both $v_{2}$ and $p_{T}$ have been scaled by the number of constituent quarks in the particle. The meson data are shown with filled symbols; $\pi^{-}+K^{-}$ from PHENIX at $\sqrt{s_{N N}}=200 \mathrm{GeV}$ [50] (filled circles), charged $\pi$ from STAR at $\sqrt{s_{N N}}=130 \mathrm{GeV}[103]$ (filled squares), $K_{s}^{0}$ from STAR at $\sqrt{s_{N N}}=200 \mathrm{GeV}[239]$ (filled triangles), and $\pi^{0}$ from PHENIX at $\sqrt{s_{N N}}=200 \mathrm{GeV}$ [240] (filled stars). While the baryons are shown with open symbols; $p$ from PHENIX at $\sqrt{s_{N N}}=200$ $\mathrm{GeV}$ [50] (open squares), $p$ from STAR at $\sqrt{s_{N N}}=130 \mathrm{GeV}$ [103] (open circles), and $\Lambda$ from STAR at $\sqrt{s_{N N}}=200 \mathrm{GeV}$ [239] (open triangles).

decay of resonances such as the $\rho$. In this model, at least, the agreement of $v_{2}$ with the data is preserved - in addition a simple explanation is given for the excess of pion $v_{2}$ at low $p_{T}$. A similar conclusion in shown in [242]. This would seemingly attribute all the elliptic flow to the partonic phase leaving no room for additional flow to be produced in the later, hadronic stage - which may be in contradiction to hydrodynamic interpretations of the hadronic state as demanded by a variety of signatures such as the $p_{T}$ spectra of the protons and pions (see Section 3). It is clear that a more comprehensive comparison of observables should be undertaken to check the validity of these models. Higher-statistics jet studies with different identified particles by PHENIX in Run-4 will help clarify the situation. 


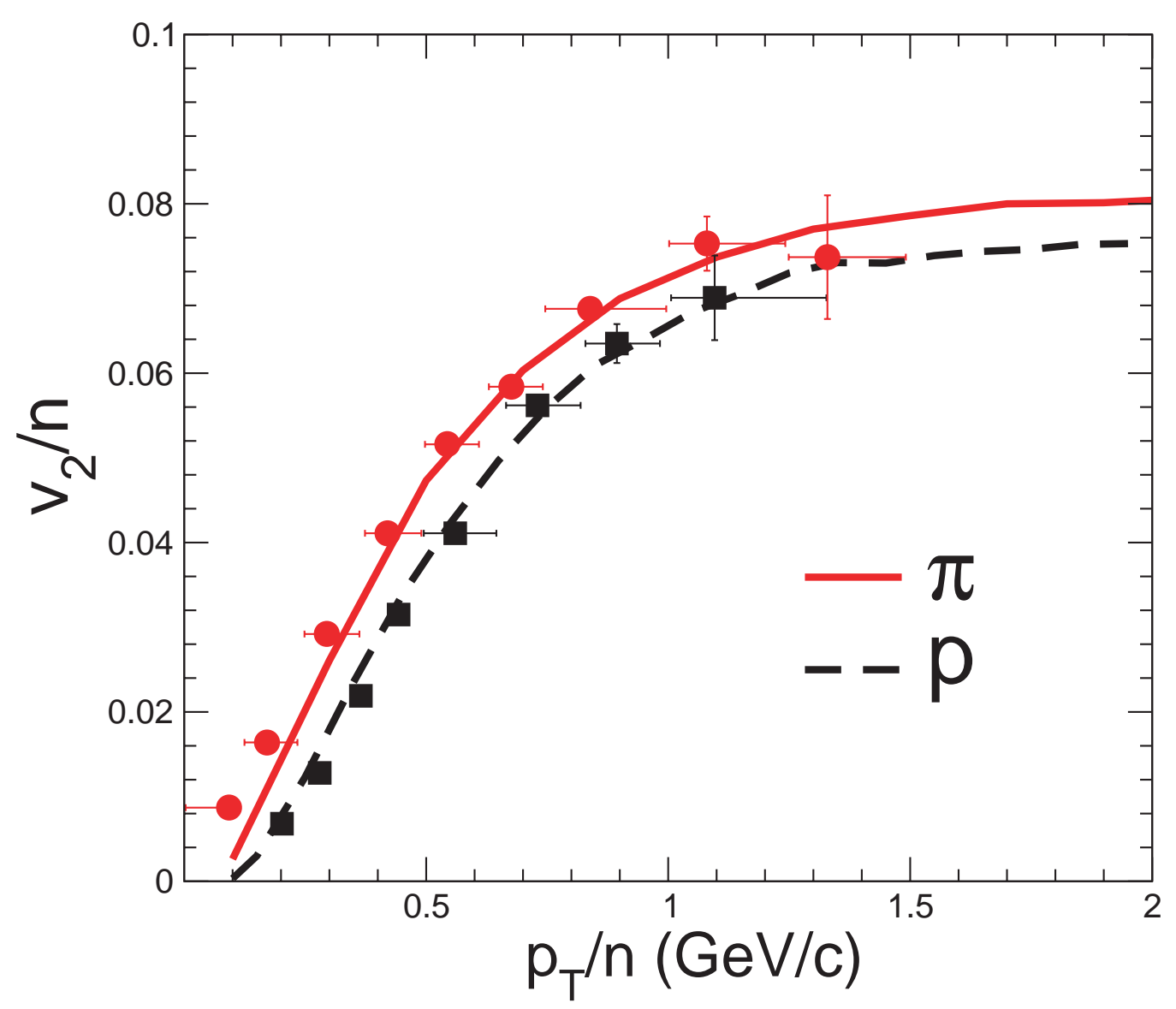

Fig. 56. $v_{2} / n$ in the TAMU model, where $\mathrm{n}$ is the number of constituent quarks in a particle for protons and pions[241]. Scaled pion (dashed line) and proton (dotted line) results from the TAMU model are shown in addition to charged pion and proton measurements from the PHENIX experiment from minimum bias $\mathrm{Au}+\mathrm{Au}$ reactions at $\sqrt{s_{N N}}=200 \mathrm{GeV}[50]$. This model allows for the recombination of hard partons and soft partons, as well as the decay of resonances such as the two pion decay of the $\rho$ meson. One sees that, at least in this calculation, the addition of processes which mix hard and soft partons do not destroy the agreement for the model with $v_{2} / n$ which is presumably a soft process.

\subsection{Hadron Formation Time}

In the discussion of the suppression of pions for $p_{T}>2 \mathrm{GeV} / c$, we treat the pions as resulting from the fragmentation of hard-scattered quarks and gluons. The explanation of this suppression in terms of partonic energy loss assumes that the hadronic wave function only becomes coherent outside the medium. Protons have a different hadronic structure and larger mass, and so may have a different, shorter time scale for coherence.

Following [220], we can estimate the formation time for the different mass hadrons at moderate $p_{T}$ in two different ways. According to the uncertainty 
principle, the formation time in the rest frame of the hadron can be related to the hadron size, $R_{h}$. In the laboratory frame, the hadron formation time is then given by

$$
\tau_{f} \approx R_{h} \frac{E_{h}}{m_{h}}
$$

where $R_{h}$ is taken to be $0.5-1 \mathrm{fm}$. For a $10 \mathrm{GeV} / c$ pion, this gives a formation time of $35-70 \mathrm{fm} / c$. For the $p_{T}=2.5 \mathrm{GeV} / c$ pions considered in this section, the formation time is $9-18 \mathrm{fm} / c$, well outside the collision region. However for $p_{T}=2.5 \mathrm{GeV} / c$ protons, the corresponding formation time is only $2.7 \mathrm{fm} / c$ in the vacuum, suggesting the possibility that the hadronization process may begin inside the medium. However the formation of such heavy particles would presumably be delayed in a deconfined medium until the entire system began to hadronize.

If quarks and antiquarks from gluon splitting are assumed to combine into dipole color singlets leading to the final hadrons, the formation time may be estimated from the gluon emission time. Then the formation time for a hadron carrying a fraction $z$ of the parton energy is given by

$$
\tau_{f} \approx \frac{2 E_{h}(1-z)}{k_{T}^{2}+m_{h}^{2}} .
$$

If $z$ is $0.6-0.8$ and $k_{T} \approx \Lambda_{Q C D}$, proton formation times in the range of 1-4 $\mathrm{fm}$ result [220]. Such values again imply formation of the proton within the medium. Thus, it is possible that differing (and perhaps complicated) interactions with the medium may produce different scalings of proton and pion production and result in modified fragmentation functions in $\mathrm{Au}+\mathrm{Au}$ collisions. However, most expectations are that this should lead to greater suppression rather than less. In fact, modified fragmentation functions measured in electron deep-inelastic scattering on nuclei by the HERMES experiment are often interpreted in terms of additional suppression for hadrons forming in the nuclear material.

\subsection{Hard-Scattering Physics}

If the dominant source of (anti)protons at intermediate $p_{T}$ is not soft physics, is the explanation a medium-modified hard-process source? The near-side partner yields indicate that a significant fraction of the baryons have jet-like partners. However, in the parton energy loss scenario as described in Section 6, hard-scattered partons lose energy in medium prior to hadronization. Thus one would expect the same suppression for baryons and mesons. Furthermore, 
we know that the (anti)protons are as suppressed above $p_{T}=5 \mathrm{GeV} / c$ in a manner similar to pions. Hence for this explanation to be correct, there must exist a mechanism by which only partons leading to baryons between 2 and 5 $\mathrm{GeV} / c$ in $p_{T}$ escape suppression.

Another key piece of information is that the elliptic flow $v_{2}$ for protons is large for $p_{T}$ in the range $2-4 \mathrm{GeV} / c$. At low $p_{T}$ this collective motion is attributed to different pressure gradients along and perpendicular to the impact parameter direction in semi-central collisions. At higher $p_{T}$ it has been hypothesized that one could observe a $v_{2}$ due to smaller partonic energy loss for partons traveling along the impact parameter direction (shorter path in the medium) as opposed to larger partonic energy loss in the perpendicular direction (larger path in the medium). However, the data suggest that the pions have a large energy loss (a factor of five suppression in central $\mathrm{Au}+\mathrm{Au}$ reactions), while the protons do not. In this case one might expect that if the source of proton $v_{2}$ were energy loss, then proton $v_{2}$ would be significantly less than the $v_{2}$ for the pions. In fact, the opposite is experimentally observed: for $p_{T}>2 \mathrm{GeV} / c$, the proton $v_{2}$ is always larger than the pion $v_{2}$.

The contradictions the data create for both the "soft"- and "hard"-physics explanations may indicate that the correct physics involves an interplay between the two.

\subsection{Conclusions}

The anomalous enhancement of (anti)protons relative to pions at intermediate $p_{T}=2-5 \mathrm{GeV}$ remains a puzzle. At lower transverse momentum particle production is a long-wavelength "soft" process and the transport of these hadrons and their precursor partons is reasonably described by hydrodynamics. As observed at lower energies, soft particles emitted from an expanding system receive a collective velocity boost to higher $p_{T}$ resulting in an enhanced $p / \pi$ and $\bar{p} / \pi$ ratio relative to proton-proton reactions at the same energy. We observe a similar phenomena at RHIC, for which the (anti) proton spectra and $v_{2}$ are roughly described in some hydrodynamic models up to approximately $2 \mathrm{GeV} / c$. Another class of calculations, referred to as recombination models, also boosts soft physics to higher $p_{T}$ by coalescence of "dressed" partons. In the hydrodynamic models the quanta which are flowing are initially partons and then hadrons. The recombination models describe comoving valence partons which coalesce into hadrons, and do not reinteract. These two points of view may not be entirely contradictory, since both include a flowing partonic phase. If fact, it may be that the recombination models provide a mechanism by which hydrodynamics works to a much higher $p_{T}$ than one might expect. The simplifying assumption of hadrons which do not interact is most probably 
an oversimplification and further refinement of the models will include this, though it may be that the hadronic phase will not modify the spectra as much as the hydrodynamic models might predict.

In both models, the (anti)proton enhancement as a function of centrality can be tuned to reproduce the apparent binary collision scaling observed in the data. An important distinction between the two is that in one case this enhancement is mass dependent and in the other it comes from the combination of quark momenta and thus distinguishes between baryons and mesons ${ }^{10}$. $R_{C P}$ for the $\phi$ is similar to other mesons despite the fact that they are more massive than protons. This scaling with quark content, as opposed to mass, favors recombination models.

Further investigations into these intermediate $p_{T}$ baryons reveals a near-angle correlation between particles, in a fashion characteristic of jet fragmentation. The near-angle associated particle yield increases by almost a factor of two in going from proton-proton and deuteron-gold reactions to gold-gold peripheral collisions. In addition, the partner yield is similar for trigger pions and protons, except in the most central gold-gold reactions. This appears to indicate a hard process source for a significant fraction of these baryons in contrast to the previous mentioned physics scenarios. Quantifying the precise contribution is an important goal for future measurements.

The large (anti) baryon to pion excess relative to expectations from parton fragmentation functions at intermediate $p_{T}=2-5 \mathrm{GeV} / c$ remains one of the most striking unpredicted experimental observations at RHIC. The data clearly indicate a new mechanism other than universal parton fragmentation as the dominant source of baryons and anti-baryons at intermediate $p_{T}$ in heavy ion collisions. The boosting of soft physics, that dominates hadron production at low $p_{T}$, to higher transverse momentum has been explored with the context of hydrodynamic and recombination models. However, investigations into these intermediate $p_{T}$ baryons reveals a near-angle correlation between particles, in a fashion characteristic of jet fragmentation. If instead these baryons have a partonic hard scattering followed by fragmentation source, this fragmentation process must be significantly modified. It is truly remarkable that these baryons have a large $v_{2}$ (typically 20\%) indicative of strong collective motion and also a large "jet-like" near-side partner yield. At present, no theoretical framework provides a complete understanding of hadron formation in the intermediate $p_{T}$ region.

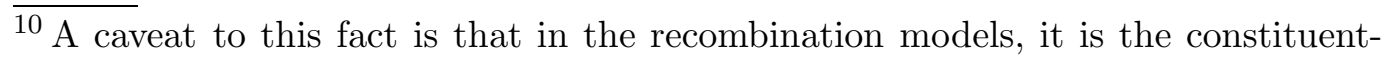
quark mass that is important, thereby giving a slightly larger mass to the strange quark.
} 


\section{FUTURE MEASUREMENTS}

The previous sections have documented the breadth and depth of the PHENIX data from the first three years of RHIC operations, along with the physics implications of those results. Here we describe those measurements required to further define and characterize the state of matter formed at RHIC. In particular, we note that the study of penetrating probes, which are the most sensitive tools in this endeavor, is just beginning. The PHENIX experiment was specifically designed to address these probes with capabilities that are unique within the RHIC program and unprecedented in the field of relativistic heavy ion physics.

One can distinguish two broad classes of penetrating probes:

(1) Hard probes created at the very early stage of the collision which propagate through, and could be modified by, the medium. These are the QCD hard-scattering probes and the main observables are high- $p_{T}$ particles coming from the fragmentation of jets, hidden charm $(J / \psi$ production), open charm and eventually also bottom quark and $\Upsilon$ production.

(2) Electromagnetic probes (either real or virtual photons) which are created by the medium. Due to their large mean free path these probes can leave the medium without final-state interaction thus carrying direct information about the medium's conditions and properties. The main observables here are low-mass $e^{+} e^{-}$pairs and the thermal radiation of the medium.

By their very nature, penetrating probes are also rare probes and consequently depend on the development of large values of the integrated luminosity. In the present data set the reach for high- $p_{T}$ particles in PHENIX extends to roughly $10 \mathrm{GeV} / c$, and lower-cross-section measurements such as charmonium are severely limited. The dramatic improvement of the machine performance in the year 2004 run provides confidence that both this data set and those from future RHIC runs will dramatically extend our reach in the rare probes sector.

As part of a decadal planning of the RHIC operation, PHENIX has prepared a comprehensive document that outlines in great detail its scientific goals and priorities for the next 10 years together with the associated detector upgrade program needed to achieve them. The decadal plan [243] is centered around the systematic study of the penetrating probes listed above. The program is broad and can accommodate additions or modifications provided that a compelling physics case can be made. Measurements are mainly planned in $\mathrm{Au}+\mathrm{Au}$ collisions at the full RHIC energy but they will be supplemented by other measurements varying the energy and/or the species and by the necessary reference measurements of $p+p$ and $p+A$ collisions. A short summary 
is given below.

\subsection{High-p $_{T}$ Suppression and Jet Physics}

The most exciting results to date at RHIC are the discovery of high- $p_{T}$ suppression of mesons, interpreted in terms of energy loss of quarks in a high-density medium, and the nonsuppression of baryons or equivalently, the anomalously high $p / \pi$ ratio which still awaits a clear explanation. These two topics were extensively discussed in Sections 6 and 7, respectively.

The data collected so far are superb. However, they suffer from limited reach in transverse momentum, limited particle identification capabilities and limited statistics in particular for detailed studies of jet correlations. PHENIX has a program for further studies of the high- $p_{T}$-suppression phenomena and jet physics which aims at overcoming these limitations.

It will be necessary to trace the suppression pattern to much higher $p_{T}$ to determine whether (and if so, when) the suppression disappears and normal perturbative QCD behavior sets in. High-luminosity runs will be needed, with at least a factor of 50 more statistics. PHENIX is particularly able to perform these measurements with its excellent capability of triggering on highmomentum $\pi^{0}$ 's.

PHENIX has performed several particle correlation analyses and has demonstrated that the experiment's aperture at mid-rapidity is sufficient to conduct these studies. Currently, these analyses are limited by the available statistics. Again, increasing the data sample by a factor of 50-100 will allow a variety of correlation studies using trigger particles with much-higher-momentum than studied to date. A particularly interesting case is the study of high-momentum $\gamma$-jet correlations, which have vastly reduced trigger bias, since the trigger photons propagate through the medium with a very long mean free path.

To further elucidate the baryon puzzle, additional data is required with better separation between baryons and mesons. An upgrade consisting of an aerogel Cerenkov counter and a high-resolution TOF detector is expected to be completed in time for the year 2006. A portion of this aerogel counter was already installed prior of the year 2004 run and performed according to expectations. Once completed, this high- $p_{T}$ detector will allow identification of $\pi, K / p$ to beyond $8 \mathrm{GeV} / c$ in $p_{T}$. 


\section{2 $J / \psi$ Production}

Suppression of heavy quarkonia is one of the earliest and most striking proposed signatures of deconfinement. The suppression mechanism follows directly from the Debye screening expected in the medium, which reduces the range of the potential between charm quark and anti-quark pairs [244]. The NA38 and NA50 experiments have carried out a systematic study of $J / \psi$ and $\psi^{\prime}$ at the CERN-SPS in $p+p, p+A$, light ion, and $\mathrm{Pb}+\mathrm{Pb}$ collisions providing some of the most intriguing results of the relativistic heavy ion program for more than ten years. The NA50 experiment observed an anomalous suppression of $J / \psi$ in central $\mathrm{Pb}+\mathrm{Pb}$ collisions at $\sqrt{s_{N N}}=17.2 \mathrm{GeV}$ [245]. The suppression, which is of the order of $25 \%$ with respect to the normal suppression in nuclear matter, has been interpreted by the NA50 authors as evidence for deconfinement of quarks and gluons. Although this interpretation is not universally shared $[246,247]$, the results of NA38 and NA50 demonstrate the utility and great interest in understanding the fate of charmonium in dense nuclear matter.

The theoretical expectations at RHIC energies are not at all clear. They range from total suppression in the traditional Debye screening scenario to enhancement in coalescence models $[248,249,250]$ and in statistical hadronization models $[251,252]$, of $c$ and $\bar{c}$ quarks. Although some versions of the coalescence model seem disfavored from our very limited data set [51], a more conclusive statement on these models has to await the much larger data set of the year 2004 run.

PHENIX has unprecedented capabilities for the study of the $J / \psi$ in $\mathrm{Au}+\mathrm{Au}$ collisions. The $J / \psi$ can be measured via its $\mu^{+} \mu^{-}$decay channel at forward and backward rapidities in the muon spectrometers and via its $e^{+} e^{-}$decay channel at mid-rapidity in the central arm spectrometers. From the recorded luminosity of the year 2004 run, we expect several thousand and $\sim 500 \mathrm{~J} / \psi$ in the muon and central arms, respectively. This data set will allow us a first look at the $J / \psi$ production pattern at RHIC. However, it could well be marginal for a complete characterization as a function of centrality and $p_{T}$, so that it is likely that further higher-luminosity runs will be required. Also the $p+p$ and $d+\mathrm{Au}$ baseline measurements performed in the year 2001 - 2003 runs have large statistical uncertainties, and higher-statistics versions for these colliding species will be needed. A high-luminosity $p+p$ run is planned in the year 2005 and high-luminosity $d+A$ or $p+A$ are still to be scheduled in the next years. 


\subsection{Charm Production}

Charm quarks are expected to be produced in the initial hard collisions between the incoming partons. The dominant mechanism is gluon fusion and thus the production cross section is sensitive to the gluon density in the initial state. The $c \bar{c}$ production cross section is sizable at RHIC energies with a few $c \bar{c}$ pairs and therefore several open charm mesons per unit of rapidity in central $\mathrm{Au}+\mathrm{Au}$ collisions. As a result, charm observables become readily accessible at RHIC and offer additional and extraordinarily valuable diagnostic tools. For example, it is vitally important to perform measurements of charm flow and to determine the energy loss of charm quarks in the medium. Such measurements will determine if the bulk dynamics observed for light quarks extend to charm quarks, which could in fact have very different behavior due to their much larger mass. Again the potential of PHENIX in this domain is unique with its capability of measuring open charm in a broad rapidity range, in the central and muon arms, via both the electron and muon decay channels. An additional unique feature is the possibility to measure correlated semileptonic charm decays by detecting $e-\mu$ coincidences from correlated $D \bar{D}$ decays. Such a measurement is particularly interesting for the study of charm-quark energy loss which may differ significantly from that observed for lighter quarks $[253,254,255]$. A first study of $e-\mu$ coincidences should be feasible with the year 2004 data.

To date PHENIX has measured charm production cross section in an indirect way through high- $p_{T}$ single electrons $[42,57]$ assuming that all electrons (after measuring and subtracting the contributions from light hadrons and photon conversions) originate from the semileptonic decays of charm quarks. Although the charm cross section has large uncertainties, the centrality dependence of the charm rapidity density demonstrates that charm production follows binary scaling as shown in Fig. 34. Improvements and additional information are expected from the much higher statistic of the year 2004 data.

A qualitatively new advance for PHENIX in the charm and also the beauty sector will be provided by the implementation of the silicon vertex detector. An upgrade project is underway to install in the next five years a silicon vertex tracker, including a central arm barrel and two end caps in front of the two muon spectrometers. The vertex tracker will allow us to resolve displaced vertices and therefore to directly identify open charm mesons via hadronic, e.g. $D \rightarrow K \pi$, as well as semi-leptonic decays. The heavy-quark physics topics accessible with the vertex tracker include production cross section and energy loss of open charm and open beauty, and spectroscopy of charmonium and bottomonium states, each of which should provide incisive new details on the properties of the created medium. 


\subsection{Low-Mass Dileptons}

Low-mass dileptons are considered the most sensitive probe of chiral symmetry restoration primarily through $\rho$ meson decays. Due to its very short lifetime $(\tau=1.3 \mathrm{fm} / c)$ compared to that of the typical fireball of $\sim 10 \mathrm{fm} / c$, most of the $\rho$ mesons decay inside the medium providing an unique tool to observe in-medium modifications of its properties (mass and/or width) which could be linked to chiral symmetry restoration. The situation is somewhat different but still interesting for the $\omega$ and $\phi$ mesons. Because of their much longer lifetimes $(\tau=23 \mathrm{fm} / c$ and $46 \mathrm{fm} / c$ for the $\omega$ and $\phi$, respectively ) they predominantly decay outside the medium, after regaining their vacuum properties, with only a small fraction decaying inside the medium. Since the measurement integrates over the history of the collision, this may result in a small modification of the line shape of these two mesons which PHENIX might be able to observe with its excellent mass resolution. PHENIX also has the unprecedented capability of simultaneously measuring within the same apparatus the $\phi$ meson decay through $e^{+} e^{-}$and $K^{+} K^{-}$channels. The comparison of the branching ratios to these two channels provides a very sensitive tool for in-medium modifications of the $\phi$ and $K$ mesons.

The CERES experiment at CERN has confirmed the unique physics potential of low-mass dileptons $[256,257,258]$. An enhancement of electron pairs was observed in the mass region $m=0.2-0.6 \mathrm{GeV} / c^{2}$ in $\mathrm{Pb}+\mathrm{Au}$ collisions at $\sqrt{s_{N N}}=$ 17.2 $\mathrm{GeV}$ with respect to $p+p$ collisions. The results have triggered a wealth of theoretical activity and can be explained by models which invoke in-medium modification of the $\rho$ meson (dropping of its mass and/or broadening of its width) [259]. The precision of the CERES data has been so far insufficient to distinguish between the different models. Results with higher statistics and better mass resolution are expected from the NA60 experiment that is studying the production of low-mass dimuons in In+In collisions [260]. Theoretical calculations [261] show that the enhancement should persist at RHIC energies and that PHENIX with its excellent mass resolution has an unique opportunity to do precise spectroscopy of the light vector mesons and to shed more light on the origin of the enhancement of the low-mass-pair continuum.

The measurement of low-mass electron pairs is however a very challenging one. The main difficulty stems from the huge combinatorial background created by the pairing of $e^{+}$and $e^{-}$tracks from unrecognized $\pi^{0}$ Dalitz decays and $\gamma$ conversions. PHENIX is developing a novel Cerenkov detector that, in combination with the recently installed coil which makes the magnetic field zero close to the beam axis, will effectively reduce this combinatorial background by almost two orders of magnitude [262]. The detector, operated in pure $\mathrm{CF}_{4}$, consists of a 50-cm-long radiator directly coupled, in a windowless configuration, to a triple GEM detector which has a CsI photocathode evap- 
orated on the top face of the first GEM foil and pad read out at the bottom of the GEM stack [263]. The R\&D phase to demonstrate the validity of the concept is nearing completion. The detector construction phase is starting now with installation foreseen in time for the year 2006 - 2007. With this detector PHENIX will have the unprecedented ability to perform high-quality measurements over the whole dilepton mass range from the $\pi^{0}$ Dalitz decay up to the charmonium states.

\subsection{Thermal Radiation}

A prominent topic of interest in the field of relativistic heavy-ion collisions is the identification of the thermal radiation emitted by the system and in particular the thermal radiation emitted by the quark-gluon plasma via $q \bar{q}$ annihilation. Such radiation is a direct fingerprint of the matter formed and is regarded as a very strong signal of deconfinement. Its spectral shape should provide a direct measurement of the plasma temperature.

In principle the thermal radiation can be studied through real photons or dileptons, since real and virtual photons carry basically the same physics message. In practice the measurements are extremely challenging. The thermal radiation is expected to be a small signal compared to the large background from competing processes, hadron decays for real photons and Dalitz decays and $\gamma$ conversions for dileptons, the former being larger by orders of magnitude compared to the latter. But in both cases, a very precise knowledge of all these sources is an absolutely necessary prerequisite. After subtracting these sources, one still needs to disentangle other contributions which might be comparable or even stronger, mainly the contributions of initial hard-parton scattering to direct photons and of semileptonic decays of charm mesons to dileptons.

Theoretical calculations have singled out the dilepton mass range $m=1$ $3 \mathrm{GeV} / c^{2}$ as the most appropriate window where the QGP radiation could dominate over other contributions [264,265]. Measurements in this intermediate mass range carried out at the CERN SPS by HELIOS and NA50 have revealed an excess of dileptons, but this excess could be explained by hadronic contributions [266].

There is no conclusive evidence for QGP thermal photons from the CERN experiments (for a recent review see [267]). From the theoretical point of view it is clear that in the low- $p_{T}$ region $\left(p_{T}<2 \mathrm{GeV} / c\right)$ the real photon spectrum is dominated by hadronic sources and the thermal radiation from the hadron gas. It is only in the high- $p_{T}$ region where one might have a chance to observe the thermal radiation from the QGP. 
PHENIX has measured direct real photons at $p_{T}>4 \mathrm{GeV} / c$ from the initial hard scatterings[73]. The errors are relatively large leaving room for a comparable contribution of thermal photons. The high statistics of the year 2004 run will provide the first real opportunity to search for the QGP thermal radiation in PHENIX both in the dilepton and real photon channels. However, the search for this elusive signal might take some time as it will probably require equally-high-statistics runs of reference data in $p+p$ and $p+A$ collisions for a precise mapping of all the other contributions (hadronic + pQCD for real photons and hadronic + charm for dileptons).

\section{SUMMARY AND CONCLUSIONS}

The PHENIX data set from the first three years of RHIC operation provides an extensive set of measurements, from global variables to hadron spectra to high$p_{T}$ physics to heavy-flavor production. From this rich menu we have reviewed those aspects of the present data that address the broad features of the matter created in $\mathrm{Au}+\mathrm{Au}$ collisions at RHIC, namely, energy and number density, thermalization, critical behavior, hadronization, and possible deconfinement.

We first investigated whether the transverse energy and multiplicity measurements of PHENIX demonstrate that a state of high-energy-density matter is formed in $\mathrm{Au}+\mathrm{Au}$ collision at RHIC. We estimated from our $d E_{T} / d \eta$ measurement that the peak energy density in the form of created secondary particles is at least $15 \mathrm{GeV} / \mathrm{fm}^{3}$. If we use a thermalization time of $1 \mathrm{fm} / c$ provided by the hydrodynamic models from the elliptic flow, then the value of the energy density of the first thermalized state would be in excess of $5 \mathrm{GeV} / \mathrm{fm}^{3}$. These values are well in excess of the $\sim 1 \mathrm{GeV} / \mathrm{fm}^{3}$ obtained in lattice QCD as the energy density needed to form a deconfined phase. Nä̈ve expectations prior to RHIC turn-on that $d E_{T} / d \eta$ and $d N_{c h} / d \eta$ could be factorized into a "soft" and a pQCD jet component are not supported by the data. Results from a new class of models featuring initial-state gluon saturation compare well with RHIC multiplicity and $E_{T}$ data.

We then examined our data and various theoretical models to investigate the degree to which the matter formed at RHIC appears to be thermalized. The measured yields and spectra of hadrons are consistent with thermal emission from a strongly expanding source, and the observed strangeness production is consistent with predictions based on complete chemical equilibrium. The scaling of the strength of the elliptic flow $v_{2}$ with eccentricity shows that a high degree of collectivity is built up at a very early stage of the collision. The hydro models which include both hadronic and QGP phases reproduce the qualitative features of the measured $v_{2}\left(p_{T}\right)$ of pions, kaons, and protons. These hydro models require early thermalization $\left(\tau_{\text {therm }} \leq 1 \mathrm{fm} / c\right)$ and high 
initial energy density $\varepsilon \geq 10 \mathrm{GeV} / \mathrm{fm}^{3}$. These points of agreement between the data and the hydrodynamic and thermal models can be interpreted as strong evidence for formation of high-density matter that thermalizes very rapidly.

However several of the hydro models fail to reproduce the $v_{2}\left(p_{T}\right)$ of pions, protons, and spectra of pions and protons simultaneously. Given this disagreement it is not yet possible to make an unequivocal statement regarding the presence of a QGP phase based on comparisons to hydrodynamic calculations. The experimentally measured HBT source parameters, especially the small value of $R_{\text {long }}$ and the ratio $R_{\text {out }} / R_{\text {side }} \approx 1$, are not reproduced by the hydrodynamic calculations. Hence we currently do not have a consistent picture of the space-time dynamics of reactions at RHIC as revealed by spectra, $v_{2}$ and HBT. These inconsistencies prevent us from drawing firm conclusions on properties of the matter such as the equation of state and the presence of a mixed phase.

Critical behavior near the phase boundary can produce nonstatistical fluctuations in observables such as the net-charge distribution and the average transverse momentum. Our search for charge fluctuations has ruled out the most nä̈e model of charge fluctuations in a QGP, but it is unclear if the charge fluctuation signature can survive hadronization. Our measurement of $\left\langle p_{T}\right\rangle$ fluctuations is consistent with the effect expected of high- $p_{T}$ jets, and it gives a severe constraint on the fluctuations that were expected for a sharp phase transition.

Many of these observables - for instance, large $d E / d \eta$ and $d N_{c h} / d \eta$, strangeness enhancement, strong radial flow, and elliptic flow-have been observed in heavy ion collisions at lower energies. We have found smooth changes in these observables as a function of $\sqrt{s_{N N}}$ from AGS energies to SPS energies to RHIC energy. The $d E_{T} / d \eta$ increases by about $100 \%$ and the strength of the elliptic flow increase by about $50 \%$ from SPS to RHIC. The strangeness suppression factor $\gamma_{s}$ and the radial expansion velocity $\left\langle\beta_{T}\right\rangle$ vary smoothly from AGS to RHIC energies. No sudden change with collision energy has been observed.

The strong suppression of high- $p_{T}$ particle production at RHIC is a unique phenomenon that has not been previously observed. Measurements of two-hadron azimuthal-angle correlations at high $p_{T}$ and the $x_{T}$ scaling in $\mathrm{Au}+\mathrm{Au}$ collisions confirm the dominant role of hard scattering and subsequent jet fragmentation in the production of high- $p_{T}$ hadrons. Measurements in deuteron-gold collisions demonstrate that any initial-state modification of nuclear parton distributions causes little or no suppression of hadron production for $p_{T}>2$ $\mathrm{GeV} / c$ at mid-rapidity. This conclusion is further strengthened by the observed binary scaling of direct photon and open charm yields in $\mathrm{Au}+\mathrm{Au}$. Combined together, these observations provide direct evidence that $\mathrm{Au}+\mathrm{Au}$ collisions at RHIC have produced matter at extreme densities. 
Medium-induced energy loss, predominantly via gluon bremsstrahlung emission, is the only currently known physical mechanism that can fully explain the magnitude of the observed high- $p_{T}$ suppression. The approximately flat suppression factor $R_{A A}\left(p_{T}\right)$ observed in the data, which was predicted by the GLV energy loss model, rules out the simplest energy loss models which predicted a constant energy loss per unit length. However, the model by Wang et al. obtains the same flat $R_{A A}\left(p_{T}\right)$ from apparently different physics. From the GLV model, the initial gluon number density, $d n_{g} / d y \approx 1000$ and initial energy density, $\varepsilon_{0} \approx 15 \mathrm{GeV} / \mathrm{fm}^{3}$, have been obtained. These values are consistent with the energy density obtained from our $d E_{T} / d \eta$ measurement as well as ones from the hydro models.

The large (anti)baryon to pion excess relative to expectations from parton fragmentation functions at intermediate $p_{T}(2-5 \mathrm{GeV} / c)$ is both an unpredicted and one of the most striking experimental observation at RHIC. The data clearly indicates that a mechanism other than universal parton fragmentation is the dominant source of (anti-)baryons in the intermediate $p_{T}$ range in heavy ion collisions. The boosting of soft physics to higher transverse momentum has been explored within the context of hydrodynamics and recombination models. Hydrodynamic models can readily explain the baryon to meson ratio as a consequence of strong radial flow, but these models have difficulties reproducing the difference in $v_{2}$ between protons and mesons above $2 \mathrm{GeV} / c$. Recombination models provide a natural explanation for the large baryon to meson ratio as well as the apparent quark-number scaling of the elliptic flow. However, investigations into these intermediate $p_{T}$ baryons reveal a near-angle correlation between particles, in a fashion characteristic of jet fragmentation. If instead these baryons have a partonic hard scattering followed by fragmentation, this fragmentation process must be significantly modified. It is truly remarkable that these baryons have a large $v_{2}$ of $\approx 20 \%$ typically indicative of strong collective motion and also a large jet-like nearside partner yield. At present, no model provides a complete understanding of hadron formation in the intermediate $p_{T}$ regime.

The initial operation of RHIC has produced the impressive quantity of significant results described above. These striking findings call for additional efforts to define, clarify and characterize the state of matter formed at RHIC. Further study of the collisions using hard probes such as high- $p_{T}$ particles, open charm, and $J / \psi$, and electromagnetic probes such as direct photons, thermal photons, thermal dileptons, and low-mass lepton pairs are particularly important. The utilization of these penetrating probes is just beginning, and we expect these crucial measurements based on the very-high-statistics data of the year 2004 run will provide essential results towards understanding of the dense matter created at RHIC.

Advances in the theoretical understanding of relativistic heavy ion collisions 
is vital for the quantitative study of the dense matter formed at RHIC. While there is rapid and significant progress in this area, a coherent and consistent picture of heavy ion collisions at RHIC, from the initial formation of the dense matter to the thermalization of the system to the hadronization to the freezeout, remains elusive. With such a consistent model, it will become possible to draw definitive conclusions on the nature of the matter and to quantitatively determine its properties. The comprehensive data sets from global variables to penetrating probes provided by PHENIX at present and in the future will prove essential in constructing and constraining a consistent model of heavy ion collisions to determine the precise nature of the matter created at RHIC.

In conclusion, there is compelling experimental evidence that heavy-ion collisions at RHIC produce a state of matter characterized by very high energy densities, density of unscreened color charges ten times that of a nucleon, large cross sections for the interaction between strongly interacting particles, strong collective flow, and early thermalization. Measurements indicate that this matter modifies jet fragmentation and has opacity that is too large to be explained by any known hadronic processes. This state of matter is not describable in terms of ordinary color-neutral hadrons, because there is no known self-consistent theory of matter composed of ordinary hadrons at the measured densities. The most economical description is in terms of the underlying quark and gluon degrees of freedom. Models taking this approach have scored impressive successes in explaining many, but not all, of the striking features measured to date. There is not yet irrefutable evidence that this state of matter is characterized by quark deconfinement or chiral symmetry restoration, which would be a direct indication of quark-gluon plasma formation. The anticipated program of additional incisive experimental measurements combined with continued refinement of the theoretical description is needed to achieve a complete understanding of the state of matter created at RHIC.

\section{ACKNOWLEDGEMENTS}

We thank the staff of the Collider-Accelerator and Physics Departments at Brookhaven National Laboratory and the staff of the other PHENIX participating institutions for their vital contributions. We acknowledge support from the Department of Energy, Office of Science, Nuclear Physics Division, the National Science Foundation, Abilene Christian University Research Council, Research Foundation of SUNY, and Dean of the College of Arts and Sciences, Vanderbilt University (U.S.A), Ministry of Education, Culture, Sports, Sci-

ence, and Technology and the Japan Society for the Promotion of Science (Japan), Conselho Nacional de Desenvolvimento Científico e Tecnológico and Fundaç ao de Amparo à Pesquisa do Estado de S ao Paulo (Brazil), Natural 
Science Foundation of China (People's Republic of China), Centre National de la Recherche Scientifique, Commissariat à l'Énergie Atomique, Institut National de Physique Nucléaire et de Physique des Particules, and Association pour la Recherche et le Développement des Méthodes et Processus Industriels (France), Ministry of Industry, Science and Tekhnologies, Bundesministerium für Bildung und Forschung, Deutscher Akademischer Austausch Dienst, and Alexander von Humboldt Stiftung (Germany), Hungarian National Science Fund, OTKA (Hungary), Department of Atomic Energy and Department of Science and Technology (India), Israel Science Foundation (Israel), Korea Research Foundation and Center for High Energy Physics (Korea), Russian Ministry of Industry, Science and Tekhnologies, Russian Academy of Science, Russian Ministry of Atomic Energy (Russia), VR and the Wallenberg Foundation (Sweden), the U.S. Civilian Research and Development Foundation for the Independent States of the Former Soviet Union, the US-Hungarian NSF-OTKA-MTA, and the US-Israel Binational Science Foundation.

\section{References}

[1] G. F. Chapline, M. H. Johnson, E. Teller, M. S. Weiss, Phys. Rev. D8 (1973) 4302.

[2] T. D. Lee, G. C. Wick, Phys. Rev. D9 (1974) 2291.

[3] T. D. Lee, Rev. Mod. Phys. 47 (1975) 267.

[4] T. D. Lee, cU-TP-170, Published in Trans.N.Y.Acad.Sci.Ser.2 v.40 1980:0111. (1979).

[5] J. C. Collins, M. J. Perry, Phys. Rev. Lett. 34 (1975) 1353.

[6] N. Itoh, Prog. Theor. Phys. 44 (1970) 291.

[7] E. V. Shuryak, Phys. Rept. 61 (1980) 71.

[8] M. Creutz, Phys. Rev. D15 (1977) 1128.

[9] K. G. Wilson, Phys. Rev. D10 (1974) 2445.

[10] E. Laermann, O. Philipsen, Ann. Rev. Nucl. Part. Sci. 53 (2003) 163.

[11] F. Karsch, Lect. Notes Phys. 583 (2002) 209.

[12] K. Rajagopal, Acta Phys. Polon. B31 (2000) 3021.

[13] Z. Fodor, S. D. Katz, JHEP 0404 (2004) 050.

[14] S. Ejiri, et al., Prog. Theor. Phys. Suppl. 153 (2004) 118.

[15] W. Greiner, S. Schramm, E. Stein, Quantum Chromodynamics, 2nd Edition, Springer-Verlag, 2002. 
[16] V. V. Klimov, Sov. Phys. JETP 55 (1982) 199.

[17] H. A. Weldon, Phys. Rev. D26 (1982) 1394.

[18] S. Datta, F. Karsch, P. Petreczky, I. Wetzorke, Nucl. Phys. Proc. Suppl. 119 (2003) 487.

[19] M. Asakawa, T. Hatsuda, Phys. Rev. Lett. 92 (2004) 012001.

[20] S. Datta, F. Karsch, P. Petreczky, I. Wetzorke, Phys. Rev. D69 (2004) 094507.

[21] F. Karsch, et al., Nucl. Phys. A715 (2003) 701.

[22] E. V. Shuryak, I. Zahed, Phys. Rev. D70 (2004) 054507.

[23] B. Müller, we wish to acknowledge B. Müller for this observation. (2004).

[24] E. Shuryak, Prog. Part. Nucl. Phys. 53 (2004) 273.

[25] M. Gyulassy, L. McLerran, Nucl. Phys. A750 (2005) 30.

[26] G. Baym, in Proceedings of the Bielefeld Workshop on Quark Matter Formation and Heavy Ion Collisions, M. Jacob and H. Satz, ed. (May, 1982). (1982).

[27] M. Plümer, S. Raha, R. Weiner, Nucl. Phys. A418 (1984) 549c.

[28] B. Müller, nucl-th/9211010 (1992).

[29] K. Kajantie, M. Laine, K. Rummukainen, Y. Schroder, Phys. Rev. D67 (2003) 105008.

[30] A. D. Linde, Phys. Lett. B96 (1980) 289.

[31] D. J. Gross, R. D. Pisarski, L. G. Yaffe, Rev. Mod. Phys. 53 (1981) 43.

[32] Bear-Mountain, nearly the entire history of the various experimental efforts is documented in the proceedings of the various "Quark Matter" conferences, which began in 1981. A seminal document in the history of the field is the report of the "Bear Mountain" conference: Report of the workshop on BeV/nucleon collisions of heavy ions-how and why, Bear Mountain, New York, Nov. 29-Dec. 1, 1974 (BNL-AUI, 1975), (1974).

[33] G. Baym, Nucl. Phys. A698 (2002) XXIII.

[34] NSAC, 2002 NSAC Long-Range Plan: Opportunities in Nuclear Science, A Long-Range Plan for the Next Decade (April 2002). Available from http://www.sc.doe.gov/henp/np/nsac/nsac.html, (2002).

[35] PHENIX Collaboration,

PHENIX

$\equiv$ Pioneering High Energy Nuclear Interaction eXperiment. Full details are available from http://www.phenix.bnl.gov/ . (2004).

[36] PHENIX, pHENIX Conceptual Design Report 1993 (PX20, BNL48922, internal report), (1993). 
[37] K. Adcox, et al., Phys. Rev. Lett. 86 (2001) 3500.

[38] K. Adcox, et al., Phys. Rev. Lett. 87 (2001) 052301.

[39] K. Adcox, et al., Phys. Rev. Lett. 88 (2002) 022301.

[40] K. Adcox, et al., Phys. Rev. Lett. 88 (2002) 242301.

[41] K. Adcox, et al., Phys. Rev. Lett. 88 (2002) 192302.

[42] K. Adcox, et al., Phys. Rev. Lett. 88 (2002) 192303.

[43] K. Adcox, et al., Phys. Rev. Lett. 89 (2002) 092302.

[44] K. Adcox, et al., Phys. Rev. Lett. 89 (2002) 082301.

[45] K. Adcox, et al., Phys. Rev. C 66 (2002) 024901.

[46] K. Adcox, et al., Phys. Rev. Lett. 89 (2002) 212301.

[47] K. Adcox, et al., Phys. Lett. B561 (2003) 82.

[48] K. Adcox, et al., Phys. Rev. C69 (2004) 024904.

[49] S. S. Adler, et al., Phys. Rev. Lett. 91 (2003) 072301.

[50] S. S. Adler, et al., Phys. Rev. Lett. 91 (2003) 182301.

[51] S. S. Adler, et al., Phys. Rev. C69 (2004) 014901.

[52] S. S. Adler, et al., Phys. Rev. Lett. 91 (2003) 172301.

[53] S. S. Adler, et al., Phys. Rev. C69 (2004) 034910.

[54] S. S. Adler, et al., Phys. Rev. C69 (2004) 034909.

[55] S. S. Adler, et al., Phys. Rev. Lett. 93 (2004) 092301.

[56] S. S. Adler, et al., Phys. Rev. Lett. 93 (2004) 152302.

[57] S. S. Adler, et al., Phys. Rev. Lett. 94 (2004) 082301.

[58] S. S. Adler, et al., nucl-ex/0406004, accepted in Phys. Rev. Lett. (2004).

[59] S. S. Adler, et al., nucl-ex/0409015, accepted in Phys. Rev. C (2004).

[60] S. S. Adler, et al., Phys. Rev. Lett. 91 (2003) 241803.

[61] S. S. Adler, et al., Phys. Rev. Lett. 92 (2004) 051802.

[62] S. S. Adler, et al., Phys. Rev. Lett. 93 (2004) 202002.

[63] S. S. Adler, et al., hep-ex/0502006, accepted in Phy. Rev. D (2005).

[64] S. S. Adler, et al., Phys. Rev. Lett. 91 (2003) 072303.

[65] S. S. Adler, et al., Phys. Rev. Lett. 94 (2004) 082302. 
[66] I. Arsene, et al., Phys. Rev. Lett. 91 (2003) 072305.

[67] B. B. Back, et al., Phys. Rev. Lett. 91 (2003) 072302.

[68] J. Adams, et al., Phys. Rev. Lett. 91 (2003) 072304.

[69] S. S. Adler, et al., nucl-ex/0410012 (2004).

[70] S. S. Adler, et al., nucl-ex/0411040 (2004).

[71] S. S. Adler, et al., nucl-ex/0408007 (2004).

[72] S. S. Adler, et al., nucl-ex/0502009 (2005).

[73] S. S. Adler, et al., nucl-ex/0503003 (2005).

[74] J. D. Bjorken, Phys. Rev. D27 (1983) 140.

[75] L. Ahle, et al., Phys. Lett. B332 (1994) 258.

[76] T. Alber, et al., Phys. Rev. Lett. 75 (1995) 3814.

[77] A. Krasnitz, Y. Nara, R. Venugopalan, Nucl. Phys. A717 (2003) 268.

[78] P. F. Kolb, J. Sollfrank, U. W. Heinz, Phys. Rev. C62 (2000) 054909.

[79] S.-y. Li, X.-N. Wang, Phys. Lett. B527 (2002) 85.

[80] B. B. Back, et al., Phys. Rev. Lett. 85 (2000) 3100.

[81] X.-N. Wang, M. Gyulassy, Phys. Rev. Lett. 86 (2001) 3496.

[82] V. Topor Pop, et al., Phys. Rev. C68 (2003) 054902.

[83] D. Kharzeev, M. Nardi, Phys. Lett. B507 (2001) 121.

[84] D. Kharzeev, E. Levin, Phys. Lett. B523 (2001) 79.

[85] K. J. Eskola, K. Kajantie, P. V. Ruuskanen, K. Tuominen, Nucl. Phys. B570 (2000) 379.

[86] K. J. Eskola, P. V. Ruuskanen, S. S. Rasanen, K. Tuominen, Nucl. Phys. A696 (2001) 715 .

[87] F. Becattini, Z. Phys. C69 (1996) 485.

[88] F. Becattini, U. W. Heinz, Z. Phys. C76 (1997) 269 (e).

[89] P. Braun-Munzinger, K. Redlich, J. Stachel, nucl-th/0304013 (2003).

[90] M. Kaneta, N. Xu, nucl-th/0405068 (2004).

[91] J. Cleymans, B. Kampfer, M. Kaneta, N. Wheaton, S.and Xu, hep-ph/0409071 (2004).

[92] J. Adams, et al., Phys. Rev. Lett. 92 (2004) 182301. 
[93] P. Braun-Munzinger, D. Magestro, K. Redlich, J. Stachel, Phys. Lett. B518 (2001) 41.

[94] D. Magestro, J. Phys. G28 (2002) 1745.

[95] F. Becattini, M. Gazdzicki, A. Keranen, J. Manninen, R. Stock, Phys. Rev. C69 (2004) 024905.

[96] E. Schnedermann, J. Sollfrank, U. W. Heinz, Phys. Rev. C48 (1993) 2462.

[97] G. Rai, Nucl. Phys. A681 (2001) 181.

[98] H. Dobler, J. Sollfrank, U. W. Heinz, Phys. Lett. B457 (1999) 353.

[99] C. Alt, et al., J. Phys. G30 (2004) S119.

[100] T. Peitzmann, Eur. Phys. J. C26 (2003) 539.

[101] M. M. Aggarwal, et al., Phys. Rev. C67 (2003) 014906.

[102] J. Adams, et al., Phys. Rev. Lett. 92 (2004) 112301.

[103] C. Adler, et al., Phys. Rev. Lett. 87 (2001) 182301.

[104] C. Alt, et al., Phys. Rev. C68 (2003) 034903.

[105] G. Agakichiev, et al., Phys. Rev. Lett. 92 (2004) 032301.

[106] D. Teaney, J. Lauret, E. V. Shuryak, nucl-th/0110037 (2001).

[107] P. Huovinen, P. F. Kolb, U. W. Heinz, P. V. Ruuskanen, S. A. Voloshin, Phys. Lett. B503 (2001) 58.

[108] D. H. Rischke, M. Gyulassy, Nucl. Phys. A597 (1996) 701.

[109] G. F. Bertsch, Nucl. Phys. A498 (1989) 173c.

[110] S. Pratt, Phys. Rev. D33 (1986) 1314.

[111] S. Pratt, Phys. Rev. Lett. 53 (1984) 1219.

[112] A. N. Makhlin, Y. M. Sinyukov, Z. Phys. C39 (1988) 69.

[113] S. Pratt, T. Csoergoe, J. Zimanyi, Phys. Rev. C42 (1990) 2646.

[114] J. Adams, et al., Phys. Rev. Lett. 93 (2004) 012301.

[115] F. Retiere, J. Phys. G30 (2004) S827.

[116] M. Csanad, T. Csorgo, B. Lorstad, A. Ster, J. Phys. G30 (2004) S1079.

[117] P. F. Kolb, R. Rapp, Phys. Rev. C67 (2003) 044903.

[118] T. Hirano, K. Tsuda, Phys. Rev. C66 (2002) 054905.

[119] T. Hirano, Y. Nara, Nucl. Phys. A743 (2004) 305.

[120] P. F. Kolb, U. Heinz, nucl-th/0305084 (2003). 
[121] D. Molnar, P. Huovinen, Phys. Rev. Lett. 94 (2005) 012302.

[122] D. A. Teaney, J. Phys. G30 (2004) S1247.

[123] U. W. Heinz, P. F. Kolb, hep-ph/0204061 (2002).

[124] Y. M. Sinyukov, S. V. Akkelin, Y. Hama, Phys. Rev. Lett. 89 (2002) 052301.

[125] F. Grassi, Y. Hama, S. S. Padula, J. Socolowski, Octavio, Phys. Rev. C62 (2000) 044904.

[126] F. Cooper, G. Frye, Phys. Rev. D10 (1974) 186.

[127] S. Soff, S. A. Bass, A. Dumitru, Phys. Rev. Lett. 86 (2001) 3981.

[128] D. Zschiesche, S. Schramm, H. Stocker, W. Greiner, Phys. Rev. C65 (2002) 064902.

[129] F. Karsch, E. Laermann, hep-lat/0305025 (2003).

[130] S. Soff, hep-ph/0202240 (2002).

[131] G. N. Fowler, R. M. Weiner, Phys. Lett. B70 (1977) 201.

[132] G. N. Fowler, E. M. Friedlander, R. M. Weiner, Phys. Lett. B104 (1981) 239.

[133] L. Van Hove, Physica 147A (1987) 19.

[134] G. J. Alner, et al., Phys. Lett. B160 (1985) 193.

[135] S. Jeon, V. Koch, Phys. Rev. Lett. 85 (2000) 2076.

[136] M. Asakawa, U. W. Heinz, B. Muller, Phys. Rev. Lett. 85 (2000) 2072.

[137] H. Heiselberg, A. D. Jackson, Phys. Rev. C63 (2001) 064904.

[138] R. Albrecht, et al., Z. Phys. C45 (1989) 31.

[139] P. Carruthers, C. C. Shih, Phys. Lett. B165 (1985) 209.

[140] H. Sorge, Phys. Rev. C52 (1995) 3291.

[141] M. J. Tannenbaum, Phys. Lett. B498 (2001) 29.

[142] R. Korus, S. Mrowczynski, M. Rybczynski, Z. Wlodarczyk, Phys. Rev. C64 (2001) 054908.

[143] J. T. Mitchell, J. Phys. G30 (2004) S819.

[144] E. G. Ferreiro, F. del Moral, C. Pajares, Phys. Rev. C69 (2004) 034901.

[145] S. Gavin, Phys. Rev. Lett. 92 (2004) 162301.

[146] J. F. Owens, Rev. Mod. Phys. 59 (1987) 465.

[147] G. Bunce, N. Saito, J. Soffer, W. Vogelsang, Ann. Rev. Nucl. Part. Sci. 50 (2000) 525. 
[148] R. Cutler, D. W. Sivers, Phys. Rev. D17 (1978) 196.

[149] R. Cutler, D. W. Sivers, Phys. Rev. D16 (1977) 679.

[150] B. L. Combridge, J. Kripfganz, J. Ranft, Phys. Lett. B70 (1977) 234.

[151] F. Aversa, P. Chiappetta, M. Greco, J. P. Guillet, Nucl. Phys. B327 (1989) 105.

[152] B. Jager, A. Schafer, M. Stratmann, W. Vogelsang, Phys. Rev. D67 (2003) 054005 .

[153] E. D. Bloom, et al., Phys. Rev. Lett. 23 (1969) 930.

[154] M. Breidenbach, et al., Phys. Rev. Lett. 23 (1969) 935.

[155] J. D. Bjorken, Phys. Rev. 179 (1969) 1547.

[156] M. May, et al., Phys. Rev. Lett. 35 (1975) 407.

[157] M. Arneodo, et al., Nucl. Phys. B481 (1996) 3.

[158] D. Antreasyan, et al., Phys. Rev. D19 (1979) 764.

[159] A. Krzywicki, J. Engels, B. Petersson, U. Sukhatme, Phys. Lett. B85 (1979) 407.

[160] M. Lev, B. Petersson, Z. Phys. C21 (1983) 155.

[161] C. N. Brown, et al., Phys. Rev. C54 (1996) 3195.

[162] A. L. S. Angelis, et al., Phys. Lett. B185 (1987) 213.

[163] M. M. Aggarwal, et al., Eur. Phys. J. C23 (2002) 225.

[164] J. Slivova, Ph.D thesis, Charles University, Prague, 2003, from the CERES experiment. (2003).

[165] R. Albrecht, et al., Eur. Phys. J. C5 (1998) 255.

[166] D. d'Enterria, Phys. Lett. B596 (2004) 32.

[167] H. Busching, J. Phys. G31 (2005) S473.

[168] D. Kharzeev, Y. V. Kovchegov, K. Tuchin, Phys. Rev. D68 (2003) 094013.

[169] H. Baer, J. Ohnemus, J. F. Owens, Phys. Rev. D42 (1990) 61.

[170] P. Aurenche, A. Douiri, R. Baier, M. Fontannaz, D. Schiff, Phys. Lett. B140 (1984) 87.

[171] P. Aurenche, R. Baier, M. Fontannaz, D. Schiff, Nucl. Phys. B297 (1988) 661.

[172] L. E. Gordon, W. Vogelsang, Phys. Rev. D48 (1993) 3136.

[173] J. D. Bjorken, fERMILAB-PUB-82-059-THY (1982). 
[174] M. Gyulassy, M. Plumer, Phys. Lett. B243 (1990) 432.

[175] X.-N. Wang, M. Gyulassy, Phys. Rev. Lett. 68 (1992) 1480.

[176] X.-N. Wang, M. Gyulassy, M. Plumer, Phys. Rev. D51 (1995) 3436.

[177] R. Baier, Y. L. Dokshitzer, S. Peigne, D. Schiff, Phys. Lett. B345 (1995) 277.

[178] R. Baier, Y. L. Dokshitzer, A. H. Mueller, S. Peigne, D. Schiff, Nucl. Phys. B483 (1997) 291.

[179] R. Baier, Y. L. Dokshitzer, A. H. Mueller, D. Schiff, Phys. Rev. C58 (1998) 1706.

[180] M. Gyulassy, P. Levai, I. Vitev, Phys. Rev. Lett. 85 (2000) 5535.

[181] M. Gyulassy, P. Levai, I. Vitev, Nucl. Phys. B594 (2001) 371.

[182] C. Adler, et al., Phys. Rev. Lett. 89 (2002) 202301.

[183] J. Adams, et al., Phys. Rev. Lett. 91 (2003) 172302.

[184] B. B. Back, et al., Phys. Lett. B578 (2004) 297.

[185] X.-N. Wang, Phys. Lett. B595 (2004) 165.

[186] J.-w. Qiu, I. Vitev, Phys. Lett. B570 (2003) 161.

[187] E. Wang, X.-N. Wang, Phys. Rev. Lett. 87 (2001) 142301.

[188] E. Wang, X.-N. Wang, Phys. Rev. Lett. 89 (2002) 162301.

[189] J. W. Cronin, et al., Phys. Rev. D11 (1975) 3105.

[190] D. Antreasyan, et al., Phys. Rev. Lett. 39 (1977) 513.

[191] M. Gyulassy, P. Levai, Phys. Lett. B442 (1998) 1.

[192] X.-N. Wang, Phys. Rev. C61 (2000) 064910.

[193] G. Papp, P. Levai, G. I. Fai, Phys. Rev. C61 (2000) 021902.

[194] I. Vitev, M. Gyulassy, Phys. Rev. Lett. 89 (2002) 252301.

[195] I. Vitev, Phys. Lett. B562 (2003) 36.

[196] X.-N. Wang, Phys. Lett. B565 (2003) 116.

[197] P. Levai, G. Papp, G. G. Barnafoldi, G. I. Fai, nucl-th/0306019 (2003).

[198] D. Kharzeev, E. Levin, L. McLerran, Phys. Lett. B561 (2003) 93.

[199] C. Adler, et al., Phys. Rev. Lett. 90 (2003) 032301.

[200] C. Adler, et al., Phys. Rev. Lett. 90 (2003) 082302.

[201] J. Rak, J. Phys. G30 (2004) S1309. 
[202] M. Chiu, Nucl. Phys. A715 (2003) 761.

[203] S. A. Bass, et al., Nucl. Phys. A661 (1999) 205.

[204] A. Kovner, U. A. Wiedemann, hep-ph/0304151 (2003).

[205] M. Gyulassy, I. Vitev, X.-N. Wang, B.-W. Zhang, nucl-th/0302077 (2003).

[206] M. Gyulassy, M. Plumer, M. Thoma, X. N. Wang, Nucl. Phys. A538 (1992) $37 \mathrm{c}$.

[207] B. G. Zakharov, JETP Lett. 65 (1997) 615.

[208] R. Baier, D. Schiff, B. G. Zakharov, Ann. Rev. Nucl. Part. Sci. 50 (2000) 37.

[209] M. Gyulassy, P. Levai, I. Vitev, Nucl. Phys. B571 (2000) 197.

[210] P. Levai, et al., Nucl. Phys. A698 (2002) 631.

[211] I. Vitev, M. Gyulassy, P. Levai, hep-ph/0109198 (2001).

[212] I. Vitev, J. Phys. G30 (2004) S791.

[213] M. Gyulassy, I. Vitev, X.-N. Wang, P. Huovinen, Phys. Lett. B526 (2002) 301.

[214] X.-f. Guo, X.-N. Wang, Phys. Rev. Lett. 85 (2000) 3591.

[215] A. Airapetian, et al., Eur. Phys. J. C20 (2001) 479.

[216] K. J. Eskola, V. J. Kolhinen, C. A. Salgado, Eur. Phys. J. C9 (1999) 61.

[217] X.-N. Wang, Nucl. Phys. A750 (2005) 98.

[218] K. Gallmeister, C. Greiner, Z. Xu, Phys. Rev. C67 (2003) 044905.

[219] W. Cassing, K. Gallmeister, C. Greiner, Nucl. Phys. A735 (2004) 277.

[220] X.-N. Wang, Phys. Lett. B579 (2004) 299.

[221] M. Gyulassy, P. Levai, I. Vitev, Phys. Lett. B538 (2002) 282.

[222] R. Baier, Y. L. Dokshitzer, A. H. Mueller, D. Schiff, JHEP 0109 (2001) 033.

[223] B. R. Webber, Int. J. Mod. Phys. A15S1 (2000) 577.

[224] B. Alper, et al., Nucl. Phys. B100 (1975) 237.

[225] P. Abreu, et al., Eur. Phys. J. C17 (2000) 207.

[226] I. G. Bearden, et al., Phys. Rev. C66 (2002) 044907.

[227] T. Anticic, et al., Phys. Rev. C69 (2004) 024902.

[228] L. Ahle, et al., Phys. Rev. C55 (1997) 2604.

[229] L. Ahle, et al., Nucl. Phys. A638 (1998) 427.

[230] K. P. Das, R. C. Hwa, Phys. Lett. B68 (1977) 459. 
[231] R. G. Roberts, R. C. Hwa, S. Matsuda, J. Phys. G5 (1979) 1043.

[232] E. Braaten, Y. Jia, T. Mehen, Phys. Rev. Lett. 89 (2002) 122002.

[233] R. J. Fries, B. Muller, C. Nonaka, S. A. Bass, Phys. Rev. Lett. 90 (2003) 202303.

[234] R. J. Fries, B. Muller, C. Nonaka, S. A. Bass, Phys. Rev. C68 (2003) 044902.

[235] R. C. Hwa, C. B. Yang, Phys. Rev. C70 (2004) 024905.

[236] R. C. Hwa, C. B. Yang, Phys. Rev. C67 (2003) 034902.

[237] V. Greco, C. M. Ko, P. Levai, Phys. Rev. Lett. 90 (2003) 202302.

[238] S. A. Voloshin, Nuclear Physics A715 (2003) 379c.

[239] J. Adams, et al., Phys. Rev. Lett. 92 (2004) 052302.

[240] M. Kaneta, J. Phys. G30 (2004) S1217-S1220.

[241] V. Greco, C. M. Ko, Phys. Rev. C70 (2004) 024901.

[242] X. Dong, S. Esumi, P. Sorensen, N. Xu, Z. Xu, Phys. Lett. B597 (2004) 328.

[243] PHENIX Collaboration, BNL 72162-2004, internal report (2003).

[244] T. Matsui, H. Satz, Phys. Lett. B178 (1986) 416.

[245] M. C. Abreu, et al., Phys. Lett. B477 (2000) 28.

[246] A. Capella, E. G. Ferreiro, A. B. Kaidalov, Phys. Rev. Lett. 85 (2000) 2080.

[247] A. Capella, A. B. Kaidalov, D. Sousa, Phys. Rev. C65 (2002) 054908.

[248] R. L. Thews, M. Schroedter, J. Rafelski, Phys. Rev. C63 (2001) 054905.

[249] R. L. Thews, J. Phys. G30 (2004) S369.

[250] A. P. Kostyuk, M. I. Gorenstein, H. Stocker, W. Greiner, Phys. Rev. C68 (2003) 041902.

[251] P. Braun-Munzinger, J. Stachel, Phys. Lett. B490 (2000) 196.

[252] A. Andronic, P. Braun-Munzinger, K. Redlich, J. Stachel, Phys. Lett. B571 (2003) 36 .

[253] M. G. Mustafa, D. Pal, D. K. Srivastava, M. Thoma, Phys. Lett. B428 (1998) 234.

[254] Z.-w. Lin, R. Vogt, X.-N. Wang, Phys. Rev. C57 (1998) 899.

[255] Y. L. Dokshitzer, D. E. Kharzeev, Phys. Lett. B519 (2001) 199.

[256] G. Agakishiev, et al., Phys. Rev. Lett. 75 (1995) 1272.

[257] G. Agakishiev, et al., Phys. Lett. B422 (1998) 405. 
[258] D. Adamova, et al., Phys. Rev. Lett. 91 (2003) 042301.

[259] R. Rapp, J. Wambach, Adv. Nucl. Phys. 25 (2000) 1.

[260] R. Arnaldi, et al., nucl-ex/0501028 (2005).

[261] R. Rapp, nucl-th/0204003 (2002).

[262] Z. Fraenkel, B. Khachaturov, A. Kozlov, A. Milov, D. Mukhopadhyay, D. Pal, I. Ravinovich, I. Tserruya, S. Zhou, PHENIX Technical Note 391, internal report (2001).

[263] A. Kozlov, et al., Nucl. Instrum. Meth. A523 (2004) 345.

[264] R. Rapp, Phys. Rev. C63 (2001) 054907.

[265] P. V. Ruuskanen, Nucl. Phys. A544 (1992) 169.

[266] G.-Q. Li, C. Gale, Phys. Rev. Lett. 81 (1998) 1572.

[267] S. Turbide, R. Rapp, C. Gale, Phys. Rev. C69 (2004) 014903. 